2. To: (Receiving Organization)

Waste Feed Delivery

5. Proj./Prog./Dept./Div.:

RPP

8. Originator Remarks:

Final report on tank $241-\mathrm{C}-104$ retrieval studies.

11. Receiver Remarks: ................... 11A. Design Baseline Document? $\bigcirc$ Yes $\bigcirc$ No

3. From: (Originating Organization)
TOPS
$\begin{aligned} & \text { 6. Design Authority/Design Agent/Cog. Engr.: } \\ & \text { J. F. O'Rourke }\end{aligned}$

3. From: (Originating Organization)

4. Related EDT No::

$\mathrm{N} / \mathrm{A}$

7. Purchase Order No.:

$\mathrm{N} / \mathrm{A}$

9. Equip./Component No.:

$\mathrm{N} / \mathrm{A}$

10. System/Bidg./Facility:

222-S Laboratory *

12. Major Assm. Dwg. No.:

$\mathrm{N} / \mathrm{A}$

13. Permit/Permit Application No::

$\mathrm{N} / \mathrm{A}$

14. Required Response Date:

February 3, 2000

15.

\begin{tabular}{|l|l|}
\hline $\begin{array}{c}\text { (A) } \\
\text { lfem } \\
\text { No. }\end{array}$ & (B) Document/Drawing No. \\
\hline 1 & RPP -5798 \\
\hline & \\
\hline & \\
\hline & \\
\hline & \\
\hline & \\
\hline
\end{tabular}

DATA TRANSMITTED

$\begin{array}{lll}\text { (C) Sheet } & \text { (D) Rev. } & \text { (E) Title or Description of Data Transmitted }\end{array}$
(F)

(G)

\begin{tabular}{|c|c|c|c|}
$\begin{array}{c}\text { Approval } \\
\text { Desig- } \\
\text { nator }\end{array}$ & $\begin{array}{c}\text { Reason } \\
\text { for Trans- } \\
\text { mittal }\end{array}$ & $\begin{array}{c}\text { Origi- } \\
\text { nator } \\
\text { Dispo- } \\
\text { stion }\end{array}$ & $\begin{array}{c}\text { Receiv- } \\
\text { er } \\
\text { Dispo- } \\
\text { sition }\end{array}$ \\
\hline N/A & 1 & 1 &
\end{tabular}

16.

\begin{tabular}{|c|c|}
\hline Approval Designator $(F)$ & \\
\hline E, S, Q, D OR N/A & 1. Approval \\
(See WHC-CM-3-5, & 2. Release \\
Sec. 12.7) & 3. Information \\
\hline
\end{tabular}

17.

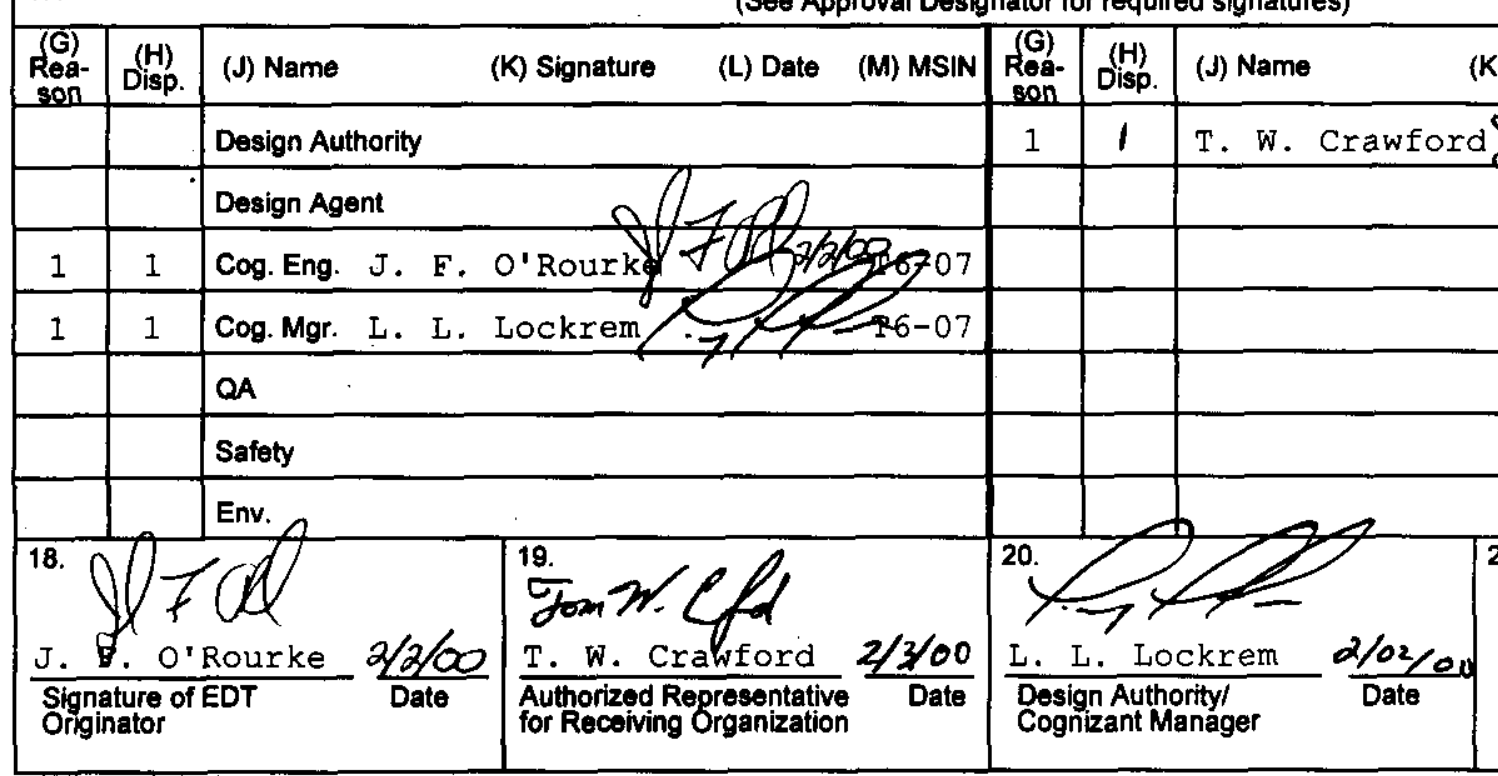

Disposition (H) \& (l)

Reason for Transmittal (G)

4. Review

6. Pist. (Receipt Acknow. Required)

1. Approved

3. Disapproved w/comment

4. Reviewed no/comment 5. Reviewed no/comment 6. Receipt acknowledged

SIGNATUREIDISTRIBUTION

SIGNATURE/DISTRIBUTION
(See Approval Designator for required signatures)
(K) Signature

(L) Date

(M) MSIN
21. DOE APPROVAL (if required) Ctrl No.

Approved

Approved w/comments

- Disapproved w/comments 


\title{
Results of Retrieval Studies with Waste from Tank 241-C-104
}

\author{
J. F. O'Rourke \\ Eluor Hanford \\ Richland, WA 99352 \\ U.S. Department of Energy Contract DE-AC06-96RL13200

$\begin{array}{lll}\text { EDT/ECN: } & 620367 & \text { UC: } 2030 \\ \text { Org Code: } & 8 \mathrm{D} 500 & \text { Charge Code: } 102199 \\ \text { B\&R Code: } & \text { EW3130010 } & \text { Total Pages: } 147\end{array}$

Key Words: retrieval, target dilution, technetium, viscosity, density, shear strength, particle size distribution, settling, slurry

Abstract: Laboratory studies were performed on samples of waste from Tank 241-C-104. Physical property data was gathered to develop engineering plans for retrieval operations. Chemical composition data was collected to verify the ability to meet contract feed specifications.

* Haake is a registered trademark of Haake Medingen, GmbH. * Horiba is a registered trademark of Horiba, Ltd.

TRADEMARK DISCLAIMER. Reference herein to any specific commercial product, process, or service by trade name, trademark, manufacturer, or otherwise, does not necessarily constitute or imply its endorsement, recommendation, or favoring by the United States Government or any agency thereof or its contractors or subcontractors.

Printed in the United States of Americe. To obtain copies of this document, contact: Document Control Services, P.O. Box 950, Mailstop H6-08, Richland WA 99352, Phone (509) 372-2420; Fax (509) 376-4989.
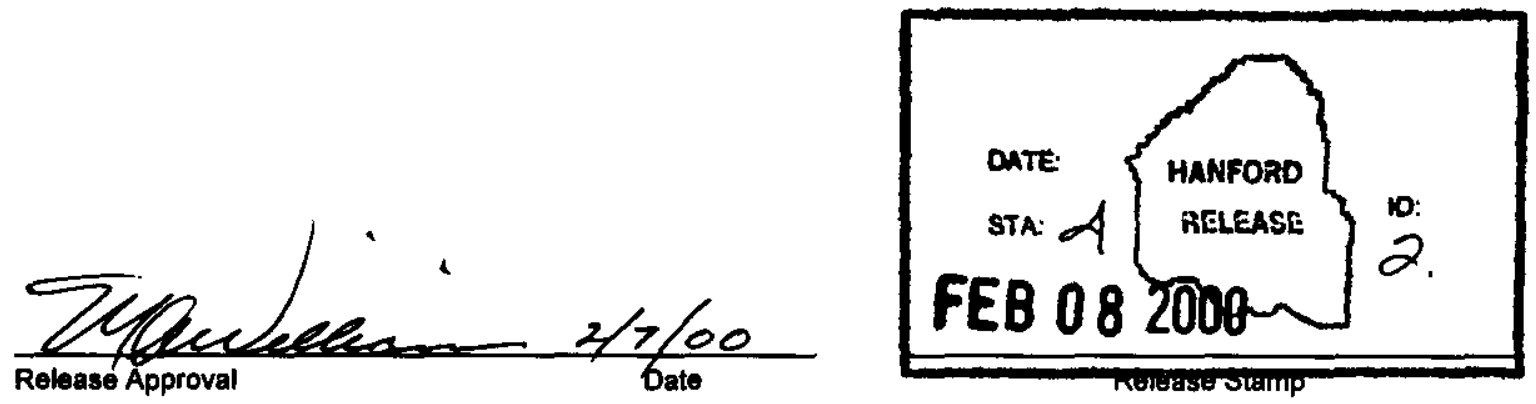


\section{CONTENTS}

1.0 INTRODUCTION AND EXECUTIVE SUMMARY............................................ 1

1.1 SUMMARY AND CONCLUSIONS ............................................................... 1

2.0 DESCRIPTION OF LABORATORY STUDIES ..................................................... 3

2.1 PREPARATION OF WHOLE-TANK-COMPOSITE SAMPLES ........................ 3

2.2 PREPARATION OF DILUTED SAMPLES......................................................... 4

2.2.1 Preparation of Target Dilution Samples ......................................................... 4

2.2.2 Preparation of $60 \mathrm{~g} / \mathrm{L}$ and $140 \mathrm{~g} / \mathrm{L}$ Samples ............................................ 7

2.2.3 Preparation of $200 \mathrm{~g} / \mathrm{L}$ and $300 \mathrm{~g} / \mathrm{L}$ Samples ......................................... 7

2.3 DENSITY AND SETTLING RATE MEASUREMENTS.................................... 7

2.4 PHYSICAL PROPERTIES MEASUREMENTS .................................................. 8

2.5 SLUDGE WASHING AND CAUSTIC LEACHING........................................ 9

2.6 SLUDGE COMPOSITION AS A FUNCTION OF SETTLING ........................... 9

3.0 RESULTS OF ANALYTICAL MEASUREMENTS.............................................10

3.1 WHOLE-TANK-COMPOSITE TECHNETIUM-99 ANALYSIS.........................10

3.2 PHYSICAL PROPERTY MEASUREMENTS ................................................ 10

3.2.1 Shear Strength Results .......................................................................... 10

3.2.2 Viscosity Results............................................................................................. 11

3.2.3 Particle Size Distribution Results .................................................................13

3.3 DENSITY AND SETTLING RATE MEASUREMENTS..................................... 14

3.3.1 Density Measurements.............................................................................. 14

3.3.2 Bulk Density of Undiluted Sludge ................................................................. 15

3.3.3 Settling Rate Measurements ....................................................................... 16

3.4 TARGET DILUTION CHEMICAL COMPOSITION ............................................ 16

3.5 SLUDGE COMPOSITION AS A FUNCTION OF SETTLING ...........................26

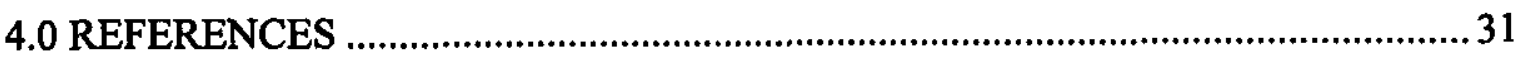

APPENDIX A, Viscosity Data.....................................................................................

APPENDIX B, Particle Size Distribution Data ............................................................... B-1 
RPP-5798, Rev. 0

\section{TABLES}

2-1 Tank C-104 Core Samples Used in WTC Samples .................................................. 3

2-2 Liquid Phase Analyses for Target Dilution Samples................................................ 4

2-3 Centrifuged Solids Analyses for Target Dilution Samples ........................................ 5

3-1 Technetium-99 Analysis of WTC Sample.................................................................1 10

3-2 Results of Shear Strength Measurements .................................................................... 11

3-3 Lower Operating Range of Haake M5 Viscometer ....................................................... 11

3-4 Summary of Viscosity Measurements.........................................................................12

3-5 Summary of Particle Size Distribution Measurements.................................................. 14

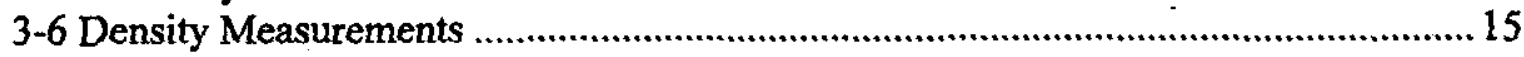

3-7 Slurry-Liquid Interface Levels ...............................................................................17

3-8 Multiplication Factors for Mass Balance Calculation .................................................... 18

3-9 Composition of Target Dilution Supernatant Liquid..................................................2

3-10 Composition of Target Dilution Centrifuged Solids ....................................................22

3-11 Comparison of Fusion and Acid Digest for ICP/AES Analyses ................................25

3-12 Sludge Composition as a Function of Sludge Settling ...............................................2 
RPP-5798, Rev. 0

\section{FIGURES}

3-1 Viscosity as a Function of Solids Concentration.......................................................13

3-2 Settling Study with Diluted Sludge ....................................................................... 16

3-3 Normalized Concentrations of Selected Species as a Function of Settling ................29

3-4 Normalized Concentrations of Selected Species as a Function of Settling (Duplicate Sample) ....................................................................................................29

3-5 Normalized Radiochemical Concentrations as a Function of Settling ..........................30

3-6 Normalized Radiochemical Concentrations as a Function of Settling (Duplicate Sample). 
RPP-5798, Rev. 0

\section{ABBREVIATIONS, ACRONYMS, AND SYMBOLS}

C-104 Tank 241-C-104

AEA alpha energy analysis

AT/TB total alpha/total beta activity

cP centiPoise

ESP Environmental Simulation Program

g gram

GEA gamma energy analysis

$\mathrm{g} / \mathrm{mL} \quad$ gram per milliliter

IC ion chromatography

ICP/AES inductively coupled plasma atomic emission spectroscopy

ICP/MS inductively coupled plasma mass spectroscopy

in. inch

IW inhibited water

$\min$ minute

$\mathrm{mL} \quad$ milliliter

mPas milliPascal-second (equivalent to centiPoise)

$\mathrm{Pa} \quad$ Pascal

RPP River Protection Project

$\mathrm{s}^{-1} \quad$ reciprocal second

$t$ time

Temp temperature

TD target dilution ( $100 \mathrm{~g} / \mathrm{L}$ solids / $\mathrm{L}$ diluted sludge)

TGA thermogravimetric analysis

TIC total inorganic carbon

TOC total organic carbon

WTC whole-tank-composite

$\gamma \quad$ shear rate .

$\eta \quad$ viscosity

$\tau \quad$ shear stress 


\subsection{INTRODUCTION AND EXECUTIVE SUMMARY}

Tank 241-C-104 (C-104) has been selected as a Phase I source tank for High Level Waste sludge feed for vitrification. Rheological data are needed to develop engineering plans for the retrieval operations. Chemical composition data are needed to verify the ability to meet contract feed specifications. Other physical data are needed to support computational fluid dynamics modeling of sludge mobilization. The requirements for this study are discussed in Data Quality Objectives for TWRS Privatization Phase 1: Confirm Tank T is an Appropriate Feed Source for High-Level Waste Feed Batch X (Nguyen 1999a). The tests were carried out according to a prescribed test plan (Herting et al. 1999).

Shear strength measurements were made on twelve undisturbed portions of core-sampled C-104 sludge. Aliquots from these samples were later withdrawn to prepare the wholetank-composite (WTC) samples used in the remainder of the study.

A "target dilution" was assigned for the transport of C-104 sludge to tank AY-101, such that the diluted sludge will contain 100 grams of solids per liter of diluted sludge $(100 \mathrm{~g} / \mathrm{L})$. Physical measurements (density, settling rate, viscosity, particle size distribution) were performed on the sludge at the target dilution and at one dilution level on either side of the target $(60 \mathrm{~g} / \mathrm{L}$ and $140 \mathrm{~g} / \mathrm{L})$. Viscosity measurements were also made on the undiluted WTC and two samples with a higher solids concentration $(200 \mathrm{~g} / \mathrm{L}$ and $300 \mathrm{~g} / \mathrm{L}$ ).

Aliquots from both the liquid and solid phases of the target dilution were submitted for extensive chemical and radiological analyses. A separate test examined the composition of the settled solids in the target dilution as a function of settling rate.

\subsection{SUMMARY AND CONCLUSIONS}

A "target dilution" was assigned such that the diluted sludge contained 100 grams of solids per liter of diluted sludge $(100 \mathrm{~g} / \mathrm{L})$. Most of the physical property and chemical composition measurements were performed on samples at the target dilution. In addition, samples were also prepared at one dilution level on either side of the target $(60 \mathrm{~g} / \mathrm{L}$ and $140 \mathrm{~g} / \mathrm{L}$ ). Several physical properties measurements were made at the $60 \mathrm{~g} / \mathrm{L}$ and $140 \mathrm{~g} / \mathrm{L}$ dilutions, including density, settling rate, viscosity, and particle size distribution.

Before starting any other tests, shear strength measurements were completed on the undisturbed sludge. The shear strengths ranged from 31 to 760 Pascals and tended to increase with sample depth in the tank. The shear strength could not be measured for the two samples collected from the lowest segment. These two samples were sufficiently hard that the shear vane could not be pushed into the sample material. 
The viscosity was measured on sludge at the five dilution levels, whole tank composite, and decanted supernatant liquid from the target dilution sample. Each of the viscosity measurements was performed at ambient, $45^{\circ} \mathrm{C}$, and $65^{\circ} \mathrm{C}$. The viscosity of the diluted samples increased dramatically between the $200 \mathrm{~g} / \mathrm{L}$ and $300 \mathrm{~g} / \mathrm{L}$ dilution levels.

For the settling study, the position of the slurry-liquid interface was measured for the three dilution levels over the duration of one week. The three dilution conditions had the characteristic settling shape of rapid, unhindered settling followed by a longer period of compaction. The rapid settling phase was completed for the three dilution levels within the first twenty-four hours. The three dilution conditions reached their final solids volume after three days of settling.

Duplicate samples of the target dilution were centrifuged prior to submitting the solid and liquid fractions for sample analysis. No separable organic phase was apparent after 30 minutes of centrifugation.

Duplicate samples of the target dilution were allowed to settle and segregated into three fractions based upon their location after settling. Samples of each fraction were submitted for analysis. The aluminum concentrations were greater in the samples collected from the middle third fractions. Most of the other species had a corresponding decrease in their concentrations in the middle third fractions. The bottom third samples had alpha and beta levels significantly higher than the other two fractions. 


\subsection{DESCRIPTION OF LABORATORY STUDIES}

\subsection{PREPARATION OF WHOLE-TANK-COMPOSITE SAMPLES}

The WTC samples were prepared in a hot cell. The WTC samples were prepared as directed in the test procedure (Beck 1999). Shear strength measurements were made on each of the undisturbed samples prior to the WTC sample preparation. The shear strength measurements are discussed in more detail in Sections 2.4 and 3.2.1.

To the extent possible, the WTC samples were prepared as specified in the test procedure. The contents of each segment jar were thoroughly mixed and then transferred into two $500 \mathrm{~mL}$ composite jars. Approximately $44 \mathrm{~g}$ per half segment and $22 \mathrm{~g}$ per quarter segment were placed in each of the two composite jars (Beck 1999). Table 2-1 shows a summary of the sample jars used to prepare the two WTC samples. The WTC samples were thoroughly mixed after the final addition.

Table 2-1 Tank C-104 Core Samples Used in WTC Samples

\begin{tabular}{|c|c|c|c|c|}
\hline Core & Segment & $\begin{array}{c}\text { Jar } \\
\text { Number }\end{array}$ & $\begin{array}{c}\text { Comp \#1 } \\
\text { Weight Used } \\
\text { (grams) }\end{array}$ & $\begin{array}{c}\text { Comp \#2 } \\
\text { Weight Used } \\
\text { (grams) }\end{array}$ \\
\hline 247 & 1 Upper Solids & 13596 & 44.5 & 46.1 \\
\hline 247 & 1 Lower Solids & 13607 & 44.5 & 43.8 \\
\hline 247 & 2 Upper Solids & 14737 & 44.4 & 46.0 \\
\hline 247 & 2 Lower Solids & 14754 & 42.7 & 44.9 \\
\hline 247 & 3 Quarter A & 13017 & 23.0 & 22.0 \\
\hline 247 & 3 Quarter B & 13018 & 22.6 & 22.5 \\
\hline 247 & 3 Quarter C & 13019 & 24.8 & 25.1 \\
\hline 247 & 3 Quarter D & 13020 & 22.4 & 22.8 \\
\hline 247 & 4 Upper Solids & 12396 & 45.4 & 45.8 \\
\hline 247 & 4 Lower Solids & 12395 & 44.4 & 44.2 \\
\hline 247 & 5 Upper Solids & 13012 & 43.2 & 45.0 \\
\hline 247 & 5 Upper Solids & 13013 & 55.1 & 49.5 \\
\hline
\end{tabular}

The WTC samples were used for the preparation of the target dilution and other dilution level samples. In addition, some analyses were performed on the undiluted WTC samples. These analyses included ${ }^{99} \mathrm{Tc}$ (both ICP/MS and liquid scintillation), viscosity, and particle size distribution. 


\subsection{PREPARATION OF DILUTED SAMPLES}

\subsubsection{Preparation of Target Dilution Samples}

Retrieval of the sludge from tank $\mathrm{C}-104$ will require dilution to transport the sludge to tank AY-101. The "target dilution" has been assigned such that the diluted sludge will contain 100 grams of solids per liter of diluted sludge $(100 \mathrm{~g} / \mathrm{L})$. The target dilution samples were measured for several physical properties, including density, settling rate, viscosity, particle size distribution, and composition of settled solids as a function of settling time. In addition, duplicate samples of sludge at the target dilution were separated by centrifugation at ambient temperature into supernatant liquid and centrifuged solids fractions. Supernatant liquid samples were analyzed for the "envelopes A, B, C" analytes shown in Table 2-2 (Nguyen 1999b). The solids were analyzed according to the "envelope D" list shown in Table 2-3 (Nguyen 1999a).

Several analyses (Pd, Pr, Rb, Rh, Ru, Ta, Te, W, Y, ${ }^{\text {total }} \mathrm{Cs}$, and ${ }^{238} \mathrm{Pu}$ ) included in Tables 2-2 and 2-3 were not performed due to the lack of adequate methods at the 222-S Laboratory. These analytes are of limited interest for waste retrieval, but will become increasingly important for melter operations.

Table 2-2 Liquid Phase Analyses for Target Dilution Samples

\begin{tabular}{|c|c|c|}
\hline Procedure & Method & Analytes \\
\hline$\overline{L A-505-161}$ & ICP/AES & $\begin{array}{l}\mathrm{Ag}, \mathrm{Al}, \mathrm{As}, \mathrm{B}, \mathrm{Ba}, \mathrm{Be}, \mathrm{Bi}, \mathrm{Ca}, \mathrm{Cd}, \mathrm{Ce}, \mathrm{Co}, \mathrm{Cr} \text {, } \\
\mathrm{Cu}, \mathrm{Fe}, \mathrm{K}, \mathrm{La}, \mathrm{Li}, \mathrm{Mg}, \mathrm{Mn}, \mathrm{Mo}, \mathrm{Na}, \mathrm{Nd}, \mathrm{Ni} \\
\mathrm{P}, \mathrm{Pb}, \mathrm{Pd}, \mathrm{Pr}, \mathrm{Rb}, \mathrm{Rh}, \mathrm{Ru}, \mathrm{S}, \mathrm{Sb}, \mathrm{Se}, \mathrm{Si}, \mathrm{Sm} \\
\mathrm{Sr}, \mathrm{Ta}, \mathrm{Te}, \mathrm{Th}, \mathrm{Ti}, \mathrm{Tl}, \mathrm{U}, \mathrm{V}, \mathrm{W}, \mathrm{Y}, \mathrm{Zn}, \mathrm{Zr}\end{array}$ \\
\hline LA-533-105 & $\overline{\mathrm{IC}}$ & $\mathrm{Br}, \mathrm{Cl}^{-}, \mathrm{F}^{-}, \mathrm{NO}_{2}^{-}, \mathrm{NO}_{3}^{-}, \mathrm{C}_{2} \mathrm{O}_{4}^{2-}, \mathrm{PO}_{4}^{3-}, \mathrm{SO}_{4}^{2-}$ \\
\hline LA-510-112 & Specific Gravity & density \\
\hline LA-325-104 & CVAA & $\mathrm{Hg}$ \\
\hline LA-342-100 & Persulfate/Combustion & TIC/TOC \\
\hline LA-212-106 & $\mathrm{pH}$ & $\mathrm{pH}$ \\
\hline LA-514-114 & TGA & $\% \mathrm{H}_{2} \mathrm{O}$ \\
\hline LA-211-102 & Titration & $\mathrm{OH}^{-}$ \\
\hline LA-506-101 & ICP/MS & $\begin{array}{c}{ }^{99} \mathrm{Tc},{ }^{126} \mathrm{Sn},{ }^{\text {Total }} \mathrm{Cs},{ }^{233 / 239 / 2435} \mathrm{U},{ }^{237} \mathrm{~Np} \\
{ }^{23 / 42} \mathrm{Pu},{ }^{243} \mathrm{Am}\end{array}$ \\
\hline LA-438-101 & Liquid Scintillation & ${ }^{99} \mathrm{Tc}$ \\
\hline LA-548-121 & GEA & ${ }^{60} \mathrm{Co},{ }^{125} \mathrm{Sb},{ }^{137} \mathrm{Cs},{ }^{152 / 154 / 155} \mathrm{Eu}$ \\
\hline LA-220-101 & Separation \& Beta Counting & ${ }^{90} \mathrm{Sr}$ \\
\hline LA-508-101 & Alpha/Beta Counting & total alpha/total beta \\
\hline
\end{tabular}


RPP-5798, Rev. 0

Table 2-3 Centrifuged Solids Analyses for Target Dilution Samples

\begin{tabular}{|c|c|c|c|}
\hline Procedure & Method & Prep & Analytes \\
\hline LA-505-161 & ICP/AES & $\begin{array}{c}\text { Acid } \\
\text { Digest }\end{array}$ & $\begin{array}{l}\mathrm{Ag}, \mathrm{Al}, \mathrm{As}, \mathrm{B}, \mathrm{Ba}, \mathrm{Be}, \mathrm{Bi}, \mathrm{Ca}, \mathrm{Cd}, \mathrm{Ce}, \mathrm{Co}, \mathrm{Cr} \\
\mathrm{Cu}, \mathrm{Fe}, \mathrm{K}, \mathrm{La}, \mathrm{Li}, \mathrm{Mg}, \mathrm{Mn}, \mathrm{Mo}, \mathrm{Na}, \mathrm{Nd}, \mathrm{Ni} \\
\mathrm{P}, \mathrm{Pb}, \mathrm{Pd}, \mathrm{Pr}, \mathrm{Rb}, \mathrm{Rh}, \mathrm{Ru}, \mathrm{S}, \mathrm{Sb}, \mathrm{Se}, \mathrm{Si}, \mathrm{Sm} \\
\mathrm{Sr}, \mathrm{Ta}, \mathrm{Te}, \mathrm{Th}, \mathrm{Ti}, \mathrm{Tl}, \mathrm{U}, \mathrm{V}, \mathrm{W}, \mathrm{Y}, \mathrm{Zn}, \mathrm{Zr}\end{array}$ \\
\hline LA-505-161 & ICP/AES & Fusion & Same as Above \\
\hline LA-533-105 & IC & $\begin{array}{l}\text { Water } \\
\text { Digest }\end{array}$ & $\mathrm{Br}^{-}, \mathrm{Cl}^{-}, \mathrm{F}^{-}, \mathrm{NO}_{2}^{-}, \mathrm{NO}_{3}^{-}, \mathrm{C}_{2} \mathrm{O}_{4}^{2-}, \mathrm{PO}_{4}^{3-}, \mathrm{SO}_{4}^{2-}$ \\
\hline LA-325-104 & CVAA & Direct & $\mathrm{Hg}$ \\
\hline LA-342-100 & $\begin{array}{l}\text { Persulfate/ } \\
\text { Combustion }\end{array}$ & Direct & TIC/TOC \\
\hline LA-514-114 & TGA & Direct & $\% \mathrm{H}_{2} \mathrm{O}$ \\
\hline LA-695-102 & Colorimetry & Direct & $\mathrm{CN}^{-}$ \\
\hline LA-211-102 & Titration & $\begin{array}{l}\text { Water } \\
\text { Digest }\end{array}$ & $\mathrm{OH}^{-}$ \\
\hline LA-631-001 & $\begin{array}{l}\text { Ion Selective } \\
\text { Electrode }\end{array}$ & $\begin{array}{l}\text { Water } \\
\text { Digest }\end{array}$ & $\mathrm{NH}_{3}$ \\
\hline LA-506-101 & ICP/MS & Fusion & $\begin{array}{c}{ }^{99} \mathrm{Tc},{ }^{126} \mathrm{Sn},{ }^{\text {Total }} \mathrm{Cs},{ }^{233 / 239 / 240 / 241 / 242} \mathrm{Pu},{ }^{243} \mathrm{Um} \\
\end{array}$ \\
\hline LA-953-104 & Separation/AEA & Fusion & ${ }^{243} \mathrm{Am},{ }^{243 / 244} \mathrm{Cm}$ \\
\hline LA-438-101 & $\begin{array}{c}\text { Liquid } \\
\text { Scintillation } \\
\end{array}$ & Fusion & ${ }^{99} \mathrm{Tc}$ \\
\hline LA-548-121 & GEA & Fusion & ${ }^{60} \mathrm{Co},{ }^{125} \mathrm{Sb},{ }^{137} \mathrm{Cs},{ }^{152 / 154 / 155} \mathrm{Eu}$ \\
\hline$\overline{L A-218-114}$ & $\begin{array}{c}\text { Liquid } \\
\text { Scintillation }\end{array}$ & Fusion & ${ }^{3} \mathrm{H}$ \\
\hline LA-348-104 & $\begin{array}{c}\text { Liquid } \\
\text { Scintillation }\end{array}$ & Fusion & ${ }^{14} \mathrm{C}$ \\
\hline LA-220-101 & $\begin{array}{c}\text { Separation \& } \\
\text { Beta Counting }\end{array}$ & Fusion & ${ }^{90} \mathrm{Sr}$ \\
\hline LA-378-103 & Separation/GEA & Fusion & ${ }^{129} \mathrm{I}$ \\
\hline LA-508-101 & $\begin{array}{l}\text { Alpha/Beta } \\
\text { Counting }\end{array}$ & Fusion & total alpha/total beta \\
\hline
\end{tabular}

For purposes of preparing the sludge dilutions $(60,100,140 \mathrm{~g}$ solids per liter of diluted sludge), the percent water in the WTC was assumed to be $50.8 \%$. This was the mean value found previously for the solid segments (Baldwin 1997, Table B3-5). Similarly, a mean value of $80.8 \%$ water was found for the liquid. 
Then, $100 \mathrm{~g}$ of composite would contain:

$$
\begin{gathered}
100 \mathrm{~g} \times \frac{0.508 \mathrm{~g} \mathrm{H}_{2} \mathrm{O}}{\mathrm{g} \text { comp }} \times \frac{\mathrm{g} \text { Liquid }}{0.808 \mathrm{~g} \mathrm{H}_{2} \mathrm{O}}=62.9 \mathrm{~g} \text { Liquid } \\
100 \mathrm{~g} \text { comp }-62.9 \mathrm{~g} \text { Liquid }=37.1 \mathrm{~g} \text { Solids }
\end{gathered}
$$

At a composite density of $1.67 \mathrm{~g} / \mathrm{mL}$ (Baldwin 1997, Table B3-5), this corresponds to $0.620 \mathrm{~g}$ solids per $\mathrm{mL}$ composite, or $620 \mathrm{~g} / \mathrm{L}$.

A computer modeling program known as the Environmental Simulation Program (ESP), produced by OLI Systems, Inc. of Morris Plains, New Jersey, was used to predict the solubilities for the components of the C-104 sludge: The ESP predicted the sludge would contain $36.3 \mathrm{~g}$ solids per $100 \mathrm{~g}$ sludge. This is in reasonable agreement with the above calculation ( $37.1 \mathrm{~g}$ solids / $100 \mathrm{~g}$ sludge). The ESP run predicts the following solids (in decreasing order of abundance): $\mathrm{Al}(\mathrm{OH})_{3}, \mathrm{NaF}, \mathrm{ZrO}_{2}, \mathrm{FeOOH}, \mathrm{Na}_{2} \mathrm{C}_{2} \mathrm{O}_{4}, \mathrm{NaAlSiO}_{4}$, $\mathrm{Mn}(\mathrm{OH})_{2}, \mathrm{Na}_{2} \mathrm{U}_{2} \mathrm{O}_{7}$, and several minor components. These species are all insoluble in inhibited water except for $\mathrm{NaF}$. According to the ESP run, $\mathrm{NaF}$ represents $11.3 \%$ by weight of the solids.

If no solid dissolves, $269.5 \mathrm{~g}$ composite per liter of diluted sludge would be required to produce a $100 \mathrm{~g} / \mathrm{L}$ slurry. If the ESP calculation is correct, the $269.5 \mathrm{~g}$ composite would contain $11.3 \mathrm{~g}$ solid $\mathrm{NaF}$. Assuming almost all of the $\mathrm{NaF}$ dissolves, approximately $10 \%$ of the solids in the composite sample will dissolve upon dilution. Thus, the amount of composite needs to be increased to $296.5 \mathrm{~g}$ to get the desired amount of solids after the dilution. This can be rounded off to $300 \mathrm{~g}$ composite, given the number of significant assumptions (Beck 1999).

The assumption that all of the $\mathrm{NaF}$ would dissolve somewhat overstated the case. The analysis of the target dilution samples revealed that the supernatant liquid contained only $60 \%$ of the available fluoride. This difference is negligible given the number of other assumptions made during the target dilution preparation.

Therefore, $135 \mathrm{~g}$ composite sample was needed with the addition of inhibited water to reach a total volume of 0.45 liter. Assuming the volumes are additive, $135 \mathrm{~g}$ composite $(80 \mathrm{~mL})$ would be mixed with $370 \mathrm{~mL}$ water. During the preparation of the target dilution sample, $135.3 \mathrm{~g}$ composite was mixed with $370.1 \mathrm{~g}$ inhibited water.

Inhibited water $\left(0.01 \mathrm{M} \mathrm{NaOH}\right.$ and $0.01 \mathrm{M} \mathrm{NaNO}_{2}$ in water) was used as a diluent for all dilution levels in this study. Inhibited water is used in tank farms to ensure the operational specifications for corrosion are met. 


\subsubsection{Preparation of $60 \mathrm{~g} / \mathrm{L}$ and $140 \mathrm{~g} / \mathrm{L}$ Samples}

In addition to the target dilution, samples were also prepared at one dilution level on either side of the target $(60 \mathrm{~g} / \mathrm{L}$ and $140 \mathrm{~g} / \mathrm{L})$. These dilution samples had several physical properties measured (density, settling rate, viscosity, particle size distribution).

For the $60 \mathrm{~g} / \mathrm{L}$ and $140 \mathrm{~g} / \mathrm{L}$ dilutions, the assumption was again made that $10 \%$ of the solids in the composite sample would dissolve upon dilution. The preparation recipe was calculated in a similar fashion as the $100 \mathrm{~g} / \mathrm{L}$ dilution sample. For $100 \mathrm{~mL}$ of $60 \mathrm{~g} / \mathrm{L}$ slurry, $18 \mathrm{~g}(11 \mathrm{~mL})$ composite would be mixed with $89 \mathrm{~mL}$ water. For $100 \mathrm{~mL}$ of $140 \mathrm{~g} / \mathrm{L}$ slurry, $41.5 \mathrm{~g}(25 \mathrm{~mL})$ composite would be mixed with $75 \mathrm{~mL}$ water (Beck 1999).

During the preparation of the $60 \mathrm{~g} / \mathrm{L}$ slurry sample, $18.0 \mathrm{~g}$ composite was mixed with $92.3 \mathrm{~g}$ inhibited water. For the $140 \mathrm{~g} / \mathrm{L}$ sample, $41.8 \mathrm{~g}$ composite was mixed with $75.3 \mathrm{~g}$ inhibited water.

\subsubsection{Preparation of $200 \mathrm{~g} / \mathrm{L}$ and $300 \mathrm{~g} / \mathrm{L}$ Samples}

Two dilution samples with higher solids content $(200 \mathrm{~g} / \mathrm{L}$ and $300 \mathrm{~g} / \mathrm{L})$ were added to the study in an effort to determine whether the viscosity increases rapidly with partial settling (Crawford 1999). These samples were evaluated for viscosity only.

As with the other dilution samples, the assumption was made that $10 \%$ of the solids in the composite sample would dissolve upon dilution. The preparation recipe was calculated in a similar fashion as the other dilution samples. For $50 \mathrm{~mL}$ of $200 \mathrm{~g} / \mathrm{L}$ slurry, $30 \mathrm{~g}$ $(18 \mathrm{~mL}$ ) composite would be mixed with $32 \mathrm{~mL}$ water. For $50 \mathrm{~mL}$ of $300 \mathrm{~g} / \mathrm{L}$ slurry, $45 \mathrm{~g}(27 \mathrm{~mL})$ composite would be mixed with $23 \mathrm{~mL}$ water.

During the preparation of the $200 \mathrm{~g} / \mathrm{L}$ slurry sample, $33.2 \mathrm{~g}$ composite was mixed with $30.4 \mathrm{~g}$ inhibited water. For the $300 \mathrm{~g} / \mathrm{L}$ sample, $47.9 \mathrm{~g}$ composite was mixed with $24.2 \mathrm{~g}$ inhibited water.

\subsection{DENSITY AND SETTLING RATE MEASUREMENTS}

Density measurements were determined for the bulk sample, liquid phase, and settled solids for each of the three dilution levels $(60 \mathrm{~g} / \mathrm{L}, 100 \mathrm{~g} / \mathrm{L}$, and $140 \mathrm{~g} / \mathrm{L})$ of the composite sample. The density measurements were derived from samples used for settling rate tests. 
For each settling rate test, approximately $100 \mathrm{~mL}$ of diluted sludge was poured into a tared $100 \mathrm{~mL}$ graduated cylinder and allowed to settle for one week. The position of the interface between the settled solids and supernatant liquid was recorded hourly at the beginning and several times a day near the end of the test. The measurements were recorded by a video camera with time-lapse capabilities. As a backup, a scientific technician on day shift took readings several times throughout the test. At the conclusion of the settling rate test, samples of supernatant liquid from each of the three dilution levels were analyzed for density by procedure LA-510-112.

The bulk density was derived from the total weight and volume of the diluted sludge sample in the $100 \mathrm{~mL}$ graduated cylinder. The liquid phase density, determined by analysis, was used to calculate the weight of the supernatant liquid in the graduated cylinder, which gives the weight of the settled solids by difference. The settled solids density was calculated from the measured volume and calculated weight of the settled solids.

\subsection{PHYSICAL PROPERTY MEASUREMENTS}

Shear strength measurements were performed on the undisturbed samples prior to the WTC sample preparation. For the shear strength measurements, the Haake M5 Viscometer was equipped with a FL- 1000 shear vane. Shear strength is defined as the largest torque measured as the shear vane is rotated a slow constant shear rate. Laboratory analytical procedure LA-519-105 was followed for the shear strength analyses.

Viscosity measurements were performed on the undiluted composite sample and the five dilution levels. The viscosity was also measured for the supernatant liquid from the diluted sludge at the target dilution. A Haake M5 Viscometer was used to perform all viscosity measurements. The Haake M5 viscometer was equipped with the MV-1 coaxial sensor system. Viscosity is calculated by measuring the torque on the inner cylinder as a function of its rotational speed. Viscosity measurements were made at ambient hot cell temperature, $45^{\circ} \mathrm{C}$, and $65^{\circ} \mathrm{C}$. Laboratory analytical procedure LA-519-105 was followed for the viscosity analyses.

Particle size distribution analyses were performed on the undiluted composite and the primary three dilution levels $(60 \mathrm{~g} / \mathrm{L}, 100 \mathrm{~g} / \mathrm{L}$, and $140 \mathrm{~g} / \mathrm{L})$. A Horiba LA-910 Particle Size Analyzer was used for all particle size distribution measurements. The Horiba LA-910 uses laser diffraction to determine the volume distribution of particles with sizes ranging from $0.02 \mu \mathrm{m}$ to $1020 \mu \mathrm{m}$. The particles were diluted with enough supernatant liquid to make a well-separated suspension. All measurements were performed at ambient laboratory temperature (typically around $25^{\circ} \mathrm{C}$ ). Laboratory technology procedure LT-519-105 was followed for the particle size distribution analyses. 
RPP-5798, Rev. 0

\subsection{SLUDGE WASHING AND CAUSTIC LEACHING}

According to the test plan, sludge washing and caustic leaching tests were to be performed on samples of centrifuged sludge (Herting et al 1999). These tests were not performed because of direction from River Protection Project (RPP) to suspend all sludge washing activities (Powell 1999).

\subsection{SLUDGE COMPOSITION AS A FUNCTION OF SETTLING}

This portion of the study could not be performed precisely as specified in the test plan. The test plan called for duplicate samples of the sludge at the target dilution level to be poured into $100 \mathrm{~mL}$ graduated cylinders and allowed to settle. When one-third of the solids had settled to the bottom, the remaining slurry would have been transferred to new graduated cylinders and allowed to settle. When one-half of the remaining solids settled, the remaining slurry would have been decanted into centrifuge cones. The first and second fractions would have also been'transferred from the graduated cylinders to centrifuge cones (Beck 1999).

The test plan assumed distinct slurry and settled solids layers would be visible. Unfortunately, there was no obvious interface between the slurry and settled solids. As an alternative, the following procedure was followed to collect the three fractions. The slurry was allowed to settle for twenty-four hours after initially being transferred into the graduated cylinder. This time period was selected based upon the results of the settling study which showed most of the settling taking place with the first twenty-four hours. Then supernatant liquid and top two-thirds of the slurry were transferred into new graduated cylinders. This slurry was allowed to settle for another twenty-four hours. The supernatant liquid and upper half of the slurry were transferred into a $50 \mathrm{~mL}$ centrifuge cone. The other two fractions were also transferred into $50 \mathrm{~mL}$ centrifuge cones. Thus, the original slurry was divided into three fractions (in duplicate), with the fractions differentiated by the settling rate of the solids. All of the cones were centrifuged, and solids were analyzed for the following: TGA and particle size distribution (direct sample); and ICP/AES, GEA, and AT/TB (after fusion) (Herting et al 1999). 
RPP-5798, Rev. 0

\subsection{RESULTS OF ANALYTICAL MEASUREMENTS}

\subsection{WHOLE-TANK-COMPOSITE TECHNETIUM-99 ANALYSIS}

The ${ }^{99} \mathrm{Tc}$ analytical results (both ICP/MS and liquid scintillation) for the undiluted WTC sample are presented in Table 3-1. The sample was prepared for the ${ }^{99} \mathrm{Tc}$ analyses by potassium hydroxide fusion.

The analytical results of the two methods were both reported as "less than" values, which makes a direct comparison difficult. A report recently examined the reliability of several technetium analytical methods. Instances were cited where the liquid scintillation method provided results orders of magnitude higher or lower than those of the ICP/MS method. The authors attributed these differences to either interferences or incomplete oxidation associated with the liquid scintillation method. In general, ICP/MS was thought to be the more accurate and reliable method. The authors recommended both methods continue to be used for characterization of waste (Troyer and Winters 1999).

Table 3-1 Technetium-99 Analysis of WTC Sample

\begin{tabular}{|l|c|c|}
\hline \multicolumn{1}{|c|}{ Analyte } & Unit & Result \\
\hline $\begin{array}{l}\text { Technetium-99 } \\
\text { by Liquid Scintillation }\end{array}$ & $\mu \mathrm{Ci} / \mathrm{g}$ & $<1.43 \mathrm{e}-02$ \\
\hline Technetium-99 by ICP/MS & $\mu \mathrm{Ci} / \mathrm{g}$ & $<2.75 \mathrm{e}-02$ \\
\hline
\end{tabular}

\subsection{PHYSICAL PROPERTY MEASUREMENTS}

\subsubsection{Shear Strength Results}

Table 3-2 contains the shear strengths measured on the samples collected from core 247 . Prior to the shear strength measurements, the samples had been removed from their core samplers and left undisturbed in storage jars for approximately six months. As would be expected, the shear strengths tend to increase with the sample depth in the tank.

The shear strength could not be measured for the two samples collected from segment 5 . These two samples were sufficiently hard that the shear vane could not be pushed into the sample material. 
Table 3-2 Results of Shear Strength Measurements

\begin{tabular}{|c|c|c|}
\hline Jar Number & Segment Number & Shear Strength \\
\hline 13596 & 1 Upper Solids & $37 \mathrm{~Pa}$ \\
\hline 13607 & 1 Lower Solids & $31 \mathrm{~Pa}$ \\
\hline 14737 & 2 Upper Solids & $56 \mathrm{~Pa}$ \\
\hline 14754 & 2 Lower Solids & $260 \mathrm{~Pa}$ \\
\hline 13017 & 3 Quarter A & $95 \mathrm{~Pa}$ \\
\hline 13018 & 3 Quarter B & $140 \mathrm{~Pa}$ \\
\hline 13019 & 3 Quarter C & $85 \mathrm{~Pa}$ \\
\hline 13020 & 3 Quarter D & $280 \mathrm{~Pa}$ \\
\hline 12396 & 4 Upper Solids & $87 \mathrm{~Pa}$ \\
\hline 12395 & 4 Lower Solids & $760 \mathrm{~Pa}$ \\
\hline 13012 & 5 Upper Solids & Not Measured \\
\hline 13013 & 5 Lower Solids & Not Measured \\
\hline
\end{tabular}

\subsubsection{Viscosity Results}

The viscosity measurements were performed with shear rates increasing from $0 \mathrm{~s}^{-1}$ to $300 \mathrm{~s}^{-1}$ and decreasing from $300 \mathrm{~s}^{-1}$ to $0 \mathrm{~s}^{-1}$. The operating range for the viscometer varies as a function of the viscosity of the material. Table 3-3 lists the lower end of the operating range at several different viscosities. Measurements with shear rates below the lower operating limit should considered highly questionable.

Table 3-3 - Lower Operating Range of Haake M5 Viscometer

\begin{tabular}{|c|c|}
\hline Viscosity & $\begin{array}{c}\text { Lower } \\
\text { Operating } \\
\text { Limit }\end{array}$ \\
\hline $10 \mathrm{cP}$ & $\sim 100 \mathrm{~s}^{-1}$ \\
\hline $25 \mathrm{cP}$ & $\sim 75 \mathrm{~s}^{-1}$ \\
\hline $50 \mathrm{cP}$ & $\sim 50 \mathrm{~s}^{-1}$ \\
\hline
\end{tabular}

Table 3-4 provides a summary of the mean viscosities observed during each of the sample runs. The mean viscosities were calculated after excluding measurements made outside of the operating range of the instrument. Appendix A contains plots of shear stress $(\tau)$ vs. shear rate $(\gamma)$ and viscosity $(\eta)$ vs. shear rate $(\gamma)$ for each of the measurements. Appendix A also contains raw data from each of the sample runs. 
Each of the WTC and concentrated slurry samples had a measurable yield stress. Yield stress can be defined as the amount of force required for the transition of a material from solid or gel behavior to that of a fluid. The rheogram of a material with a yield stress is characterized by a near vertical line at the origin. The rheogram then angles sharply to the right when sufficient force has been transmitted to make the material yield. Table 3-4 provides the yield stresses observed in the rheograms for samples with high solids content.

The supernatant liquid and more dilute slurry samples generally exhibited the Newtonian behavior of viscosity remaining constant with shear rate. Much of the "noisiness" of the viscosity measurements (shown in Appendix A) can be attributed to the relatively low viscosities (less than $10 \mathrm{cP}$ ) for many of the samples.

Table 3-4 Summary of Viscosity Measurements

\begin{tabular}{|c|c|c|c|}
\hline Dilution & Temperature & $\begin{array}{c}\text { Mean Viscosity } \\
( \pm 20 \%)\end{array}$ & $\begin{array}{c}\text { Yield Stress } \\
( \pm 20 \%)\end{array}$ \\
\hline \multirow{3}{*}{ Decanted Supernatant Liquid } & Ambient & $1.7 \mathrm{cP}$ & None \\
\cline { 2 - 4 } & $45^{\circ} \mathrm{C}$ & $1.6 \mathrm{cP}$ & None \\
\cline { 2 - 4 } & $65^{\circ} \mathrm{C}$ & $1.5 \mathrm{cP}$ & None \\
\hline \multirow{3}{*}{ Dilution Level "-" (60 g solids/L) } & Ambient & $2.6 \mathrm{cP}$ & None \\
\cline { 2 - 4 } & $45^{\circ} \mathrm{C}$ & $1.5 \mathrm{cP}$ & None \\
\cline { 2 - 4 } & $65^{\circ} \mathrm{C}$ & $1.3 \mathrm{cP}$ & None \\
\hline \multirow{3}{*}{ Target Dilution (100 g solids/L) } & Ambient & $3.2 \mathrm{cP}$ & None \\
\cline { 2 - 4 } & $45^{\circ} \mathrm{C}$ & $2.4 \mathrm{cP}$ & None \\
\cline { 2 - 4 } & $65^{\circ} \mathrm{C}$ & $1.8 \mathrm{cP}$ & None \\
\hline \multirow{3}{*}{ Dilution Level "+" (140 g solids/L) } & Ambient & $5.5 \mathrm{cP}$ & None \\
\cline { 2 - 4 } & $45^{\circ} \mathrm{C}$ & $4.6 \mathrm{cP}$ & None \\
\cline { 2 - 4 } & $65^{\circ} \mathrm{C}$ & $3.7 \mathrm{cP}$ & None \\
\hline \multirow{3}{*}{ High Solids Dilution (200 g solids/L) } & Ambient & $8.0 \mathrm{cP}$ & $0.8 \mathrm{~Pa}$ \\
\cline { 2 - 4 } & $45^{\circ} \mathrm{C}$ & $7.9 \mathrm{cP}$ & $1.0 \mathrm{~Pa}$ \\
\cline { 2 - 4 } & $65^{\circ} \mathrm{C}$ & $7.3 \mathrm{cP}$ & $1.3 \mathrm{~Pa}$ \\
\hline \multirow{3}{*}{ High Solids Dilution (300 g solids/L) } & Ambient & $29.7 \mathrm{cP}$ & $2.9 \mathrm{~Pa}$ \\
\cline { 2 - 4 } & $45^{\circ} \mathrm{C}$ & $31.2 \mathrm{cP}$ & $4.0 \mathrm{~Pa}$ \\
\cline { 2 - 4 } & $65^{\circ} \mathrm{C}$ & $32.5 \mathrm{cP}$ & $5.0 \mathrm{~Pa}$ \\
\hline Whole-Tank-Composite & Ambient & $43.6 \mathrm{cP}$ & $5.0 \mathrm{~Pa}$ \\
\hline (620 g solids/L) & $45^{\circ} \mathrm{C}$ & $23.5 \mathrm{cP}$ & $4.2 \mathrm{~Pa}$ \\
\cline { 2 - 4 } & $65^{\circ} \mathrm{C}$ & $18.9 \mathrm{cP}$ & $3.4 \mathrm{~Pa}$ \\
\hline
\end{tabular}

In Figure 3-1, the viscosities are plotted as a function of solids concentration. The plot indicates a dramatic increase in viscosity occurring between the $200 \mathrm{~g} / \mathrm{L}$ and $300 \mathrm{~g} / \mathrm{L}$ dilution levels. 
Figure 3-1 also emphasizes some inconsistencies in the viscosity meaurements for the more concentrated slurries. For the $300 \mathrm{~g} / \mathrm{L}$ dilution level, the mean viscosity increased as the temperature was raised from ambient hot cell temperature to $65^{\circ} \mathrm{C}$. Additionally, the $300 \mathrm{~g} / \mathrm{L}$ dilution level had higher mean viscosities at elevated temperatures than the WTC $(620 \mathrm{~g} / \mathrm{L})$. Clearly, the viscosity of a material would be expected to decrease with increased temperature and to increase with increased solids concentration.

\section{Figure 3-1 Viscosity as a Function of Solids Concentration}

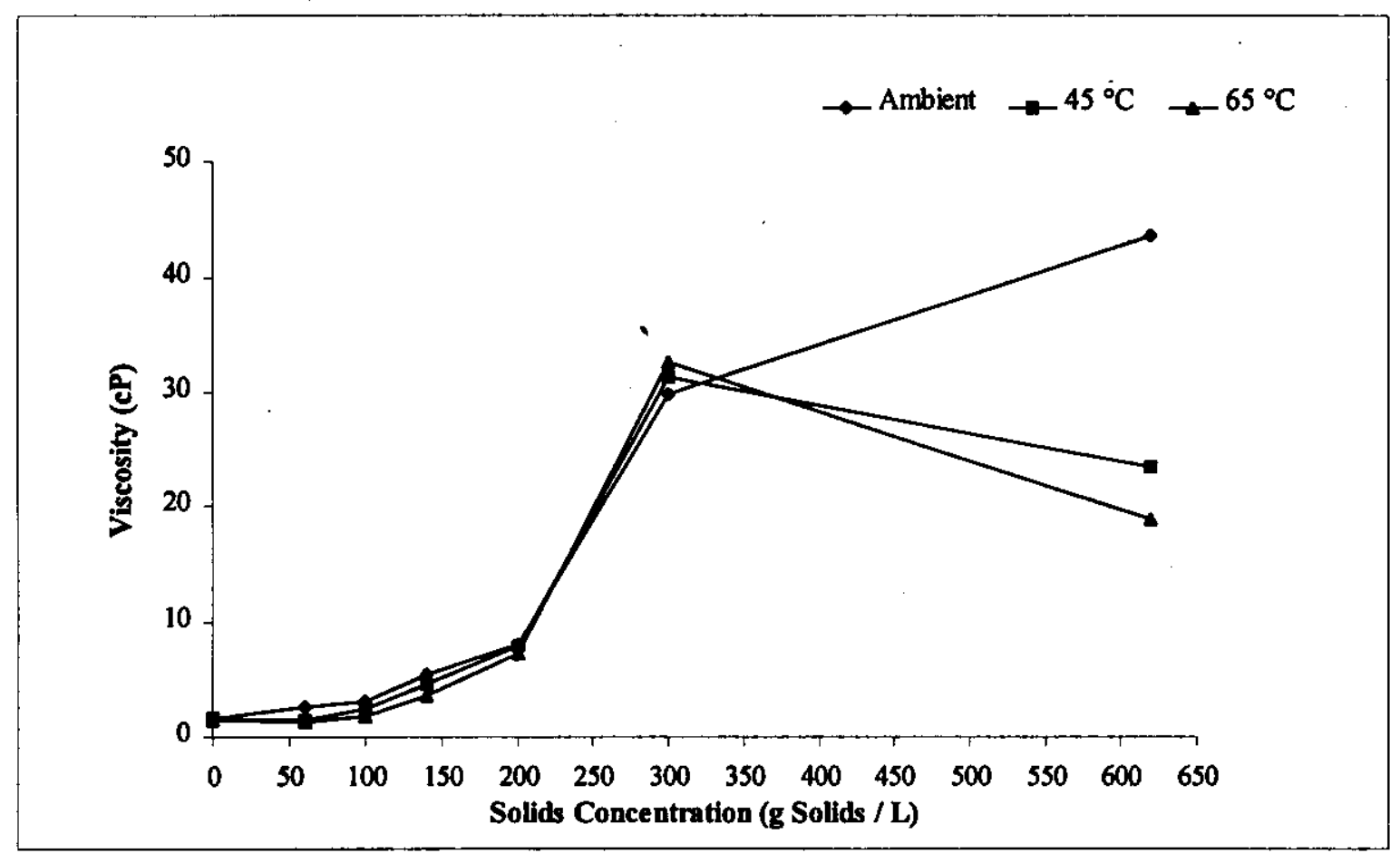

\subsubsection{Particle Size Distribution Results}

Particle size distribution analyses were performed on the undiluted composite and the primary three dilution levels $(60 \mathrm{~g} / \mathrm{L}, 100 \mathrm{~g} / \mathrm{L}$, and $140 \mathrm{~g} / \mathrm{L})$. Table $3-5$ provides a summary of the means and ranges for each of the sample runs. Appendix $B$ contains the particle size distribution data in graphical and tabular form.

The WTC, $100 \mathrm{~g} / \mathrm{L}$ dilution level, and $140 \mathrm{~g} / \mathrm{L}$ dilution level samples have remarkably similar distributions. The $100 \mathrm{~g} / \mathrm{L}$ and $140 \mathrm{~g} / \mathrm{L}$ dilution levels had virtually identical distribution of particles. The WTC sample had somewhat smaller range of particles. 
The $60 \mathrm{~g} / \mathrm{L}$ dilution level sample had a very unusual distribution, with $93 \%$ of the particle mass between 890 and $1020 \mu \mathrm{m}$ and the remainder between 35 and $230 \mu \mathrm{m}$. The Horiba LA-910 measures the distribution based upon the volume of particles, which means a relatively small number of $1000 \mu \mathrm{m}$ particles could have obscured the particles in the $100 \mu \mathrm{m}$ range.

The particles may have undergone size changes during sampling, storage, or processing before the measurements were performed. The particle size distribution of the sample that was analyzed does not necessarily represent in-situ conditions.

Table 3-5 Summary of Particle Size Distribution Measurements

\begin{tabular}{|c|c|c|c|}
\hline Dilution & File Name & Mean & Range \\
\hline Whole-Tank-Composite & C104WTS & $70 \mu \mathrm{m}$ & $5-175 \mu \mathrm{m}$ \\
\hline Dilution Level "-" (60 g solids/L) & C104T- & $900 \mu \mathrm{m}$ & $35-1020 \mu \mathrm{m}$ \\
\hline Target Dilution (100 g solids/L) & C104T0 & $100 \mu \mathrm{m}$ & $6-200 \mu \mathrm{m}$ \\
\hline Dilution Level "+" (140 g solids/L) & C104TPOS & $100 \mu \mathrm{m}$ & $6-200 \mu \mathrm{m}$ \\
\hline
\end{tabular}

\subsection{DENSITY AND SETTLING RATE MEASUREMENTS}

\subsubsection{Density Measurements}

Densities were determined for the bulk sample, liquid phase, and settled solids for each of the three dilution levels $(60 \mathrm{~g} / \mathrm{L}, 100 \mathrm{~g} / \mathrm{L}$, and $140 \mathrm{~g} / \mathrm{L})$ of the composite sample. The density measurements were obtained from samples used for settling rate tests.

Table 3-6 contains the weights, volumes, and densities for each of the three dilution levels. The bulk density was derived from the total weight and volume for each diluted sludge sample. Supernatant liquid samples were analyzed for specific gravity. The liquid phase density was used to calculate the weight of the supernatant liquid in each graduated cylinder, which gives the weight of the settled solids by difference. The settled solids density was calculated from the measured volume and calculated weight of the settled solids.

With one exception, the density measurements increased with the solids concentration. For the settled solids, the density of the $60 \mathrm{~g} / \mathrm{L}$ dilution level was slightly higher than $100 \mathrm{~g} / \mathrm{L}$ dilution level. The difference is probably within the cumulative measurement error for this density calculation. 
Table 3-6 Density Measurements

\begin{tabular}{|c|c|c|c|c|}
\hline & Measurement & $60 \mathrm{~g} / \mathrm{L}$ Slurry & $100 \mathrm{~g} / \mathrm{L}$ Slurry & $140 \mathrm{~g} / \mathrm{L}$ Slurry \\
\hline \multirow{3}{*}{ Bulk Material } & Weight & $106.8 \mathrm{~g}$ & $109.3 \mathrm{~g}$ & $115.3 \mathrm{~g}$ \\
\cline { 2 - 5 } & Volume & $100.0 \mathrm{~mL}$ & $99.0 \mathrm{~mL}$ & $99.5 \mathrm{~mL}$ \\
\cline { 2 - 5 } & Density & $1.068 \mathrm{~g} / \mathrm{mL}$ & $1.104 \mathrm{~g} / \mathrm{ml}$ & $1.159 \mathrm{~g} / \mathrm{mL}$ \\
\hline $\begin{array}{c}\text { Supernatant } \\
\text { Liquid }\end{array}$ & Weight & $81.3 \mathrm{~g}$ & $67.6 \mathrm{~g}$ & $61.0 \mathrm{~g}$ \\
\cline { 2 - 5 } & Volume & $79.5 \mathrm{~mL}$ & $65.0 \mathrm{~mL}$ & $58.0 \mathrm{~mL}$ \\
\cline { 2 - 5 } & Density & $1.023 \mathrm{~g} / \mathrm{mL}$ & $1.040 \mathrm{~g} / \mathrm{mL}$ & $1.052 \mathrm{~g} / \mathrm{mL}$ \\
\hline \multirow{3}{*}{ Settled Solids } & $25.5 \mathrm{~g}$ & $41.7 \mathrm{~g}$ & $54.3 \mathrm{~g}$ \\
\cline { 2 - 5 } & Weight & $20.5 \mathrm{~mL}$ & $34.0 \mathrm{~mL}$ & $41.5 \mathrm{~mL}$ \\
\cline { 2 - 5 } & Volume & $1.243 \mathrm{~g} / \mathrm{mL}$ & $1.226 \mathrm{~g} / \mathrm{mL}$ & $1.308 \mathrm{~g} / \mathrm{mL}$ \\
\cline { 2 - 6 } & Density & \multicolumn{3}{|c}{} \\
\hline
\end{tabular}

\subsubsection{Bulk Density of Undiluted Sludge}

The bulk density of the undiluted sludge was calculated from the bulk density of the diluted samples by accounting for the water additions. The calculations are shown below for the target dilution, where IW $=$ inhibited water and $\mathrm{TD}=$ target dilution.

The preparation of the target dilution samples was discussed in Section 2.2.1. The amount of inhibited water present in the graduated cylinder used in the settling study can be calculated as follows.

$$
109.3 \mathrm{~g} \mathrm{TD} \times \frac{370.1 \mathrm{~g} \mathrm{IW}}{505.4 \mathrm{~g} \mathrm{TD}}=80.0 \mathrm{~g} \mathrm{IW}
$$

The bulk density of the undiluted sludge can be calculated by subtracting the inhibited water from both the weights and volumes of the target dilution sample.

$$
\frac{109.3 \mathrm{~g} \mathrm{TD}-80.0 \mathrm{~g} \mathrm{IW}}{99.0 \mathrm{~mL} \mathrm{TD}-80.0 \mathrm{~mL} \mathrm{IW}}=1.54 \mathrm{~g} / \mathrm{mL} \text { Undiluted Sludge }
$$

Similar calculations performed for the $60 \mathrm{~g} / \mathrm{L}$ and $140 \mathrm{~g} / \mathrm{L}$ dilution level samples yielded values of $1.50 \mathrm{~g} / \mathrm{mL}$ and $1.62 \mathrm{~g} / \mathrm{mL}$, respectively. The mean bulk density of the undiluted sludge is $1.55 \mathrm{~g} / \mathrm{mL}$. These density values are lower than the $1.67 \mathrm{~g} / \mathrm{mL}$ reported previously (Baldwin1997, Table B3-5).

The bulk density calculation does not account for the dissolution of solids after dilution. The solids dissolution would have only a minor effect on the final result, well within the analytical error for this calculation. 


\subsubsection{Settling Rate Measurements}

In Figure 3-2, the position of the slurry-liquid interface is shown for the three dilution levels over the duration of one week. Table 3-7 shows the slurry-liquid interface over the course of the settling tests. The three dilution conditions had the characteristic settling shape of rapid, unhindered settling followed by a longer period of compaction.

In The Settling and Compaction of Nuclear Waste Slurries, the author evaluated the settling of tank waste simulant in several different sized vessels. The studies performed in $100 \mathrm{~mL}$ graduated cylinders were found to adequately represent the initial phase of rapid settling. The tests in $100 \mathrm{~mL}$ graduated cylinders failed to achieve the same degree of compaction observed in larger vessels (MacLean 1999).

Figure 3-2 Settling Study with Diluted Sludge

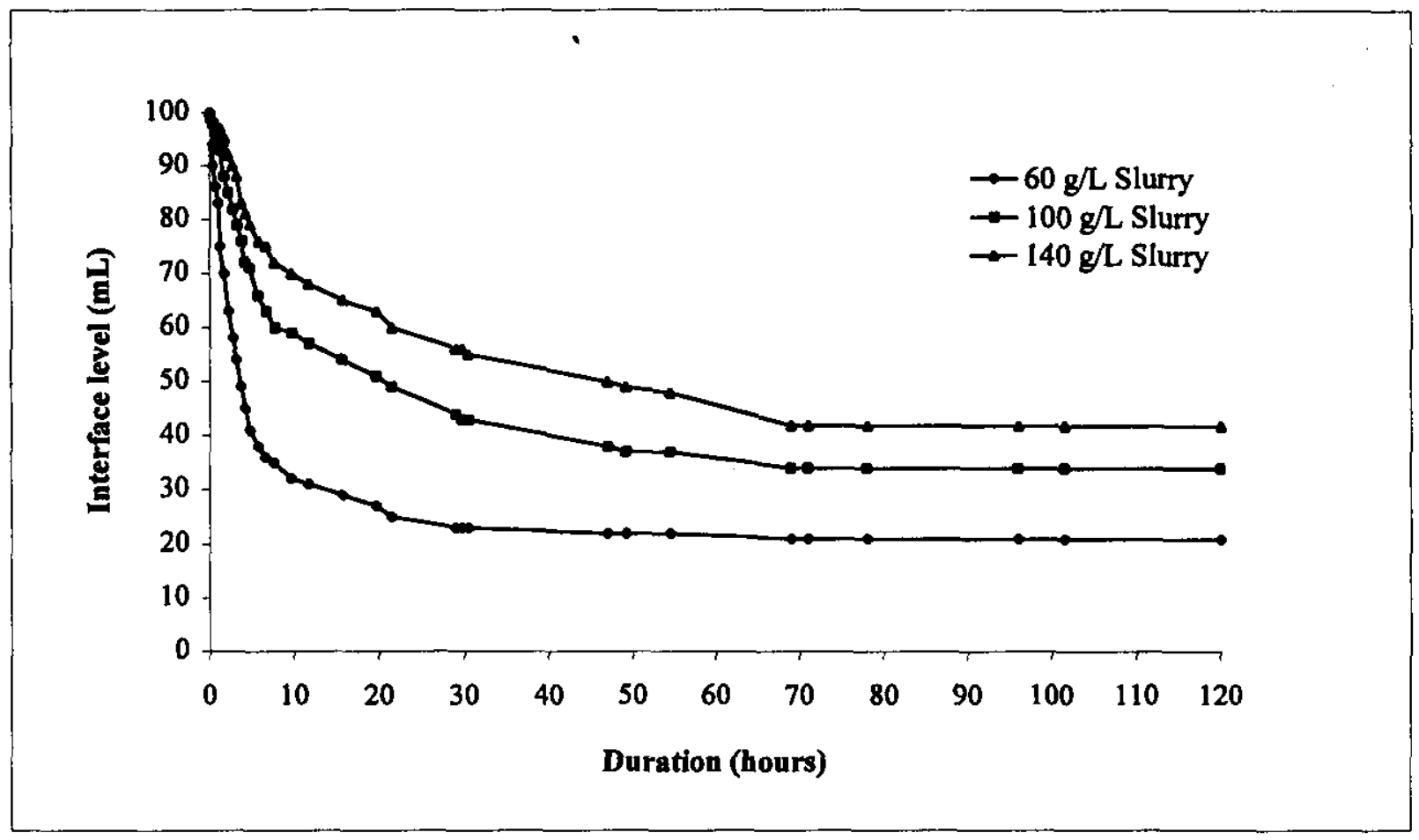

\subsection{TARGET DILUTION CHEMICAL COMPOSITION}

Duplicate samples of the liquid and solid phases were submitted for analysis after centrifugation. No separable organic phase was apparent after 30 minutes of centrifugation. Despite the centrifugation, samples of the solid phase will contain some interstitial liquid. The results of the chemical analyses for the supernatant liquid and centrifuged solids are presented in Tables 3-9 and 3-10, respectively. 
RPP-5798, Rev. 0

Table 3-7 Slurry-Liquid Interface Levels

\begin{tabular}{|c|c|c|c|}
\hline $\begin{array}{c}\text { Duration } \\
\text { (hours) }\end{array}$ & $\begin{array}{c}60 \mathrm{~g} / \mathrm{L} \\
\text { Slurry }(\mathrm{cm})\end{array}$ & $\begin{array}{c}100 \mathrm{~g} / \mathrm{L} \\
\text { Slurry }(\mathrm{cm})\end{array}$ & $\begin{array}{c}140 \mathrm{~g} / \mathrm{L} \\
\text { Slurry }(\mathrm{cm})\end{array}$ \\
\hline 0.0 & 100 & 99 & 100 \\
\hline 0.3 & 94 & 98 & 98 \\
\hline 0.4 & 90 & 98 & 98 \\
\hline 0.7 & 86 & 97 & 98 \\
\hline 1.0 & 83 & 95 & 97 \\
\hline 1.2 & 75 & $\overline{93}$ & 97 \\
\hline 1.7 & 70 & 88 & 95 \\
\hline 2.2 & 63 & $\overline{85}$ & 92 \\
\hline 2.7 & 58 & 82 & 90 \\
\hline 3.2 & 54 & 79 & 88 \\
\hline 3.7 & 49 & $\overline{76}$ & $\overline{83}$ \\
\hline 4.2 & 45 . & 72 & 81 \\
\hline 4.7 & 41 & 71 & 79 \\
\hline 5.7 & $\overline{38}$ & 66 & 76 \\
\hline 6.7 & 36 & 63 & 75 \\
\hline 7.7 & 35 & 60 & 72 \\
\hline 9.7 & $\overline{32}$ & 59 & $\overline{70}$ \\
\hline 12 & $\overline{31}$ & 57 & 68 \\
\hline 16 & 29 & $\overline{54}$ & 65 \\
\hline 20 & 27 & 51 & 63 \\
\hline 22 & 25 & 49 & 60 \\
\hline 29 & 23 & 44 & 56 \\
\hline 30 & 23 & 43 & 56 \\
\hline 31 & 23 & $\overline{43}$ & 55 \\
\hline 47 & 22 & 38 & 50 \\
\hline 49 & 22 & $\overline{37}$ & 49 \\
\hline 55 & 22 & 37 & 48 \\
\hline 69 & 21 & $\overline{34}$ & 42 \\
\hline 71 & 21 & $\overline{34}$ & 42 \\
\hline 78 & 21 & $\overline{34}$ & 42 \\
\hline 96 & 21 & 34 & 42 \\
\hline 102 & 21 & $\overline{34}$ & 42 \\
\hline 120 & 21 & 34 & 42 \\
\hline
\end{tabular}


Some analytical values that were reported were unrealistic, i.e., not consistent with clearand expected trends established by the preponderance of the data. Those values are included in the tables, but are designated by placing the values inside parentheses 0 .

Mass and charge balance calculations can be done on the supernatant liquid and centrifuged solids fractions. The formula used for the mass balance calculation of the liquid fraction is:

$$
\text { Mass Balance }=\% \mathrm{H}_{2} \mathrm{O}+\sum_{\mathrm{i}} \mathrm{m}_{\mathrm{i}} \mathrm{C}_{\mathrm{i}} /(10,000 \times \text { Density })
$$

where $C_{i}$ values are concentrations expressed in $\mu \mathrm{g} / \mathrm{mL}$ as shown in Table 3-9; $m_{i}$ are multiplication factors to account for the actual masses of the components; and density is in $\mathrm{g} / \mathrm{mL}$. The formula for the mass balance calculation of the solid fraction is:

$$
\text { Mass Balance }=\% \mathrm{H}_{2} \mathrm{O}+\sum_{\mathrm{i}} \mathrm{m}_{\mathrm{i}} \mathrm{C}_{\mathrm{i}} / 10,000
$$

where $C_{i}$ values are concentrations expressed in $\mu \mathrm{g} / \mathrm{g}$ as shown in Table 3-10 and $\mathrm{m}_{\mathrm{i}}$ are the multiplication factors. The multiplication factors are listed in Table 3-8.

Table 3-8 Multiplication Factors for Mass Balance Calculation

\begin{tabular}{|c|c|c|}
\hline Analyte & $\begin{array}{c}\text { Assumed } \\
\text { Compound }\end{array}$ & $\begin{array}{c}\text { Multiplication } \\
\text { Factor }\end{array}$ \\
\hline $\mathrm{OH}^{-}$ & $\mathrm{OH}^{-}$ & 1 \\
\hline $\mathrm{TOC}$ & Complexant & 3 \\
\hline $\mathrm{TIC}$ & $\mathrm{CO}_{3}$ & 5 \\
\hline $\mathrm{Al}$ & $\mathrm{Al}_{2} \mathrm{O}_{3}$ & 1.9 \\
\hline $\mathrm{Ca}$ & $\mathrm{CaO}^{-}$ & 1.4 \\
\hline $\mathrm{Cr}$ & $\mathrm{Cr}_{2} \mathrm{O}_{3}$ & 1.5 \\
\hline $\mathrm{Fe}$ & $\mathrm{Fe}_{2} \mathrm{O}_{3}$ & 1.4 \\
\hline $\mathrm{Mn}$ & $\mathrm{MnO}_{2}$ & 1.6 \\
\hline $\mathrm{Ni}$ & $\mathrm{NiO}^{-}$ & 1.3 \\
\hline $\mathrm{Si}$ & $\mathrm{SiO}_{2}$ & 2.1 \\
\hline $\mathrm{U}$ & $\mathrm{U}$ & 1 \\
\hline $\mathrm{Zr}$ & $\mathrm{ZrO}_{2}$ & 1.4 \\
\hline $\mathrm{F}^{-}$ & $\mathrm{F}^{-}$ & 1 \\
\hline $\mathrm{Cl}$ & $\mathrm{Cl}^{-}$ & 1 \\
\hline $\mathrm{NO}_{2}^{-}$ & $\mathrm{NO}_{2}^{-}$ & 1 \\
\hline $\mathrm{NO}_{3}^{-}$ & $\mathrm{NO}_{3}^{-}$ & 1 \\
\hline $\mathrm{PO}_{4}^{3-}$ & $\mathrm{PO}_{4}^{3-}$ & 1 \\
\hline $\mathrm{SO}_{4}^{2-}$ & $\mathrm{SO}_{4}^{2-}$ & 1 \\
\hline
\end{tabular}


The TOC concentrations were larger than those of oxalate and were used for the mass balance calculations. The large discrepancy between the TOC and oxalate concentrations in the solid phase indicates the presence of a different organic species.

A multiplication factor of 3 was selected for the TOC concentration to account for the mass of oxygen, nitrogen, and hydrogen associated with the carbon in typical organic . complexants. Uranium was assumed to be in the metallic form because of the negligible mass of oxygen relative to the mass of uranium.

The formula used for the charge balance calculation is:

$$
\text { Charge Balance }=\sum_{\mathrm{i}}\left(\mathrm{C}_{+} / \mathrm{W}_{+}\right) / \sum_{\mathrm{i}}\left(\mathrm{C}_{-} \mathrm{Z}_{-} / \mathrm{W}_{-}\right)
$$

where $\mathrm{C}_{+}$values are concentrations of $\mathrm{Na}$ and $\mathrm{K}$ in $\mu \mathrm{g} / \mathrm{mL} ; \mathrm{W}_{+}$are the respective atomic weights; $\mathrm{C}$. are the concentrations of anionic species $\left(\mathrm{OH}\right.$, TIC, TOC, $\mathrm{Al}, \mathrm{F}^{-}, \mathrm{Cl}^{-}, \mathrm{NO}_{2}^{-}$, $\left.\mathrm{NO}_{3}^{-}, \mathrm{PO}_{4}^{3-}, \mathrm{SO}_{4}^{2-}\right) ; \mathrm{Z}$. are the charges on the respective ions; and W. are the formula weights of the anions. The $\mathrm{Z}$. for TOC is taken to be 0.5 , assuming a typical mixture of organic compounds in solution that have an average of two carbon atoms per negative charge. The Z for TIC is 2 . Aluminum is assumed to be present as aluminate ion, $\mathrm{Al}(\mathrm{OH})_{4}^{-}$, but 27 is used as the formula weight because the concentration is expressed in the tables as $\mathrm{Al}$, not $\mathrm{Al}(\mathrm{OH})_{4}^{-}$.

When the data is reliable, mass balance values should be near $100 \%$. Charge balance values should be near 1.00 , but are typically lower (averaging approximately $95 \%$ ) for unknown reasons (Herting 1999).

The mass balance values for the supernatant liquid samples were excellent. The charge balance values for the liquid samples were lower than expected. The charge balance for the duplicate sample was skewed by an unrealistic hydroxide concentration. The charge balance for the duplicate sample would have been 0.88 if the hydroxide concentration were an order of magnitude lower. While the mass and charge balances for the centrifuged solids sample were satisfactory, the values for the duplicate sample indicate inconsistencies within the data set.

Two separate preparation methods were used for the ICP/AES analyses of the solid phase samples. Table 3-11 contains a comparison of the two sets of data. With the exception of silicon, the two sets of data are in very good agreement for all of the metals. The silicon analyses were an order of magnitude higher after the fusion preparation method. Nickel and potassium were not reported for the samples prepared by the fusion method, which uses nickel crucibles with the addition of potassium hydroxide. 
RPP-5798, Rev. 0

Table 3-9 Composition of Target Dilution Supernatant Liquid (page 1 of 2)

\begin{tabular}{|l|c|c|c|}
\hline \multicolumn{1}{|c|}{ Analyte } & Unit & TD Liquid & $\begin{array}{c}\text { TD Liquid } \\
\text { (Duplicate) }\end{array}$ \\
\hline Specific Gravity & Sp.G. & 1.057 & 1.041 \\
\hline \% Water by TGA & $\%$ & 93.67 & 93.77 \\
\hline OH & $\mu \mathrm{g} / \mathrm{mL}$ & $8.96 \mathrm{e}+02$ & $(8.59 \mathrm{e}+03)$ \\
\hline pH & $\mathrm{pH}$ & 12.32 & 12.43 \\
\hline TIC & $\mu \mathrm{g} / \mathrm{mL}$ & $1.32 \mathrm{e}+03$ & $1.28 \mathrm{e}+03$ \\
\hline TOC & $\mu \mathrm{g} / \mathrm{mL}$ & $2.23 \mathrm{e}+03$ & $1.41 \mathrm{e}+03$ \\
\hline Silver & $\mu \mathrm{g} / \mathrm{mL}$ & 1.63 & 2.41 \\
\hline Aluminium & $\mu \mathrm{g} / \mathrm{mL}$ & $1.84 \mathrm{e}+02$ & $1.84 \mathrm{e}+02$ \\
\hline Arsenic & $\mu \mathrm{g} / \mathrm{mL}$ & $<10.1$ & $<10.1$ \\
\hline Boron & $\mu \mathrm{g} / \mathrm{mL}$ & $<5.05$ & $<5.05$ \\
\hline Barium & $\mu \mathrm{g} / \mathrm{mL}$ & $<5.05$ & $<5.05$ \\
\hline Beryllium & $\mu \mathrm{g} / \mathrm{mL}$ & $<5.05 \mathrm{e}-01$ & $<5.05 \mathrm{e}-01$ \\
\hline Bismuth & $\mu \mathrm{g} / \mathrm{mL}$ & $<10.1$ & $<10.1$ \\
\hline Calcium & $\mu \mathrm{g} / \mathrm{mL}$ & $<10.1$ & $<10.1$ \\
\hline Cadmium & $\mu \mathrm{g} / \mathrm{mL}$ & $<5.05 \mathrm{e}-01$ & $<5.05 \mathrm{e}-01$ \\
\hline Cerium & $\mu \mathrm{g} / \mathrm{mL}$ & $<10.1$ & $<10.1$ \\
\hline Cobalt & $\mu \mathrm{g} / \mathrm{mL}$ & $<2.02$ & $<2.02$ \\
\hline Chromium & $\mu \mathrm{g} / \mathrm{mL}$ & 17.2 & 16.8 \\
\hline Copper & $\mu \mathrm{g} / \mathrm{mL}$ & $<1.01$ & $<1.01$ \\
\hline Iron & $\mu \mathrm{g} / \mathrm{mL}$ & $<5.05$ & $<5.05$ \\
\hline Potassium & $\mu \mathrm{g} / \mathrm{mL}$ & $1.05 \mathrm{e}+02$ & 98.9 \\
\hline Lanthanum & $\mu \mathrm{g} / \mathrm{mL}$ & $<5.05$ & $<5.05$ \\
\hline Lithium & $\mu \mathrm{g} / \mathrm{mL}$ & $<1.01$ & $<1.01$ \\
\hline Magnesium & $\mu \mathrm{g} / \mathrm{mL}$ & $<10.1$ & $<10.1$ \\
\hline Manganese & $\mu \mathrm{g} / \mathrm{mL}$ & $<1.01$ & $<1.01$ \\
\hline Molybdenum & $\mu \mathrm{g} / \mathrm{mL}$ & $<5.05$ & $<5.05$ \\
\hline Sodium & $\mu \mathrm{g} / \mathrm{mL}$ & $2.37 \mathrm{e}+04$ & $2.30 \mathrm{e}+04$ \\
\hline Neodymium & $\mu \mathrm{g} / \mathrm{mL}$ & $<10.1$ & $<10.1$ \\
\hline Nickel & $\mu \mathrm{g} / \mathrm{mL}$ & 18.4 & 18.6 \\
\hline Phosphorus & $\mu \mathrm{g} / \mathrm{mL}$ & $3.15 \mathrm{e}+02$ & $3.13 \mathrm{e}+02$ \\
\hline Lead & $\mu \mathrm{g} / \mathrm{mL}$ & $<10.1$ & $<10.1$ \\
\hline Sulfur & $\mu \mathrm{g} / \mathrm{mL}$ & $1.98 \mathrm{e}+02$ & $1.93 \mathrm{e}+02$ \\
\hline Antimony & $\mu \mathrm{g} / \mathrm{mL}$ & $<6.06$ & $<6.06$ \\
\hline Selenium & $\mu \mathrm{g} / \mathrm{mL}$ & $<10.1$ & $<10.1$ \\
\hline Silicon & $\mu \mathrm{g} / \mathrm{mL}$ & 76.6 & $2.29 \mathrm{e}+02$ \\
\hline Samarium & $\mu \mathrm{g} / \mathrm{mL}$ & $<10.1$ & $<10.1$ \\
\hline Strontium & $\mu \mathrm{g} / \mathrm{mL}$ & $<1.01$ & $<1.01$ \\
\hline Titanium & $\mu \mathrm{g} / \mathrm{mL}$ & $<1.01$ & $<1.01$ \\
\hline Thallium & $\mu \mathrm{g} / \mathrm{mL}$ & $<20.2$ & $<20.2$ \\
\hline Uranium & $\mu \mathrm{g} / \mathrm{mL}$ & $<50.5$ & $<50.5$ \\
\hline
\end{tabular}


Table 3-9 Composition of Target Dilution Supernatant Liquid (page 2 of 2)

\begin{tabular}{|l|c|c|c|}
\hline Vanadium & $\mu \mathrm{g} / \mathrm{mL}$ & $<5.05$ & $<5.05$ \\
\hline Zinc & $\mu \mathrm{g} / \mathrm{mL}$ & $<1.01$ & $<1.01$ \\
\hline Zirconium & $\mu \mathrm{g} / \mathrm{mL}$ & $<1.01$ & $<1.01$ \\
\hline Mercury & $\mu \mathrm{g} / \mathrm{mL}$ & $2.60 \mathrm{e}-02$ & $2.97 \mathrm{e}-02$ \\
\hline Fluoride & $\mu \mathrm{g} / \mathrm{mL}$ & $1.17 \mathrm{e}+04$ & $1.15 \mathrm{e}+04$ \\
\hline Chloride & $\mu \mathrm{g} / \mathrm{mL}$ & $1.68 \mathrm{e}+02$ & $1.40 \mathrm{e}+02$ \\
\hline Nitrite & $\mu \mathrm{g} / \mathrm{mL}$ & $5.65 \mathrm{e}+03$ & $5.56 \mathrm{e}+03$ \\
\hline Bromide & $\mu \mathrm{g} / \mathrm{mL}$ & $<5.18 \mathrm{e}+02$ & $<5.18 \mathrm{e}+02$ \\
\hline Nitrate & $\mu \mathrm{g} / \mathrm{mL}$ & $3.35 \mathrm{e}+03$ & $3.26 \mathrm{e}+03$ \\
\hline Phosphate & $\mu \mathrm{g} / \mathrm{mL}$ & $1.25 \mathrm{e}+03$ & $(<4.97 \mathrm{e}+02)$ \\
\hline Sulfate & $\mu \mathrm{g} / \mathrm{mL}$ & $7.62 \mathrm{e}+02$ & $8.49 \mathrm{e}+02$ \\
\hline Oxalate & $\mu \mathrm{g} / \mathrm{mL}$ & $1.65 \mathrm{e}+03$ & $1.73 \mathrm{e}+03$ \\
\hline Alpha & $\mu \mathrm{Ci} / \mathrm{mL}$ & $7.40 \mathrm{e}-04$ & $7.87 \mathrm{e}-04$ \\
\hline Beta & $\mu \mathrm{Ci} / \mathrm{mL}$ & 7.1 & 6.88 \\
\hline Cobalt-60 & $\mu \mathrm{Ci} / \mathrm{mL}$ & $7.48 \mathrm{e}-03$ & $7.49 \mathrm{e}-03$ \\
\hline Strontium-89/90 & $\mu \mathrm{Ci} / \mathrm{mL}$ & $2.71 \mathrm{e}-03$ & $2.45 \mathrm{e}-03$ \\
\hline Technetium-99 by & $\mu \mathrm{Ci} / \mathrm{mL}$ & $2.92 \mathrm{e}-03$ & $3.02 \mathrm{e}-03$ \\
ICP/MS & & & \\
\hline Technetium-99 by & $\mu \mathrm{Ci} / \mathrm{mL}$ & $3.23 \mathrm{e}-03$ & $3.02 \mathrm{e}-03$ \\
Liquid Scintillation & & & \\
\hline Antimony-125 & $\mu \mathrm{Ci} / \mathrm{mL}$ & $<5.98 \mathrm{e}-03$ & $<5.88 \mathrm{e}-03$ \\
\hline Tin-126 & $\mu \mathrm{g} / \mathrm{mL}$ & $<6.21 \mathrm{e}-01$ & $<6.21 \mathrm{e}-01$ \\
\hline Cesium-137 & $\mu \mathrm{Ci} / \mathrm{mL}$ & 6.97 & 6.75 \\
\hline Europium-152 & $\mu \mathrm{Ci} / \mathrm{mL}$ & $<2.87 \mathrm{e}-03$ & $<2.82 \mathrm{e}-03$ \\
\hline Europium-154 & $\mu \mathrm{Ci} / \mathrm{mL}$ & $<3.32 \mathrm{e}-04$ & $<3.05 \mathrm{e}-04$ \\
\hline Europium-155 & $\mu \mathrm{Ci} / \mathrm{mL}$ & $<3.33 \mathrm{e}-03$ & $<3.29 \mathrm{e}-03$ \\
\hline Protactinium-231 & $\mu \mathrm{g} / \mathrm{mL}$ & $<8.00 \mathrm{e}-02$ & $<8.00 \mathrm{e}-02$ \\
\hline Uranium-233 & $\mu \mathrm{g} / \mathrm{mL}$ & $<4.97 \mathrm{e}-02$ & $5.63 \mathrm{e}-02$ \\
\hline Uranium-234 & $\mu \mathrm{g} / \mathrm{mL}$ & $<4.97 \mathrm{e}-02$ & $<4.97 \mathrm{e}-02$ \\
\hline Uranium-235 & $\mu \mathrm{g} / \mathrm{mL}$ & $<4.97 \mathrm{e}-02$ & $<4.97 \mathrm{e}-02$ \\
\hline Uranium-236 & $\mu \mathrm{g} / \mathrm{mL}$ & $<6.63 \mathrm{e}-02$ & $<6.63 \mathrm{e}-02$ \\
\hline Uranium-238 & $\mu \mathrm{g} / \mathrm{mL}$ & 2.30 & 3.22 \\
\hline Neptunium-237 & $\mu \mathrm{g} / \mathrm{mL}$ & $<5.39 \mathrm{e}-02$ & $<5.39 \mathrm{e}-02$ \\
\hline Plutonium-239 & $\mu \mathrm{g} / \mathrm{mL}$ & $<3.90 \mathrm{e}-02$ & $<3.90 \mathrm{e}-02$ \\
\hline Plutonium-240 & $\mu \mathrm{g} / \mathrm{mL}$ & $<3.41 \mathrm{e}-02$ & $<3.41 \mathrm{e}-02$ \\
\hline Plutonium-242 & $\mu \mathrm{g} / \mathrm{mL}$ & $<2.32 \mathrm{e}-02$ & $<2.32 \mathrm{e}-02$ \\
\hline Pu/Am-241 & $\mu \mathrm{g} / \mathrm{mL}$ & $<3.61 \mathrm{e}-02$ & $<3.61 \mathrm{e}-02$ \\
\hline Am 243/Cm 243 & $\mu \mathrm{g} / \mathrm{mL}$ & $<1.54 \mathrm{e}-02$ & $<1.54 \mathrm{e}-02$ \\
\hline Pu 244/Cm 244 & $\mu \mathrm{g} / \mathrm{mL}$ & $<3.28 \mathrm{e}-02$ & $<3.28 \mathrm{e}-02$ \\
\hline Mass Balance & $\mathrm{wt} \%$ & 99.5 & 100.1 \\
\hline Charge Balance & --- & 0.86 & 0.63 \\
\hline
\end{tabular}


Table 3-10 Composition of Target Dilution Centrifuged Solids (page 1 of 3)

\begin{tabular}{|c|c|c|c|}
\hline Analyte & Unit & TD Solids & $\begin{array}{c}\text { TD Solids } \\
\text { (Duplicate) }\end{array}$ \\
\hline$\%$ Water by TGA & $\%$ & 55.50 & 51.34 \\
\hline $\mathrm{OH}^{-}$ & $\mu \mathrm{g} / \mathrm{g}$ & $<8.20 \mathrm{e}+03$ & $<8.07 \mathrm{e}+03$ \\
\hline TIC & $\mu \mathrm{g} / \mathrm{g}$ & $1.23 \mathrm{e}+03$ & $1.21 \mathrm{e}+03$ \\
\hline TOC & $\mu \mathrm{g} / \mathrm{g}$ & $7.60 \mathrm{e}+03$ & $7.50 \mathrm{e}+03$ \\
\hline Silver-ICP-Fusion & $\mu \mathrm{g} / \mathrm{g}$ & $6.16 \mathrm{e}+02$ & $5.17 \mathrm{e}+02$ \\
\hline Aluminium-ICP-Fusion & $\mu \mathrm{g} / \mathrm{g}$ & $1.16 \mathrm{e}+\overline{05}$ & $9.80 \mathrm{e}+04$ \\
\hline Arsenic-ICP-Fusion & $\mu \mathrm{g} / \mathrm{g}$ & $<1.93 e+03$ & $<2.04 \mathrm{e}+03$ \\
\hline Boron-ICP-Fusion & $\mu \mathrm{g} / \mathrm{g}$ & $<9.63 \mathrm{e}+02$ & $<1.02 \mathrm{e}+03$ \\
\hline Barium -ICP-Fusion & $\mu \mathrm{g} / \mathrm{g}$ & $<9.63 \mathrm{e}+02$ & $<1.02 \mathrm{e}+03$ \\
\hline Beryllium -ICP-Fusion & $\mu \mathrm{g} / \mathrm{g}$ & $<96.30$ & $<1.02 \mathrm{e}+02$ \\
\hline Bismuth -ICP-Fusion & $\mu \mathrm{g} / \mathrm{g}$ & $6.16 \mathrm{e}+03$ & $5.82 \mathrm{e}+03$ \\
\hline Calcium -ICP-Fusion & $\mu \mathrm{g} / \mathrm{g}$ & $3.30 \mathrm{e}+03$ & $2.79 e+03$ \\
\hline Cadmium -ICP-Fusion & $\mu \mathrm{g} / \mathrm{g}$ & $3.86 \mathrm{e}+02$ & $3.99 \mathrm{e}+02$ \\
\hline Cerium -ICP-Fusion & $\mu \mathrm{g} / \mathrm{g}$ & $<1.93 \mathrm{e}+03$ & $<2.04 \mathrm{e}+03$ \\
\hline Cobalt -ICP-Fusion & $\mu \mathrm{g} / \mathrm{g}$ & $<3.85 \mathrm{e}+02$ & $<4.07 \mathrm{e}+02$ \\
\hline Chromium-ICP-Fusion & $\mu \mathrm{g} / \mathrm{g}$ & $8.48 \mathrm{e}+02$ & $8.66 \mathrm{e}+02$ \\
\hline Copper -ICP-Fusion & $\mu \mathrm{g} / \mathrm{g}$ & $<1.93 e+02$ & $<2.04 \mathrm{e}+02$ \\
\hline Iron -ICP-Fusion & $\mu \mathrm{g} / \mathrm{g}$ & $1.64 \mathrm{e}+04$ & $1.66 \mathrm{e}+04$ \\
\hline Lanthanum -ICP-Fusion & $\mu \mathrm{g} / \mathrm{g}$ & $<9.63 \mathrm{e}+02$ & $<1.02 \mathrm{e}+03$ \\
\hline Lithium -ICP-Fusion & $\mu \mathrm{g} / \mathrm{g}$ & $<1.93 \mathrm{e}+02$ & $<2.04 \mathrm{e}+02$ \\
\hline Magnesium -ICP-Fusion & $\mu \mathrm{g} / \mathrm{g}$ & $<1.93 \mathrm{e}+03$ & $<2.04 \mathrm{e}+03$ \\
\hline Manganese -ICP-Fusion & $\mu \mathrm{g} / \mathrm{g}$ & $4.56 e+03$ & $4.67 \mathrm{e}+03$ \\
\hline Molybdenum -ICP-Fusion & $\mu \mathrm{g} / \mathrm{g}$ & $<9.63 \mathrm{e}+02$ & $<1.02 \mathrm{e}+03$ \\
\hline Sodium -ICP-Fusion & $\mu \mathrm{g} / \mathrm{g}$ & $2.42 e+04$ & $2.79 e+04$ \\
\hline Neodymium -ICP-Fusion & $\mu \mathrm{g} / \mathrm{g}$ & $<1.93 \mathrm{e}+03$ & $<2.04 \mathrm{e}+03$ \\
\hline Phosphorus-ICP-Fusion & $\mu \mathrm{g} / \mathrm{g}$ & $<3.85 \mathrm{e}+03$ & $<4.07 \mathrm{e}+03$ \\
\hline Lead-ICP-Fusion & $\mu \mathrm{g} / \mathrm{g}$ & $<1.93 \mathrm{e}+03$ & $<2.04 \mathrm{e}+03$ \\
\hline Sulfur-ICP-Fusion & $\mu \mathrm{g} / \mathrm{g}$ & $<1.93 \mathrm{e}+03$ & $<2.04 \mathrm{e}+03$ \\
\hline Antimony -ICP-Fusion & $\mu \mathrm{g} / \mathrm{g}$ & $<1.16 \mathrm{e}+03$ & $<1.22 \mathrm{e}+03$ \\
\hline Selenium -ICP-Fusion & $\mu \mathrm{g} / \mathrm{g}$ & $<1.93 \mathrm{e}+03$ & $<2.04 \mathrm{e}+03$ \\
\hline Silicon-ICP-Fusion & $\mu \mathrm{g} / \mathrm{g}$ & $9.07 e+03$ & $8.17 e+03$ \\
\hline Samarium-ICP-Fusion & $\mu \mathrm{g} / \mathrm{g}$ & $<1.93 \mathrm{e}+03$ & $<2.04 \mathrm{e}+03$ \\
\hline Strontium -ICP-Fusion & $\mu \mathrm{g} / \mathrm{g}$ & $<1.93 \mathrm{e}+02$ & $<2.04 \mathrm{e}+02$ \\
\hline Titanium-ICP-Fusion & $\mu \mathrm{g} / \mathrm{g}$ & $2.68 \mathrm{e}+02$ & $2.62 \theta+02$ \\
\hline Thallium -ICP-Fusion & $\mu \mathrm{g} / \mathrm{g}$ & $<3.85 \mathrm{e}+03$ & $<4.07 \mathrm{e}+03$ \\
\hline Uranium -ICP-Fusion & $\mu \mathrm{g} / \mathrm{g}$ & $2.20 \mathrm{e}+04$ & $2.17 \mathrm{e}+04$ \\
\hline Vanadium-ICP-Fusion & $\mu \mathrm{g} / \mathrm{g}$ & $<9.63 e+02$ & $<1.02 \mathrm{e}+03$ \\
\hline Zinc -ICP-Fusion & $\mu \mathrm{g} / \mathrm{g}$ & $<1.93 \mathrm{e}+02$ & $<2.04 \mathrm{e}+02$ \\
\hline Zirconium -ICP-Fusion & $\mu \mathrm{g} / \mathrm{g}$ & $2.42 e+04$ & $2.49 \mathrm{e}+04$ \\
\hline
\end{tabular}

(continued on next page) 
Table 3-10 Composition of Target Dilution Centrifuged Solids (page 2 of 3)

\begin{tabular}{|c|c|c|c|}
\hline Silver -ICP-Acid Digest & $\mu \mathrm{g} / \mathrm{g}$ & $5.41 \mathrm{e}+02$ & $4.84 \mathrm{e}+02$ \\
\hline Aluminium-ICP-Acid Digest & $\mu \mathrm{g} / \mathrm{g}$ & $9.72 e+04$ & $1.06 \mathrm{e}+05$ \\
\hline Arsenic -ICP-Acid Digest & $\mu \mathrm{g} / \mathrm{g}$ & $<3.89 \mathrm{e}+02$ & 77.5 \\
\hline Boron -ICP-Acid Digest & $\mu \mathrm{g} / \mathrm{g}$ & 57.6 & $<18.4$ \\
\hline Barium -ICP-Acid Digest & $\mu \mathrm{g} / \mathrm{g}$ & 94.0 & 71.8 \\
\hline Beryllium-ICP-Acid Digest & $\mu \mathrm{g} / \mathrm{g}$ & 24.8 & 18.6 \\
\hline Bismuth -ICP-Acid Digest & $\mu \mathrm{g} / \mathrm{g}$ & $6.61 \mathrm{e}+03$ & $4.87 e+03$ \\
\hline Calcium -ICP-Acid Digest & $\mu \mathrm{g} / \mathrm{g}$ & $3.03 e+03$ & $2.56 \mathrm{e}+03$ \\
\hline Cadmium -ICP-Acid Digest & $\mu \mathrm{g} / \mathrm{g}$ & $4.09 e+02$ & $2.98 \mathrm{e}+02$ \\
\hline Cerium -ICP-Acid Digest & $\mu \mathrm{g} / \mathrm{g}$ & $<38.9$ & $<36.8$ \\
\hline Cobalt -ICP-Acid Digest & $\mu \mathrm{g} / \mathrm{g}$ & $<7.78$ & $<7.35$ \\
\hline Chromium -ICP-Acid Digest & $\mu \mathrm{g} / \mathrm{g}$ & $8.71 \mathrm{e}+02$ & $6.42 \mathrm{e}+02$ \\
\hline Copper-ICP-Acid Digest & $\mu \mathrm{g} / \mathrm{g}$ & 54.1 & 42.8 \\
\hline Iron -ICP-Acid Digest & $\mu \mathrm{g} / \mathrm{g}$ & $1.75 \mathrm{e}+04$ & $1.30 \mathrm{e}+04$ \\
\hline Potassium -ICP-Acid Digest & $\mu \mathrm{g} / \mathrm{g}$ & $<1.95 \mathrm{e}+02$ & $<1.84 \mathrm{e}+02$ \\
\hline Lanthanum -ICP-Acid Digest & $\mu \mathrm{g} / \mathrm{g}$ & $<1.95 \mathrm{e}+02$ & $<18.4$ \\
\hline Lithium -ICP-Acid Digest & $\mu \mathrm{g} / \mathrm{g}$ & 8.53 & 5.99 \\
\hline Magnesium -ICP-Acid Digest & $\mu \mathrm{g} / \mathrm{g}$ & $8.17 e+02$ & $6.16 e+02$ \\
\hline Manganese -ICP-Acid Digest & $\mu \mathrm{g} / \mathrm{g}$ & $5.31 \mathrm{e}+03$ & $3.88 \mathrm{e}+03$ \\
\hline Molybdenum -ICP-Acid Digest & $\mu \mathrm{g} / \mathrm{g}$ & $<19.50$ & $<18.40$ \\
\hline Sodium -ICP-Acid Digest & $\mu \mathrm{g} / \mathrm{g}$ & $2.70 \mathrm{e}+04$ & $2.30 \mathrm{e}+04$ \\
\hline Neodymium -ICP-Acid Digest & $\mu \mathrm{g} / \mathrm{g}$ & $3.66 \mathrm{e}+02$ & $2.76 \mathrm{e}+02$ \\
\hline Nickel -ICP-Acid Digest & $\mu \mathrm{g} / \mathrm{g}$ & $1.36 \mathrm{e}+03$ & $9.81 \mathrm{e}+02$ \\
\hline Phosphorus -ICP-Acid Digest & $\mu \mathrm{g} / \mathrm{g}$ & $<7.78 \mathrm{e}+02$ & $6.78 \mathrm{e}+02$ \\
\hline Lead -ICP-Acid Digest & $\mu \mathrm{g} / \mathrm{g}$ & $7.77 \mathrm{e}+02$ & $5.91 \mathrm{e}+02$ \\
\hline Sulfur-ICP-Acid Digest & $\mu \mathrm{g} / \mathrm{g}$ & $1.70 \mathrm{e}+02$ & $1.30 \mathrm{e}+02$ \\
\hline Antimony -ICP-Acid Digest & $\mu \mathrm{g} / \mathrm{g}$ & $<23.3$ & $<22.1$ \\
\hline Selenium -ICP-Acid Digest & $\mu \mathrm{g} / \mathrm{g}$ & $<38.9$ & $<36.8$ \\
\hline Silicon -ICP-Acid Digest & $\mu \mathrm{g} / \mathrm{g}$ & $1.25 \mathrm{e}+03$ & $7.73 e+02$ \\
\hline Samarium-ICP-Acid Digest & $\mu \mathrm{g} / \mathrm{g}$ & $<38.9$ & $<36.8$ \\
\hline Strontium -ICP-Acid Digest & $\mu \mathrm{g} / \mathrm{g}$ & 82.5 & 70.4 \\
\hline Titanium-ICP-Acid Digest & $\mu \mathrm{g} / \mathrm{g}$ & $2.46 \mathrm{e}+02$ & $1.89 \mathrm{e}+02$ \\
\hline Thallium -ICP-Acid Digest & $\mu \mathrm{g} / \mathrm{g}$ & $<77.8$ & $<73.5$ \\
\hline Uranium -ICP-Acid Digest & $\mu \mathrm{g} / \mathrm{g}$ & $2.58 \mathrm{e}+04$ & $1.95 e+04$ \\
\hline Vanadium -ICP-Acid Digest & $\mu \mathrm{g} / \mathrm{g}$ & $<19.5$ & $<18.4$ \\
\hline Zinc-ICP-Acid Digest & $\mu \mathrm{g} / \mathrm{g}$ & 80.6 & 64.1 \\
\hline Zirconium -ICP-Acid Digest & $\mu \mathrm{g} / \mathrm{g}$ & $2.71 \mathrm{e}+04$ & $1.91 \mathrm{e}+04$ \\
\hline Mercury & $\mu \mathrm{g} / \mathrm{g}$ & 15.0 & 13.8 \\
\hline Cyanide & $\mu \mathrm{g} / \mathrm{g}$ & $<10.8$ & $<10.6$ \\
\hline Ammonia & $\mu \mathrm{g} / \mathrm{g}$ & $<9.84 \mathrm{e}+03$ & $8.42 \mathrm{e}+03$ \\
\hline
\end{tabular}

(continued on next page) 
Table 3-10 Composition of Target Dilution Centrifuged Solids (page 3 of 3)

\begin{tabular}{|l|c|c|c|}
\hline Fluoride & $\mu \mathrm{g} / \mathrm{g}$ & $8.12 \mathrm{e}+03$ & $7.43 \mathrm{e}+03$ \\
\hline Chloride & $\mu \mathrm{g} / \mathrm{g}$ & $1.15 \mathrm{e}+02$ & 90.5 \\
\hline Nitrite & $\mu \mathrm{g} / \mathrm{g}$ & $2.94 \mathrm{e}+03$ & $2.66 \mathrm{e}+03$ \\
\hline Bromide & $\mu \mathrm{g} / \mathrm{g}$ & $<2.71 \mathrm{e}+02$ & $<2.66 \mathrm{e}+02$ \\
\hline Nitrate & $\mu \mathrm{g} / \mathrm{g}$ & $2.74 \mathrm{e}+03$ & $2.54 \mathrm{e}+03$ \\
\hline Phosphate & $\mu \mathrm{g} / \mathrm{g}$ & $2.39 \mathrm{e}+03$ & $2.34 \mathrm{e}+03$ \\
\hline Sulfate & $\mu \mathrm{g} / \mathrm{g}$ & $5.10 \mathrm{e}+02$ & $4.01 \mathrm{e}+02$ \\
\hline Oxalate & $\mu \mathrm{g} / \mathrm{g}$ & $1.08 \mathrm{e}+03$ & $9.81 \mathrm{e}+02$ \\
\hline Alpha & $\mu \mathrm{Ci} / \mathrm{g}$ & 4.73 & 4.55 \\
\hline Beta & $\mu \mathrm{Ci} / \mathrm{g}$ & $7.14 \mathrm{e}+02$ & $6.63 \mathrm{e}+02$ \\
\hline Tritium & $\mu \mathrm{Ci} / \mathrm{g}$ & $<5.93 \mathrm{e}-04$ & $7.76 \mathrm{e}-03$ \\
\hline C-14 & $\mu \mathrm{Ci} / \mathrm{g}$ & $<4.02 \mathrm{e}-04$ & $<3.95 \mathrm{e}-04$ \\
\hline Cobalt-60 & $\mu \mathrm{Ci} / \mathrm{g}$ & $1.24 \mathrm{e}-01$ & $8.84 \mathrm{e}-02$ \\
\hline Strontium-89/90 & $\mu \mathrm{Ci} / \mathrm{g}$ & $3.43 \mathrm{e}+02$ & $3.16 \mathrm{e}+02$ \\
\hline Technetium-99 by ICP/MS & $\mu \mathrm{Ci} / \mathrm{g}$ & $<2.78 \mathrm{e}-02$ & $<2.63 \mathrm{e}-02$ \\
\hline Technetium-99 & $\mu \mathrm{Ci} / \mathrm{g}$ & $1.74 \mathrm{e}-02$ & $5.83 \mathrm{e}-02$ \\
Liquid Scintillation & & & \\
\hline Antimony-125 & $\mu \mathrm{Ci} / \mathrm{g}$ & $<2.59 \mathrm{e}-01$ & $<2.57 \mathrm{e}-01$ \\
\hline Tin-126 & $\mu \mathrm{g} / \mathrm{g}$ & $<1.23 \mathrm{e}+02$ & $<1.16 \mathrm{e}+02$ \\
\hline Iodine-129 & $\mu \mathrm{Ci} / \mathrm{g}$ & $<5.97 \mathrm{e}-02$ & $<4.93 \mathrm{e}-02$ \\
\hline Cesium-137 & $\mu \mathrm{Ci} / \mathrm{g}$ & 37.2 & 33.4 \\
\hline Europium-152 & $\mu \mathrm{Ci} / \mathrm{g}$ & $<1.58 \mathrm{e}-01$ & $<1.57 \mathrm{e}-01$ \\
\hline Europium-154 & $\mu \mathrm{Ci} / \mathrm{g}$ & $3.08 \mathrm{e}-01$ & $3.96 \mathrm{e}-01$ \\
\hline Europium-155 & $\mu \mathrm{Ci} / \mathrm{g}$ & $<1.96 \mathrm{e}-01$ & $<1.97 \mathrm{e}-01$ \\
\hline Americium-241 & $\mu \mathrm{Ci} / \mathrm{g}$ & 1.7 & 1.66 \\
\hline Curium-243/244 & $\mu \mathrm{Ci} / \mathrm{g}$ & $<2.21 \mathrm{e}-01$ & $<2.29 \mathrm{e}-01$ \\
\hline Uranium-233 & $\mu \mathrm{g} / \mathrm{g}$ & 53.5 & 37.9 \\
\hline Uranium-234 & $\mu \mathrm{g} / \mathrm{g}$ & $<9.85$ & $<9.31$ \\
\hline Uranium-235 & $\mu \mathrm{g} / \mathrm{g}$ & $1.93 \mathrm{e}+02$ & $1.51 \mathrm{e}+02$ \\
\hline Uranium-236 & $\mu \mathrm{g} / \mathrm{g}$ & 13.2 & $<12.4$ \\
\hline Uranium-238 & $\mu \mathrm{g} / \mathrm{g}$ & $2.74 \mathrm{e}+04$ & $2.23 \mathrm{e}+04$ \\
\hline Neptunium-237 & $\mu \mathrm{g} / \mathrm{g}$ & $<11.9$ & $<11.2$ \\
\hline Plutonium-239 & $\mu \mathrm{g} / \mathrm{g}$ & 37.5 & 28.8 \\
\hline Plutonium-240 & $\mu \mathrm{g} / \mathrm{g}$ & $<15.0$ & $<14.2$ \\
\hline Plutonium-242 & $\mu \mathrm{g} / \mathrm{g}$ & $<12.6$ & $<11.9$ \\
\hline Pu/Am-241 & $\mu \mathrm{g} / \mathrm{g}$ & $<11.2$ & $<10.6$ \\
\hline Am 243/Cm 243 & $\mu \mathrm{g} / \mathrm{g}$ & $<10.8$ & $<10.2$ \\
\hline Pu 244/Cm 244 & $\mu \mathrm{g} / \mathrm{g}$ & $<11.2$ & $<10.5$ \\
\hline Mass Balance & $\mathrm{wt} \%$ & 97.2 & 89.5 \\
\hline Charge Balance & -- & 0.94 & 1.14 \\
\hline & & & \\
\hline
\end{tabular}


RPP-5798, Rev. 0

Table 3-11 Comparison of Fusion and Adid Digest for ICP/AES Analyses

\begin{tabular}{|c|c|c|c|c|c|}
\hline \multirow[t]{2}{*}{ Analyte } & \multirow[t]{2}{*}{ Unit } & \multicolumn{2}{|c|}{ TD Solids } & \multicolumn{2}{|c|}{ TD Solids (Duplicate) } \\
\hline & & ICP Fusion & Acid Digest & ICP Fusion & Acid Digest \\
\hline Silver & $\mu \mathrm{g} / \mathrm{g}$ & $6.16 e+02$ & $5.41 \mathrm{e}+02$ & $5.17 \mathrm{e}+02$ & $4.84 \mathrm{e}+02$ \\
\hline Aluminium & $\mu \mathrm{g} / \mathrm{g}$ & $1.16 \mathrm{e}+05$ & $9.72 \mathrm{e}+04$ & $9.80 \mathrm{e}+04$ & $1.06 e+05$ \\
\hline Arsenic & $\mu \mathrm{g} / \mathrm{g}$ & $<1.93 \mathrm{e}+03$ & $<3.89 \mathrm{e}+02$ & $<2.04 \mathrm{e}+03$ & 77.5 \\
\hline Boron & $\mu \mathrm{g} / \mathrm{g}$ & $<9.63 \mathrm{e}+02$ & 57.6 & $<1.02 \mathrm{e}+03$ & $<18.4$ \\
\hline Barium & $\mu \mathrm{g} / \mathrm{g}$ & $<9.63 \mathrm{e}+02$ & 94.0 & $<1.02 \mathrm{e}+03$ & 71.8 \\
\hline Beryllium & $\mu \mathrm{g} / \mathrm{g}$ & $<96.30$ & 24.8 & $<1.02 \mathrm{e}+02$ & 18.6 \\
\hline Bismuth & $\mu \mathrm{g} / \mathrm{g}$ & $6.16 \mathrm{e}+03$ & $6.61 \mathrm{e}+03$ & $5.82 \mathrm{e}+03$ & $4.87 \mathrm{e}+03$ \\
\hline Calcium & $\mu \mathrm{g} / \mathrm{g}$ & $3.30 \mathrm{e}+03$ & $3.03 \mathrm{e}+03$ & $2.79 e+03$ & $2.56 \mathrm{e}+03$ \\
\hline Cadmium & $\mu \mathrm{g} / \mathrm{g}$ & $3.86 \mathrm{e}+02$ & $4.09 \mathrm{e}+02$ & $3.99 \mathrm{e}+02$ & $2.98 \mathrm{e}+02$ \\
\hline Cerium & $\mu \mathrm{g} / \mathrm{g}$ & $<1.93 \mathrm{e}+03$ & $<38.9$ & $<2.04 \mathrm{e}+03$ & $<36.8$ \\
\hline Cobalt & $\mu \mathrm{g} / \mathrm{g}$ & $<3.85 \mathrm{e}+02$ & $<7.78$ & $<4.07 e+02$ & $<7.35$ \\
\hline Chromium & $\mu \mathrm{g} / \mathrm{g}$ & $8.48 \mathrm{e}+02$ & $8.71 \mathrm{e}+02$ & $8.66 \mathrm{e}+02$ & $6.42 \mathrm{e}+02$ \\
\hline Copper & $\mu \mathrm{g} / \mathrm{g}$ & $<1.93 e+02$ & 54.1 & $<2.04 \mathrm{e}+02$ & 42.8 \\
\hline Iron & $\mu \mathrm{g} / \mathrm{g}$ & $1.64 \mathrm{e}+04$ & $1.75 \mathrm{e}+04$ & $1.66 \mathrm{e}+04$ & $1.30 \mathrm{e}+04$ \\
\hline Potassium & $\mu \mathrm{g} / \mathrm{g}$ & Not Reported & $<1.95 \mathrm{e}+02$ & Not Reported & $<1.84 \mathrm{e}+02$ \\
\hline Lanthanum & $\mu \mathrm{g} / \mathrm{g}$ & $<9.63 \mathrm{e}+02$ & $<1.95 \mathrm{e}+02$ & $<1.02 \mathrm{e}+03$ & $<18.4$ \\
\hline Lithium & $\mu \mathrm{g} / \mathrm{g}$ & $<1.93 \mathrm{e}+02$ & 8.53 & $<2.04 \mathrm{e}+02$ & 5.99 \\
\hline Magnesium & $\mu \mathrm{g} / \mathrm{g}$ & $<1.93 \mathrm{e}+03$ & $8.17 \mathrm{e}+02$ & $<2.04 \mathrm{e}+03$ & $6.16 \mathrm{e}+02$ \\
\hline Manganese & $\mu \mathrm{g} / \mathrm{g}$ & $4.56 \mathrm{e}+03$ & $5.31 \mathrm{e}+03$ & $4.67 \mathrm{e}+03$ & $3.88 \mathrm{e}+03$ \\
\hline Molybdenum & $\mu \mathrm{g} / \mathrm{g}$ & $<9.63 \mathrm{e}+02$ & $<19.50$ & $<1.02 \mathrm{e}+03$ & $<18.40$ \\
\hline Sodium & $\mu \mathrm{g} / \mathrm{g}$ & $2.42 \mathrm{e}+04$ & $2.70 \mathrm{e}+04$ & $2.79 \mathrm{e}+04$ & $2.30 \mathrm{e}+04$ \\
\hline Neodymium & $\mu \mathrm{g} / \mathrm{g}$ & $<1.93 \mathrm{e}+03$ & $3.66 \mathrm{e}+02$ & $<2.04 \mathrm{e}+03$ & $2.76 \mathrm{e}+02$ \\
\hline Nickel & $\mu \mathrm{g} / \mathrm{g}$ & Not Reported & $1.36 \mathrm{e}+03$ & Not Reported & $9.81 \mathrm{e}+02$ \\
\hline Phosphorus & $\mu \mathrm{g} / \mathrm{g}$ & $<3.85 \mathrm{e}+03$ & $<7.78 \mathrm{e}+02$ & $<4.07 \mathrm{e}+03$ & $6.78 \mathrm{e}+02$ \\
\hline Lead & $\mu \mathrm{g} / \mathrm{g}$ & $<1.93 \mathrm{e}+03$ & $7.77 e+02$ & $<2.04 \mathrm{e}+03$ & $5.91 \mathrm{e}+02$ \\
\hline Sulfur & $\mu \mathrm{g} / \mathrm{g}$ & $<1.93 \mathrm{e}+03$ & $1.70 e+02$ & $<2.04 e+03$ & $1.30 \mathrm{e}+02$ \\
\hline Antimony & $\mu \mathrm{g} / \mathrm{g}$ & $<1.16 \mathrm{e}+03$ & $<23.3$ & $<1.22 \mathrm{e}+03$ & $<22.1$ \\
\hline Selenium & $\mu \mathrm{g} / \mathrm{g}$ & $<1.93 \mathrm{e}+03$ & $<38.9$ & $<2.04 \mathrm{e}+03$ & $<36.8$ \\
\hline Silicon & $\mu \mathrm{g} / \mathrm{g}$ & $9.07 e+03$ & $1.25 \mathrm{e}+03$ & $8.17 \mathrm{e}+03$ & $7.73 e+02$ \\
\hline Samarium & $\mu \mathrm{g} / \mathrm{g}$ & $<1.93 \mathrm{e}+03$ & $<38.9$ & $<2.04 \mathrm{e}+03$ & $<36.8$ \\
\hline Strontium & $\mu \mathrm{g} / \mathrm{g}$ & $<1.93 \mathrm{e}+02$ & 82.5 & $<2.04 \mathrm{e}+02$ & 70.4 \\
\hline Titanium & $\mu \mathrm{g} / \mathrm{g}$ & $2.68 \mathrm{e}+02$ & $2.46 \mathrm{e}+02$ & $2.62 \mathrm{e}+02$ & $1.89 \mathrm{e}+02$ \\
\hline Thallium & $\mu \mathrm{g} / \mathrm{g}$ & $<3.85 e+03$ & $<77.8$ & $<4.07 e+03$ & $<73.5$ \\
\hline Uranium & $\mu \mathrm{g} / \mathrm{g}$ & $2.20 \mathrm{e}+04$ & $2.58 \mathrm{e}+04$ & $2.17 \mathrm{e}+04$ & $1.95 \mathrm{e}+04$ \\
\hline$\overline{\mathrm{V}}$ anadium & $\mu \mathrm{g} / \mathrm{g}$ & $<9.63 \mathrm{e}+02$ & $<19.5$ & $<1.02 \mathrm{e}+03$ & $<18.4$ \\
\hline Zinc & $\mu \mathrm{g} / \mathrm{g}$ & $<1.93 \mathrm{e}+02$ & 80.6 & $<2.04 \mathrm{e}+02$ & 64.1 \\
\hline Zirconium & $\mu \mathrm{g} / \mathrm{g}$ & $2.42 \mathrm{e}+04$ & $2.71 \mathrm{e}+04$ & $2.49 \mathrm{e}+04$ & $1.91 \mathrm{e}+04$ \\
\hline
\end{tabular}


RPP-5798, Rev. 0

\subsection{SLUDGE COMPOSITION AS A FUNCTION OF SETTLING}

Duplicate samples of the target dilution slurry were divided into three fractions differentiated by the settling rate of the solids. The procedure used to separate the samples into the three fractions was discussed in Section 2.6. Each fraction was centrifuged and the centrifuged solids were submitted for the analyses shown in Table 3-12.

The middle third duplicate sample in Table 3-12 had a percent water result significantly lower than the other TGA results reported throughout this study. This value should be considered highly questionable.

Figures 3-4 through 3-6 are graphical representations of selected data from Table 3-12. To make comparisons of different analytes simpler, all of the analytical results were divided by the result for the top sample (i.e., normalized).

A few trends are evident in Figures 3-4 through 3-6. The aluminum concentrations were greater in both samples collected from the middle third fractions. Most of the other analytes had a corresponding decrease in their concentrations in the middle third fractions. Additionally, the bottom third samples had alpha and beta levels significantly higher than the other two fractions, but not dramatically so. 
Table 3-12 Sludge Composition as a Function of Sludge Settling (page 1 of 2)

\begin{tabular}{|c|c|c|c|c|c|c|c|}
\hline Analyte & Unit & Top Third & \begin{tabular}{|c|} 
Top Third \\
(Duplicate)
\end{tabular} & $\begin{array}{l}\text { Middle } \\
\text { Third }\end{array}$ & \begin{tabular}{|c|} 
Middle \\
Third \\
(Duplicate)
\end{tabular} & $\begin{array}{c}\text { Bottom } \\
\text { Third }\end{array}$ & $\begin{array}{c}\text { Bottom } \\
\text { Third } \\
\text { (Duplicate) }\end{array}$ \\
\hline$\%$ Water by TGA & $\%$ & 54.76 & 56.61 & 55.54 & $(40.80)$ & 51.36 & 55.35 \\
\hline Silver & $\mu \mathrm{g} / \mathrm{g}$ & $5.19 e+02$ & $5.17 \mathrm{e}+02$ & $5.31 \mathrm{e}+02$ & $5.38 \mathrm{e}+02$ & $5.87 e+02$ & $4.55 e+02$ \\
\hline Aluminium & $\mu \mathrm{g} / \mathrm{g}$ & $5.34 \mathrm{e}+04$ & $1.12 \mathrm{e}+05$ & $1.04 e+05$ & $1.41 \mathrm{e}+05$ & $8.77 \mathrm{e}+04$ & $8.19 e+04$ \\
\hline Arsenic & $\mu \mathrm{g} / \mathrm{g}$ & $<2.59 \mathrm{e}+03$ & $<2.43 \mathrm{e}+03$ & $<2.54 \mathrm{e}+03$ & $<2.38 \mathrm{e}+03$ & $<2.53 \mathrm{e}+03$ & $<2.53 \mathrm{e}+03$ \\
\hline Boron & $\mu \mathrm{g} / \mathrm{g}$ & $<1.30 \mathrm{e}+03$ & $<1.22 \mathrm{e}+03$ & $<1.27 \mathrm{e}+03$ & $<1.19 \mathrm{e}+03$ & $<1.26 \mathrm{e}+03$ & $<1.27 \mathrm{e}+03$ \\
\hline Barium & $\mu \mathrm{g} / \mathrm{g}$ & $<1.30 \mathrm{e}+03$ & $<1.22 \mathrm{e}+03$ & $<1.27 \mathrm{e}+03$ & $<1.19 \mathrm{e}+03$ & $<1.26 \mathrm{e}+03$ & $<1.27 \mathrm{e}+03$ \\
\hline Beryllium & $\mu \mathrm{g} / \mathrm{g}$ & $<1.30 \mathrm{e}+02$ & $<1.22 \mathrm{e}+02$ & $<1.27 \mathrm{e}+02$ & $<1.19 \mathrm{e}+02$ & $<1.26 \mathrm{e}+02$ & $<1.27 \mathrm{e}+02$ \\
\hline Bismuth & $\mu \mathrm{g} / \mathrm{g}$ & $<2.59 \mathrm{e}+03$ & $<2.43 \mathrm{e}+03$ & $<2.54 \mathrm{e}+03$ & $<2.38 \mathrm{e}+03$ & $<2.53 \mathrm{e}+03$ & $<2.53 e+03$ \\
\hline Calcium & $\mu \mathrm{g} / \mathrm{g}$ & $4.91 \mathrm{e}+03$ & $3.77 e+03$ & $3.93 e+03$ & $3.46 e+03$ & $5.42 \mathrm{e}+03$ & $4.16 \mathrm{e}+03$ \\
\hline Cadmium & $\mu g / g$ & $3.00 \mathrm{e}+02$ & $4.95 \mathrm{e}+02$ & $3.10 \mathrm{e}+02$ & $4.30 \mathrm{e}+02$ & $2.39 e+02$ & $3.05 e+02$ \\
\hline Cerium & $\mu \mathrm{g} / \mathrm{g}$ & $<2.59 \mathrm{e}+03$ & $<2.43 e+03$ & $<2.54 \mathrm{e}+03$ & $<2.38 \mathrm{e}+03$ & $<2.53 \mathrm{e}+03$ & $<2.53 \mathrm{e}+03$ \\
\hline Cobalt & $\mu \mathrm{g} / \mathrm{g}$ & $<5.19 \mathrm{e}+02$ & $<4.87 e+02$ & $<5.08 \mathrm{e}+02$ & $<4.75 \mathrm{e}+02$ & $<5.06 \mathrm{e}+02$ & $<5.07 e+02$ \\
\hline Chromium & $\mu \mathrm{g} / \mathrm{g}$ & $1.09 \mathrm{e}+03$ & $8.72 e+02$ & $5.68 \mathrm{e}+02$ & $4.64 e+02$ & $1.16 e+03$ & $9.85 \mathrm{e}+02$ \\
\hline Copper & $\mu \mathrm{g} / \mathrm{g}$ & $<2.59 \mathrm{e}+02$ & $<2.43 \mathrm{e}+02$ & $<2.54 \mathrm{e}+02$ & $<2.38 \mathrm{e}+02$ & $<2.53 e+02$ & $<2.53 \mathrm{e}+02$ \\
\hline Iron & $\mu \mathrm{g} / \mathrm{g}$ & $2.22 \mathrm{e}+04$ & $1.48 \mathrm{e}+04$ & $1.47 \mathrm{e}+04$ & $1.28 \mathrm{e}+04$ & $2.13 e+04$ & $1.53 e+04$ \\
\hline Lanthanum & $\mu \mathrm{g} / \mathrm{g}$ & $<1.30 \mathrm{e}+03$ & $<1.22 \mathrm{e}+03$ & $<1.27 \mathrm{e}+03$ & $1.19 \mathrm{e}+03$ & $<1.26 \mathrm{e}+03$ & $<1.27 \mathrm{e}+03$ \\
\hline Lithium & $\mu \mathrm{g} / \mathrm{g}$ & $<2.59 \mathrm{e}+02$ & $<2.43 \mathrm{e}+02$ & $<2.54 \mathrm{e}+02$ & $<2.38 \mathrm{e}+02$ & $<2.53 \mathrm{e}+02$ & $<2.53 \mathrm{e}+02$ \\
\hline Magnesium & $\mu \mathrm{g} / \mathrm{g}$ & $<2.59 \mathrm{e}+03$ & $<2.43 \mathrm{e}+03$ & $<2.54 \mathrm{e}+03$ & $<2.38 \mathrm{e}+03$ & $<2.53 \mathrm{e}+03$ & $<2.53 \mathrm{e}+03$ \\
\hline Manganese & $\mu \mathrm{g} / \mathrm{g}$ & $6.96 \mathrm{e}+03$ & $4.53 \mathrm{e}+03$ & $4.61 \mathrm{e}+03$ & $3.85 e+03$ & $6.32 \mathrm{e}+03$ & $4.44 e+03$ \\
\hline Molybdenum & $\mu \mathrm{g} / \mathrm{g}$ & $<1.30 \mathrm{e}+03$ & $<1.22 \mathrm{e}+03$ & $<1.27 e+03$ & $<1.19 \mathrm{e}+03$ & $<1.26 \mathrm{e}+03$ & $<1.27 \mathrm{e}+03$ \\
\hline Sodium & $\mu \mathrm{g} / \mathrm{g}$ & $3.51 \mathrm{e}+04$ & $2.84 \mathrm{e}+04$ & $2.96 \mathrm{e}+04$ & $2.85 \mathrm{e}+04$ & $3.62 e+04$ & $3.01 \mathrm{e}+04$ \\
\hline Neodymium & $\mu \mathrm{g} / \mathrm{g}$ & $<2.59 \mathrm{e}+03$ & $<2.43 \mathrm{e}+03$ & $<2.54 \mathrm{e}+03$ & $<2.38 \mathrm{e}+03$ & $<2.53 \mathrm{e}+03$ & $<2.53 \mathrm{e}+03$ \\
\hline Phosphorus & $\mu \mathrm{g} / \mathrm{g}$ & $<5.19 \mathrm{e}+03$ & $<4.87 \mathrm{e}+03$ & $<5.08 \mathrm{e}+03$ & $<4.75 \mathrm{e}+03$ & $<5.06 \mathrm{e}+03$ & $<5.07 \mathrm{e}+03$ \\
\hline Lead & $\mu \mathrm{g} / \mathrm{g}$ & $<2.59 \mathrm{e}+03$ & $<2.43 \mathrm{e}+03$ & $<2.54 \mathrm{e}+03$ & $<2.38 \mathrm{e}+03$ & $<2.53 \mathrm{e}+03$ & $<2.53 \mathrm{e}+03$ \\
\hline Sulfur & $\mu \mathrm{g} / \mathrm{g}$ & $<2.59 \mathrm{e}+03$ & $<2.43 \mathrm{e}+03$ & $<2.54 \mathrm{e}+03$ & $<2.38 \mathrm{e}+03$ & $<2.5 \overline{3 e+03}$ & $<2.53 \mathrm{e}+03$ \\
\hline Antimony & $\mu \mathrm{g} / \mathrm{g}$ & $<1.56 \mathrm{e}+03$ & $<1.46 \mathrm{e}+03$ & $<1.52 \mathrm{e}+03$ & $<1.43 \mathrm{e}+03$ & $<1.52 \mathrm{e}+03$ & $<1.52 \mathrm{e}+03$ \\
\hline Selenium & $\mu \mathrm{g} / \mathrm{g}$ & $<2.59 \mathrm{e}+03$ & $<2.43 e+03$ & $<2.54 \mathrm{e}+03$ & $<2.38 \mathrm{e}+03$ & $<2.53 \mathrm{e}+03$ & $<2.53 \mathrm{e}+03$ \\
\hline Silicon & $\mu \mathrm{g} / \mathrm{g}$ & $7.04 \mathrm{e}+03$ & $8.21 \mathrm{e}+03$ & $8.54 \mathrm{e}+03$ & $8.70 \mathrm{e}+03$ & $9.39 \mathrm{e}+03$ & $7.00 e+03$ \\
\hline Samarium & $\mu \mathrm{g} / \mathrm{g}$ & $<2.59 \mathrm{e}+03$ & $<2.43 e+03$ & $<2.54 \mathrm{e}+03$ & $<2.38 \mathrm{e}+03$ & $<2.53 \mathrm{e}+03$ & $<2.53 \mathrm{e}+03$ \\
\hline Strontium & $\mu \mathrm{g} / \mathrm{g}$ & $<2.59 \mathrm{e}+02$ & $<2.43 \mathrm{e}+02$ & $<2.54 \mathrm{e}+02$ & $<2.38 \mathrm{e}+02$ & $<2.53 \mathrm{e}+02$ & $<2.53 \mathrm{e}+02$ \\
\hline Titanium & $\mu \mathrm{g} / \mathrm{g}$ & $<2.59 \mathrm{e}+02$ & $<2.43 \mathrm{e}+02$ & $<2.54 \mathrm{e}+02$ & $<2.38 \mathrm{e}+02$ & $<2.53 \mathrm{e}+02$ & $<2.53 \mathrm{e}+02$ \\
\hline Thallium & $\mu \mathrm{g} / \mathrm{g}$ & $<5.19 \mathrm{e}+03$ & $<4.87 e+03$ & $<5.08 \mathrm{e}+03$ & $<4.75 \mathrm{e}+03$ & $<5.06 \mathrm{e}+03$ & $<5.07 \mathrm{e}+03$ \\
\hline Uranium & $\mu \mathrm{g} / \mathrm{g}$ & $3.89 \mathrm{e}+04$ & $2.30 \mathrm{e}+04$ & $2.34 \mathrm{e}+04$ & $2.07 e+04$ & $3.52 \mathrm{e}+04$ & $2.54 \mathrm{e}+04$ \\
\hline Vanadium & $\mu \mathrm{g} / \mathrm{g}$ & $<1.30 \mathrm{e}+03$ & $<1.22 \mathrm{e}+03$ & $<1.27 e+03$ & $<1.19 \mathrm{e}+03$ & $<1.26 \mathrm{e}+03$ & $<1.27 \mathrm{e}+03$ \\
\hline Zinc & $\mu \mathrm{g} / \mathrm{g}$ & $2.70 \mathrm{e}+02$ & $3.24 \mathrm{e}+02$ & $2.96 \mathrm{e}+02$ & $2.64 e+02$ & $3.35 e+02$ & $<2.53 e+02$ \\
\hline Zirconium & $\mu \mathrm{g} / \mathrm{g}$ & $4.57 \mathrm{e}+04$ & $2.61 \mathrm{e}+04$ & $2.58 \mathrm{e}+04$ & $2.25 e+04$ & $4.08 \mathrm{e}+04$ & $2.90 \mathrm{e}+04$ \\
\hline
\end{tabular}


RPP-5798, Rev. 0

Table 3-12 Sludge Composition as a Function of Sludge Settling (page 2 of 2) -

\begin{tabular}{|l|c|c|c|c|c|c|c|}
\hline \multicolumn{1}{|c|}{ Analyte } & Unit & Top Third & $\begin{array}{c}\text { Top Third } \\
\text { (Duplicate) }\end{array}$ & $\begin{array}{c}\text { Middle } \\
\text { Third }\end{array}$ & $\begin{array}{c}\text { Middle } \\
\text { Third } \\
\text { (Duplicate) }\end{array}$ & $\begin{array}{c}\text { Bottom } \\
\text { Third }\end{array}$ & $\begin{array}{c}\text { Bottom } \\
\text { Third } \\
\text { (Duplicate) }\end{array}$ \\
\hline Alpha & $\mu \mathrm{Ci} / \mathrm{g}$ & 4.44 & 4.26 & 4.12 & 3.53 & 5.99 & 5.89 \\
\hline Beta & $\mu \mathrm{Ci} / \mathrm{g}$ & $6.57 \mathrm{e}+02$ & $6.49 \mathrm{e}+02$ & $6.05 \mathrm{e}+02$ & $5.48 \mathrm{e}+02$ & $8.54 \mathrm{e}+02$ & $8.58 \mathrm{e}+02$ \\
\hline Cobalt-60 & $\mu \mathrm{Ci} / \mathrm{g}$ & $<1.26 \mathrm{e}-01$ & $<2.34 \mathrm{e}-01$ & $<2.34 \mathrm{e}-01$ & $<2.18 \mathrm{e}-01$ & $<1.33 \mathrm{e}-01$ & $<1.06 \mathrm{e}-01$ \\
\hline Antimony-125 & $\mu \mathrm{Ci} / \mathrm{g}$ & $<5.32 \mathrm{e}-01$ & $<6.94 \mathrm{e}-01$ & $<7.14 \mathrm{e}-01$ & $<7.05 \mathrm{e}-01$ & $<5.88 \mathrm{e}-01$ & $<5.26 \mathrm{e}-01$ \\
\hline Cesium-137 & $\mu \mathrm{Ci} / \mathrm{g}$ & 31.5 & 31.9 & 33.8 & 35.1 & 39.4 & 29.2 \\
\hline Europium-152 & $\mu \mathrm{Ci} / \mathrm{g}$ & $<3.71 \mathrm{e}-01$ & $<4.17 \mathrm{e}-01$ & $<4.34 \mathrm{e}-01$ & $<4.19 \mathrm{e}-01$ & $<4.06 \mathrm{e}-01$ & $<3.99 \mathrm{e}-01$ \\
\hline Europium-154 & $\mu \mathrm{Ci} / \mathrm{g}$ & $<3.38 \mathrm{e}-01$ & $<6.68 \mathrm{e}-01$ & $<6.80 \mathrm{e}-01$ & $<6.55 \mathrm{e}-01$ & $5.16 \mathrm{e}-01$ & $5.51 \mathrm{e}-01$ \\
\hline Europium-155 & $\mu \mathrm{Ci} / \mathrm{g}$ & $<4.50 \mathrm{e}-01$ & $<5.45 \mathrm{e}-01$ & $<5.51 \mathrm{e}-01$ & $<5.23 \mathrm{e}-01$ & $<4.92 \mathrm{e}-01$ & $<4.74 \mathrm{e}-01$ \\
\hline
\end{tabular}


Figure 3-3 Normalized Concentrations of Selected Species as a Function of Settling

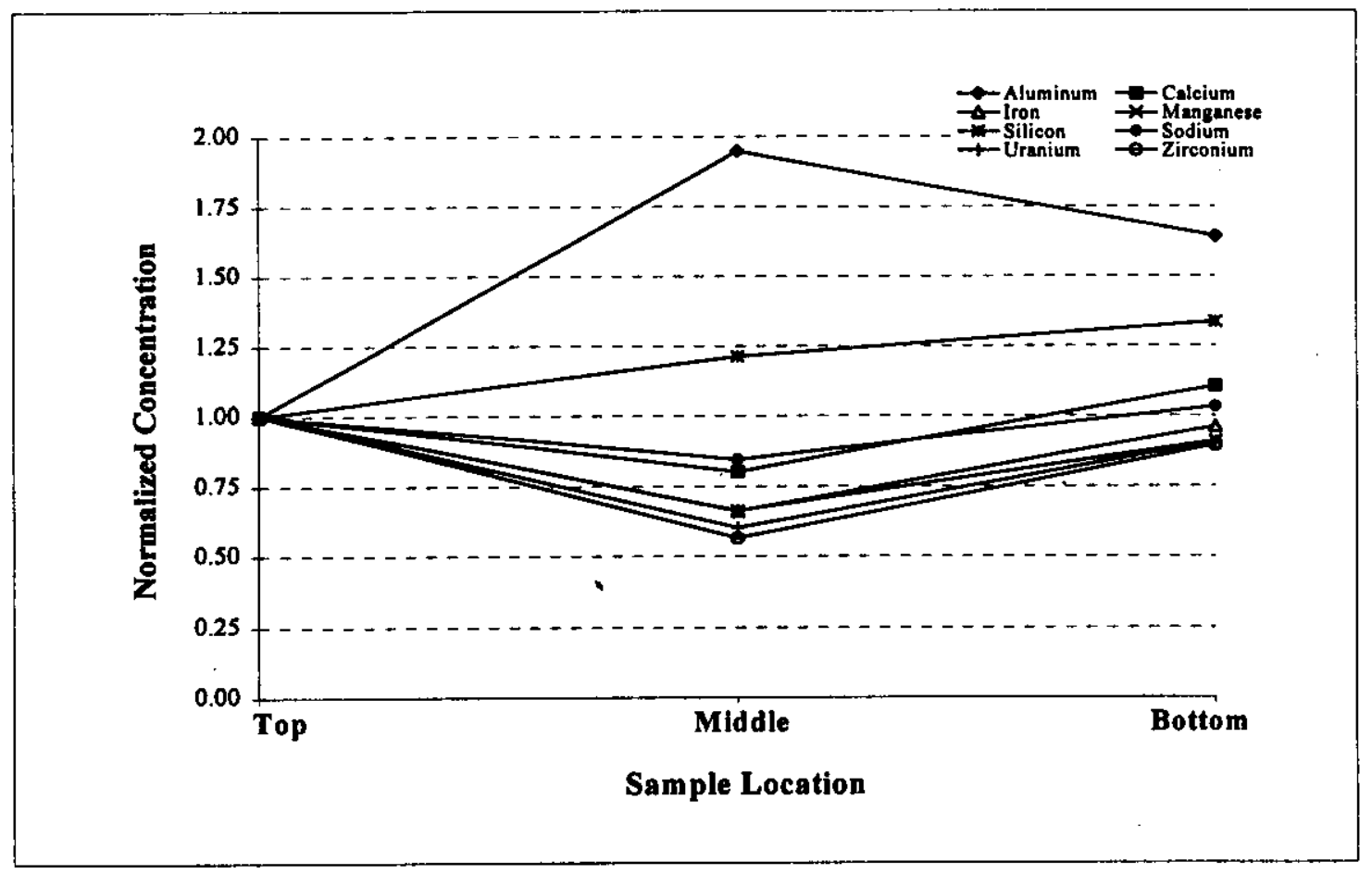

Figure 3-4 Normalized Concentrations of Selected Species as a Function of Settling (Duplicate Sample)

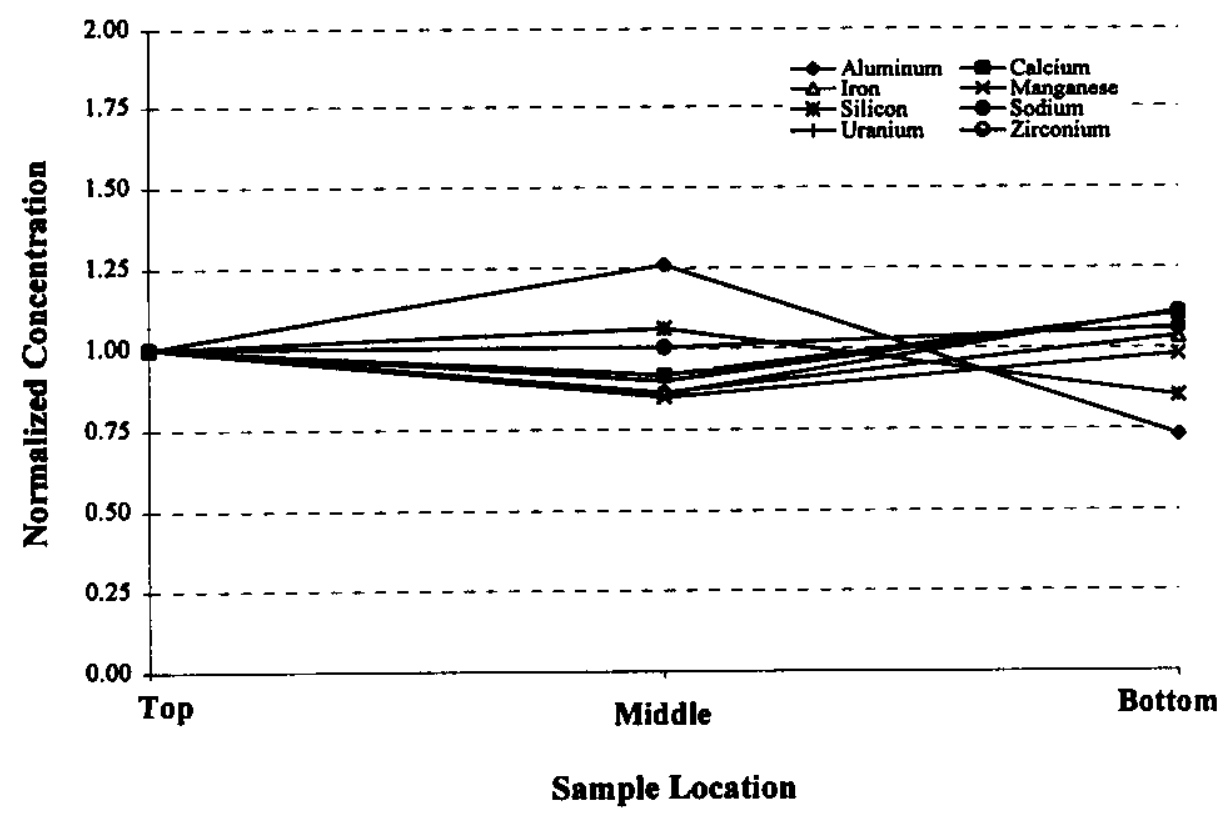


Figure 3-5 Normalized Radiochemical Concentrations as a Function of Settling -

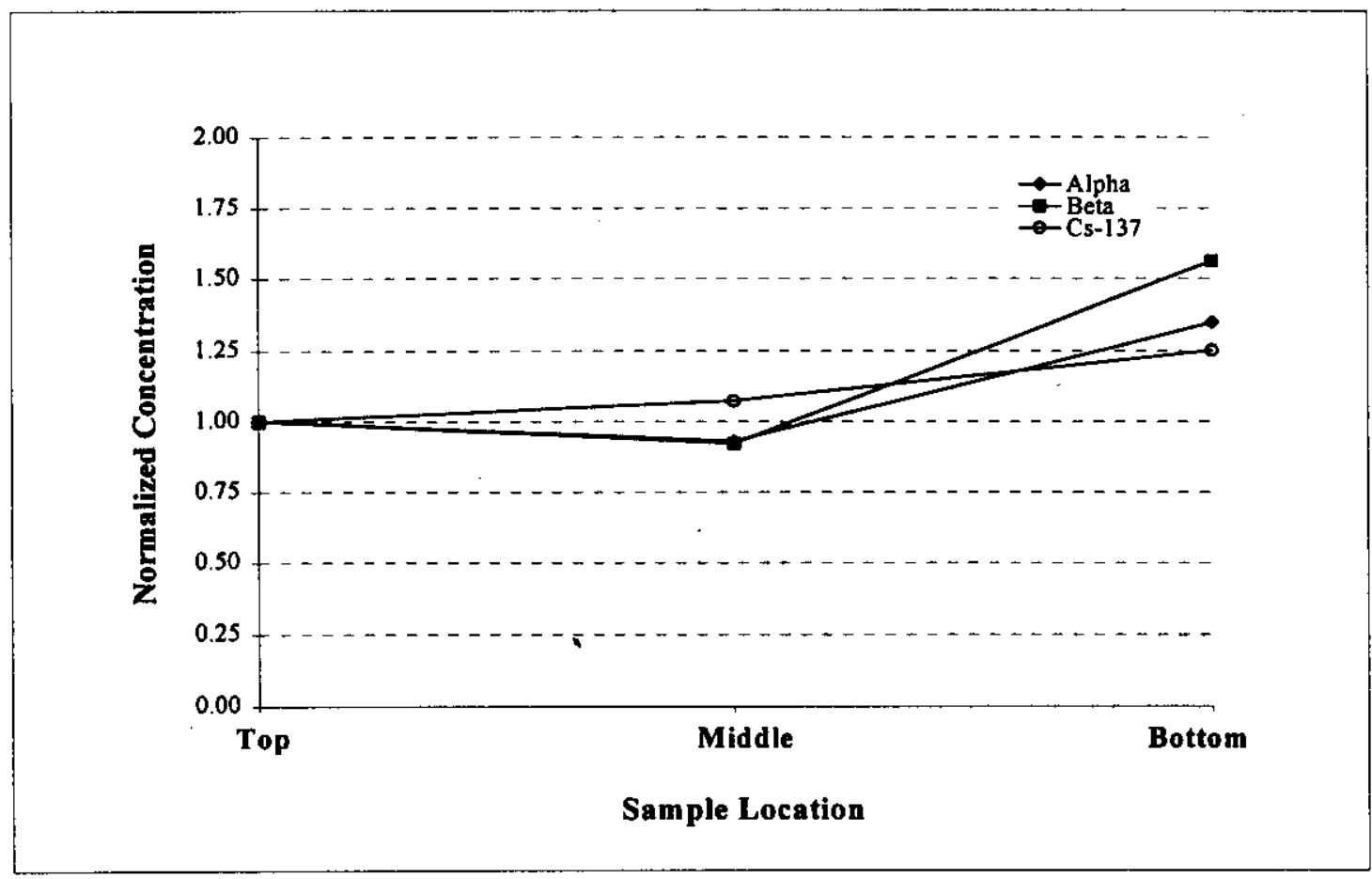

Figure 3-6 Normalized Radiochemical Concentrations as a Function of Settling (Duplicate Sample)

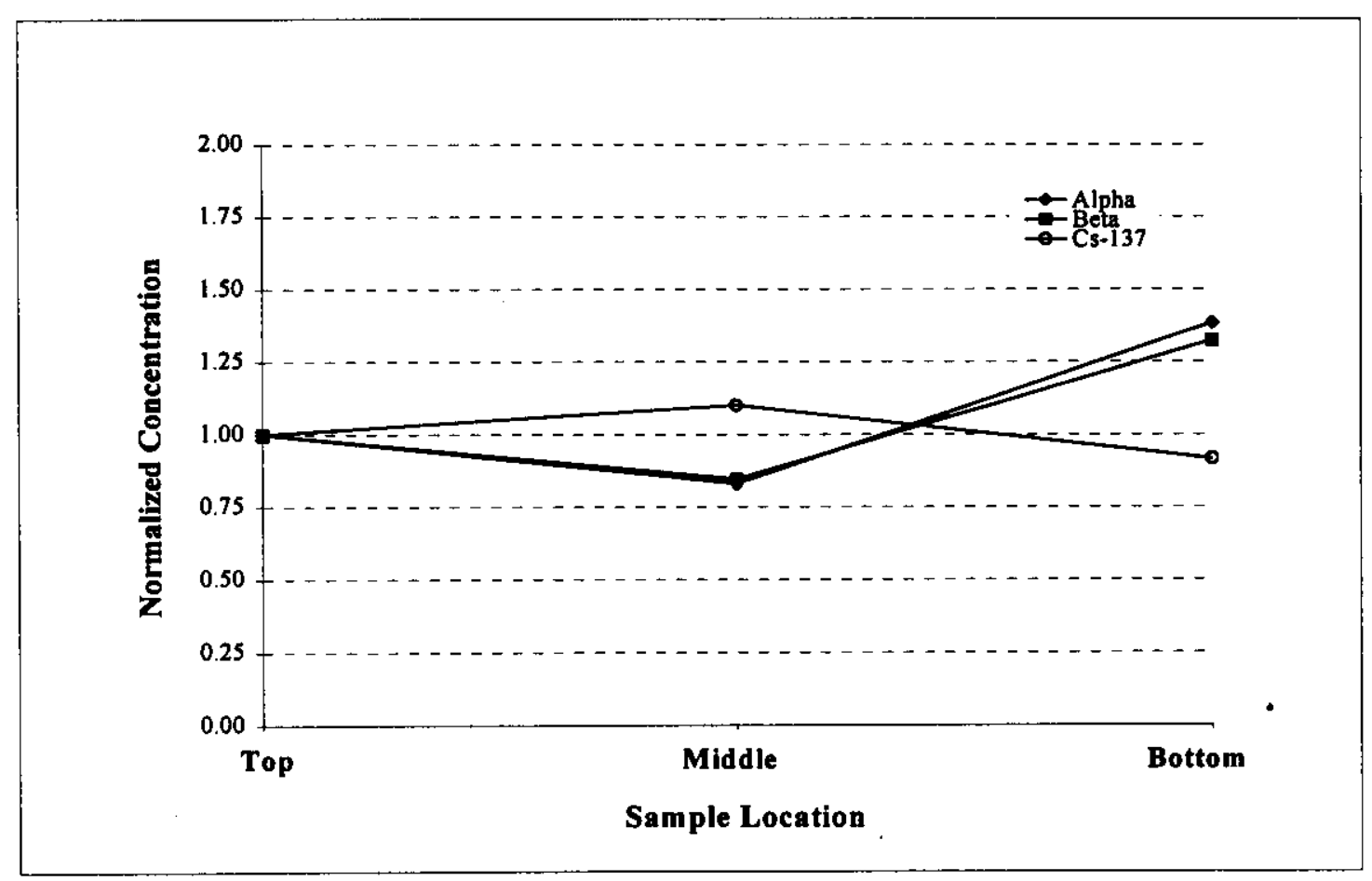


RPP-5798, Rev. 0

\subsection{REFERENCES}

Baldwin, J. H., (1997), Tank Characterization Report for Single-Shell Tank 241-C-104, HNF-SD-WM-ER-679, Rev. O, Lockheed Martin Hanford Corporation, Richland, Washington.

Beck, M. A., (1999), Internal Memo 82800-99-009 to L. L. Lockrem, Numatec Hanford Corporation, "Test Procedure, C-104 Sludge Retrieval And Dilution Test", dated June 14, 1999.

Crawford, T. W., (1999), Internal Memo 82400-99-030 to D. L. Herting, Numatec Hanford Corporation, "Letter of Instruction for 241-C-104 Process Test", dated June 14, 1999.

Herting, D. L., (1999), Results of Dilution Studies with Waste from Tank 241-AW-101, HNF-4964, Rev. 0, Numatec Hanford Corporation, Richland, Washington.

Herting, D. L., M. A. Beck, and J. F. O'Rourke, (1999), Test Plan for Tank 241-C-104 Retrieval Testing, HNF-4094, Rev. 1, Numatec Hanford Corporation, Richland, Washington.

MacLean, G. T., (1999), The Settling and Compaction of Nuclear Waste Slurries, HNF-5177, Rev. 0, Fluor Daniel Northwest, Inc., Richland, Washington.

Meznarich, H. K. (1996), Process Chemistry \& Statistics Quality Assurance Plan, WHC-SD-CP-QAPP-018, Rev. 0, Westinghouse Hanford Company, Richland, Washington.

Nguyen, D. M. (1999a), Data Quality Objectives for TWRS Privatization Phase 1: Confirm Tank T is an Appropriate Feed Source for High-Level Waste Feed Batch X, HNF-1558, Rev. 1, PSDQO-02, Lockheed Martin Hanford Corporation, Richland, Washington.

Nguyen, D. M. (1999b), Data Quality Objectives for TWRS Privatization Phase 1: Confirm Tank T is an Appropriate Feed Source for Low-Activity Waste Feed Batch X, HNF-1796, Rev. 2, PSDQO-01, Lockheed Martin Hanford Corporation, Richland, Washington.

Powell, R. W. (1999), Internal Memo LMHC-9955729 to L. L. Lockrem, Numatec Hanford Corporation, "Suspension of Sludge Washing Activities", dated August 17.

Troyer, G. L., and W. I. Winters, 1999, Survey of Technetium Analytical Production Methods Supporting Hanford Nuclear Materials Processing, HNF-5309, Rev. 0, Numatec Hanford Corporation, Richland, Washington 

RPP-5798, Rev. 0

APPENDIX A

VISCOSITY DATA

A-1 
RPP-5798, Rev. 0

VISCOSITY ANALYSIS OF

WHOLE-TANK-COMPOSITE SAMPLE

AT AMBIENT TEMPERATURE

A-2 


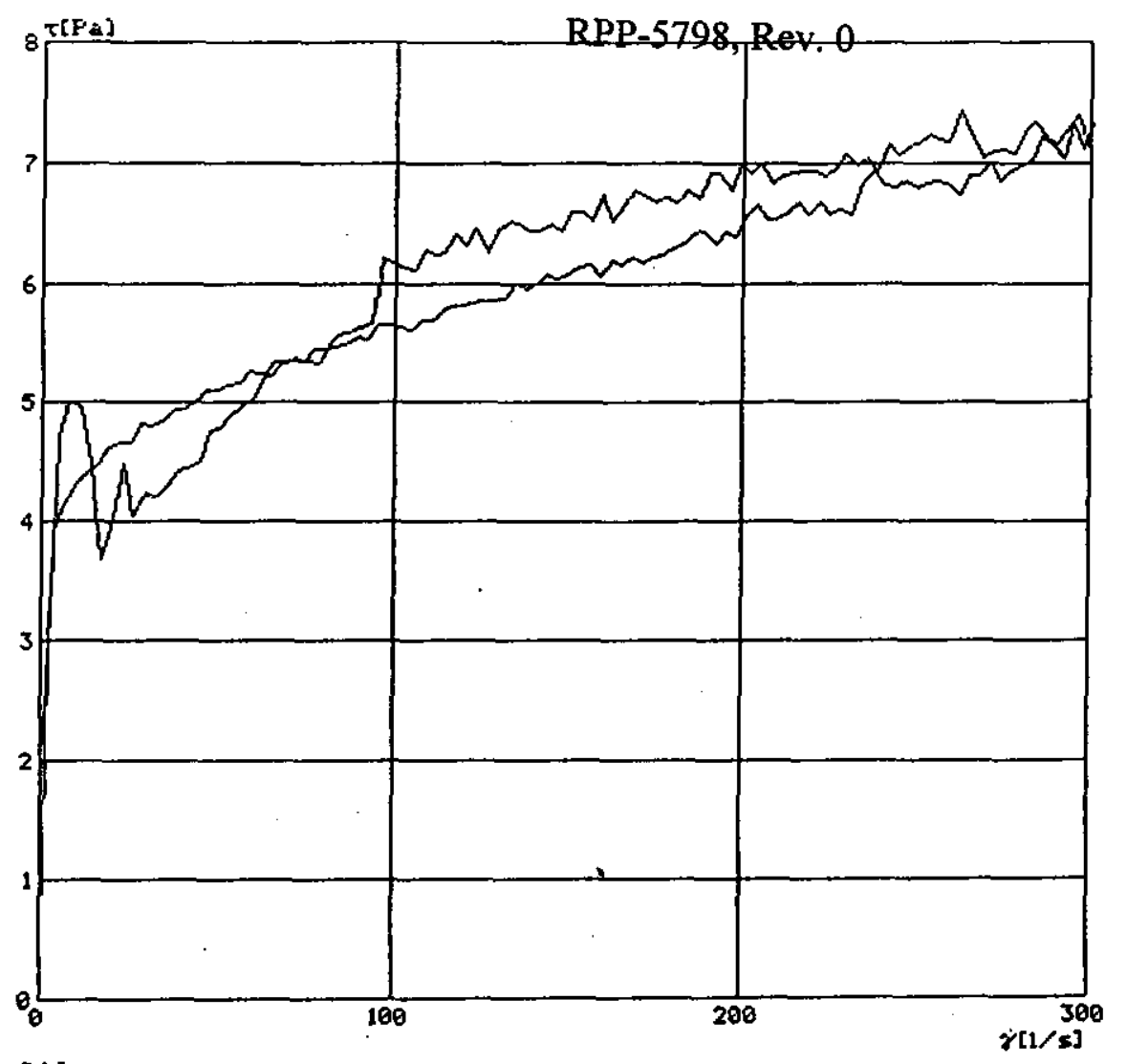

H ค ค $K$ E

Operator:

jwo

Substance:

c104 composite

room temp

Test No.:

01

Test of:

08-10-1999

System:

M5/MV1

Temperature:

$23.0^{\circ} \mathrm{C}$

C104C25.ROT

HAFKE ROE 3.9 .3

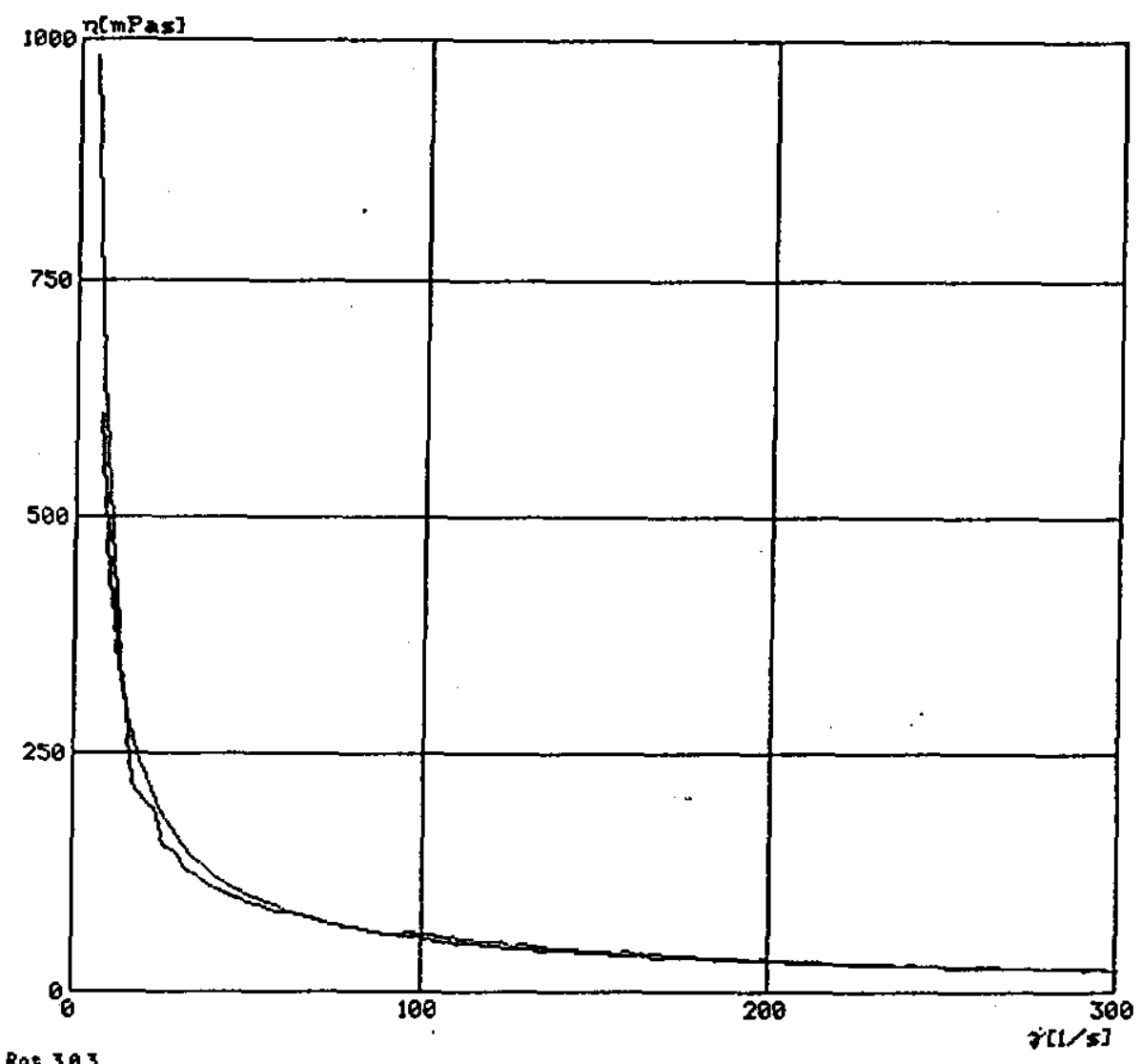

Hคค $X E$

Operator:

Jwo

Substence:

0104 composite

room temp

Test No.:

81

Test of:

88-10-1999

System:

M5/MV1

Temperature:

$23.0^{\circ} \mathrm{C}$

C104C25.ROT 
RPP-5798, Rev. 0

\begin{tabular}{|c|c|c|c|c|}
\hline$\tau$ [Pa] & $\gamma\left[\mathrm{s}^{-3}\right]$ & $\eta[\mathbf{m P a s}]$ & t[min] & $\left.\operatorname{Temp}\right|^{\circ} \mathrm{C}$ \\
\hline 0.000 & 0.464 & 0.00 & 0.00 & 25.1 \\
\hline 1.849 & 2.889 & 1563.00 & 0.03 & 25.1 \\
\hline 4.804 & 4.718 & 982.20 & 0.06 & 25.2 \\
\hline 7.739 & 5.006 & 646.90 & 0.09 & 25.2 \\
\hline 11.000 & 4.973 & 452.20 & 0.12 & 25.2 \\
\hline 14.030 & 4.463 & 318.10 & 0.15 & 25.2 \\
\hline 16.890 & 3.674 & 217.50 & 0.18 & 25.2 \\
\hline 19.960 & 4.009 & 200.90 & 0.21 & 25.2 \\
\hline 23.240 & 4.472 & 192.50 & 0.24 & 25.2 \\
\hline 26.100 & 4.042 & 154.90 & 0.27 & 25.2 \\
\hline 29.150 & 4.241 & 145.50 & 0.30 & 25.2 \\
\hline 32.390 & 4.208 & 129.90 & 0.33 & 25.2 \\
\hline 35.260 & 4.307 & 122.10 & 0.36 & 25.2 \\
\hline 38.350 & 4.411 & 115.00 & 0.39 & 25.2 \\
\hline 41.210 & 4.444 & 107.80 & 0.42 & 25.2 \\
\hline 44.470 & 4.496 & 101.10 & 0.45 & 25.2 \\
\hline 47.580 & 4.756 & 99.96 & 0.48 & 25.2 \\
\hline 50.480 & 4.789 & 94.87 & 0.51 & 25.3 \\
\hline 53.390 & 4.888 & 91.55 & 0.54 & 25.2 \\
\hline 56.800 & 4.959 & 87.30 & 0.57 & 25.2 \\
\hline 59.680 & 5.016 & 84.04 & 0.60 & 25.2 \\
\hline 62.710 & 5.228 & 83.37 & 0.63 & 25.2 \\
\hline 65.970 & 5.342 & 80.97 & 0.67 & 25.2 \\
\hline 68.890 & 5.323 & 77.26 & 0.70 & 25.3 \\
\hline 71.920 & 5.370 & 74.67 & 0.73 & 25.2 \\
\hline 74.820 & 5.356 & 71.58 & 0.75 & 25.2 \\
\hline 78.100 & 5.327 & 68.22 & 0.79 & 25.2 \\
\hline 81.090 & 5.497 & 67.80 & 0.82 & 25.3 \\
\hline 84.040 & 5.568 & 66.26 & 0.85 & 25.2 \\
\hline 86.920 & 5.582 & 64.23 & 0.88 & 25.3 \\
\hline 90.370 & 5.625 & 62.24 & 0.91 & 25.3 \\
\hline 93.270 & 5.677 & 60.87 & 0.94 & 25.3 \\
\hline 96.150 & 6.216 & 64.65 & 0.97 & 25.3 \\
\hline 99.620 & 6.145 & 61.68 & 1.00 & 25.3 \\
\hline 102.500 & 6.131 & 59.84 & 1.03 & 25.3 \\
\hline 105.400 & 6.121 & 58.08 & 1.06 & 25.3 \\
\hline 108.500 & 6.277 & 57.86 & 1.09 & 25.3 \\
\hline 111.800 & 6.235 & 55.79 & 1.12 & 25.3 \\
\hline 113.800 & 6.249 & 54.91 & 1.15 & 25.3 \\
\hline 117.200 & 6.409 & 54.67 & 1.18 & 25.3 \\
\hline 120.300 & 6.305 & 52.42 & 1.21 & 25.3 \\
\hline 123.100 & 6.457 & 52.43 & 1.24 & 25.3 \\
\hline 126.400 & 6.244 & 49.41 & 1.27 & 25.3 \\
\hline 129.200 & 6.433 & 49.78 & 1.30 & 25.3 \\
\hline 132.500 & 6.523 & 49.24 & 1.33 & 25.3 \\
\hline 135.500 & 6.490 & 47.88 & 1.36 & 25.3 \\
\hline 138.400 & 6.438 & 46.52 & 1.39 & 25.3 \\
\hline 141.200 & 6.438 & 45.58 & 1.42 & 25.3 \\
\hline 144.700 & 6.504 & 44.95 & 1.45 & 25.3 \\
\hline 147.500 & 6.433 & 43.60 & 1.48 & 25.3 \\
\hline 150.400 & 6.589 & 43.81 & 1.51 & 25.3 \\
\hline 153.800 & 6.598 & 42.89 & 1.55 & 25.4 \\
\hline 156.700 & 6.518 & 41.60 & 1.58 & 25.4 \\
\hline 159.500 & 6.735 & 42.22 & 1.60 & 25.4 \\
\hline 162.400 & 6.513 & 40.11 & 1.63 & 25.4 \\
\hline 165.800 & 6.664 & 40.19 & 1.67 & 25.3 \\
\hline 168.700 & 6.787 & 40.23 & 1.70 & 25.3 \\
\hline 171.600 & 6.731 & 39.23 & 1.73 & 25.3 \\
\hline 174.400 & 6.679 & 38.29 & 1.75 & 25.4 \\
\hline 177.800 & 6.712 & 37.74 & 1.79 & 25.4 \\
\hline 180.700 & 6.669 & 36.91 & 1.82 & 25.4 \\
\hline 183.600 & 6.768 & 36.87 & 1.85 & 25.4 \\
\hline 187.000 & 6.698 & 35.82 & 1.88 & 25.3 \\
\hline 189.900 & 6.910 & 36.40 & 1.91 & 25.3 \\
\hline 192.700 & 6.910 & 35.86 & 1.94 & 25.3 \\
\hline 196.100 & 6.787 & 34.60 & 1.97 & 25.3 \\
\hline
\end{tabular}


RPP-5798, Rev. 0

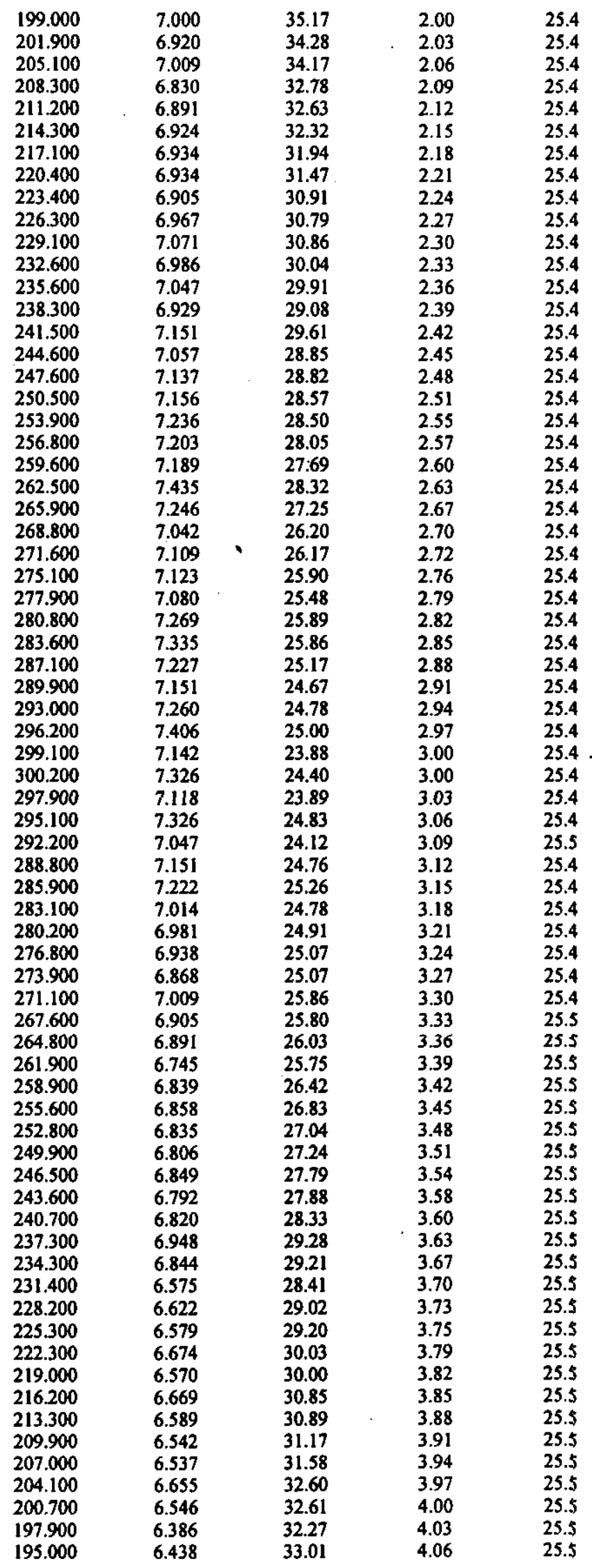


RPP-5798, Rev. 0

\begin{tabular}{|c|c|c|c|c|}
\hline 192.100 & 6.338 & 32.99 & 4.09 & 25.5 \\
\hline 188.700 & 6.442 & 34.14 & 4.12 & 25.5 \\
\hline 185.900 & 6.419 & 34.54 & 4.15 & 25.5 \\
\hline 183.000 & 6.343 & 34.66 & 4.18 & 25.6 \\
\hline 179.600 & 6.296 & 35.06 & 4.21 & 25.5 \\
\hline 176.700 & 6.239 & 35.31 & 4.24 & 25.5 \\
\hline 173.800 & 6.206 & 35.70 & 4.27 & 25.5 \\
\hline 171.000 & 6.168 & 36.08 & 4.30 & 25.6 \\
\hline 167.600 & 6.220 & 37.12 & 4.33 & 25.6 \\
\hline 164.700 & 6.149 & 37.34 & 4.36 & 25.6 \\
\hline 161.800 & 6.192 & 38.26 & 4.39 & 25.6 \\
\hline 159.000 & 6.055 & 38.09 & 4.42 & 25.6 \\
\hline 155.500 & 6.145 & 39.51 & 4.45 & 25.6 \\
\hline 152.500 & 6.131 & 40.20 & 4.48 & 25.6 \\
\hline 149.400 & 6.064 & 40.58 & 4.51 & 25.6 \\
\hline 146.200 & 6.036 & 41.29 & 4.55 & 25.6 \\
\hline 143.500 & 6.074 & 42.32 & 4.58 & 25.6 \\
\hline 140.500 & 5.998 & 42.70 & 4.60 & 25.6 \\
\hline 137.400 & 5.942 & 43.23 & 4.63 & 25.6 \\
\hline 134.200 & 5.989 & 44.63 & 4.67 & 25.6 \\
\hline 131.100 & 5.861 & 44.69 & 4.70 & 25.6 \\
\hline 128.300 & 5.852 & 45.62 & 4.73 & 25.6 \\
\hline 125.200 & 5.842 & 46.65 & 4.75 & 25.6 \\
\hline 122.200 & 5.838 & 47.78 & 4.79 & 25.6 \\
\hline 118.900 & 5.814 & 48.88 & 4.82 & 25.6 \\
\hline 116.100 & 5.819 & 50.12 & 4.85 & 25.6 \\
\hline 112.800 & 5.743 & 50.89 & 4.88 & 25.6 \\
\hline 111.000 & 5.682 & 51.18 & 4.91 & 25.6 \\
\hline 107.700 & 5.691 & 52.86 & 4.94 & 25.6 \\
\hline 104.600 & 5.616 & 53.66 & 4.97 & 25.6 \\
\hline 101.400 & 5.634 & 55.57 & 5.00 & 25.6 \\
\hline 98.450 & 5.649 & 57.37 & 5.03 & 25. \\
\hline 95.350 & 5.653 & 59.29 & 5.06 & 25. \\
\hline 92.110 & 5.535 & 60.10 & 5.09 & 25.6 \\
\hline 89.190 & 5.545 & 62.17 & 5.12 & 25.6 \\
\hline 86.120 & 5.479 & 63.62 & 5.15 & 25.6 \\
\hline 83.220 & 5.474 & 65.77 & 5.18 & 25.6 \\
\hline 79.750 & 5.450 & 68.34 & 5.21 & 25. \\
\hline 76.910 & 5.455 & 70.92 & 5.24 & 25.6 \\
\hline 74.050 & 5.351 & 72.26 & 5.27 & 25.6 \\
\hline 71.000 & 5.337 & 75.16 & 5.30 & 25.6 \\
\hline 67.710 & 5.351 & 79.03 & 5.33 & 25.6 \\
\hline 64.790 & 5.228 & 80.69 & 5.36 & 25.6 \\
\hline 61.760 & 5.242 & 84.88 & 5.39 & 25.6 \\
\hline 58.440 & 5.271 & 90.19 & 5.42 & 25. \\
\hline 55.580 & 5.162 & 92.87 & 5.45 & 25. \\
\hline 52.480 & 5.138 & 97.92 & 5.48 & 25.6 \\
\hline 49.600 & 5.101 & 102.80 & 5.51 & 25.6 \\
\hline 46.340 & 5.096 & 110.00 & 5.55 & 25.6 \\
\hline 43.250 & 5.006 & 115.70 & 5.57 & 25.6 \\
\hline 40.370 & 4.949 & 122.60 & 5.60 & 25. \\
\hline 37.380 & 4.935 & 132.00 & 5.63 & 25. \\
\hline 34.060 & 4.836 & 142.00 & 5.67 & 25. \\
\hline 31.180 & 4.808 & 154.20 & 5.70 & 25.6 \\
\hline 28.170 & 4.827 & 171.30 & 5.72 & 25.6 \\
\hline 24.880 & 4.671 & 187.80 & 5.76 & 25.6 \\
\hline 22.000 & 4.671 & 212.30 & 5.79 & 25.6 \\
\hline 19.020 & 4.614 & 242.50 & 5.82 & 25. \\
\hline 16.130 & 4.477 & 277.60 & 5.85 & 25. \\
\hline 12.830 & 4.401 & 343.10 & 5.88 & 25 \\
\hline 9.855 & 4.312 & 437.50 & 5.91 & 25.6 \\
\hline 6.900 & 4.184 & 606.40 & 5.94 & 25.7 \\
\hline 3.641 & 3.919 & 1077.00 & 5.97 & 25.6 \\
\hline 0.324 & 2.169 & 6695.00 & 6.00 & 25.6 \\
\hline
\end{tabular}


RPP-5798, Rev. 0

\section{VISCOSITY ANALYSIS OF}

WHOLE-TANK-COMPOSITE SAMPLE AT $45^{\circ} \mathrm{C}$ 


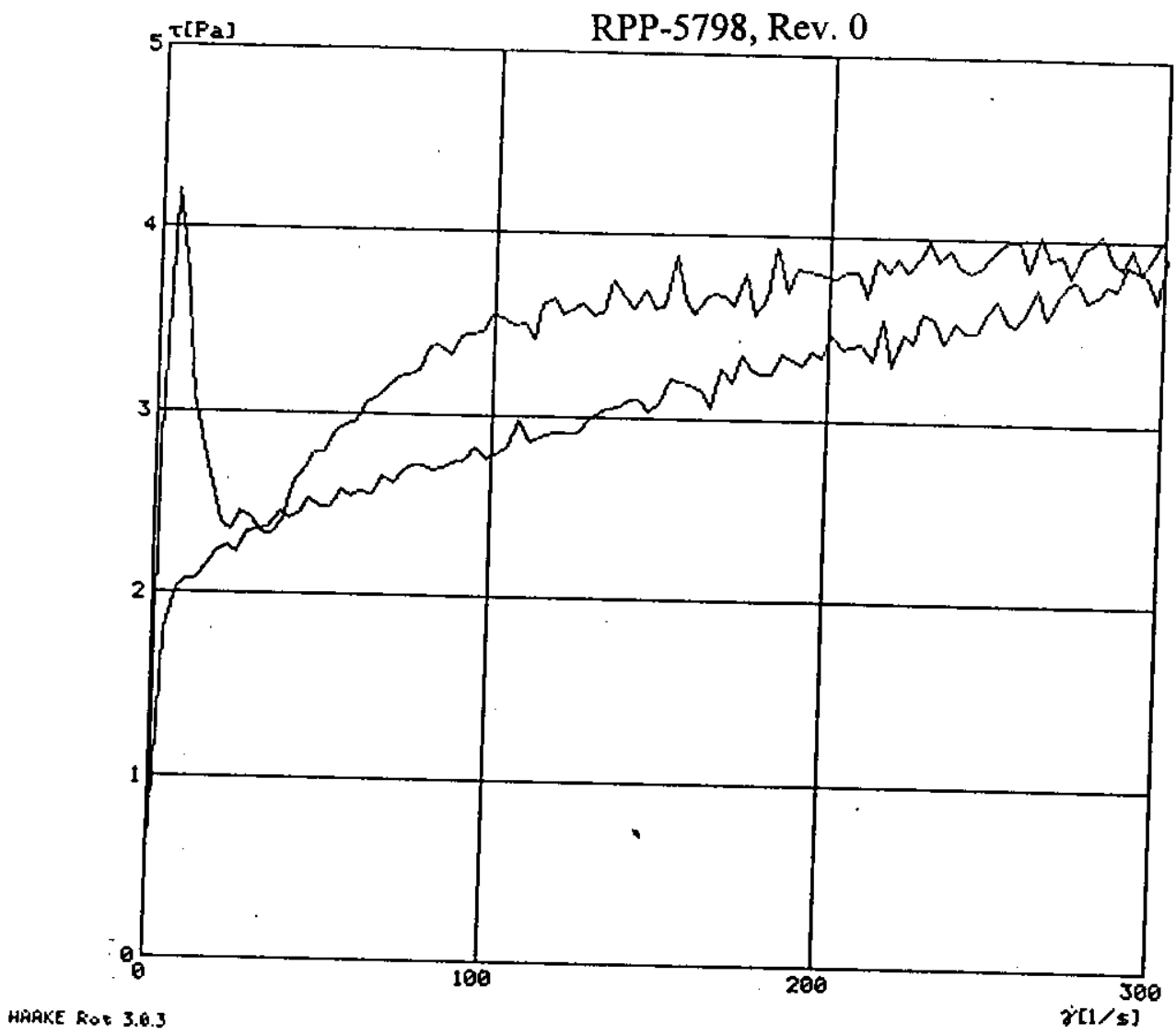

H A $R$ K E

Oxerator:

jwe

Substance:

c104 composite

$45 \mathrm{C}$

Test Ho.:

a1

Test of:

08-10-1999

System:

M5/MV1

Temperature:

$23.0^{\circ} \mathrm{C}$

D- C104C45.ROT

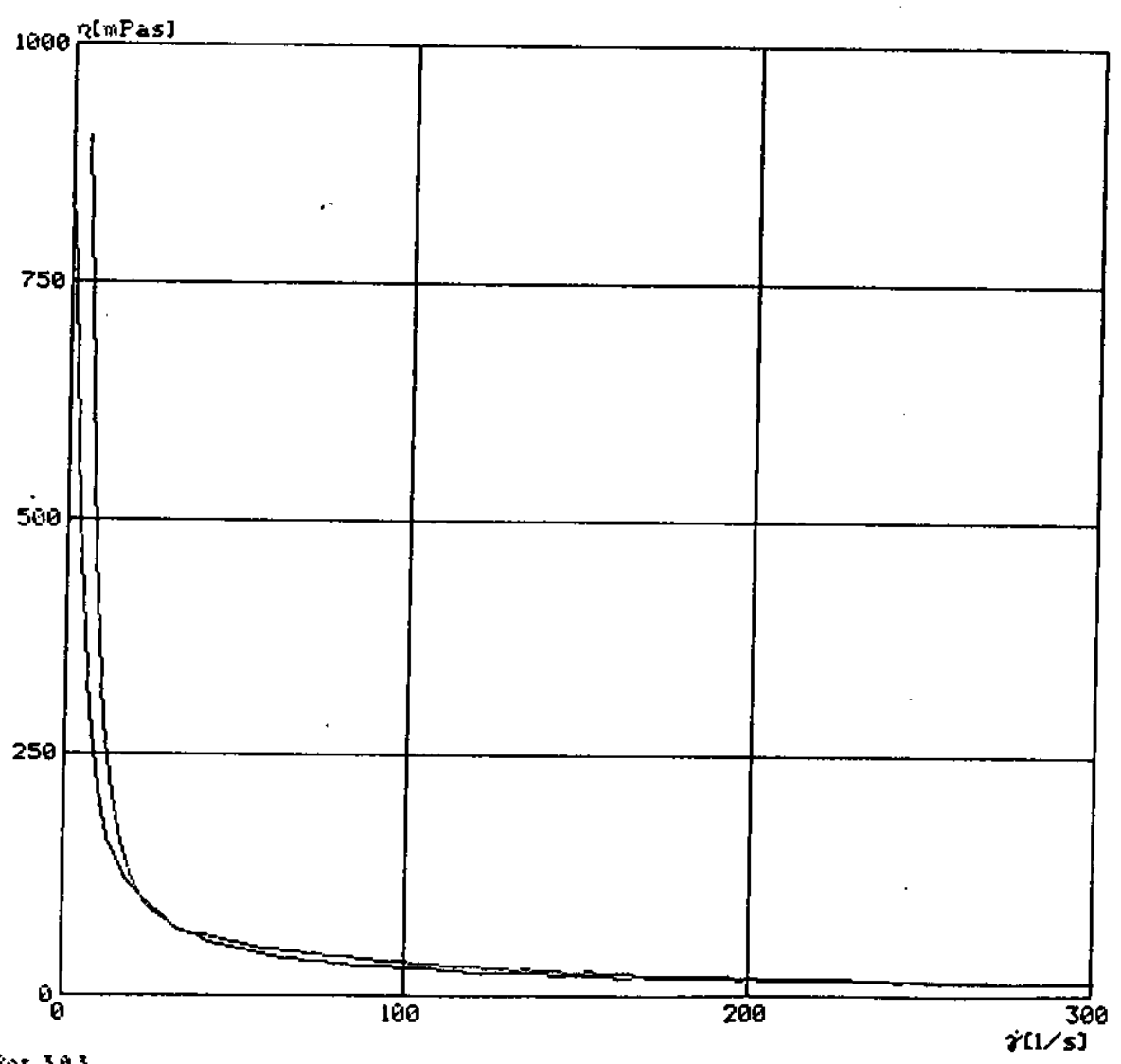

H A $\boldsymbol{A} \times \mathbf{E}$

Operator:

Jwe

Substence:

cl04 composite

45C

Test No:

01

Test of:

08-10-1999

System:

M5/MV1

Temperature:

$23.0^{\circ} \mathrm{C}$

- C104C45.ROT 
RPP-5798, Rev. 0

\begin{tabular}{|c|c|c|c|c|}
\hline$\tau[\mathbf{P a}]$ & $\gamma\left[s^{-1}\right]$ & $\eta[m P a s]$ & $t[m i n]$ & $\left.\operatorname{Temp} /{ }^{\circ} \mathrm{C}\right]$ \\
\hline 0.000 & 0.000 & 0.00 & 0.00 & 45.0 \\
\hline 1.544 & 2.792 & 1808.00 & 0.03 & 44.9 \\
\hline 4.632 & 4.195 & 905.60 & 0.06 & 44.9 \\
\hline 8.044 & 3.746 & 465.70 & 0.09 & 45.0 \\
\hline 10.920 & 3.094 & 283.30 & 0.12 & 45.0 \\
\hline 13.840 & 2.863 & 206.80 & 0.15 & 45.0 \\
\hline 16.770 & 2.651 & 158.00 & 0.18 & 45.0 \\
\hline 20.030 & 2.397 & 119.60 & 0.21 & 45.0 \\
\hline 23.030 & 2,353 & 102.20 & 0.24 & 44.9 \\
\hline 26.040 & 2.465 & 94.66 & 0.27 & 45.0 \\
\hline 29.280 & 2.427 & 82.91 & 0.30 & 45.0 \\
\hline 32.200 & 2.343 & 72.78 & 0.33 & 45.0 \\
\hline 35.240 & 2.330 & 66.10 & 0.36 & 45.0 \\
\hline 38.100 & 2.411 & 63.27 & 0.39 & 45.0 \\
\hline 41.380 & 2.614 & 63.15 & 0.42 & 45.0 \\
\hline 44.490 & 2.699 & 60.67 & 0.45 & 45.0 \\
\hline 47.250 & 2.787 & 58.98 & 0.48 & 45.0 \\
\hline 50.130 & 2.782 & 55.50 & 0.51 & 45.0 \\
\hline 53.680 & 2.896 & 53.94 & 0.54 & 45.0 \\
\hline 56.560 & 2.933 & 51.87 & 0.57 & 45.0 \\
\hline 59.510 & 2.952 & 49.61 & 0.60 & 45.0 \\
\hline 62.900 & 3.066 & 48.74 & 0.64 & 45.0 \\
\hline 65.760 . & 3.085 & 46.90 & 0.67 & 45.0 \\
\hline $68.890^{\circ}$ & 3.151 & 45.74 & 0.69 & 45.0 \\
\hline 71.790 & 3.198 & 44.55 & 0.72 & 45.0 \\
\hline 75.030 & 3.212 & 42.81 & 0.76 & 45.0 \\
\hline 78.080 & 3.236 & 41.44 & 0.79 & 45.0 \\
\hline 80.990 & 3.368 & 41.58 & 0.82 & 45.0 \\
\hline 83.910 & 3.382 & 40.31 & 0.84 & 44.9 \\
\hline 87.320 & 3.326 & 38.08 & 0.88 & 44.9 \\
\hline 90.240 & 3.425 & 37.95 & 0.91 & 44.9 \\
\hline 93.120 & 3.444 & 36.98 & 0.94 & 44.9 \\
\hline 96.590 & 3.453 & 35.75 & 0.97 & 45.0 \\
\hline 99.440 & 3.538 & 35.58 & 1.00 & 45.0 \\
\hline 102.400 & 3.533 & 34.51 & 1.03 & 44.9 \\
\hline 105.400 & 3.491 & 33.13 & 1.06 & 44.9 \\
\hline 108.700 & 3.510 & 32.29 & 1.09 & 45.0 \\
\hline 111.700 & 3.415 & 30.58 & 1.12 & 45.0 \\
\hline 113.800 & 3.609 & 31.71 & 1.15 & 45.0 \\
\hline 117.200 & 3.642 & 31.07 & 1.18 & 45.0 \\
\hline 120.300 & 3.557 & 29.57 & 1.21 & 45.0 \\
\hline 123.300 & 3.595 & 29.15 & 1.24 & 45.0 \\
\hline 125.800 & 3.618 & 28.76 & 1.27 & 45.0 \\
\hline 129.200 & 3.562 & 27.56 & 1.30 & 45.0 \\
\hline 132.300 & 3.571 & 27.00 & 1.33 & 45.0 \\
\hline 135.100 & 3.741 & 27.68 & 1.36 & 45.0 \\
\hline 138.400 & 3.661 & 26.45 & 1.39 & 45.0 \\
\hline 141.400 & 3.590 & 25.38 & 1.42 & 45.0 \\
\hline 144.500 & 3.694 & 25.57 & 1.45 & 45.0 \\
\hline 147.500 & 3.600 & 24.40 & 1.48 & 45.0 \\
\hline 150.600 & 3.609 & 23.97 & 1.52 & 45.0 \\
\hline 153.600 & 3.878 & 25.24 & 1.54 & 45.0 \\
\hline 156.700 & 3.647 & 23.27 & 1.57 & 45.0 \\
\hline 159.500 & 3.571 & 22.38 & 1.60 & 45.0 \\
\hline 162.800 & 3.661 & 22.49 & 1.64 & 45.0 \\
\hline 165.800 & 3.685 & 22.22 & 1.67 & 45.0 \\
\hline 168.700 & 3.675 & 21.79 & 1.69 & 45.0 \\
\hline 171.600 & 3.623 & 21.12 & 1.72 & 45.0 \\
\hline 175.000 & 3.798 & 21.70 & 1.76 & 45.0 \\
\hline 177.800 & 3.566 & 20.05 & 1.79 & 45.0 \\
\hline 180.700 & 3.656 & 20.23 & 1.82 & 45.0 \\
\hline 184.100 & 3.930 & 21.34 & 1.85 & 45.0 \\
\hline 187.000 & 3.694 & 19.75 & 1.88 & 45.0 \\
\hline 189.900 & 3.826 & 20.15 & 1.91 & 45.0 \\
\hline 192.700 & 3.807 & 19.76 & 1.94 & 45.0 \\
\hline 196.100 & 3.793 & 19.34 & 1.97 & 45.0 \\
\hline
\end{tabular}


RPP-5798, Rev, 0

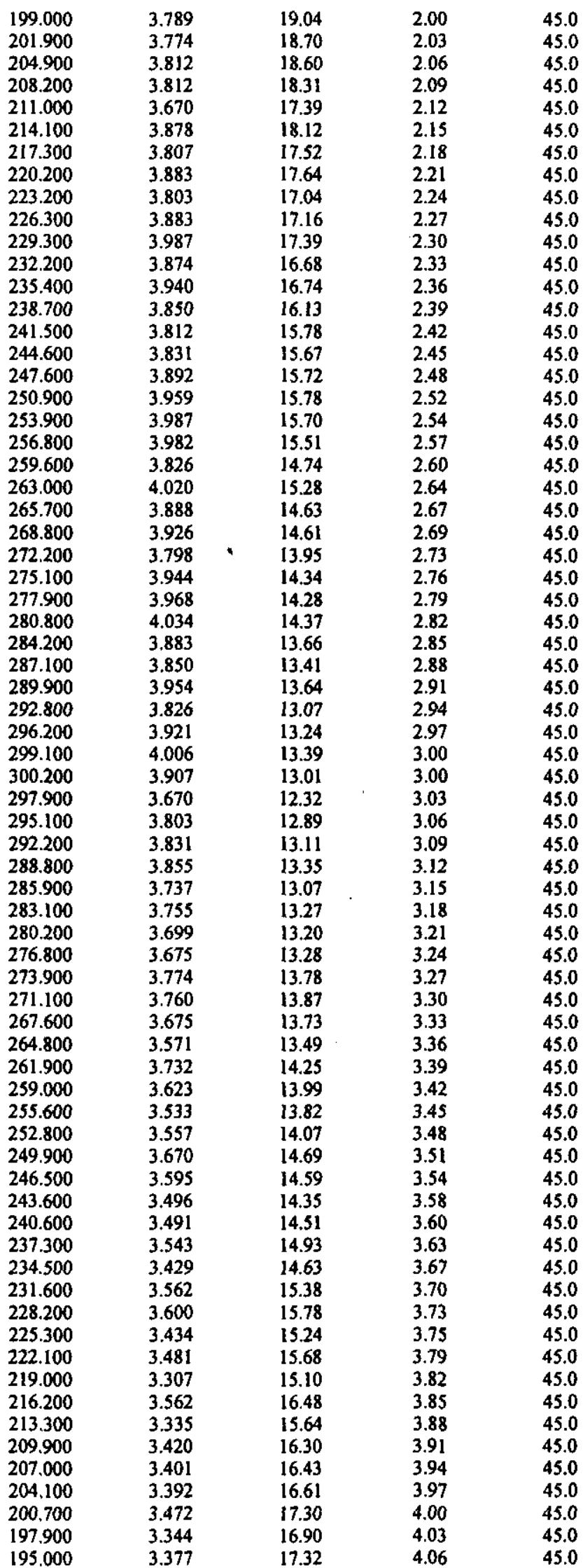


RPP-5798, Rev. 0

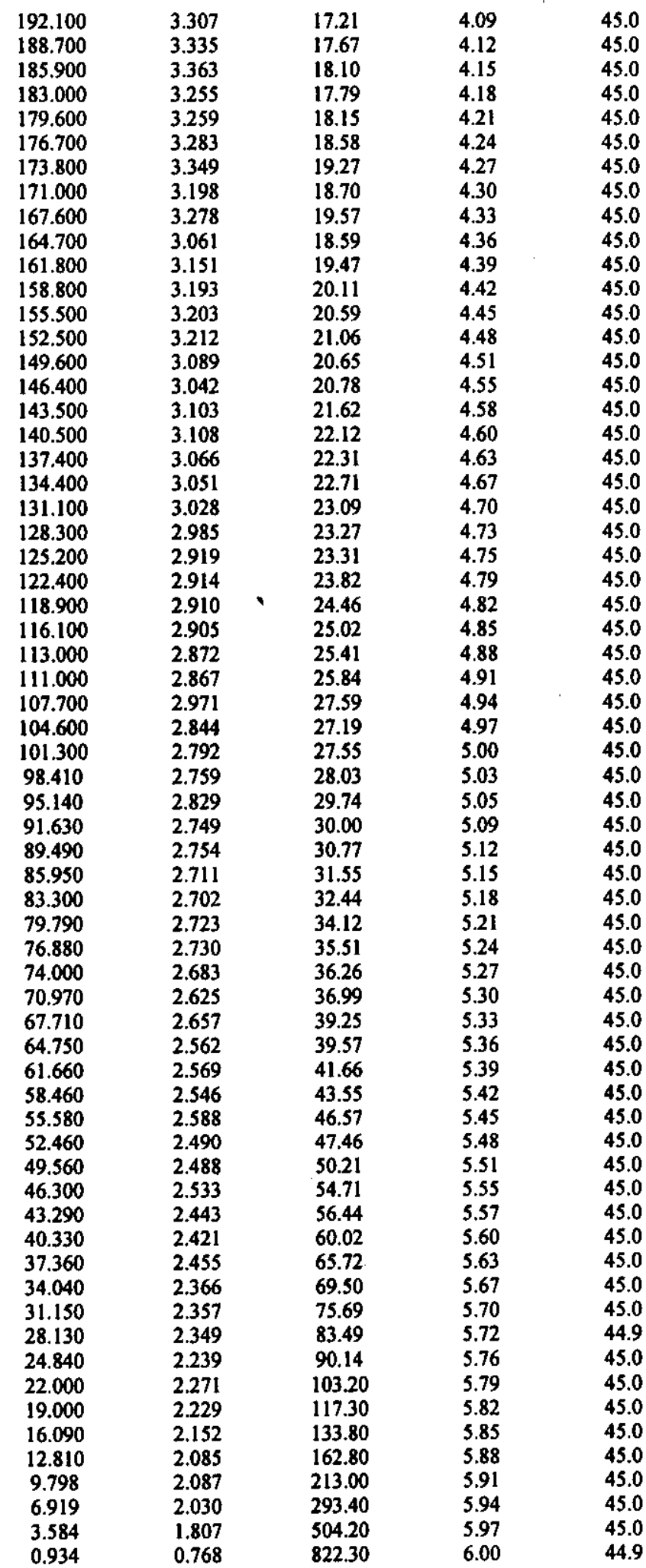


RPP-5798, Rev. 0

\section{VISCOṠITY ANALYSIS OF}

WHOLE-TANK-COMPOSITE SAMPLE AT $65^{\circ} \mathrm{C}$ 
RPP-5798, Rev. 0

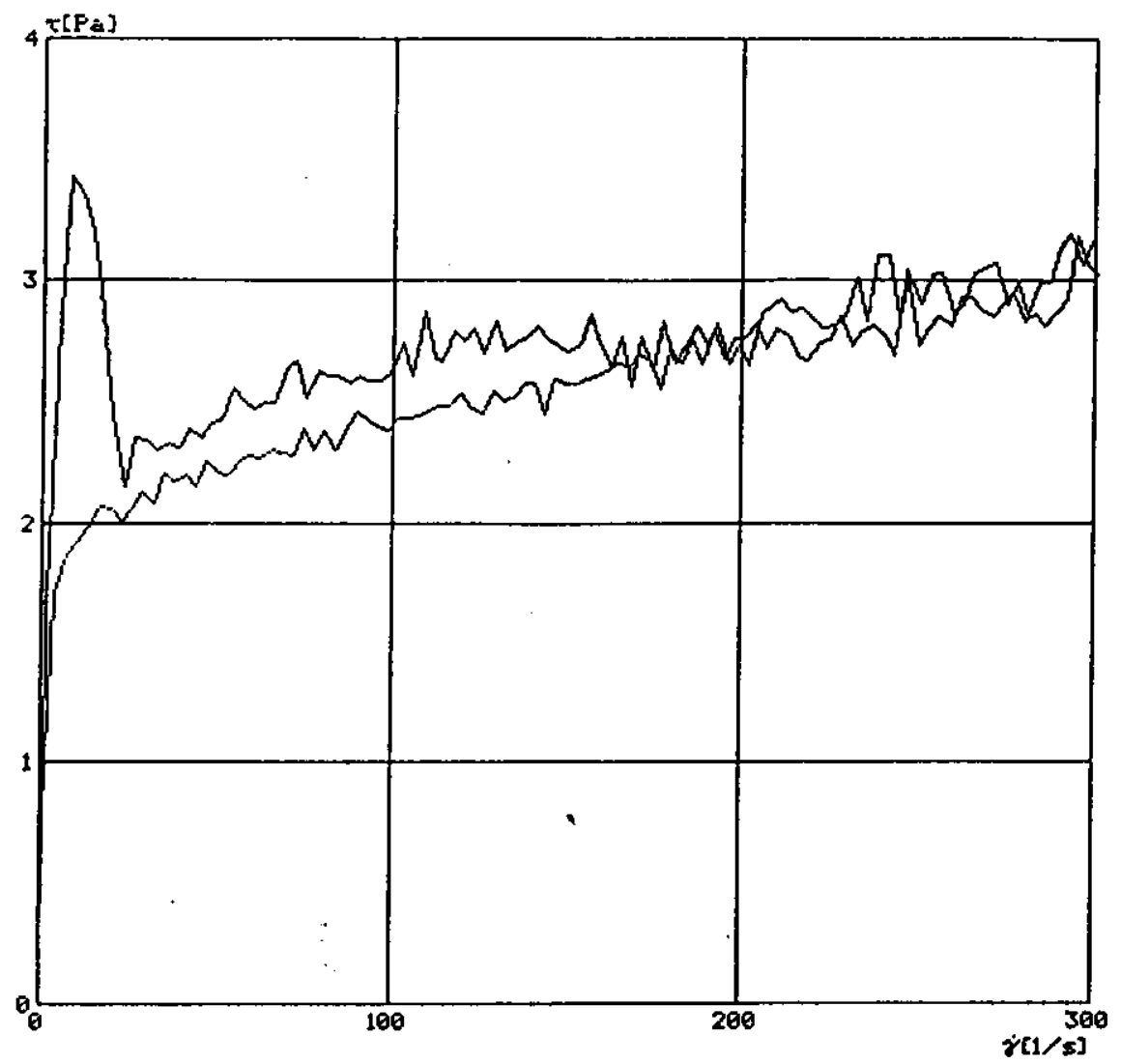

H A $A$ K

Operator:

Jwo

Substance:

cl64 composite

65C

Test No.:

o1

Test of:

08-10-1999

System:

MS/MV1

Temperature:

$23.0^{\circ} \mathrm{C}$

C104C65.ROT

HaQXF Dent 393

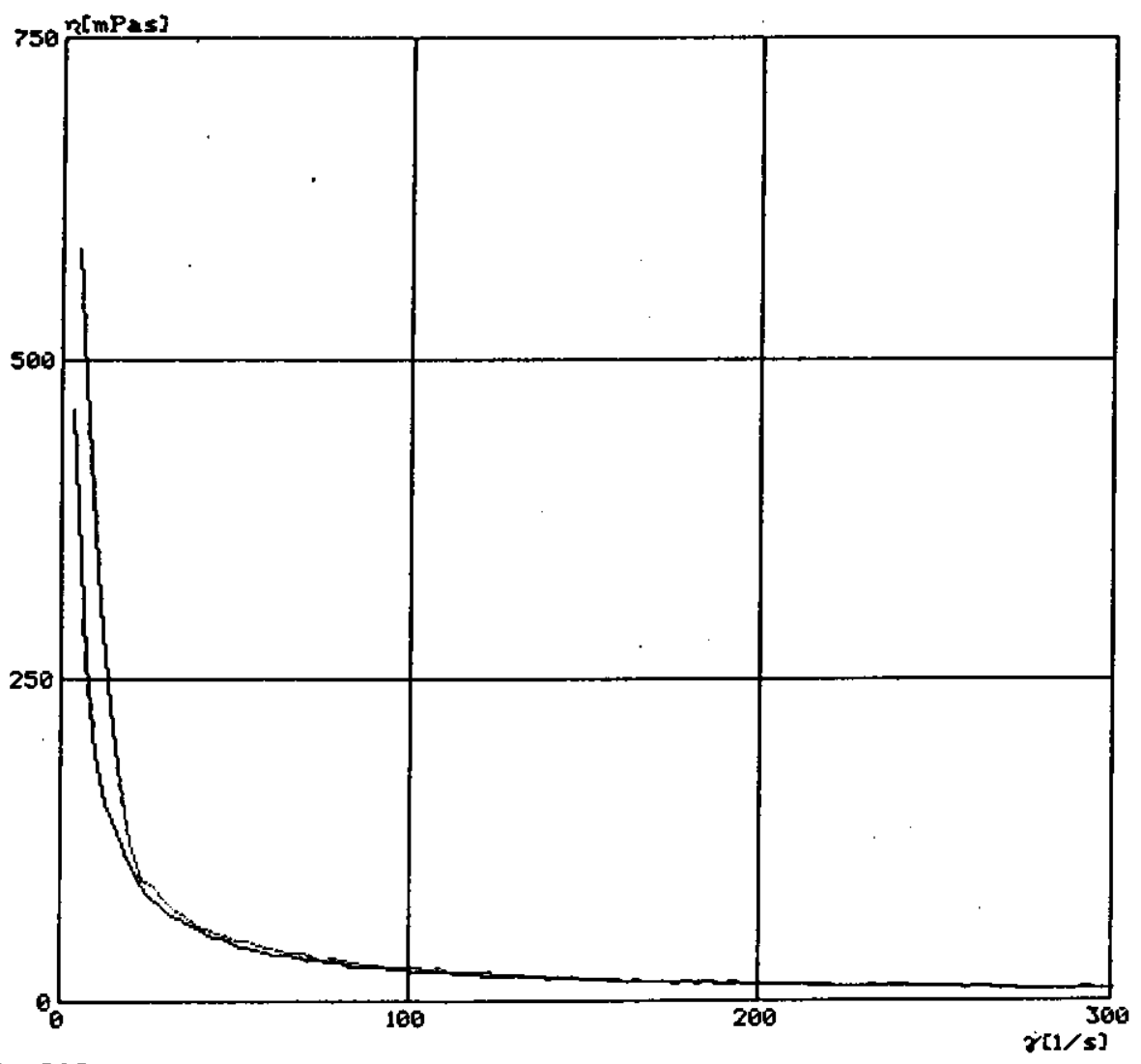

H A $A \mathrm{~K}$

Oxerator:

jwe

Substance:

c104 composite

$65 \mathrm{C}$

Test No.:

o1

Test of:

08-10-1999

System:

M5/MV1

Temperature:

$23.0^{\circ} \mathrm{C}$ 
RPP-5798, Rev. 0

\begin{tabular}{|c|c|c|c|c|}
\hline$\tau$ [Pa] & $\gamma\left[\mathbf{s}^{-1}\right]$ & $\eta[\mathrm{mPas}]$ & t[min] & $\left.\left.\operatorname{Temp}\right|^{\circ} \mathrm{C}\right]$ \\
\hline 0.000 & 0.000 & 0.00 & 0.00 & 65.0 \\
\hline 1.582 & 1.682 & 1063.00 & 0.03 & 65.1 \\
\hline 4.842 & 2.842 & 586.90 & 0.06 & 65.2 \\
\hline 7.567 & 3.428 & 453.00 & 0.09 & 65.3 \\
\hline 10.880 & 3.353 & 308.10 & 0.12 & 65.3 \\
\hline 14.010 & 3.187 & 227.50 & 0.15 & 65.4 \\
\hline 16.890 & 2.863 & 169.60 & 0.18 & 65.5 \\
\hline 19.820 & 2.437 & 122.90 & 0.21 & 65.6 \\
\hline 22.870 & 2.152 & 94.10 & 0.23 & 65.6 \\
\hline 26.060 & 2.356 & 90.40 & 0.27 & 65.7 \\
\hline 29.320 & 2.336 & 79.67 & 0.30 & 65.8 \\
\hline 32.250 & 2.301 & 71.35 & 0.33 & 65.9 \\
\hline 35.280 & 2.331 & 66.05 & 0.36 & 65.9 \\
\hline 38.220 & 2.308 & 60.40 & 0.39 & 66.0 \\
\hline 41.480 & 2.393 & 57.69 & 0.42 & 66.1 \\
\hline 44.490 & 2.356 & 52.95 & 0.45 & 66.2 \\
\hline 47.410 & 2.407 & 50.78 & 0.48 & 66.3 \\
\hline 50.380 & 2.419 & 48.01 & 0.51 & 66.3 \\
\hline 53.720 & 2.550 & 47.48 & 0.54 & 66.4 \\
\hline 56.630 & 2.497 & 44.10 & 0.57 & 66.5 \\
\hline 59.590 & 2.476 & 41.55 & 0.60 & 66.5 \\
\hline 62.940 & 2.494 & 39.63 & 0.64 & 66.6 \\
\hline 65.840 & 2.495 & 37.90 & 0.67 & 66.7 \\
\hline 68.910 & 2.628 & 38.14 & 0.69 & 66.7 \\
\hline 71.800 & 2.665 & 37.11 & 0.72 & 66.7 \\
\hline 75.050 & 2.508 & 33.42 & 0.76 & 66.7 \\
\hline 78.110 & 2.627 & 33.63 & 0.79 & 66.7 \\
\hline 81.010 & 2.601 & 32.10 & 0.82 & 66.7 \\
\hline 83.990 & 2.604 & 31.01 & 0.84 & 66.8 \\
\hline 87.360 & 2.570 & 29.42 & 0.88 & 66.8 \\
\hline 90.240 & 2.602 & 28.83 & 0.91 & 66.8 \\
\hline 93.170 & 2.578 & 27.67 & 0.94 & 66.8 \\
\hline 96.590 & 2.580 & 26.71 & 0.97 & 66.8 \\
\hline 99.460 & 2.614 & 26.29 & 1.00 & 66.8 \\
\hline 102.400 & 2.731 & 26.67 & 1.03 & 66.8 \\
\hline 105.400 & 2.602 & 24.68 & 1.06 & 66.8 \\
\hline 108.700 & 2.861 & 26.32 & 1.09 & 66.8 \\
\hline 111.800 & 2.677 & 23.95 & 1.12 & 66.8 \\
\hline 114.000 & 2.660 & 23.33 & 1.15 & 66.8 \\
\hline 117.200 & 2.787 & 23.78 & 1.18 & 66.8 \\
\hline 120.300 & 2.740 & 22.78 & 1.21 & 66.8 \\
\hline 123.100 & 2.795 & 22.70 & 1.24 & 66.8 \\
\hline 126.000 & 2.689 & 21.34 & 1.27 & 66.8 \\
\hline 129.400 & 2.825 & 21.82 & 1.30 & 66.8 \\
\hline 132.300 & 2.699 & 20.40 & 1,33 & 66.8 \\
\hline 135.100 & 2.731 & 20.21 & 1.36 & 66.8 \\
\hline 138.400 & 2.759 & 19.94 & 1.39 & 66.8 \\
\hline 141.200 & 2.801 & 19.83 & 1.42 & 66.8 \\
\hline 144.300 & 2.752 & 19.07 & 1.45 & 66.9 \\
\hline 147.500 & 2.728 & 18.49 & 1.48 & 66.8 \\
\hline 150.600 & 2.707 & 17.98 & 1.52 & 66.8 \\
\hline 153.600 & 2.733 & 17.79 & 1.54 & 66.8 \\
\hline 156.700 & 2.859 & 18.25 & 1.57 & 66.8 \\
\hline 159.500 & 2.739 & 17.17 & 1.60 & 66.9 \\
\hline 163.000 & 2.640 & 16.20 & 1.64 & 66.8 \\
\hline 165.800 & 2.760 & 16.65 & 1.67 & 66.8 \\
\hline 168.700 & 2.567 & 15.22 & 1.69 & 66.8 \\
\hline 171.600 & 2.760 & 16.09 & 1.72 & 66.8 \\
\hline 175.000 & 2.621 & 14.98 & 1.76 & 66.8 \\
\hline 177.800 & 2.821 & 15.86 & 1.79 & 66.8 \\
\hline 180.700 & 2.657 & 14.70 & 1.82 & 66.8 \\
\hline 184.100 & 2.723 & 14.79 & 1.85 & 66.9 \\
\hline 187.000 & 2.803 & 14.99 & 1.87 & 66.8 \\
\hline 189.900 & 2.726 & 14.36 & 1.91 & 66.9 \\
\hline 192.700 & 2.818 & 14.62 & 1.94 & 66.9 \\
\hline 196.100 & 2.654 & 13.53 & 1.97 & 66.8 \\
\hline
\end{tabular}


RPP-5798, Rev. 0

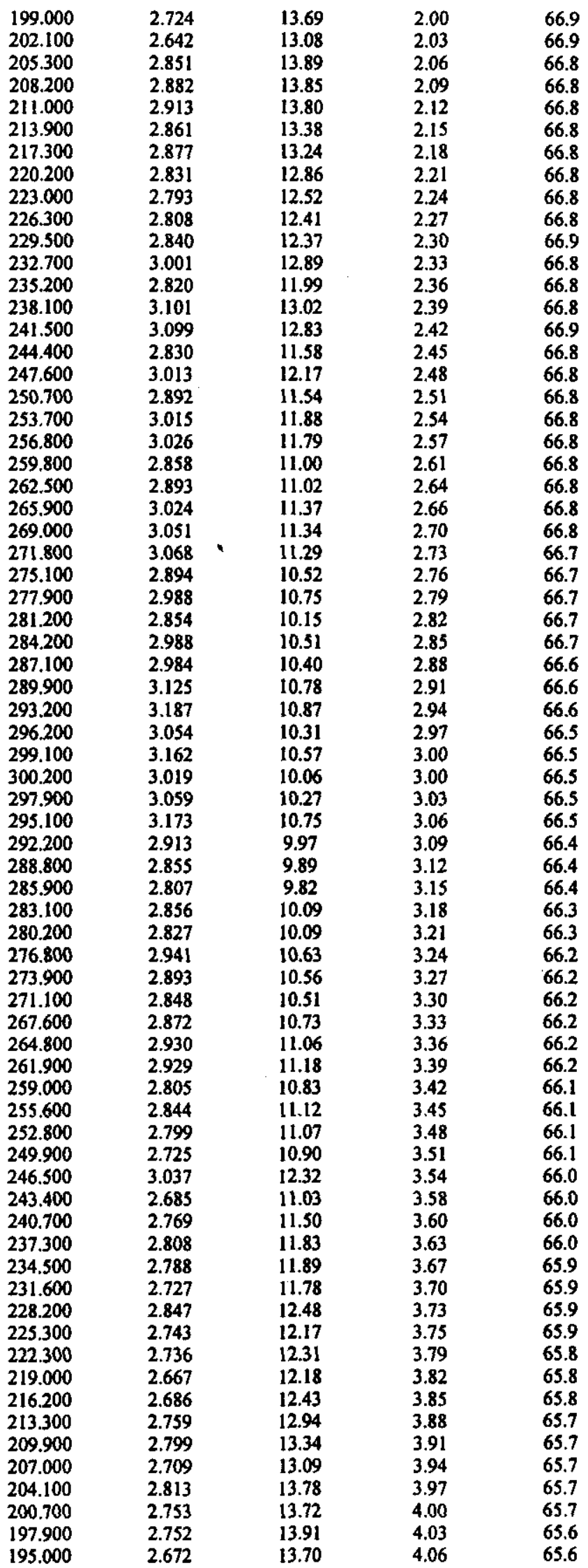


RPP-5798, Rev. 0

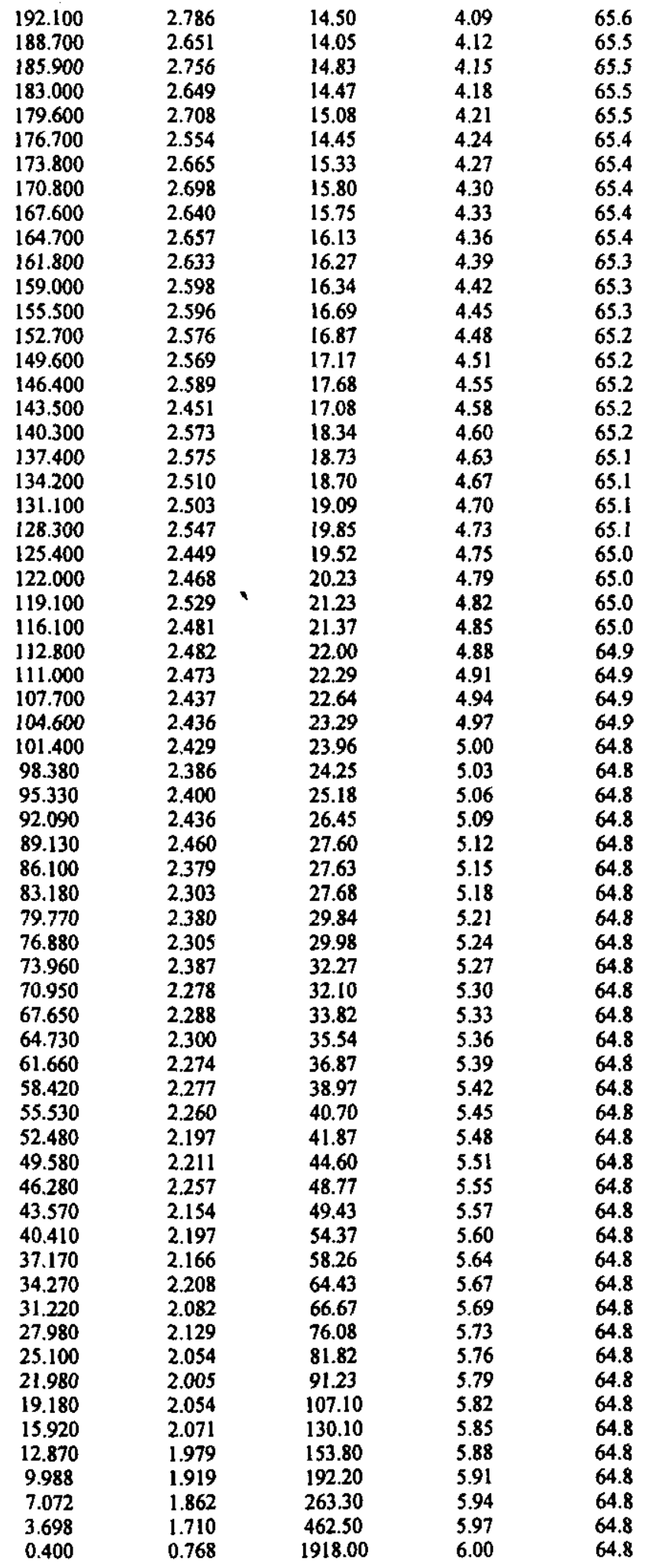


RPP-5798, Rev. 0

VISCOȘITY ANALYSIS OF

TARGET DILUTION (100 g solids/L)

AT AMBIENT TEMPERATURE 


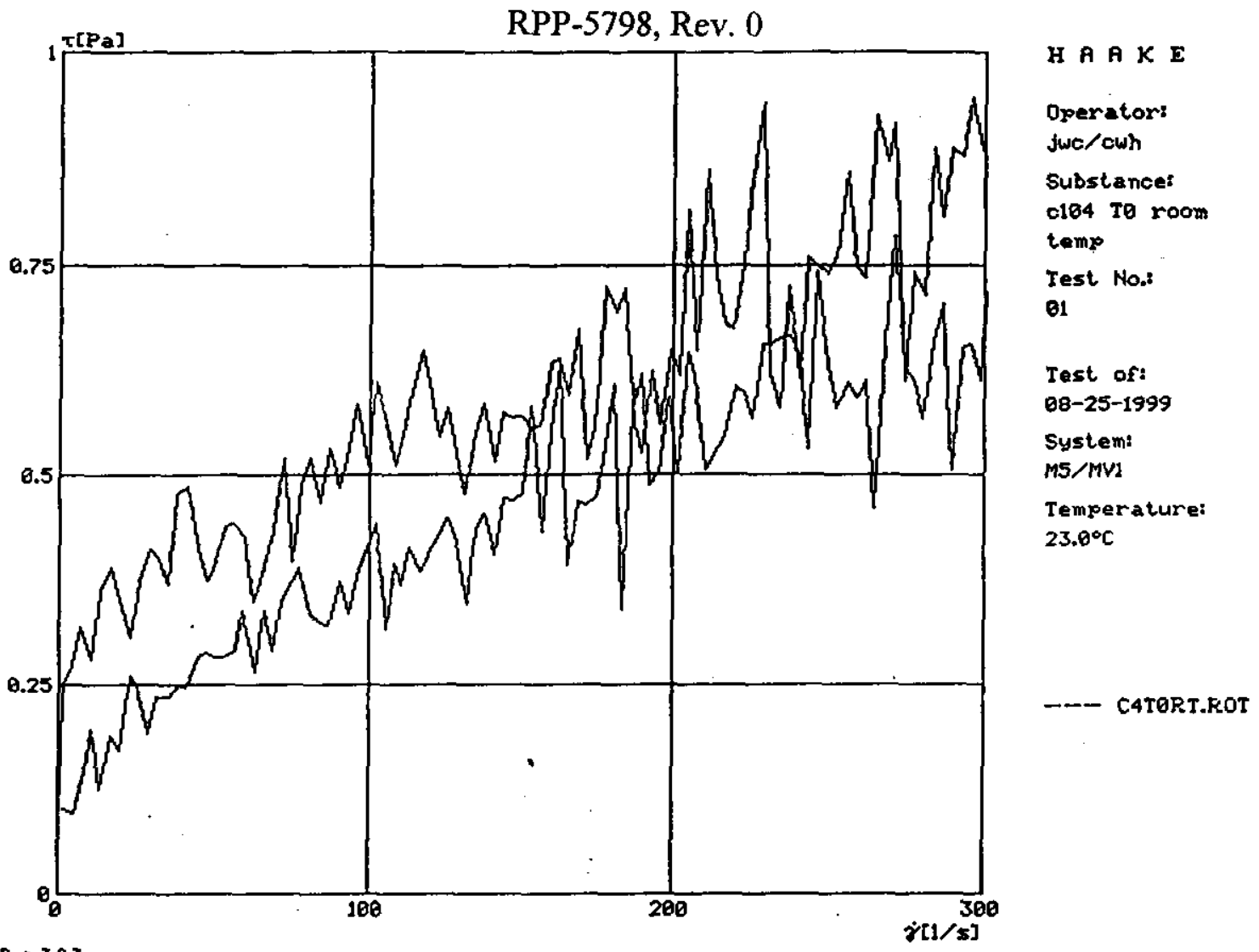

MAAKE R०ะ 3.0.3

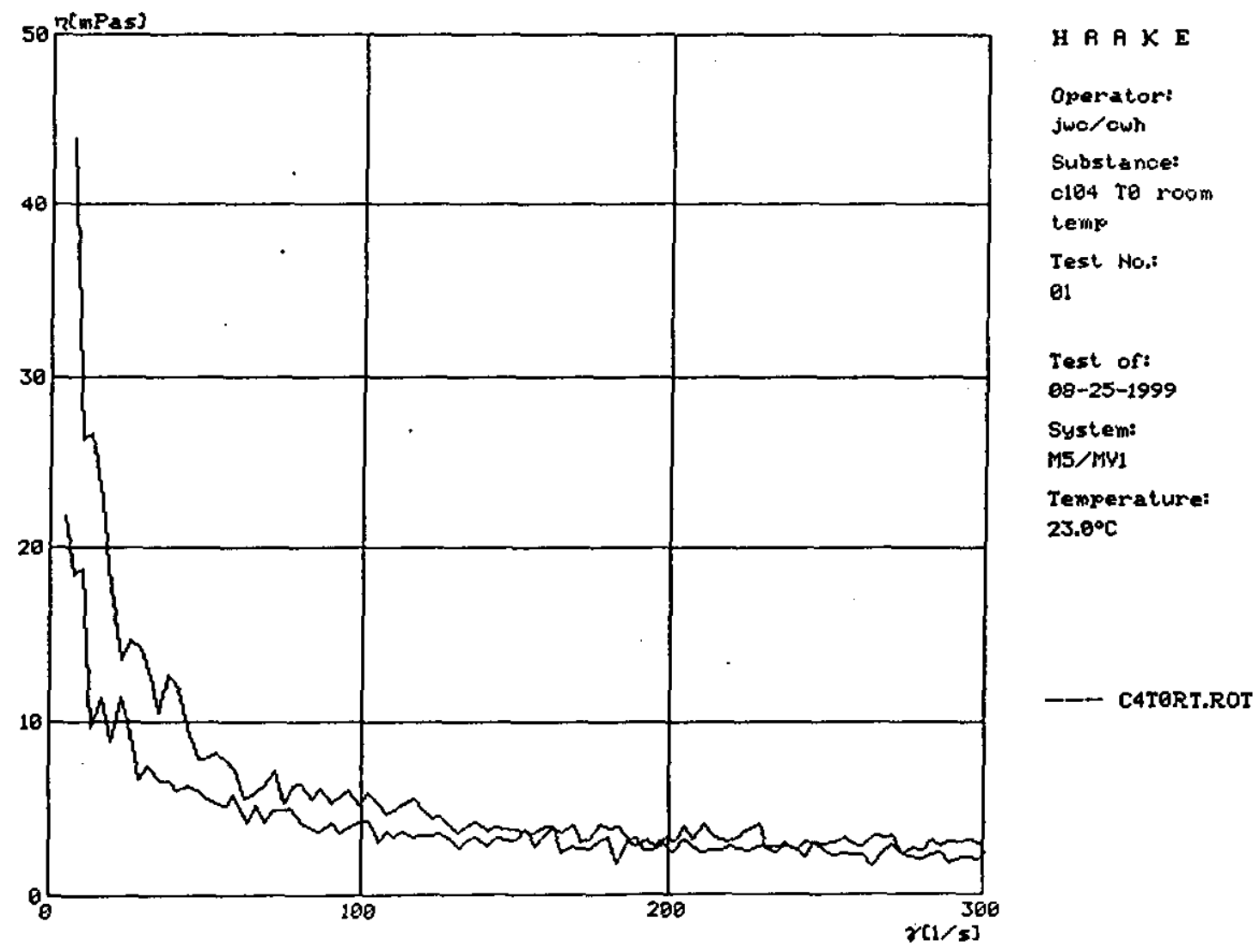


RPP-5798, Rev. 0

\begin{tabular}{|c|c|c|c|c|}
\hline$\tau$ [Pa] & $\gamma\left[s^{-1}\right]$ & $\eta[\mathrm{mPas}]$ & t[min] & $\left.\operatorname{Temp}^{\circ} \mathrm{C}\right]$ \\
\hline 0.000 & 0.077 & 0.000 & 0.00 & 30.9 \\
\hline 1.506 & 0.250 & 166.000 & 0.02 & 30.9 \\
\hline 4.460 & 0.274 & 61.380 & 0.04 & 30.9 \\
\hline 7.262 & 0.319 & 43.880 & 0.06 & 30.9 \\
\hline 10.620 & 0.279 & 26.320 & 0.08 & 30.9 \\
\hline 13.500 & 0.360 & 26.690 & 0.10 & 30.9 \\
\hline 16.950 & 0.388 & 22.880 & 0.12 & 30.9 \\
\hline 19.710 & 0.348 & 17.680 & 0.14 & 30.9 \\
\hline 22.700 & 0.306 & 13.470 & 0.16 & 30.9 \\
\hline 25.580 & 0.374 & 14.640 & 0.18 & 30.9 \\
\hline 29.030 & 0.412 & 14.200 & 0.20 & 30.9 \\
\hline 31.890 & 0.400 & 12.540 & 0.22 & 30.9 \\
\hline 34.980 & 0.367 & 10.500 & 0.24 & 30.9 \\
\hline 37.680 & 0.477 & 12.670 & 0.26 & 30.9 \\
\hline 41.100 & 0.486 & 11.820 & 0.28 & 30.9 \\
\hline 44.010 & 0.423 & 9.602 & 0.30 & 30.9 \\
\hline 47.430 & 0.374 & 7.875 & 0.32 & 30.9 \\
\hline 49.850 & 0.389 & 7.796 & 0.34 & 30.9 \\
\hline 53.220 & 0.440 & 8.269 & 0.36 & 30.9 \\
\hline 56.140 & 0.441 & 7.856 & 0.38 & 30.9 \\
\hline 59.570 & 0.426 & 7.158 & 0.40 & 30.9 \\
\hline 62.140 & 0.348 & 5.600 & 0.42 & 30.9 \\
\hline 65.320 & 0.390 & 5.963 & 0.44 & 30.9 \\
\hline 68.220 & 0.433 & 6.347 & 0.46 & 30.9 \\
\hline 71.690 & 0.519 & 7.233 & 0.48 & 30.9 \\
\hline 74.550 & 0.397 & 5.320 & 0.50 & 30.9 \\
\hline 77.500 & 0.488 & 6.294 & 0.52 & 30.9 \\
\hline 80.330 & 0.520 & 6.467 & 0.54 & 30.9 \\
\hline 83.740 & 0.467 & 5.572 & 0.56 & 30.9 \\
\hline 86.630 & 0.532 & 6.143 & 0.58 & 30.9 \\
\hline 89.990 & 0.484 & 5.373 & 0.60 & 30.9 \\
\hline 93.020 & 0.532 & 5.722 & 0.63 & 30.9 \\
\hline 95.920 & 0.586 & 6.105 & 0.64 & 30.9 \\
\hline 99.310 & 0.510 & 5.136 & 0.67 & 30.9 \\
\hline 102.200 & 0.609 & 5.960 & 0.68 & 30.9 \\
\hline 105.200 & 0.560 & 5.322 & 0.71 & 30.9 \\
\hline 108.000 & 0.510 & 4.717 & 0.73 & 30.9 \\
\hline 111.300 & 0.547 & 4.913 & 0.75 & 30.9 \\
\hline 113.600 & 0.600 & 5.279 & 0.77 & 30.9 \\
\hline 117.200 & 0.649 & 5.540 & 0.79 & 30.9 \\
\hline 119.500 & 0.610 & 5.101 & 0.81 & 30.9 \\
\hline 122.900 & 0.545 & 4.433 & 0.83 & 30.9 \\
\hline 125.800 & 0.580 & 4.606 & 0.85 & 30.9 \\
\hline 129.200 & 0.510 & 3.946 & 0.87 & 30.9 \\
\hline 131.700 & 0.476 & 3.614 & 0.89 & 30.9 \\
\hline 135.000 & 0.549 & 4.070 & 0.91 & 30.9 \\
\hline 137.800 & 0.585 & 4.242 & 0.93 & 30.9 \\
\hline 141.200 & 0.515 & 3.644 & 0.95 & 30.9 \\
\hline 144.100 & 0.576 & 3.998 & 0.97 & 30.9 \\
\hline 147.000 & 0.567 & 3.859 & 0.99 & 30.9 \\
\hline 149.800 & 0.569 & 3.798 & 1.01 & 30.9 \\
\hline 153.300 & 0.556 & 3.627 & 1.03 & 30.9 \\
\hline 156.100 & 0.558 & 3.576 & 1.05 & 30.9 \\
\hline 159.500 & 0.632 & 3.961 & 1.07 & 30.9 \\
\hline 161.800 & 0.637 & 3.937 & 1.09 & 30.9 \\
\hline 165.300 & 0.595 & 3.601 & 1.11 & 30.9 \\
\hline 168.100 & 0.673 & 4.000 & 1.13 & 30.9 \\
\hline 171.600 & 0.517 & 3.014 & 1.15 & 30.9 \\
\hline 173.800 & 0.554 & 3.187 & 1.17 & 30.9 \\
\hline 177.300 & 0.723 & 4.079 & 1.19 & 30.9 \\
\hline 180.700 & 0.694 & 3.842 & 1.21 & 30.9 \\
\hline 183.600 & 0.721 & 3.929 & 1.23 & 30.9 \\
\hline 187.000 & 0.561 & 3.000 & 1.25 & 30.9 \\
\hline 189.300 & 0.524 & 2.769 & 1.27 & 30.9 \\
\hline 192.700 & 0.623 & 3.230 & 1.29 & 30.9 \\
\hline 195.600 & 0.560 & 2.864 & 1.31 & 30.9 \\
\hline
\end{tabular}


RPP-5798, Rev. 0

\begin{tabular}{|c|c|c|c|c|}
\hline $\begin{array}{l}199.000 \\
201.700\end{array}$ & $\begin{array}{l}0.649 \\
0.619\end{array}$ & $\begin{array}{l}3.263 \\
3.068\end{array}$ & $\begin{array}{l}1.33 \\
1.35\end{array}$ & $\begin{array}{l}30.9 \\
30.9\end{array}$ \\
\hline 204.700 & 0.814 & 3.978 & 1.37 & 30.9 \\
\hline 207.600 & 0.649 & 3.126 & 1.39 & 30.9 \\
\hline 211.000 & 0.863 & 4.092 & 1.41 & 30.9 \\
\hline 213.900 & 0.736 & 3.443 & 1.43 & 30.9 \\
\hline 216.700 & 0.680 & 3.138 & 1.45 & 30.9 \\
\hline 219.600 & 0.673 & 3.063 & 1.47 & 30.9 \\
\hline 223.000 & 0.751 & 3.369 & 1.49 & 30.9 \\
\hline 225.900 & 0.855 & 3.787 & 1.51 & 30.9 \\
\hline 229.100 & 0.941 & 4.107 & 1.53 & 30.9 \\
\hline 231.600 & 0.620 & 2.675 & 1.55 & 30.9 \\
\hline 235.000 & 0.579 & 2.463 & 1.58 & 30.9 \\
\hline 237.900 & 0.725 & 3.048 & 1.59 & 30.9 \\
\hline 241.300 & 0.614 & 2.546 & 1.62 & 30.9 \\
\hline 243.600 & 0.758 & 3.112 & 1.63 & 30.9 \\
\hline 247.000 & 0.750 & 3.034 & 1.66 & 30.9 \\
\hline 249.900 & 0.740 & 2.960 & 1.67 & 30.9 \\
\hline 253.300 & 0.761 & 3.005 & 1.70 & 30.9 \\
\hline 256.200 & 0.859 & 3.352 & 1.71 & 30.9 \\
\hline 259.000 & 0.747 & 2.884 & 1.74 & 30.9 \\
\hline 261.900 & 0.734 & 2.801 & 1.75 & 30.9 \\
\hline 265.300 & 0.926 & 3.491 & 1.78 & 30.9 \\
\hline 268.800 & 0.872 & 3.246 & 1.80 & 30.9 \\
\hline 271.200 & 0.916 & 3.376 & 1.82 & 30.9 \\
\hline 274.500 & 0.614 & 2.235 & 1.84 & 30.9 \\
\hline 277.300 & 0.743 & 2.677 & 1.86 & 30.9 \\
\hline 280.800 & 0.715 & 2.545 & 1.88 & 30.9 \\
\hline 283.600 & 0.888 & 3.131 & 1.90 & 30.9 \\
\hline 286.500 & 0.808 & 2.821 & 1.92 & 30.9 \\
\hline 289.400 & 0.889 & 3.070 & 1.94 & 30.9 \\
\hline 292.800 & 0.878 & 2.997 & 1.96 & 30.9 \\
\hline 295.600 & 0.945 & 3.195 & 1.98 & 30.9 \\
\hline 298.900 & 0.885 & 2.962 & 2.00 & 30.9 \\
\hline 300.200 & 0.717 & 2.388 & 2.00 & 30.9 \\
\hline 298.500 & 0.613 & 2.054 & 2.02 & 30.9 \\
\hline 295.500 & 0.656 & 2.220 & 2.04 & 30.9 \\
\hline 292.600 & 0.651 & 2.224 & 2.06 & 30.9 \\
\hline 289.400 & 0.504 & 1.743 & 2.08 & 30.9 \\
\hline 286.900 & 0.705 & 2,456 & 2.10 & 30.9 \\
\hline 283.400 & 0.657 & 2.319 & 2.12 & 30.9 \\
\hline 280.600 & 0.568 & 2.025 & 2.14 & 30.9 \\
\hline 277.200 & 0.614 & 2.214 & 2.16 & 30.9 \\
\hline 274.500 & 0.629 & 2.292 & 2.18 & 30.9 \\
\hline 271.200 & 0.785 & 2.892 & 2.20 & 30.9 \\
\hline 268.600 & 0.656 & 2.441 & 2.22 & 30 \\
\hline 265.100 & 0.458 & 1.728 & 2.24 & 30.9 \\
\hline 262.300 & 0.613 & 2.335 & 2.26 & 30.9 \\
\hline 259.000 & 0.592 & 2.286 & 2.28 & 30.9 \\
\hline 256.600 & 0.611 & 2.382 & 2.30 & 30.9 \\
\hline 253.100 & 0.581 & 2.295 & 2.32 & 30.9 \\
\hline 250.300 & 0.631 & 2.521 & 2.34 & 30 \\
\hline 247.000 & 0.743 & 3.005 & 2.36 & 30.9 \\
\hline 244.200 & 0.530 & 2.170 & 2.38 & 30.9 \\
\hline 241.100 & 0.640 & 2.652 & 2.40 & 30.9 \\
\hline 238.100 & 0.665 & 2.793 & 2.42 & 30.9 \\
\hline 234.600 & 0.665 & 2.832 & 2.44 & 30.9 \\
\hline 232.200 & 0.656 & 2.826 & 2.46 & 30.9 \\
\hline 228.900 & 0.655 & 2.861 & 2.48 & 30 \\
\hline 226.100 & 0.567 & 2.507 & 2.50 & 30.9 \\
\hline 222.600 & 0.598 & 2.685 & 2.52 & 30.9 \\
\hline 220.000 & 0.606 & 2.755 & 2.54 & 30. \\
\hline 216.900 & 0.545 & 2.512 & 2.56 & 30.5 \\
\hline 214.300 & 0.533 & 2.488 & 2.58 & 30. \\
\hline 210.600 & 0.505 & 2.399 & 2.60 & 30.9 \\
\hline 207.200 & 0.618 & 2.981 & 2.63 & 30.9 \\
\hline 204.700 & 0.645 & 3.151 & 2.64 & 30.9 \\
\hline 201.500 & 0.500 & 2.482 & 2.67 & 30.9 \\
\hline 198.600 & 0.593 & 2.984 & 2.69 & 30.9 \\
\hline 195.200 & 0.501 & 2.564 & 2.71 & 30. \\
\hline
\end{tabular}


RPP-5798, Rev. 0

\begin{tabular}{|c|c|c|c|c|}
\hline 192.100 & 0.486 & 2.529 & 2.73 & 30.9 \\
\hline 189.500 & 0.620 & 3.273 & 2.75 & 30.9 \\
\hline 186.600 & 0.581 & 3.115 & 2.77 & 30.9 \\
\hline 183.200 & 0.338 & 1.843 & 2.79 & 30.9 \\
\hline 180.300 & 0.607 & 3.365 & 2.81 & 30.9 \\
\hline 177.300 & 0.534 & 3.010 & 2.83 & 30.9 \\
\hline 174.800 & 0.474 & 2.710 & 2.85 & 30.9 \\
\hline 171.200 & 0.464 & 2.712 & 2.87 & 30.9 \\
\hline 168.300 & 0.470 & 2.792 & 2.89 & 30.9 \\
\hline 164.900 & 0.391 & 2.371 & 2.91 & 30.9 \\
\hline 162.400 & 0.635 & 3.911 & 2.93 & 30.9 \\
\hline 159.200 & 0.549 & 3.448 & 2.95 & 30.9 \\
\hline 156.300 & 0.433 & 2.767 & 2.97 & 30.9 \\
\hline 152.900 & 0.583 & 3.812 & 2.99 & 30.9 \\
\hline 150.000 & 0.476 & 3.173 & 3.01 & 30.9 \\
\hline 147.200 & 0.468 & 3.183 & 3.03 & 30.9 \\
\hline 144.100 & 0.471 & 3.270 & 3.05 & 30.9 \\
\hline 140.900 & 0.403 & 2.859 & 3.07 & 30.9 \\
\hline 138.000 & 0.454 & 3.288 & 3.09 & 30.9 \\
\hline 135.000 & 0.436 & 3.233 & 3.11 & 30.9 \\
\hline 132.100 & 0.346 & 2.616 & 3.13 & 30.9 \\
\hline 128.700 & 0.419 & 3.255 & 3.15 & 30.9 \\
\hline 125.800 & 0.449 & 3.566 & 3.17 & 30.9 \\
\hline 122.400 & 0.425 & 3.477 & 3.19 & 30.9 \\
\hline 119.500 & 0.403 & 3.370 & 3.21 & 30.8 \\
\hline 116.700 & 0.385 & 3.299 & 3.23 & 30.9 \\
\hline 113.200 & 0.414 & 3.653 & 3.25 & 30.9 \\
\hline 110.400 & 0.370 & 3.350 & 3.27 & 30.9 \\
\hline 108.100 & 0.394 & 3.643 & 3.29 & 30.9 \\
\hline 105.300 & 0.315 & 2.994 & 3.31 & 30.9 \\
\hline 101.900 & 0.441 & 4.326 & 3.33 & 30.9 \\
\hline 99.020 & 0.415 & 4.187 & 3.35 & 30.9 \\
\hline 95.590 & 0.378 & 3.956 & 3.37 & 30.9 \\
\hline 93.150 & 0.332 & 3.568 & 3.39 & 30.8 \\
\hline 89.780 & 0.374 & 4.170 & 3.41 & 30.9 \\
\hline 86.880 & 0.320 & 3.679 & 3.43 & 30.9 \\
\hline 83.570 & 0.323 & 3.870 & 3.45 & 30.9 \\
\hline 80.550 & 0.333 & 4.138 & 3.47 & 30.9 \\
\hline 76.860 & 0.388 & 5.050 & 3.49 & 30.9 \\
\hline 74.720 & 0.373 & 4.992 & 3.51 & 30.9 \\
\hline 71.440 & 0.352 & 4.923 & 3.53 & 30.9 \\
\hline 68.470 & 0.290 & 4.233 & 3.55 & 30.9 \\
\hline 65.380 & 0.337 & 5.156 & 3.58 & 30.9 \\
\hline 63.070 & 0.265 & 4.206 & 3.59 & 30.9 \\
\hline 58.500 & 0.339 & 5.795 & 3.62 & 30.9 \\
\hline 56.540 & 0.291 & 5.152 & 3.63 & 30.8 \\
\hline 52.720 & 0.283 & 5.372 & 3.65 & 30.9 \\
\hline 49.850 & 0.282 & 5.660 & 3.67 & 30.9 \\
\hline 47.710 & 0.289 & 6.055 & 3.69 & 30.9 \\
\hline 44.530 & 0.283 & 6.361 & 3.71 & 30.9 \\
\hline 40.490 & 0.245 & 6.041 & 3.74 & 30.9 \\
\hline 38.090 & 0.250 & 6.573 & 3.75 & 30.9 \\
\hline 35.440 & 0.234 & 6.606 & 3.77 & 30.9 \\
\hline 31.660 & 0.236 & 7.446 & 3.80 & 30.9 \\
\hline 28.690 & 0.193 & 6.721 & 3.82 & 30.9 \\
\hline 25.220 & 0.247 & 9.811 & 3.84 & 30.8 \\
\hline 22.820 & 0.260 & 11.400 & 3.86 & 30.8 \\
\hline 19.460 & 0.173 & 8.867 & 3.88 & 30.9 \\
\hline 16.600 & 0.190 & 11.420 & 3.90 & 30.8 \\
\hline 13.130 & 0.127 & 9.629 & 3.92 & 30.8 \\
\hline 10.430 & 0.196 & 18.790 & 3.94 & 30.9 \\
\hline 7.396 & 0.136 & 18.440 & 3.96 & 30.9 \\
\hline 4.518 & 0.099 & 21.990 & 3.98 & 30.9 \\
\hline 1.125 & 0.104 & 92.200 & 4.00 & 30.8 \\
\hline
\end{tabular}


RPP-5798, Rev. 0

\section{VISCOṠITY ANALYSIS OF}

TARGET DILUTION (100 g solids/L) AT $45^{\circ} \mathrm{C}$ 


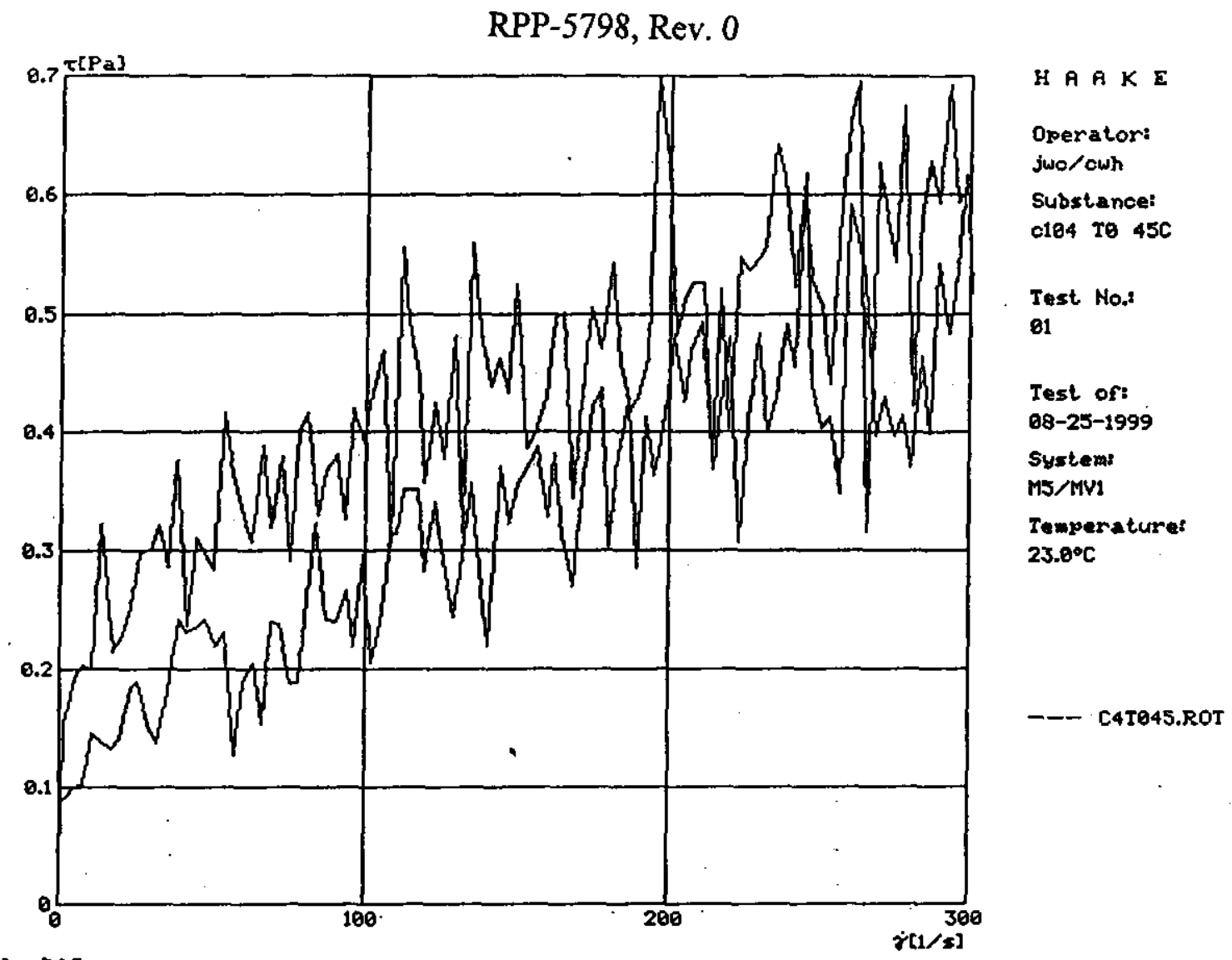

HARKE ROt $\mathbf{3 . 0 . 3}$

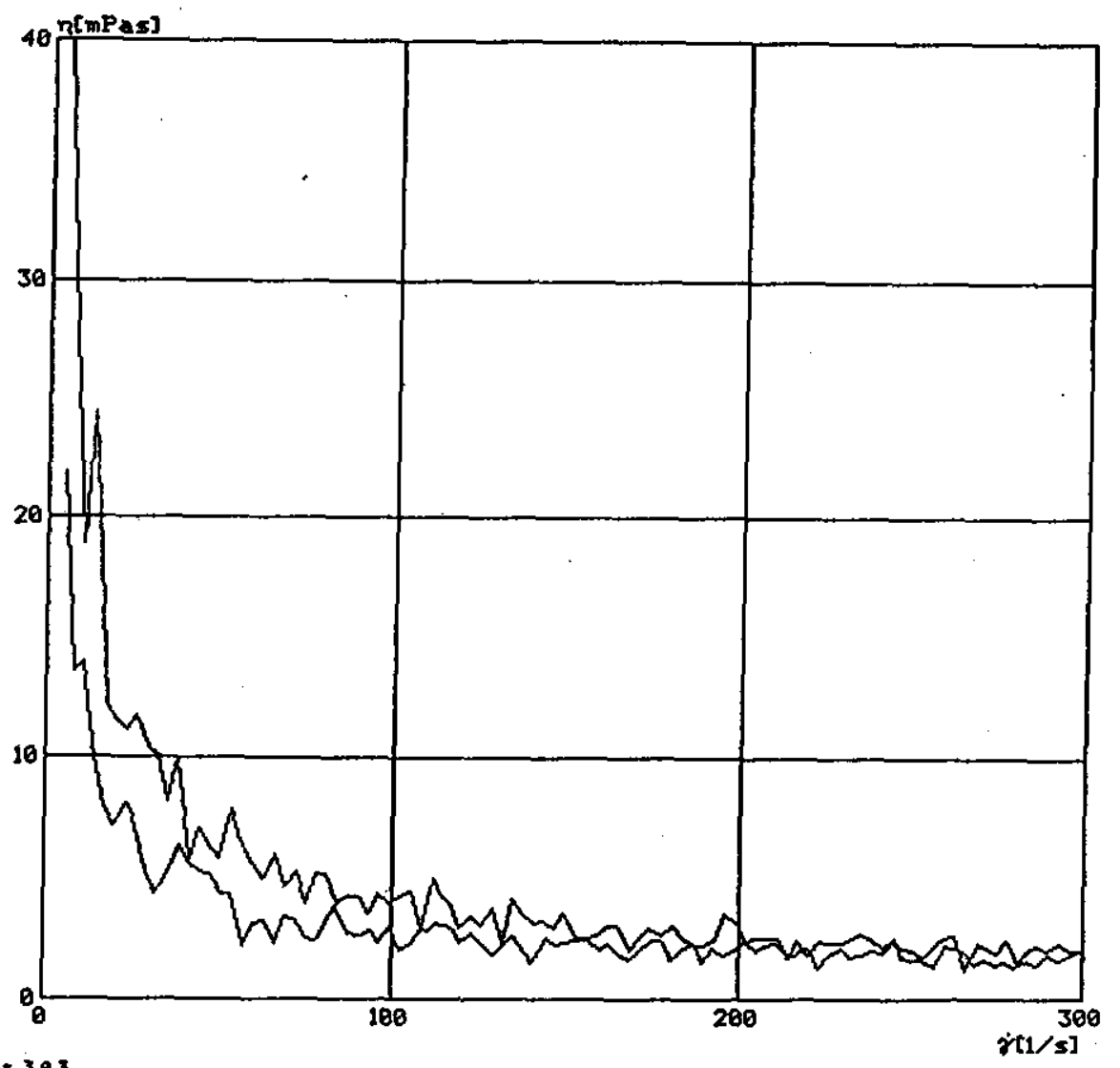

Hคค

Operator:

jwo/cwh

Substance:

0104 TO $45 \mathrm{C}$

Test No.:

01

Test of:

08-25-1999

System:

M5/MV1

Temperature:

$23.9^{\circ} \mathrm{C}$ 
RPP-5798, Rev. 0

\begin{tabular}{|c|c|c|c|c|}
\hline$\tau[\mathbf{P a}]$ & $\gamma\left[\mathbf{s}^{-1}\right]$ & $\eta$ [mPas] & $t[\mathrm{~min}]$ & $\left.\left.\operatorname{Temp}\right|^{\circ} \mathrm{C}\right]$ \\
\hline 0.000 & 0.028 & 0.000 & 0.00 & 45.0 \\
\hline 1.468 & 0.152 & 103.300 & 0.02 & 44.9 \\
\hline 4.842 & 0.194 & 39.970 & 0.04 & 45.0 \\
\hline 7.301 & 0.203 & 27.830 & 0.06 & 45.0 \\
\hline 10.620 & 0.200 & 18.830 & 0.08 & 45.0 \\
\hline 13.270 & 0.324 & 24.410 & 0.10 & 45.0 \\
\hline 17.630 & 0.214 & 12.110 & 0.12 & 45.0 \\
\hline 20.030 & 0.230 & 11.490 & 0.14 & 45.0 \\
\hline 22.680 & 0.253 & 11.150 & 0.16 & 45.0 \\
\hline 25.500 & 0.299 & 11.730 & 0.18 & 45.0 \\
\hline 28.970 & 0.301 & 10.380 & 0.20 & 45.0 \\
\hline 31.890 & 0.321 & 10.060 & 0.22 & 45.0 \\
\hline 34.980 & 0.286 & 8.177 & 0.24 & 45.0 \\
\hline 37.400 & 0.375 & 10.040 & 0.26 & 45.0 \\
\hline 41.500 & 0.238 & 5.723 & 0.28 & 45.0 \\
\hline 44.110 & 0.310 & 7.030 & 0.30 & 45.0 \\
\hline 47.350 & 0.294 & 6.210 & 0.32 & 45.0 \\
\hline 49.790 & 0.285 & 5.725 & 0.34 & 45.0 \\
\hline 53.240 & 0.417 & 7.839 & 0.36 & 45.0 \\
\hline 56.100 & 0.363 & 6.471 & 0.38 & 45.0 \\
\hline 59.490 & 0.329 & 5.522 & 0.40 & 45.0 \\
\hline 62.140 & 0.308 & 4.952 & 0.42 & 45.0 \\
\hline 65.320 & 0.388 & 5.933 & 0.44 & 44.9 \\
\hline 68.180 & 0.319 & 4.680 & 0.46 & 45.0 \\
\hline 71.650 & 0.379 & 5.291 & 0.48 & 45.0 \\
\hline 74.530 & 0.292 & 3.920 & 0.50 & 45.0 \\
\hline 77.540 & 0.403 & 5.199 & 0.52 & 45.0 \\
\hline 80.330 & 0.416 & 5.178 & 0.54 & 45.0 \\
\hline 83.720 & 0.330 & 3.936 & 0.56 & 44.9 \\
\hline 86.630 & 0.368 & 4.245 & 0.58 & 45.0 \\
\hline 89.970 & 0.381 & 4.234 & 0.60 & 45.0 \\
\hline 92.980 & 0.328 & 3.523 & 0.63 & 45.0 \\
\hline 95.880 & 0.420 & 4.378 & 0.64 & 45.0 \\
\hline 99.330 & 0.390 & 3.921 & 0.67 & 45.0 \\
\hline 102.200 & 0.437 & 4.271 & 0.68 & 45.0 \\
\hline 105.300 & 0.470 & 4.463 & 0.71 & 45.0 \\
\hline 108.000 & 0.304 & 2.811 & 0.73 & 44.9 \\
\hline 111.500 & 0.556 & 4.988 & 0.75 & 45.0 \\
\hline 113.600 & 0.494 & 4.352 & 0.77 & 45.0 \\
\hline 117.200 & 0.449 & 3.826 & 0.79 & 45.0 \\
\hline 119.500 & 0.357 & 2.990 & 0.81 & 45.0 \\
\hline 122.900 & 0.425 & 3.460 & 0.83 & 45.0 \\
\hline 125.800 & 0.377 & 2.998 & 0.85 & 45.0 \\
\hline 129.200 & 0.482 & 3.730 & 0.87 & 45.0 \\
\hline 131.900 & 0.315 & 2.390 & 0.89 & 45.0 \\
\hline 134.600 & 0.559 & 4.155 & 0.91 & 45.0 \\
\hline 137.600 & 0.486 & 3.534 & 0.93 & 45.0 \\
\hline 141.200 & 0.440 & 3.115 & 0.95 & 45.0 \\
\hline 144.100 & 0.463 & 3.211 & 0.97 & 45.0 \\
\hline 147.000 & 0.435 & 2.959 & 0.99 & 45.0 \\
\hline 149.800 & 0.525 & 3.501 & 1.01 & 45.0 \\
\hline 153.300 & 0.386 & 2.520 & 1.03 & 45.0 \\
\hline 156.100 & 0.399 & 2.558 & 1.05 & 45.0 \\
\hline 159.500 & 0.430 & 2.693 & 1.07 & 45.0 \\
\hline 161.800 & 0.497 & 3.069 & 1.09 & 45.0 \\
\hline 165.300 & 0.502 & 3.037 & 1.11 & 45.0 \\
\hline 168.100 & 0.345 & 2.052 & 1.13 & 45.0 \\
\hline 171.200 & 0.430 & 2.513 & 1.15 & 45.0 \\
\hline 173.800 & 0.505 & 2.906 & 1.17 & 45.0 \\
\hline 177.300 & 0.471 & 2.655 & 1.19 & 45.0 \\
\hline 180.700 & 0.542 & 2.997 & 1.21 & 45.0 \\
\hline 183.600 & 0.460 & 2.505 & 1.23 & 45.0 \\
\hline 187.000 & 0.419 & 2.239 & 1.25 & 45.0 \\
\hline 189.300 & 0.430 & 2.272 & 1.27 & 45.0 \\
\hline 192.700 & 0.463 & 2.403 & 1.29 & 45.0 \\
\hline 195.600 & 0.699 & 3.571 & 1.31 & 45.0 \\
\hline
\end{tabular}


RPP-5798, Rev. 0

\begin{tabular}{|c|c|c|c|c|}
\hline $\begin{array}{l}199.000 \\
201.700\end{array}$ & $\begin{array}{l}0.630 \\
0.484\end{array}$ & $\begin{array}{l}3.168 \\
2.402\end{array}$ & $\begin{array}{l}1.33 \\
1.35\end{array}$ & $\begin{array}{l}45.0 \\
45.0\end{array}$ \\
\hline 204.700 & 0.513 & 2.507 & 1.37 & 45.0 \\
\hline 207.600 & 0.526 & 2.534 & 1.39 & 45.0 \\
\hline 211.000 & 0.527 & 2.495 & 1.41 & 45.0 \\
\hline 213.900 & 0.375 & 1.755 & 1.43 & 45.0 \\
\hline 216.700 & 0.521 & 2.405 & 1.45 & 45.0 \\
\hline 219.600 & 0.404 & 1.838 & 1.47 & 45.0 \\
\hline 223.000 & 0.546 & 2.450 & 1.49 & 45,0 \\
\hline 225.900 & 0.536 & 2.373 & 1.51 & 45.0 \\
\hline 229.100 & 0.547 & 2.387 & 1.53 & 45.0 \\
\hline 231.600 & 0.557 & 2.404 & 1.55 & 45,0 \\
\hline 235.000 & 0.643 & 2.735 & 1.58 & 45.0 \\
\hline 237.900 & 0.615 & 2.587 & 1.59 & 45.0 \\
\hline 241.300 & 0.523 & 2.168 & 1.62 & 45,0 \\
\hline 243.600 & 0.606 & 2.489 & 1.63 & 45.0 \\
\hline 247.000 & 0.529 & 2.143 & 1.66 & 45.0 \\
\hline 249.900 & 0.506 & 2.026 & 1.67 & 45.0 \\
\hline 253.300 & 0.441 & 1.743 & 1.70 & 45.0 \\
\hline 256.200 & 0.587 & 2.291 & 1.71 & 45.0 \\
\hline 259.000 & 0.661 & 2.552 & 1.74 & 45.0 \\
\hline 261.900 & 0.696 & 2.656 & 1.75 & 45.0 \\
\hline 265.300 & 0.316 & 1.190 & 1.78 & 45.0 \\
\hline 268.800 & 0.626 & 2.330 & 1.80 & 45.0 \\
\hline 271.200 & 0.592 & 2.181 & 1.82 & 45.0 \\
\hline 274.500 & 0.544 & 1.984 & 1.84 & 45.0 \\
\hline 277.300 & 0.676 & 2.437 & 1.86 & 45.0 \\
\hline 280.800 & 0.424 & 1.512 & 1.88 & 45.0 \\
\hline 283.600 & 0.587 & 2.069 & 1.90 & 45.0 \\
\hline 286.500 & 0.628 & 2.192 & 1.92 & 45.0 \\
\hline 289.400 & 0.593 & 2.050 & 1.94 & 45.0 \\
\hline 292.800 & 0.693 & 2.368 & 1.96 & 45.0 \\
\hline 295.600 & 0.594 & 2.008 & 1.98 & 45.0 \\
\hline 298.900 & 0.608 & 2.033 & 2.00 & 45.0 \\
\hline 300.200 & 0.518 & 1.725 & 2.00 & 45.0 \\
\hline 298.500 & 0.616 & 2.063 & 2.02 & 45.0 \\
\hline 295.300 & 0.518 & 1.754 & 2.04 & 45.0 \\
\hline 292.600 & 0.483 & 1.651 & 2.06 & 45.0 \\
\hline 289.400 & 0.542 & 1.872 & 2.08 & 45.0 \\
\hline 286.700 & 0.400 & 1.395 & 2.10 & 45.0 \\
\hline 283.400 & 0.464 & 1.637 & 2.12 & 45.0 \\
\hline 280.400 & 0.371 & 1.322 & 2.14 & 45.0 \\
\hline 277.200 & 0.415 & 1.496 & 2.16 & 45.0 \\
\hline 274.500 & 0.398 & 1.450 & 2.18 & 45.0 \\
\hline 271.400 & 0.431 & 1.586 & 2.20 & 45.0 \\
\hline 268.600 & 0.398 & 1.482 & 2.22 & 45.0 \\
\hline 265.100 & 0.528 & 1.993 & 2.24 & 45.0 \\
\hline 262.300 & 0.564 & 2.150 & 2.26 & 45.0 \\
\hline 259.200 & 0.591 & 2.279 & 2.28 & 45.0 \\
\hline 256.600 & 0.348 & 1.358 & 2.30 & 45.0 \\
\hline 253.100 & 0.413 & 1.630 & 2.32 & 45.0 \\
\hline 250.300 & 0.405 & 1.617 & 2.34 & 45.0 \\
\hline 247.000 & 0.441 & 1.787 & 2.36 & 45.0 \\
\hline 244.400 & 0.619 & 2.532 & 2.38 & 45.0 \\
\hline 240.900 & 0.456 & 1.891 & 2.40 & 45.0 \\
\hline 238.100 & 0.492 & 2.065 & 2.42 & 45.0 \\
\hline 234.800 & 0.429 & 1.827 & 2.44 & 45.0 \\
\hline 232.200 & 0.401 & 1.726 & 2.46 & 45.0 \\
\hline 228.900 & 0.484 & 2.116 & 2.48 & 45.0 \\
\hline 226.100 & 0.410 & 1.815 & 2.50 & 45.0 \\
\hline 222.600 & 0.307 & 1.378 & 2.52 & 45.0 \\
\hline 219.800 & 0.480 & 2.185 & 2.54 & 45.0 \\
\hline 216.900 & 0.424 & 1.952 & 2.56 & 45.0 \\
\hline 214.300 & 0.368 & 1.719 & 2.58 & 45.0 \\
\hline 210.600 & 0.493 & 2.338 & 2.60 & 45.0 \\
\hline 207.200 & 0.469 & 2.265 & 2.63 & 45.0 \\
\hline 204.700 & 0.427 & 2.087 & 2.64 & 45.0 \\
\hline 201.500 & 0.482 & 2.393 & 2.67 & 45.0 \\
\hline 198.600 & 0.415 & 2.087 & 2.69 & 45.0 \\
\hline 195.200 & 0.364 & 1.865 & 2.71 & 45.0 \\
\hline
\end{tabular}


RPP-5798, Rev. 0

\begin{tabular}{|c|c|c|c|c|}
\hline 192.300 & 0.414 & 2.150 & 2.73 & 45.0 \\
\hline 189.300 & 0.287 & 1.516 & 2.75 & 45.0 \\
\hline 186.600 & 0.428 & 2.292 & 2.77 & 45.0 \\
\hline 183.200 & 0.381 & 2.082 & 2.79 & 45.0 \\
\hline 180.500 & 0.304 & 1.686 & 2.81 & 45.0 \\
\hline 177.300 & 0.439 & 2.474 & 2.83 & 45.0 \\
\hline 174.600 & 0.423 & 2.423 & 2.85 & 45.0 \\
\hline 171.000 & 0.346 & 2.024 & 2.87 & 45.0 \\
\hline 168.100 & 0.270 & 1.606 & 2.89 & 45.0 \\
\hline 164.900 & 0.313 & 1.895 & 2.91 & 45.0 \\
\hline 162.400 & 0.381 & 2.346 & 2.93 & 45.0 \\
\hline 159.700 & 0.328 & 2.054 & 2.94 & 45.0 \\
\hline 156.500 & 0.389 & 2.483 & 2.96 & 45.0 \\
\hline 153.300 & 0.367 & 2.396 & 2.99 & 45.0 \\
\hline 150.000 & 0.357 & 2.379 & 3.01 & 45.0 \\
\hline 147.200 & 0.322 & 2.191 & 3.03 & 45.0 \\
\hline 144.700 & 0.371 & 2.565 & 3.04 & 45.0 \\
\hline 140.700 & 0.220 & 1.562 & 3.07 & 45.0 \\
\hline 138.400 & 0.266 & 1.923 & 3.08 & 45.0 \\
\hline 135.000 & 0.356 & 2.637 & 3.11 & 45.0 \\
\hline 132.300 & 0.296 & 2.241 & 3.13 & 45.0 \\
\hline 129.200 & 0.243 & 1.883 & 3.15 & 45.0 \\
\hline 126.000 & 0.291 & 2.311 & 3.16 & 45.0 \\
\hline 122.900 & 0.341 & 2.776 & 3.19 & 45.0 \\
\hline 119.500 & 0.282 & 2.357 & 3.21 & 45.0 \\
\hline 117.200 & 0.351 & 2.996 & 3.22 & 45.0 \\
\hline 112.700 & 0.352 & 3.122 & 3.25 & 45.0 \\
\hline 110.500 & 0.314 & 2.840 & 3.27 & 45.0 \\
\hline 108.300 & 0.314 & 2.901 & 3.29 & 45.0 \\
\hline 105.500 & 0.249 & 2.356 & 3.31 & 45.0 \\
\hline 102.100 & 0.206 & 2.013 & 3.33 & 45.0 \\
\hline 99.080 & 0.297 & 3.001 & 3.35 & 45.0 \\
\hline 95.710 & 0.220 & 2.295 & 3.37 & 45.0 \\
\hline 93.290 & 0.268 & 2.868 & 3.39 & 45.0 \\
\hline 90.100 & 0.240 & 2.668 & 3.41 & 45.0 \\
\hline 86.880 & 0.242 & 2.785 & 3.43 & 45.0 \\
\hline 82.960 & 0.323 & 3.898 & 3.45 & 45.0 \\
\hline 80.590 & 0.261 & 3.238 & 3.47 & 45.0 \\
\hline 77.620 & 0.188 & 2.421 & 3.49 & 45.0 \\
\hline 74.780 & 0.188 & 2.514 & 3.51 & 45.0 \\
\hline 71.310 & 0.238 & 3.335 & 3.53 & 45.0 \\
\hline 68.470 & 0.239 & 3.497 & 3.55 & 45.0 \\
\hline 65.340 & 0.152 & 2.328 & 3.58 & 45.0 \\
\hline 62.660 & 0.206 & 3.286 & 3.59 & 45.0 \\
\hline 59.220 & 0.189 & 3.185 & 3.62 & 45.0 \\
\hline 56.330 & 0.126 & 2.232 & 3.63 & 45.0 \\
\hline 52.860 & 0.232 & 4.388 & 3.66 & 44.9 \\
\hline 50.490 & 0.219 & 4.345 & 3.67 & 45.0 \\
\hline 47.120 & 0.242 & 5.133 & 3.70 & 45.0 \\
\hline 44.220 & 0.234 & 5.295 & 3.71 & 45.0 \\
\hline 40.770 & 0.232 & 5.691 & 3.74 & 45.0 \\
\hline 38.140 & 0.243 & 6.360 & 3.75 & 45.0 \\
\hline 34.980 & 0.180 & 5.138 & 3.78 & 45.0 \\
\hline 31.570 & 0.136 & 4.317 & 3.80 & 45.0 \\
\hline 28.710 & 0.152 & 5.295 & 3.82 & 45.0 \\
\hline 25.240 & 0.190 & 7.521 & 3.84 & 45.0 \\
\hline 22.800 & 0.185 & 8.100 & 3.86 & 44.9 \\
\hline 19.460 & 0.139 & 7.143 & 3.88 & 45.0 \\
\hline 16.620 & 0.132 & 7.921 & 3.90 & 45.0 \\
\hline 13.170 & 0.139 & 10.530 & 3.92 & 45.0 \\
\hline 10.410 & 0.145 & 13.930 & 3.94 & 45.0 \\
\hline 7.396 & 0.100 & 13.560 & 3.96 & 45.0 \\
\hline 4.556 & 0.100 & 21.930 & 3.98 & 45.0 \\
\hline 1.144 & 0.088 & 77.060 & 4.00 & 45.0 \\
\hline
\end{tabular}


RPP-5798, Rev. 0

\section{VISCOSITY ANALYSIS OF}

TARGET DILUTION (100 g solids/L) AT $65^{\circ} \mathrm{C}$ 


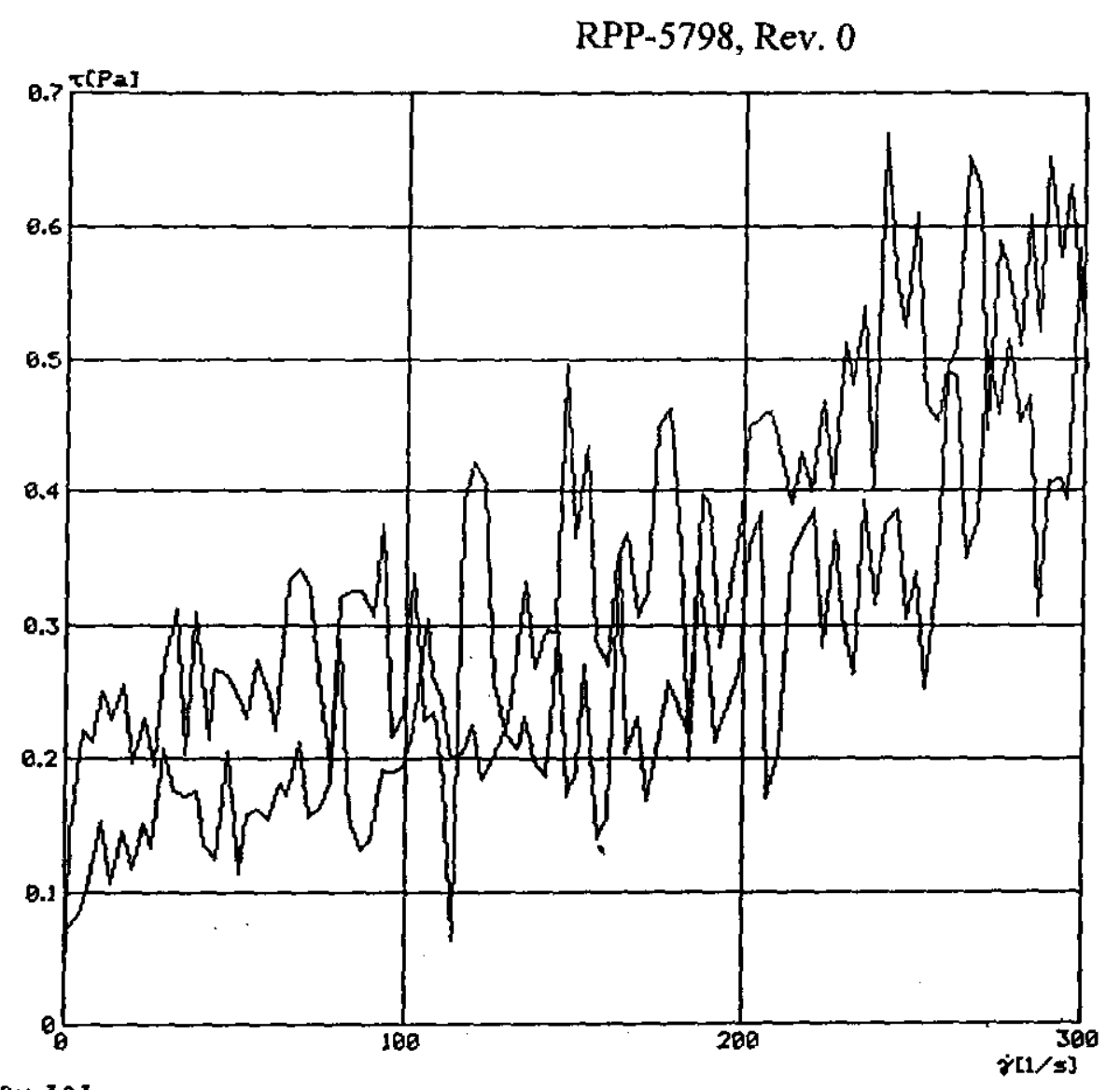

H A A $X E$

Operator:

jwo/cwh

Substance:

0154 TO $65 \mathrm{C}$

Test No:

61

Test of:

08-25-1999

System:

M5/MU1

Temperature:

$23.0^{\circ} \mathrm{C}$

C4T065.ROT

HQAKF RAT 3 A 3

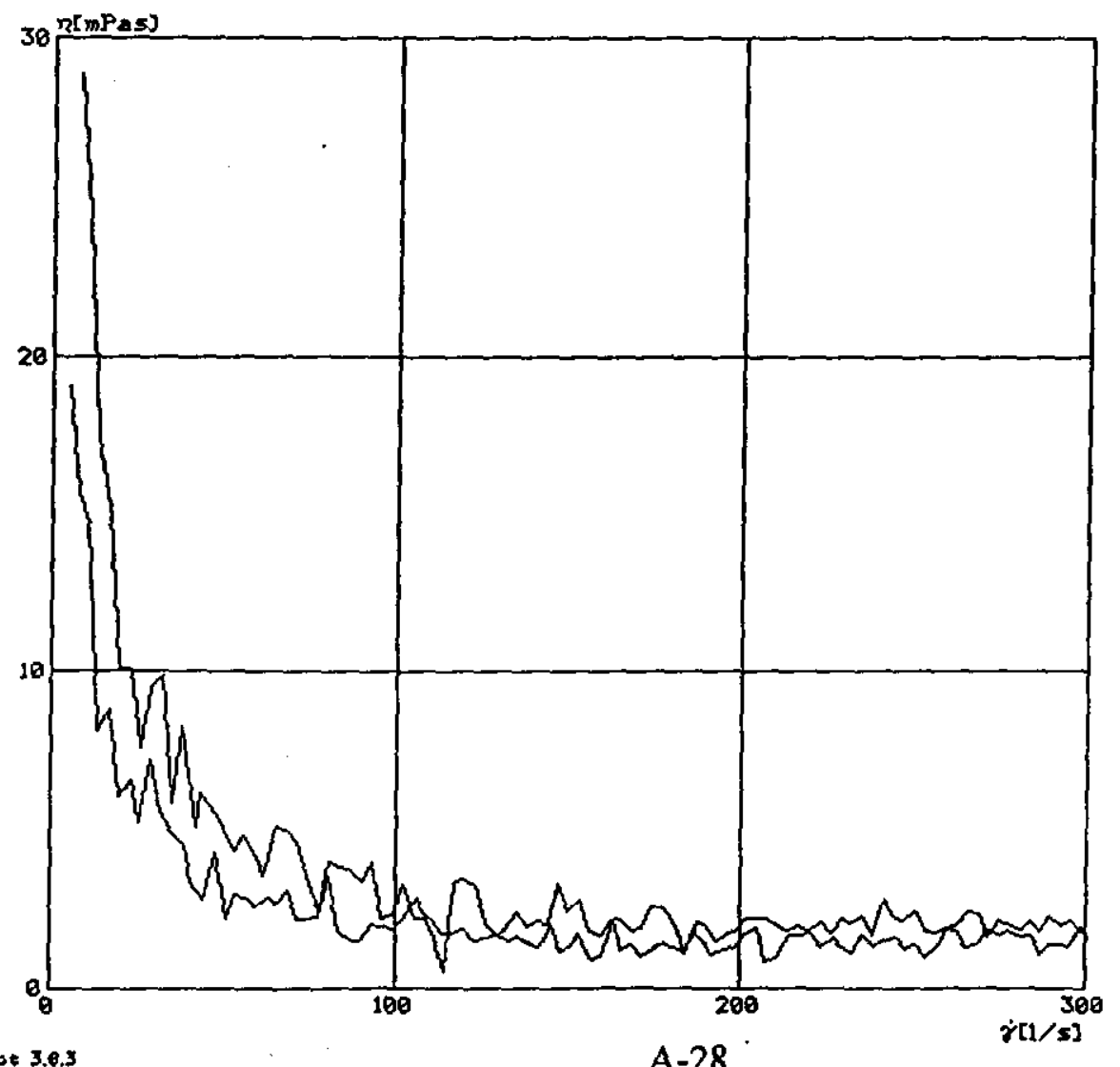

H ค ค $\mathrm{K} \mathrm{E}$

Operator:

jue/owh

Substances

0184 TO 658

Test No.2

os

Test of:

08-25-1999

System:

M5/MVI

Temperature:

$23.0^{\circ} \mathrm{C}$

D- C4TO65.ROT 
RPP-5798, Rev. 0

\begin{tabular}{|c|c|c|c|c|}
\hline$\tau[\mathbf{P a}]$ & $\gamma\left[\mathbf{s}^{-1}\right]$ & $\eta[m P a s]$ & $t[\mathrm{~min}]$ & $\left.\operatorname{Temp} \mid{ }^{\circ} \mathrm{C}\right]$ \\
\hline 0.000 & 0.026 & 0.000 & 0.00 & 64.7 \\
\hline 1.525 & 0.141 & 92.360 & 0.02 & 64.7 \\
\hline 4.842 & 0.221 & 45.660 & 0.04 & 64.7 \\
\hline 7.320 & 0.212 & 28.900 & 0.06 & 64.7 \\
\hline 10.290 & 0.250 & 24.320 & 0.08 & 64.7 \\
\hline 13.210 & 0.231 & 17.450 & 0.10 & 64.7 \\
\hline 16.930 & 0.257 & 15.150 & 0.12 & 64.7 \\
\hline 19.520 & 0.197 & 10.090 & 0.14 & 64.7 \\
\hline 22.850 & 0.230 & 10.070 & 0.16 & 64.7 \\
\hline 25.680 & 0.194 & 7.543 & 0.18 & 64.8 \\
\hline 28.780 & 0.274 & 9.503 & 0.20 & 64.7 \\
\hline 31.870 & 0.313 & 9.827 & 0.22 & 64.7 \\
\hline 34.830 & 0.204 & 5.851 & 0.24 & 64.7 \\
\hline 37.630 & 0.311 & 8.273 & 0.26 & 64.8 \\
\hline 41.780 & 0.214 & 5.116 & 0.28 & 64.8 \\
\hline 43.610 & 0.266 & 6.109 & 0.30 & 64.8 \\
\hline 47.390 & 0.263 & 5.552 & 0.32 & 64.8 \\
\hline 49.580 & 0.251 & 5.059 & 0.34 & 64.8 \\
\hline 53.180 & 0.230 & 4.317 & 0.36 & 64.8 \\
\hline 56.040 & 0.274 & 4.881 & 0.38 & 64.7 \\
\hline 59.320 & 0.250 & 4.213 & 0.40 & 64.7 \\
\hline 61.660 & 0.221 & 3.579 & 0.42 & 64.8 \\
\hline 65.250 & 0.333 & 5.104 & 0.44 & 64.8 \\
\hline 68.180 & 0.342 & 5.016 & 0.46 & 64.8 \\
\hline 71.160 & 0.331 & 4.647 & 0.48 & 64.8 \\
\hline 74.420 & 0.248 & 3.333 & 0.50 & 64.8 \\
\hline 77.450 & 0.195 & 2.519 & 0.52 & 64.7 \\
\hline 80.170 & 0.324 & 4.042 & 0.54 & 64.8 \\
\hline 83.740 & 0.327 & 3.904 & 0.56 & 64.8 \\
\hline 86.620 & 0.327 & 3.780 & 0.58 & 64.8 \\
\hline 89.970 & 0.308 & 3.423 & 0.60 & 64.8 \\
\hline 93.000 & 0.377 & 4.048 & 0.63 & 64.8 \\
\hline 95.770 & 0.215 & 2.244 & 0.64 & 64.7 \\
\hline 98.870 & 0.234 & 2.365 & 0.66 & 64.8 \\
\hline 102.100 & 0.342 & 3.344 & 0.68 & 64.7 \\
\hline 105.200 & 0.229 & 2.173 & 0.71 & 64.8 \\
\hline 108.000 & 0.235 & 2.178 & 0.73 & 64.8 \\
\hline 111.300 & 0.183 & 1.640 & 0.75 & 64.8 \\
\hline 113.800 & 0.063 & 0.558 & 0.77 & 64.8 \\
\hline 116.800 & 0.392 & 3.352 & 0.79 & 64.8 \\
\hline 119.500 & 0.422 & 3.530 & 0.81 & 64.8 \\
\hline 122.900 & 0.404 & 3.285 & 0.83 & 64.8 \\
\hline 125.800 & 0.258 & 2.054 & 0.85 & 64.8 \\
\hline 129.200 & 0.219 & 1.693 & 0.87 & 64.8 \\
\hline 131.500 & 0.255 & 1.936 & 0.89 & 64.8 \\
\hline 135.000 & 0.334 & 2.471 & 0.91 & 64.8 \\
\hline 137.800 & 0.269 & 1.950 & 0.93 & 64.8 \\
\hline 141.200 & 0.297 & 2.100 & 0.95 & 64.8 \\
\hline 144.100 & 0.296 & 2.052 & 0.97 & 64.8 \\
\hline 147.000 & 0.496 & 3.372 & 0.99 & 64.8 \\
\hline 149.800 & 0.365 & 2.437 & 1.01 & 64.8 \\
\hline 153.300 & 0.434 & 2.830 & 1.03 & 64.8 \\
\hline 156.100 & 0.288 & 1.843 & 1.05 & 64.8 \\
\hline 159.500 & 0.271 & 1.697 & 1.07 & 64.8 \\
\hline 161.800 & 0.352 & 2.172 & 1.09 & 64.8 \\
\hline 165.300 & 0.370 & 2.238 & 1.11 & 64.8 \\
\hline 168.100 & 0.308 & 1.832 & 1.13 & 64.8 \\
\hline 171.600 & 0.327 & 1.908 & 1.15 & 64.8 \\
\hline 173.800 & 0.448 & 2.579 & 1.17 & 64.8 \\
\hline 177.300 & 0.463 & 2.612 & 1.19 & 64.8 \\
\hline 180.700 & 0.358 & 1.979 & 1.21 & 64.8 \\
\hline 183.600 & 0.198 & 1.078 & 1.23 & 64.8 \\
\hline 187.000 & 0.397 & 2.122 & 1.25 & 64.8 \\
\hline 189.300 & 0.390 & 2.062 & 1.27 & 64.8 \\
\hline 192.700 & 0.285 & 1.478 & 1.29 & 64.8 \\
\hline 195.600 & 0.327 & 1.672 & 1.31 & 64.8 \\
\hline
\end{tabular}


RPP-5798, Rev. 0

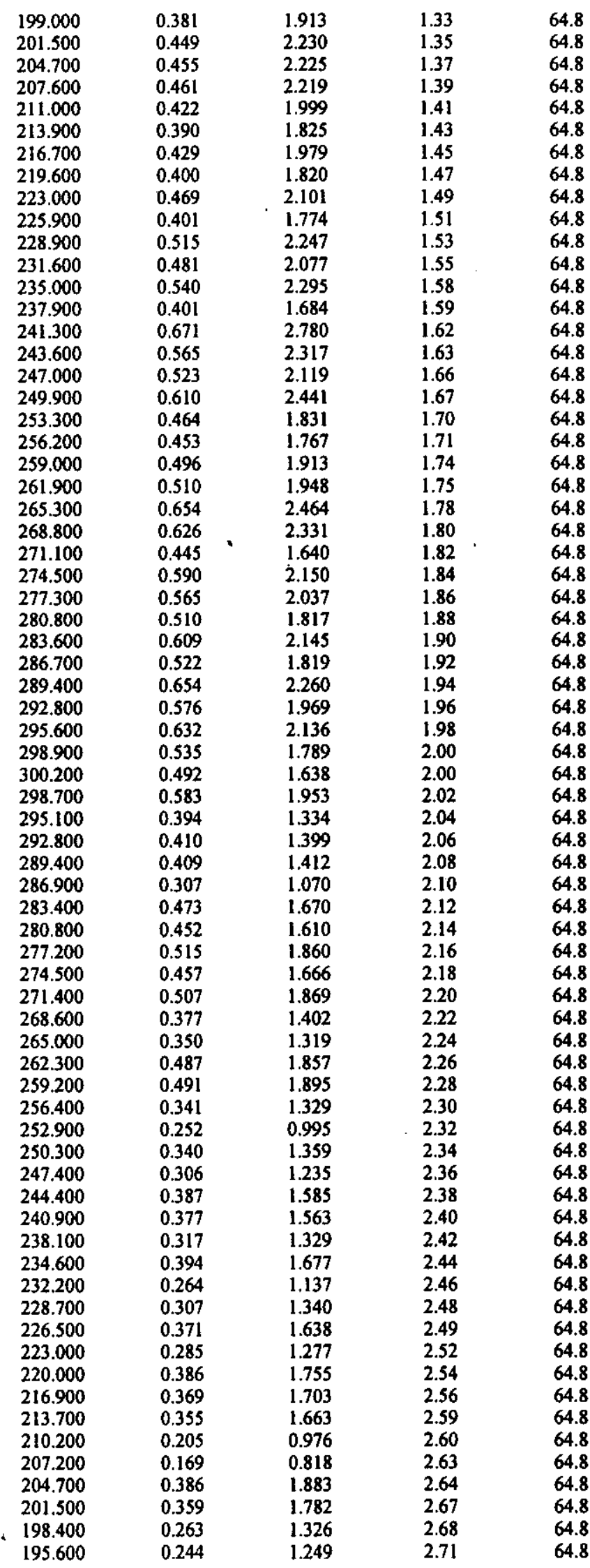


RPP-5798, Rev. 0

\begin{tabular}{|c|c|c|c|c|}
\hline 191.600 & 0.212 & 1.106 & 2.73 & 64.8 \\
\hline 189.300 & 0.280 & 1.480 & 2.75 & 64.8 \\
\hline 186.000 & 0.345 & 1.854 & 2.77 & 64.8 \\
\hline 183.600 & 0.215 & 1.170 & 2.79 & 64.8 \\
\hline 180.100 & 0.240 & 1.334 & 2.80 & 64.8 \\
\hline 177.300 & 0.258 & 1.458 & 2.82 & 64.8 \\
\hline 174.400 & 0.215 & 1.234 & 2.85 & 64.8 \\
\hline 171.400 & 0.167 & 0.975 & 2.87 & 64.8 \\
\hline 168.100 & 0.232 & 1.380 & 2.89 & 64.8 \\
\hline 165.300 & 0.204 & 1.234 & 2.90 & 64.8 \\
\hline 162.600 & $0.35 !$ & 2.159 & 2.92 & 64.8 \\
\hline 159.400 & 0.157 & 0.982 & 2.95 & 64.8 \\
\hline 156.300 & 0.140 & 0.898 & 2.97 & 64.8 \\
\hline 152.500 & 0.271 & 1.778 & 2.99 & 64.8 \\
\hline 150.400 & 0.190 & 1.265 & 3.01 & 64.8 \\
\hline 147.200 & 0.172 & 1.166 & 3.03 & 64.8 \\
\hline 144.500 & 0.296 & 2.047 & 3.05 & 64.8 \\
\hline 141.100 & 0.185 & 1.313 & 3.07 & 64.8 \\
\hline 138.000 & 0.198 & 1.438 & 3.09 & 64.8 \\
\hline 135.000 & 0.232 & 1.719 & 3.11 & 64.8 \\
\hline 132.700 & 0.206 & 1.554 & 3.12 & 64.8 \\
\hline 128.900 & 0.219 & 1.701 & 3.15 & 64.8 \\
\hline 126.000 & 0.199 & 1.579 & 3.17 & 64.8 \\
\hline 122.400 & 0.185 & 1.508 & 3.19 & 64.8 \\
\hline 119.500 & 0.224 & 1.877 & 3.21 & 64.8 \\
\hline 116.700 & 0.203 & 1.739 & 3.23 & 64.8 \\
\hline 113.400 & 0.202 & 1.778 & 3.25 & 64.8 \\
\hline 110.400 & 0.245 & 2.221 & 3.27 & 64.8 \\
\hline 107.500 & 0.263 & 2.447 & 3.29 & 64.8 \\
\hline 106.000 & 0.305 & 2.875 & 3.30 & 64.8 \\
\hline 102.000 & 0.222 & 2.179 & 3.33 & 64.8 \\
\hline 98.970 & 0.192 & 1.944 & 3.35 & 64.8 \\
\hline 95.560 & 0.189 & 1.973 & 3.37 & 64.8 \\
\hline 93.120 & 0.191 & 2.051 & 3.39 & 64.8 \\
\hline 89.720 & 0.139 & 1.543 & 3.41 & 64.8 \\
\hline 86.880 & 0.131 & 1.503 & 3.43 & 64.8 \\
\hline 83.410 & 0.155 & 1.856 & 3.45 & 64.8 \\
\hline 80.360 & 0.289 & 3.597 & 3.47 & 64.8 \\
\hline 77.750 & 0.180 & 2.319 & 3.49 & 64.8 \\
\hline 74.800 & 0.163 & 2.183 & 3.51 & 64.8 \\
\hline 71.310 & 0.156 & 2.192 & 3.53 & 64.8 \\
\hline 68.430 & 0.212 & 3.095 & 3.55 & 64.8 \\
\hline 65.060 & 0.171 & 2.633 & 3.58 & 64.8 \\
\hline 62.710 & 0.181 & 2.888 & 3.59 & 64.8 \\
\hline 59.190 & 0.153 & 2.586 & 3.62 & 64.8 \\
\hline 56.350 & 0.162 & 2.866 & 3.63 & 64.8 \\
\hline 52.900 & 0.158 & 2.982 & 3.66 & 64.8 \\
\hline 50.910 & 0.114 & 2.230 & 3.67 & 64.8 \\
\hline 47.140 & 0.204 & 4.336 & 3.70 & 64.8 \\
\hline 44.200 & 0.124 & 2.798 & 3.71 & 64.8 \\
\hline 40.730 & 0.135 & 3.318 & 3.74 & 64.8 \\
\hline 38.120 & 0.175 & 4.585 & 3.75 & 64.8 \\
\hline 34.960 & 0.172 & 4.925 & 3.78 & 64.8 \\
\hline 31.570 & 0.176 & 5.559 & 3.80 & 64.8 \\
\hline 28.730 & 0.207 & 7.212 & 3.82 & 64.8 \\
\hline 25.240 & 0.133 & 5.257 & 3.84 & 64.8 \\
\hline 22.840 & 0.150 & 6.563 & 3.86 & 64.8 \\
\hline 19.460 & 0.117 & 5.987 & 3.88 & 64.8 \\
\hline 16.620 & 0.146 & 8.772 & 3.90 & 64.8 \\
\hline 13.150 & 0.106 & 8.090 & 3.92 & 64,8 \\
\hline 10.410 & 0.153 & 14.670 & 3.94 & 64,8 \\
\hline 7.415 & 0.116 & 15.690 & 3.96 & 64.8 \\
\hline 4.537 & 0.086 & 19.040 & 3.98 & 64.8 \\
\hline 1.144 & 0.074 & 64.980 & 4.00 & 64,8 \\
\hline
\end{tabular}


RPP-5798, Rev. 0

VISCOȘITY ANALYSIS OF

DECANTED SUPERNATANT LIQUID

AT AMBIENT TEMPERATURE 
RPP-5798, Rev. 0

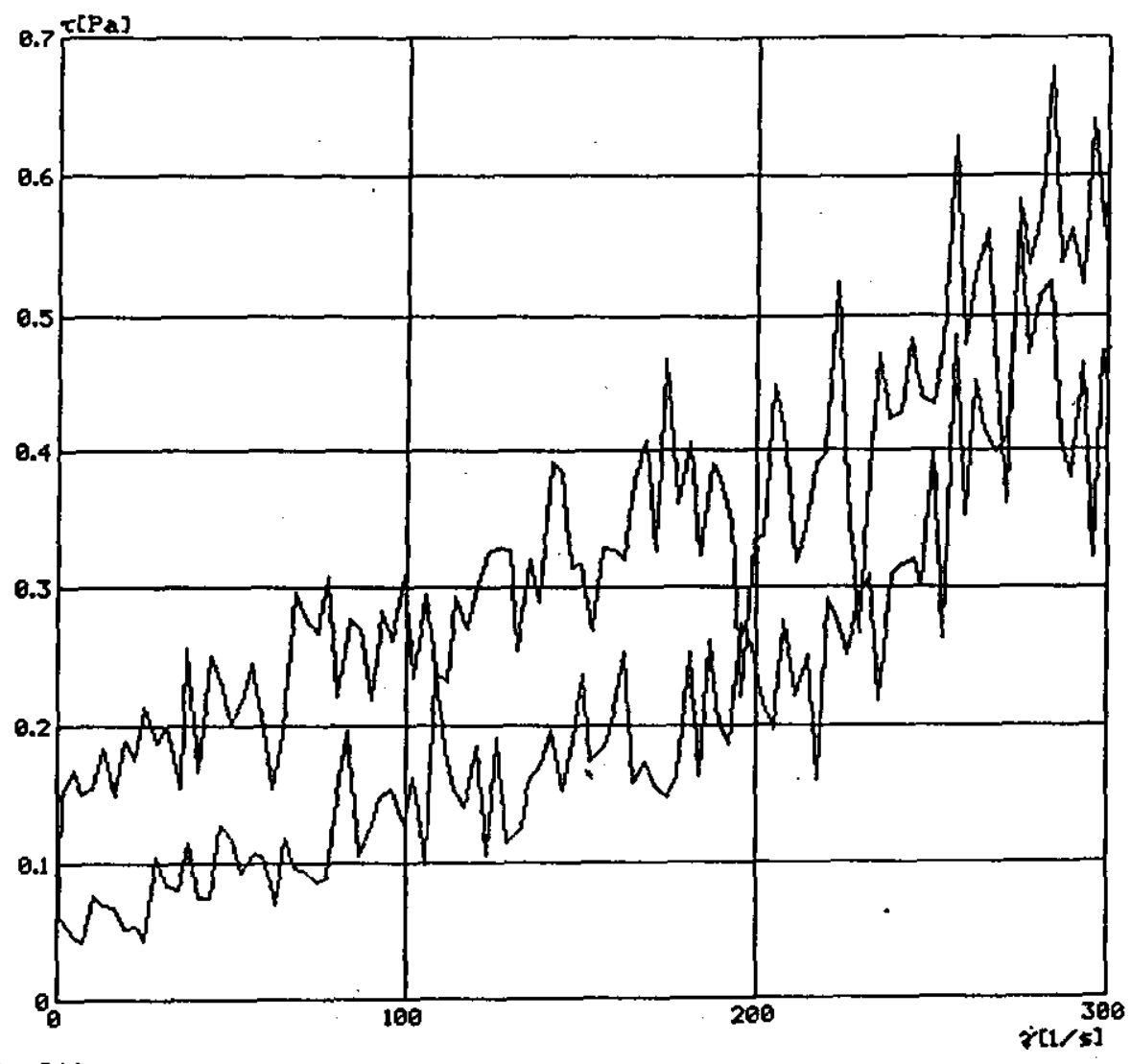

H A A K

Operator:

jwe

Substance:

0104 to

supernate only

Test No.:

01

Test of:

09-13-1999

System:

MS/MV1

Temporature:

$23.0^{\circ} \mathrm{C}$

D- C4TEBRT.ROT

MAQKF RAK SA Y

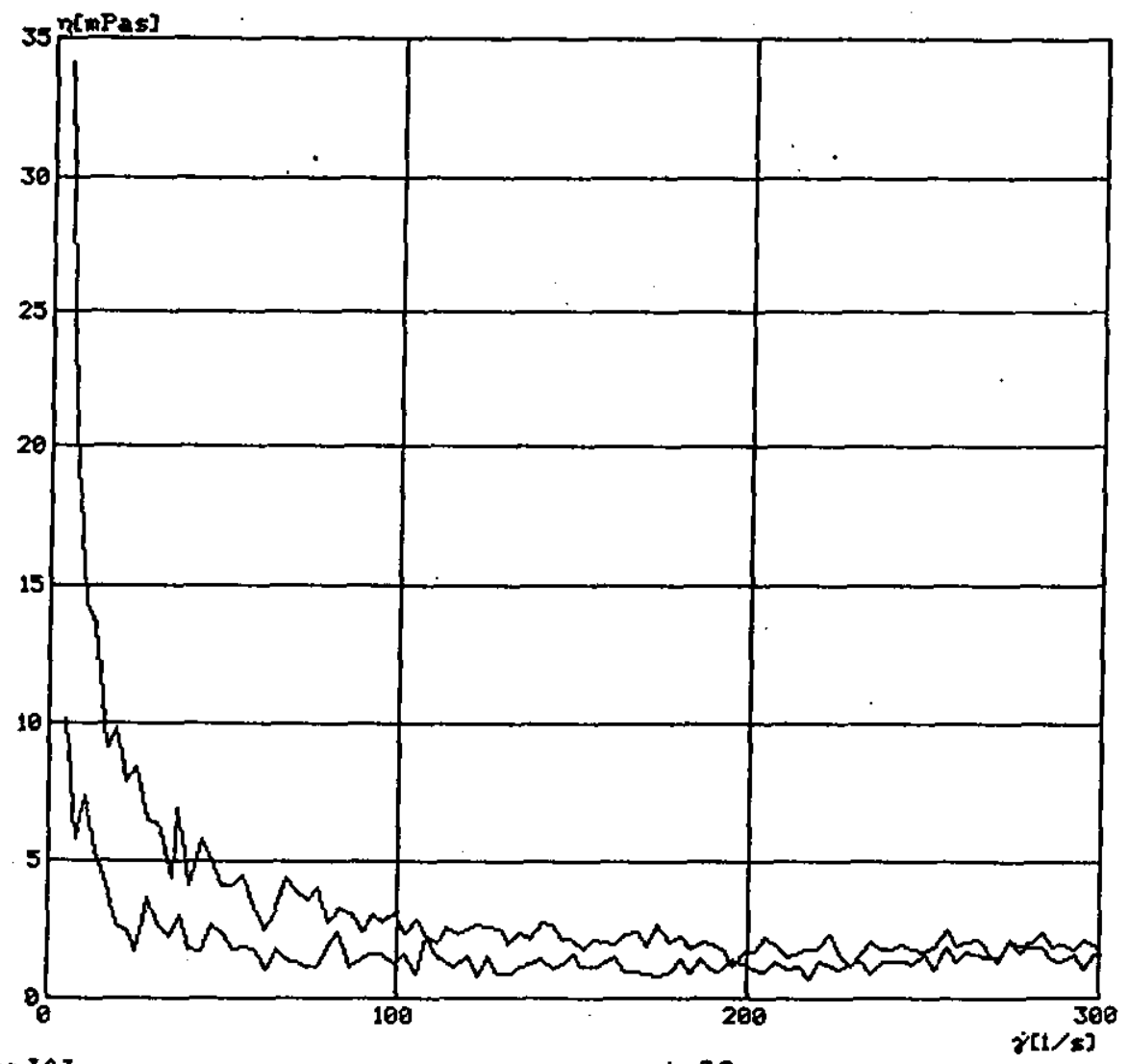

Hคค $\boldsymbol{A} \mathbf{E}$

Operator:

jwo

Substance?

0104 to

supernate only

Test No.:

(1)

Test of:

09-13-1999

System:

M5/MV1

Tempersture:

$23.0^{\circ} \mathrm{C}$ 
RPP-5798, Rev. 0

\begin{tabular}{|c|c|c|c|c|}
\hline$\tau[\mathbf{P a}]$ & $\gamma\left[\mathbf{s}^{-1}\right]$ & $\eta[$ mPas] & $t[\mathrm{~min}]$ & Temp $\mid{ }^{\circ} \mathrm{C}$ \\
\hline 0.000 & 0.028 & 0.000 & 0.00 & 28.9 \\
\hline 1.468 & 0.151 & 102.900 & 0.02 & 28.9 \\
\hline 4.918 & 0.168 & 34.170 & 0.04 & 28.9 \\
\hline 7.262 & 0.151 & 20.740 & 0.06 & 28.9 \\
\hline 10.640 & 0.154 & 14.430 & 0.08 & 28.9 \\
\hline 13.510 & 0.184 & 13.620 & 0.10 & 28.9 \\
\hline 16.490 & 0.150 & 9.065 & 0.11 & 28.9 \\
\hline 19.330 & 0.190 & 9.830 & 0.14 & 28.9 \\
\hline 22.210 & 0.174 & 7.845 & 0.15 & 28.9 \\
\hline 25.410 & 0.214 & 8.426 & 0.18 & 28.9 \\
\hline 28.860 & 0.187 & 6.467 & 0.20 & 28.9 \\
\hline 31.450 & 0.200 & $6.37 i$ & 0.22 & 28.9 \\
\hline 35.490 & 0.154 & 4.345 & 0.24 & 28.9 \\
\hline 37.250 & 0.256 & 6.877 & 0.26 & 28.9 \\
\hline 40.410 & 0.166 & 4.111 & 0.28 & 28.9 \\
\hline 43.820 & 0.251 & 5.726 & 0.30 & 28.9 \\
\hline 47.430 & 0.228 & 4.803 & 0.32 & 28.9 \\
\hline 49.870 & 0.202 & 4.044 & 0.34 & 28.9 \\
\hline 53.070 & 0.217 & 4.097 & 0.36 & 28.9 \\
\hline 55.980 & 0.246 & 4.390 & 0.38 & 28.9 \\
\hline 59.490 & 0.198 & 3.321 & 0.40 & 28.9 \\
\hline 61.970 & 0.155 & 2.494 & 0.41 & 28.9 \\
\hline 65.230 & 0.196 & 3.000 & 0.44 & 28.9 \\
\hline 68.200 & 0.297 & 4.351 & 0.46 & 28.9 \\
\hline 71.600 & 0.279 & 3.901 & 0.48 & 28.9 \\
\hline 74.490 & 0.268 & 3.591 & 0.50 & 28.9 \\
\hline 77.490 & 0.307 & 3.964 & 0.52 & 28.9 \\
\hline 80.290 & 0.220 & 2.743 & 0.54 & 29.0 \\
\hline 83.740 & 0.278 & 3.318 & 0.56 & 28.9 \\
\hline 86.580 & 0.271 & 3.128 & 0.58 & 28.9 \\
\hline 89.910 & 0.219 & 2.434 & 0.60 & 29.0 \\
\hline 92.980 & 0.282 & 3.034 & 0.63 & 28.9 \\
\hline 95.880 & 0.261 & 2.721 & 0.64 & 28.9 \\
\hline 99.290 & 0.310 & 3,117 & 0.67 & 28.9 \\
\hline 102.200 & 0.235 & 2.299 & 0.68 & 28.9 \\
\hline 105.200 & 0.295 & 2.808 & 0.71 & 28.9 \\
\hline 108.000 & 0.238 & 2.206 & 0.73 & 28.9 \\
\hline 111.500 & 0.231 & 2.068 & 0.75 & 29.0 \\
\hline 113.600 & 0.294 & 2.583 & 0.77 & 29.0 \\
\hline 117.200 & 0.270 & 2.306 & 0.79 & 29.0 \\
\hline 119.500 & 0.295 & 2.467 & 0.81 & 29.0 \\
\hline 122.900 & 0.326 & 2.648 & 0.83 & 29.0 \\
\hline 125.800 & 0.329 & 2.614 & 0.85 & 29.0 \\
\hline 129.200 & 0.327 & 2.527 & 0.87 & 29.0 \\
\hline 131.700 & 0.254 & 1.930 & 0.89 & 29.0 \\
\hline 135.000 & 0.319 & 2.364 & 0.91 & 29.0 \\
\hline 137.800 & 0.290 & 2.105 & 0.93 & 29.0 \\
\hline 141.200 & 0.392 & 2.777 & 0.95 & 29.0 \\
\hline 144.100 & 0.386 & 2.676 & 0.97 & 29.0 \\
\hline 147.000 & 0.314 & 2.138 & 0.99 & 29.0 \\
\hline 149.800 & 0.316 & 2.107 & 1.01 & 28.9 \\
\hline 153.300 & 0.268 & 1.752 & 1.03 & 29.0 \\
\hline 156.100 & 0.328 & 2.101 & 1.05 & 29.0 \\
\hline 159.500 & 0.327 & 2.050 & 1.07 & 28.9 \\
\hline 161.800 & 0.320 & 1.974 & 1.09 & 29.0 \\
\hline 165.300 & 0.380 & 2.299 & 1.11 & 29.0 \\
\hline 168.100 & 0.407 & 2.423 & 1.13 & 29.0 \\
\hline 171.600 & 0.327 & 1.903 & 1.15 & 28.9 \\
\hline 173.800 & 0.467 & 2.688 & 1.17 & 29.0 \\
\hline 177.300 & 0.362 & 2.045 & 1.19 & 28.9 \\
\hline 180.700 & 0.406 & 2.249 & 1.21 & 28.9 \\
\hline 183.600 & 0.323 & 1.761 & 1.23 & 28.9 \\
\hline 187.000 & 0.390 & 2.087 & 1.25 & 28.9 \\
\hline 189.300 & 0.379 & 2.002 & 1.27 & 28.9 \\
\hline 192.700 & 0.348 & 1.807 & 1.29 & 29.0 \\
\hline 195.600 & 0.221 & 1.129 & 1.31 & 28.9 \\
\hline
\end{tabular}


RPP-5798, Rev. 0

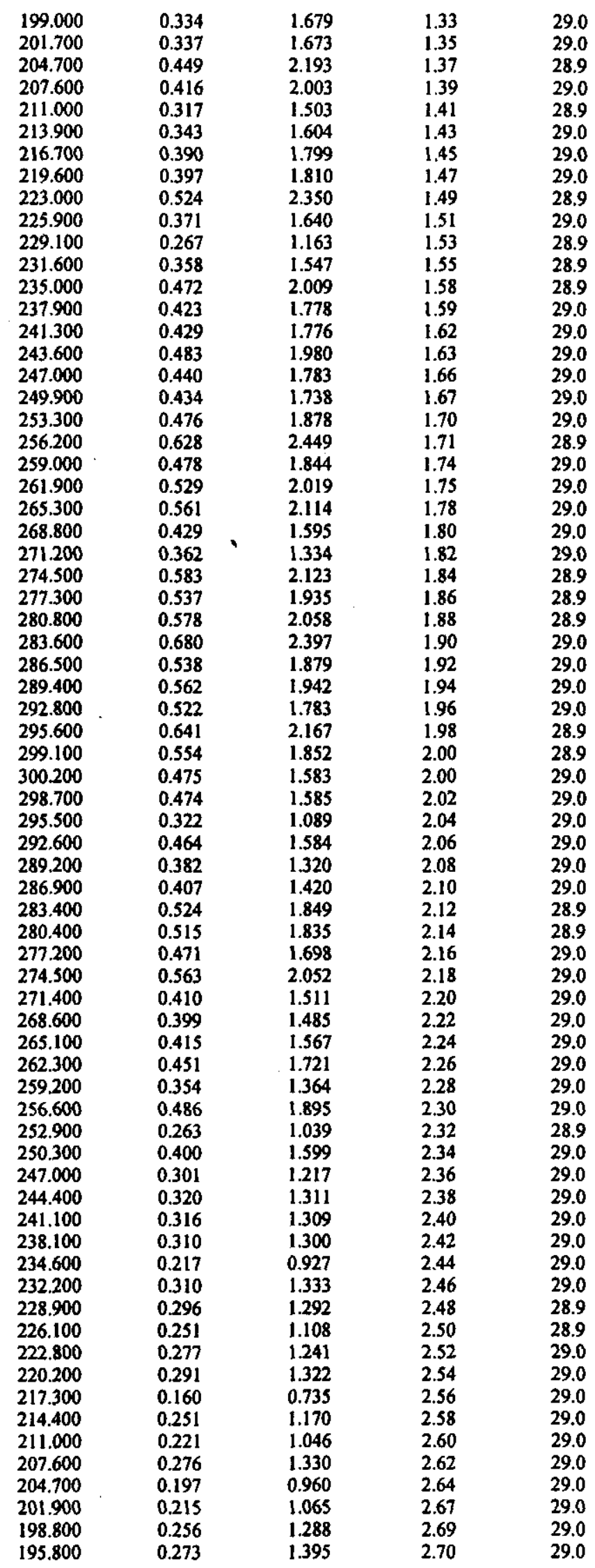


RPP-5798, Rev. 0

\begin{tabular}{|c|c|c|c|c|}
\hline 192.100 & 0.185 & 0.962 & 2.73 & 29.0 \\
\hline 189.500 & 0.200 & 1.055 & 2.75 & 29.0 \\
\hline 186.600 & 0.261 & 1.400 & 2.77 & 29.0 \\
\hline 183.600 & 0.162 & 0.884 & 2.78 & 29.0 \\
\hline 180.700 & 0.252 & 1.397 & 2.80 & 29.0 \\
\hline 177.300 & 0.163 & 0.922 & 2.83 & 29.0 \\
\hline 174.600 & 0.148 & 0.846 & 2.85 & 29.0 \\
\hline 171.200 & 0.155 & 0.907 & 2.87 & 29.0 \\
\hline 168.500 & 0.173 & 1.028 & 2.89 & 29.0 \\
\hline 165.300 & 0.157 & 0.952 & 2.91 & 29.0 \\
\hline 161.800 & 0.252 & 1.556 & 2.93 & 29.0 \\
\hline 159.000 & 0.202 & 1.269 & 2.95 & 29.0 \\
\hline 156.700 & 0.183 & 1.169 & 2.97 & 29.0 \\
\hline 152.500 & 0.173 & 1.133 & 2.99 & 28.9 \\
\hline 150.200 & 0.237 & 1.579 & 3.00 & 29.0 \\
\hline 147.300 & 0.189 & 1.280 & 3.03 & 29.0 \\
\hline 144.500 & 0.152 & 1.049 & 3.05 & 28.9 \\
\hline 141.100 & 0.196 & 1.391 & 3.07 & 29.0 \\
\hline 138.400 & 0.172 & 1.242 & 3.09 & 28.9 \\
\hline 135.000 & 0.160 & 1.186 & 3.11 & 29.0 \\
\hline 132.700 & 0.125 & 0.939 & 3.13 & 29.0 \\
\hline 128.700 & 0.114 & 0.883 & 3.15 & 29.0 \\
\hline 125.600 & 0.191 & 1.516 & 3.17 & 29.0 \\
\hline 122.800 & 0.105 & 0.856 & 3.19 & 28.9 \\
\hline 119.900 & 0.186 & 1.550 & 3.21 & 28.9 \\
\hline 116.800 & 0.139 & 1.192 & 3.23 & 28.9 \\
\hline 113.600 & 0.153 & 1.343 & 3.25 & 29.0 \\
\hline 110.900 & 0.182 & 1.639 & 3.27 & 29.0 \\
\hline 108.100 & 0.240 & 2.217 & 3.29 & 29.0 \\
\hline 105.700 & 0.098 & 0.923 & 3.31 & 29.0 \\
\hline 101.900 & 0.162 & 1.591 & 3.33 & 29.0 \\
\hline 98.950 & 0.127 & 1.287 & 3.35 & 29.0 \\
\hline 95.500 & 0.153 & 1.603 & 3.37 & 29.0 \\
\hline 92.430 & 0.146 & 1.580 & 3.39 & 29.0 \\
\hline 89.890 & 0.127 & 1.417 & 3.41 & 29.0 \\
\hline 86.830 & 0.105 & 1.208 & 3.43 & 28.9 \\
\hline 82.940 & 0.196 & 2.365 & 3.45 & 28.9 \\
\hline 80.670 & 0.149 & 1.850 & 3.47 & 29.0 \\
\hline 77.580 & 0.088 & 1.137 & 3.49 & 29.0 \\
\hline 74.740 & 0.084 & 1.128 & 3.51 & 28.9 \\
\hline 71.290 & 0.093 & 1.307 & 3.53 & 28.9 \\
\hline 68.410 & 0.096 & 1.406 & 3.55 & 29.0 \\
\hline 65.340 & 0.118 & 1,806 & 3.58 & 29.0 \\
\hline 62.580 & 0.069 & 1.096 & 3.59 & 29.0 \\
\hline 59.170 & 0.105 & 1.766 & 3.62 & 29. \\
\hline 56.330 & 0.106 & 1.881 & 3.63 & 29.0 \\
\hline 52.840 & 0.092 & 1.745 & 3.66 & 28.9 \\
\hline 50.420 & 0.117 & 2.315 & 3.67 & 28.9 \\
\hline 47.080 & 0.127 & 2.692 & 3.70 & 29.0 \\
\hline 44.150 & 0.074 & 1.672 & 3.71 & 29.0 \\
\hline 40.720 & 0.074 & 1.822 & 3.74 & 28.9 \\
\hline 37.990 & 0.115 & 3.018 & 3.75 & 28.9 \\
\hline 34.920 & 0.079 & 2.263 & 3.78 & 29.0 \\
\hline 31.550 & 0.084 & 2.662 & 3.80 & 28.9 \\
\hline 28.650 & 0.105 & 3.672 & 3.82 & 29.0 \\
\hline 25.160 & 0.042 & 1.687 & 3.84 & 29.0 \\
\hline 22.740 & 0.054 & 2.382 & 3.86 & 29.0 \\
\hline 19.440 & 0.052 & 2.681 & 3.88 & 28.9 \\
\hline 16.600 & 0.068 & 4.102 & 3.90 & 28. \\
\hline 13.130 & 0.070 & 5.329 & 3.92 & 28.9 \\
\hline 10.370 & 0.076 & 7.337 & 3.94 & 28.9 \\
\hline 7.377 & 0.042 & 5.735 & 3.96 & 28.9 \\
\hline 4.537 & 0.046 & 10.200 & 3.98 & 28.9 \\
\hline 1.087 & 0.058 & 53.680 & 4.00 & 28.9 \\
\hline
\end{tabular}


RPP-5798, Rev. 0

\section{VISCOṠITY ANALYSIS OF}

DECANTED SUPERNATANT LIQUID AT $45^{\circ} \mathrm{C}$ 
RPP-5798, Rev. 0

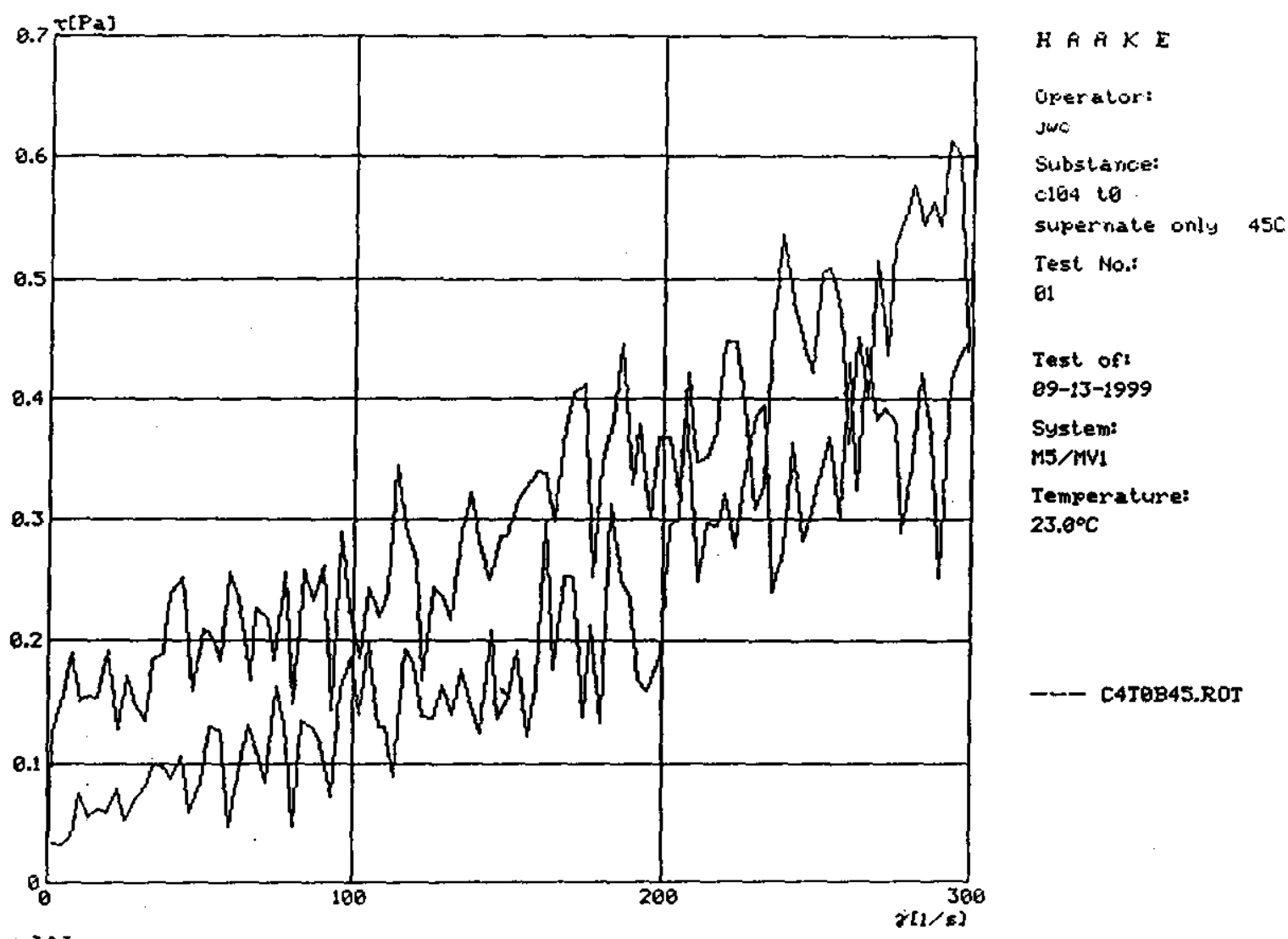

WAQXF RnT Bax

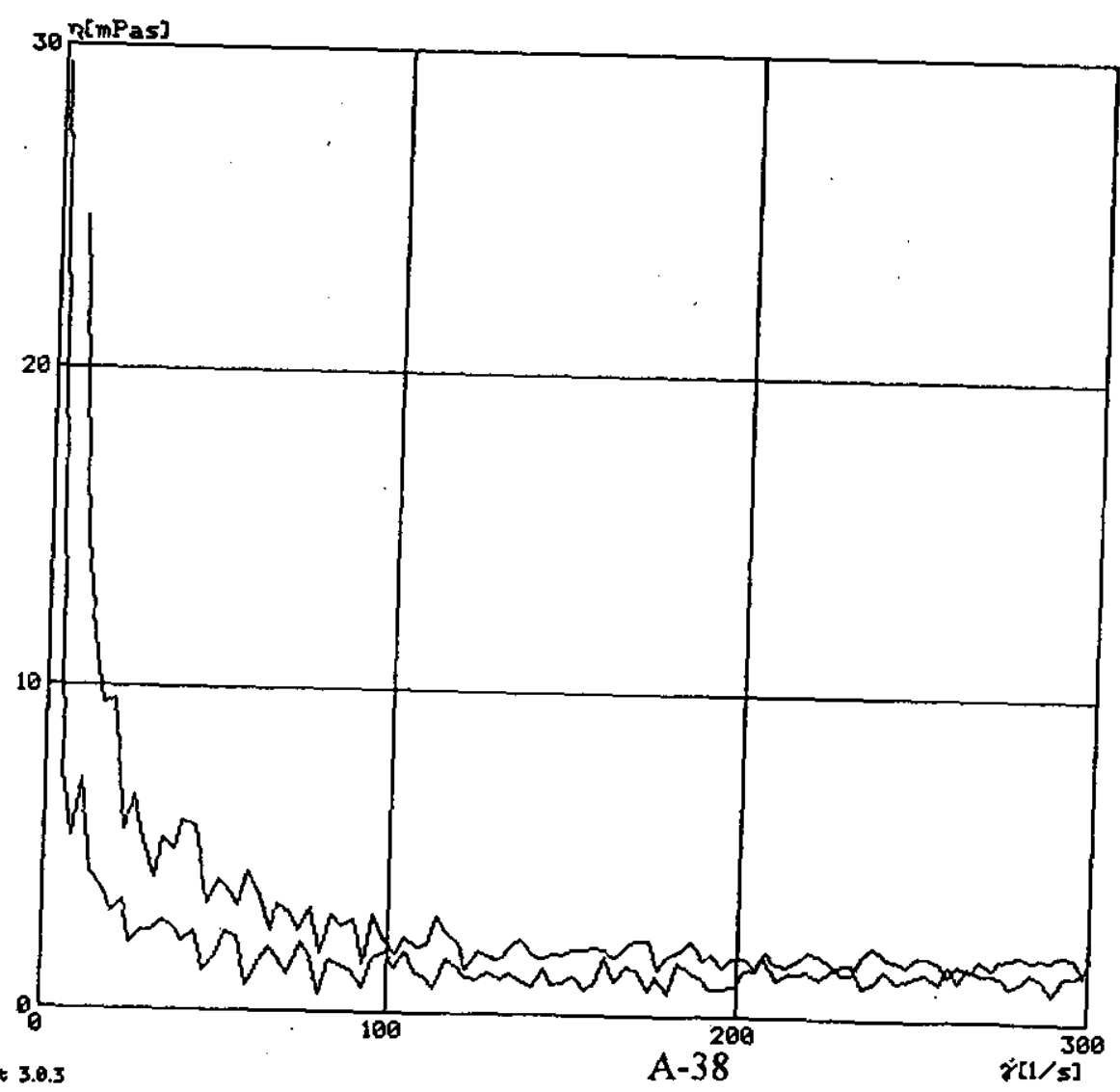

H ค ค $K \mathbf{E}$

Operator:

two

Substance:

c104 to

supernate only $45 \mathrm{C}$

Test No.:

ot

Test of:

09-13-1999

System:

MS/MV1

Temperature:

$23.0^{\circ} \mathrm{C}$ 
RPP-5798, Rev. 0

\begin{tabular}{|c|c|c|c|c|}
\hline$\tau[\mathrm{Pa}]$ & $\gamma\left[s^{-1}\right]$ & $\eta[\mathrm{mPas}]$ & t|min] & Temp $\mid{ }^{\circ} \mathrm{C}$ \\
\hline 0.000 & 0.009 & 0.000 & 0.00 & 45.4 \\
\hline 1.468 & 0.125 & 85.220 & 0.02 & 45.4 \\
\hline 4.308 & 0.149 & 34.580 & 0.04 & 45.4 \\
\hline 7.701 & 0.190 & 24.650 & 0.06 & 45.4 \\
\hline 10.240 & 0.150 & 14,610 & 0.08 & 45.4 \\
\hline 13.480 & 0.155 & 11.520 & 0.10 & 45.4 \\
\hline 16.350 & 0.155 & 9.462 & 0.12 & 45.4 \\
\hline 19.790 & 0.190 & 9.617 & 0.14 & 45.4 \\
\hline 22.820 & 0.128 & 5.621 & 0.15 & 45.4 \\
\hline 25.520 & 0.171 & 6.698 & 0.18 & 45.4 \\
\hline 28.380 & 0.148 & 5.206 & 0.20 & 45.4 \\
\hline 32.420 & 0.134 & 4.130 & 0.22 & 45.4 \\
\hline 34.080 & 0.184 & 5.411 & 0.23 & 45.4 \\
\hline 37.670 & 0.189 & 5.007 & 0.26 & 45.4 \\
\hline 40.090 & 0.239 & 5.951 & 0.28 & 45.4 \\
\hline 43.920 & 0.253 & 5.766 & 0.30 & 45.3 \\
\hline 47.350 & 0.160 & 3.372 & 0.32 & 45.3 \\
\hline 50.720 & 0.209 & 4.126 & 0.34 & 45.3 \\
\hline 53.220 & 0.205 & 3.843 & 0.36 & 45.3 \\
\hline 56.780 & 0.184 & 3.244 & 0.38 & 45.3 \\
\hline 59.210 & 0.257 & 4.333 & 0.40 & 45.3 \\
\hline 62.430 & 0.236 & 3.776 & 0.42 & 45.3 \\
\hline 66.160 & 0.168 & 2.535 & 0.44 & 45.3 \\
\hline 67.840 & 0.225 & 3.322 & 0.46 & 45.3 \\
\hline 71.610 & 0.218 & 3.041 & 0.48 & 45.3 \\
\hline 74.320 & 0.185 & 2.485 & 0.50 & 45.3 \\
\hline 77.520 & 0.256 & 3.297 & 0.52 & 45.3 \\
\hline 80.520 & 0.148 & 1.842 & 0.54 & 45.3 \\
\hline 83.830 & 0.258 & 3.071 & 0.56 & 45.3 \\
\hline 86.480 & 0.233 & 2.698 & 0.58 & 45.3 \\
\hline 89.820 & 0.261 & 2.903 & 0.60 & 45.3 \\
\hline 93.040 & 0.143 & 1.538 & 0.62 & 45.2 \\
\hline 95.380 & 0.291 & 3.046 & 0.65 & 45.3 \\
\hline 98.660 & 0.226 & 2.289 & 0.66 & 45.2 \\
\hline 102.100 & 0.185 & 1.816 & 0.69 & 45.2 \\
\hline 105.000 & 0.244 & 2.326 & 0.70 & 45.2 \\
\hline 108.300 & 0.219 & 2.025 & 0.73 & 45.2 \\
\hline 110.900 & 0.241 & 2.169 & 0.74 & 45.2 \\
\hline 113.600 & 0.345 & 3.040 & 0.77 & 45.2 \\
\hline 116.700 & 0.295 & 2.527 & 0.78 & 45.2 \\
\hline 120.100 & 0.266 & 2.211 & 0.81 & 45.2 \\
\hline 122.400 & 0.167 & 1.367 & 0.82 & 45.2 \\
\hline 125,400 & 0.244 & 1.947 & 0.85 & 45.2 \\
\hline 128.900 & 0.237 & 1.840 & 0.87 & 45.2 \\
\hline 131.700 & 0.217 & 1.646 & 0.89 & 45.2 \\
\hline 135.100 & 0.290 & 2.143 & 0.91 & 45.1 \\
\hline 137.800 & 0.324 & 2.348 & 0.93 & 45.1 \\
\hline 141.200 & 0.274 & 1.940 & 0.95 & 45.1 \\
\hline 144.100 & 0.251 & 1.744 & 0.97 & 45.1 \\
\hline 147.500 & 0.285 & 1.934 & 0.99 & 45.1 \\
\hline 149.800 & 0.286 & 1.908 & 1.01 & 45.1 \\
\hline 153.300 & 0.317 & 2.068 & 1.03 & 45.1 \\
\hline 156.100 & 0.327 & 2.094 & 1.05 & 45.1 \\
\hline 159.500 & 0.340 & 2.132 & 1.07 & 45.1 \\
\hline 162.400 & 0.338 & 2.083 & 1.09 & 45.1 \\
\hline 165.300 & 0.299 & 1.809 & 1.11 & 45.1 \\
\hline 168.100 & 0.376 & 2.234 & 1.13 & 45.1 \\
\hline 171.600 & 0.405 & 2.363 & 1.15 & 45.1 \\
\hline 174.400 & 0.412 & 2.365 & 1.17 & 45.1 \\
\hline 177.500 & 0.252 & 1.419 & 1.19 & 45.1 \\
\hline 180.100 & 0.349 & 1.936 & 1.21 & 45.1 \\
\hline 183.600 & 0.381 & 2.077 & 1.23 & 45.1 \\
\hline 186.400 & 0.446 & 2.392 & 1.25 & 45.1 \\
\hline 189.900 & 0.330 & 1.737 & 1.27 & 45.1 \\
\hline 192.100 & 0.379 & 1.974 & 1.29 & 45.1 \\
\hline 195.600 & 0.300 & 1.534 & 1.31 & 45.1 \\
\hline
\end{tabular}


RPP-5798, Rev. 0

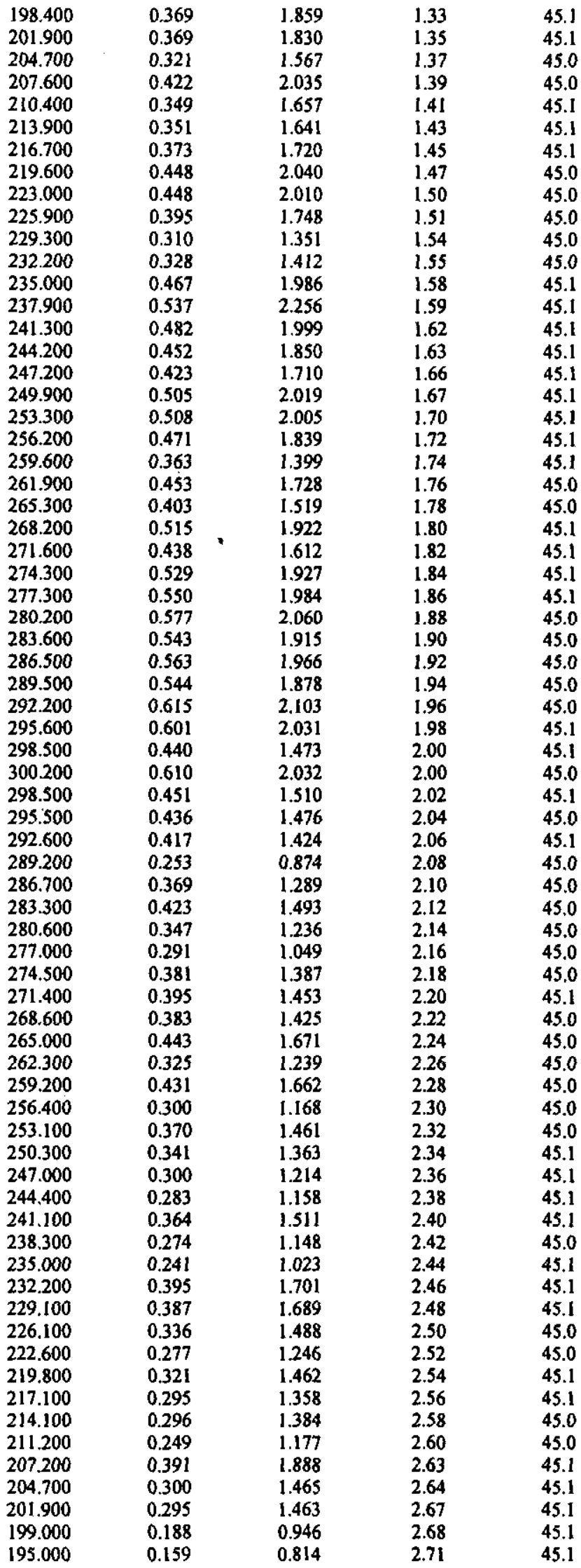


RPP-5798, Rev. 0

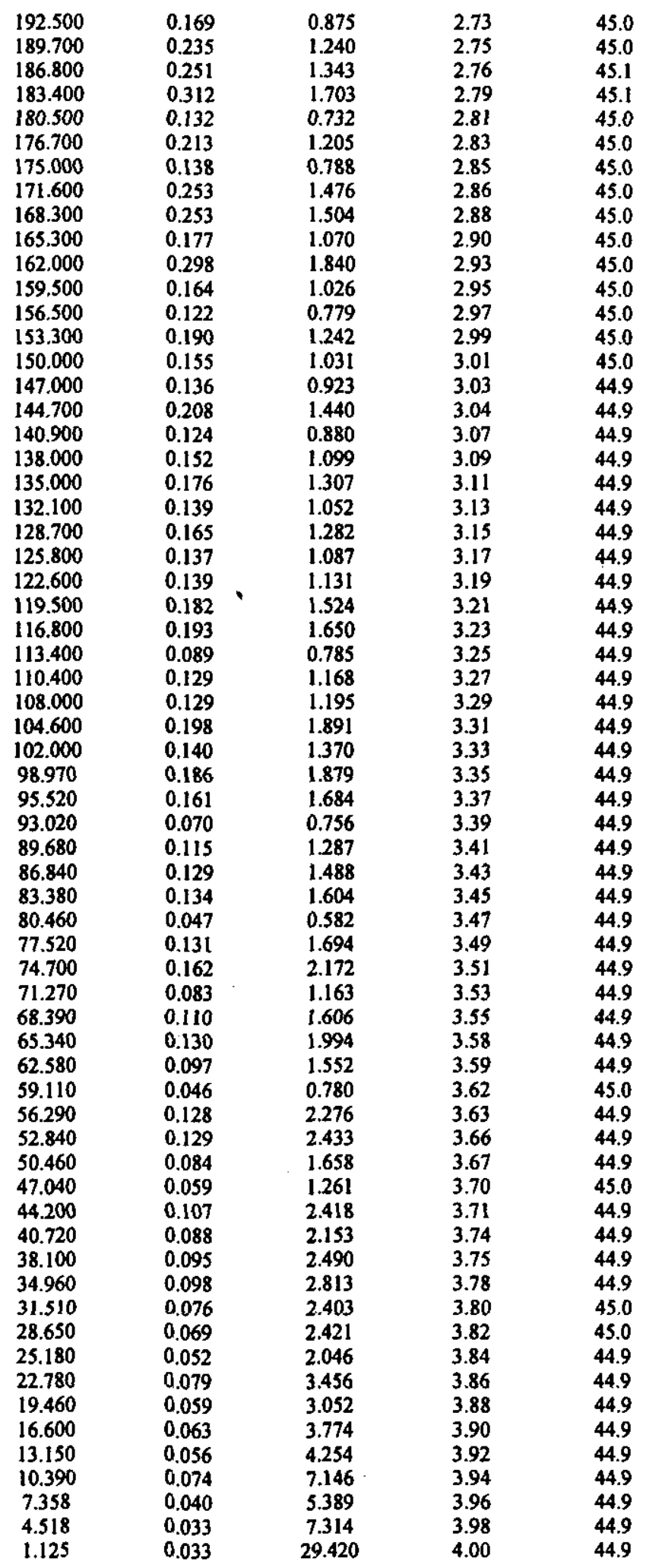


RPP-5798, Rev. 0

\section{VISCOSITY ANALYSIS OF}

\section{DECANTED SUPERNATANT LIQUID AT $65^{\circ} \mathrm{C}$}


RPP-5798, Rev. 0

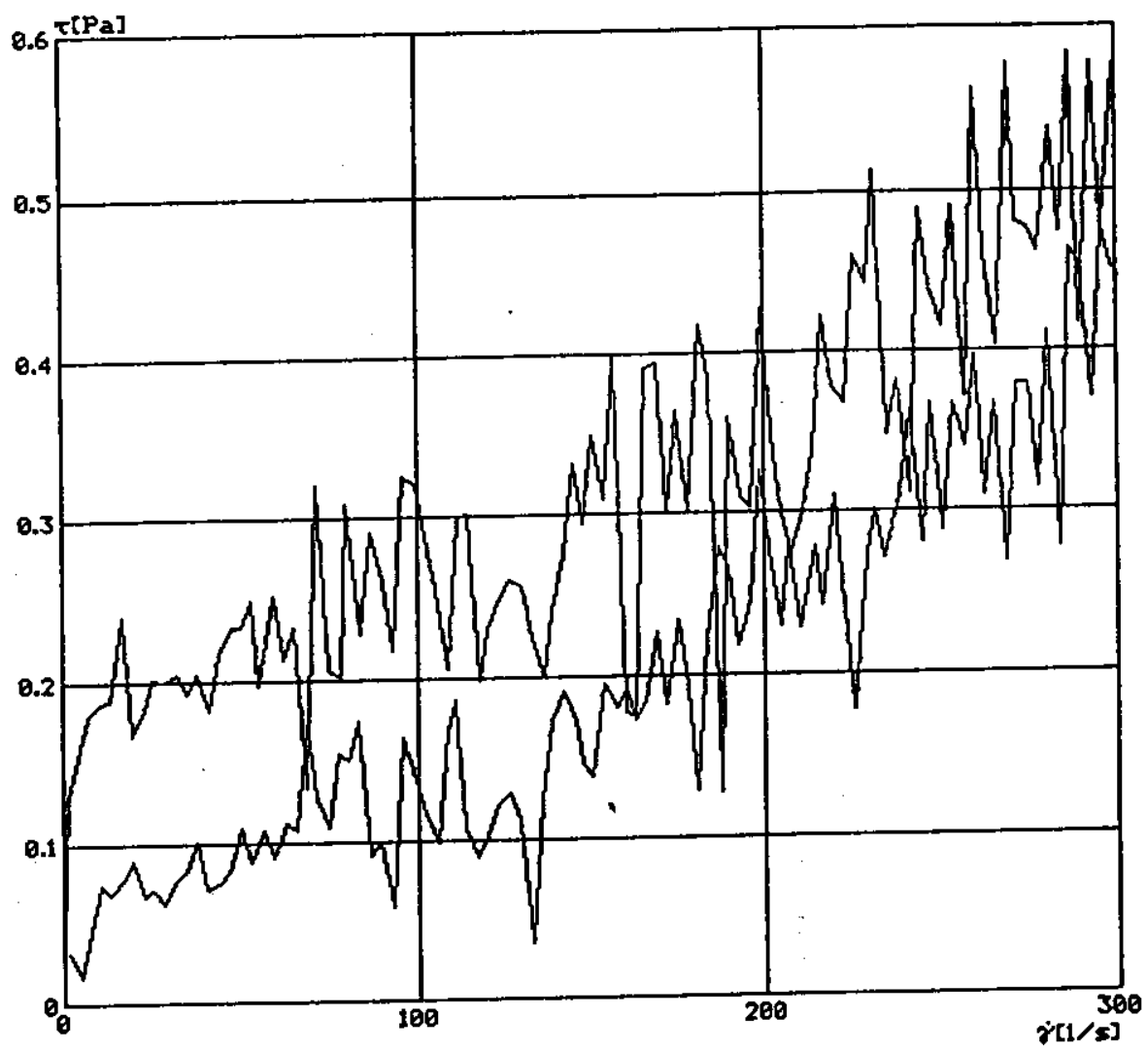

H ค ค X E

Operator:

jwo

Substances

c104 to

supernate only $65 \mathrm{C}$

Test No.:

01

Test of:

09-13-1999

System:

MS/MVI

Temperature:

$23.0^{\circ} \mathrm{C}$

C4TOB65.ROT

HRAKE ROE 3.0 .3

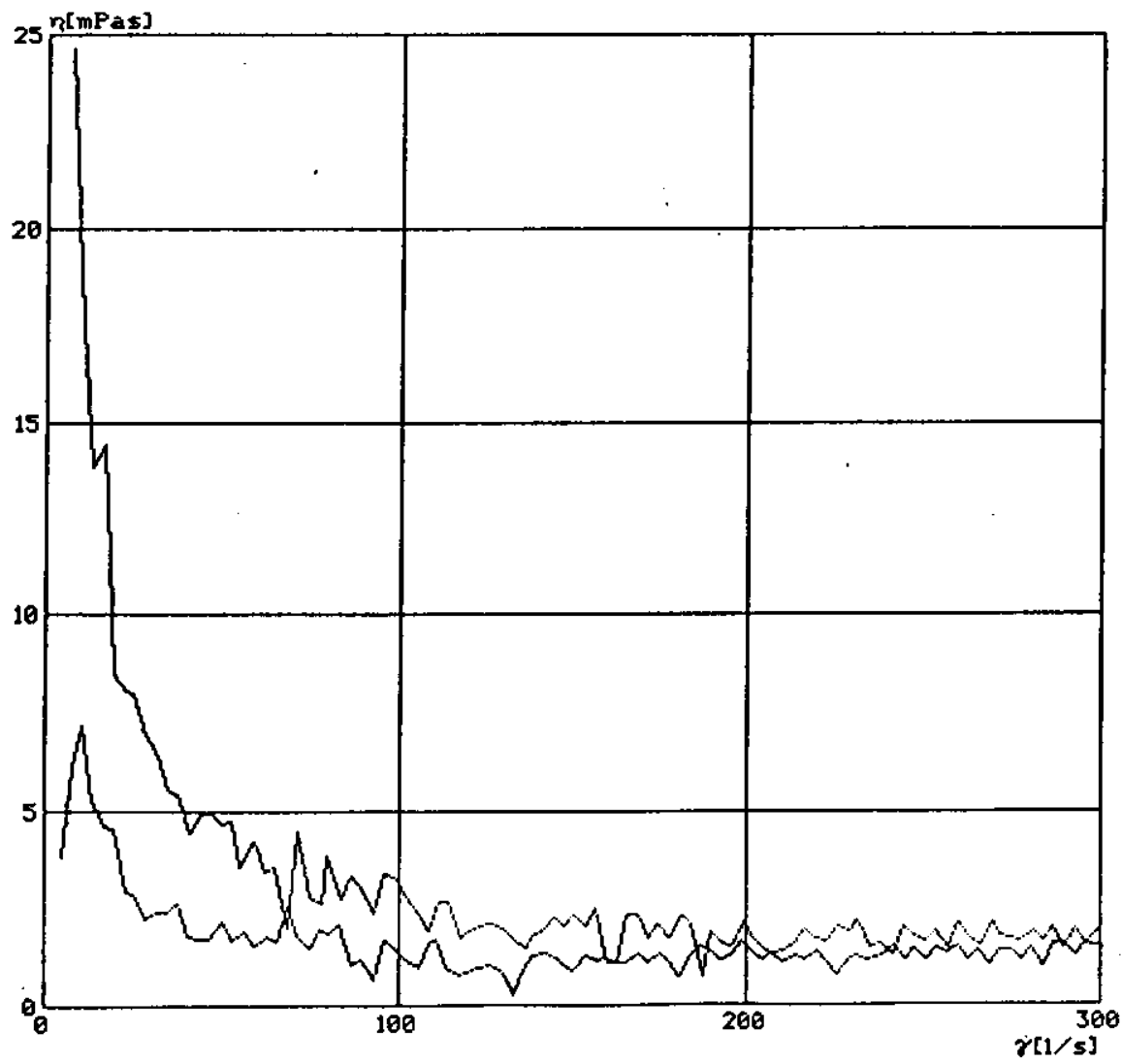

H A $\mathrm{K}$

Operator:

jwe

Substance:

c104 to

supernate only $65 \mathrm{C}$

Test No.:

อ1

Test of:

69-13-1999

System:

M5/MVI

Temperature:

$23.0^{\circ} \mathrm{C}$

C4TOB65.ROT 
RPP-5798, Rev. 0

\begin{tabular}{|c|c|c|c|c|}
\hline$\tau[\mathbf{P a}]$ & $\gamma\left[\mathrm{s}^{-1}\right]$ & $\eta[\mathbf{m P a s}]$ & $\mathrm{t}[\mathrm{min}]$ & Temp $\left.\left.\right|^{\circ} \mathrm{C}\right]$ \\
\hline 0.000 & 0.036 & 0.000 & 0.00 & 65.0 \\
\hline 1.468 & 0.131 & 89.200 & 0.02 & 64.9 \\
\hline 4.899 & 0.160 & 32.550 & 0.04 & 64.9 \\
\hline 7.243 & 0.178 & 24.620 & 0.06 & 64.9 \\
\hline 10.640 & 0.186 & 17.510 & 0.08 & 64.9 \\
\hline 13.530 & 0.188 & 13.900 & 0.10 & 65.0 \\
\hline 16.620 & 0.241 & 14.470 & 0.12 & 64.9 \\
\hline 19.630 & 0.167 & 8.493 & 0.14 & 64.9 \\
\hline 22.660 & 0.183 & 8.076 & 0.16 & 65.0 \\
\hline 25.120 & 0.202 & 8.027 & 0.18 & 65.0 \\
\hline 28.940 & 0.200 & 6.924 & 0.20 & 65.0 \\
\hline 31.790 & 0.205 & 6.436 & 0.22 & 65.0 \\
\hline 34.880 & 0.193 & 5.533 & 0.24 & 65.0 \\
\hline 37.780 & 0.205 & 5.429 & 0.26 & 65.0 \\
\hline 41.500 & 0.183 & 4.398 & 0.28 & 65.0 \\
\hline 43.770 & 0.216 & 4.946 & 0.30 & 65.0 \\
\hline 47.250 & 0.233 & 4.930 & 0.32 & 65.0 \\
\hline 49.960 & 0.233 & 4.654 & 0.34 & 64.9 \\
\hline 53.120 & 0.251 & 4.715 & 0.36 & 65.0 \\
\hline 55.450 & 0.197 & 3.545 & 0.38 & 65.0 \\
\hline 59.410 & 0.253 & 4.255 & 0.40 & 65.0 \\
\hline 62.180 & 0.214 & 3.435 & 0.42 & 65.1 \\
\hline 65.230 & 0.233 & 3.565 & 0.44 & 65.0 \\
\hline 68.320 & 0.133 & 1.944 & 0.46 & 65.0 \\
\hline 71.440 & 0.321 & 4.491 & 0.48 & 65.0 \\
\hline 74.470 & 0.206 & 2.767 & 0.50 & 65.0 \\
\hline 78.150 & 0.202 & 2.586 & 0.52 & 65.0 \\
\hline 79.980 & 0.310 & 3.870 & 0.54 & 65.0 \\
\hline 83.680 & 0.228 & 2.728 & 0.56 & 65.0 \\
\hline 86.580 & 0.290 & 3.351 & 0.58 & 65.0 \\
\hline 89.990 & 0.260 & 2.894 & 0.60 & 65.0 \\
\hline 92.980 & 0.219 & 2.353 & 0.63 & 65.0 \\
\hline 95.860 & 0.326 & 3.401 & 0.64 & 65.0 \\
\hline 99.330 & 0.320 & 3.221 & 0.67 & 65.0 \\
\hline 102.200 & 0.287 & 2.812 & 0.68 & 65.0 \\
\hline 105.200 & 0.246 & 2.341 & 0.71 & 65.0 \\
\hline 108.000 & 0.206 & 1.908 & 0.73 & 65.0 \\
\hline 111.300 & 0.299 & 2.683 & 0.75 & 65.0 \\
\hline 113.600 & 0.302 & 2.658 & 0.77 & 65.0 \\
\hline 117.600 & 0.199 & 1.689 & 0.79 & 65.0 \\
\hline 119.500 & 0.230 & 1.922 & 0.81 & 65.0 \\
\hline 122.800 & 0.250 & 2.036 & 0.83 & 65.0 \\
\hline 125.800 & 0.261 & 2.073 & 0.85 & 65.0 \\
\hline 129.200 & 0.257 & 1.986 & 0.87 & 65.0 \\
\hline 132.100 & 0.225 & 1.699 & 0.89 & 65.0 \\
\hline 135.300 & 0.200 & 1.475 & 0.91 & 65.0 \\
\hline 137.600 & 0.241 & 1.748 & 0.93 & 65.1 \\
\hline 141.200 & 0.276 & 1.951 & 0.95 & 65.2 \\
\hline 144.100 & 0.332 & 2.302 & 0.97 & 65.2 \\
\hline 147.000 & 0.294 & 2.003 & 0.99 & 65.2 \\
\hline 149.800 & 0.348 & 2.321 & 1.01 & 65.2 \\
\hline 153.300 & 0.308 & 2.010 & 1.03 & 65.2 \\
\hline 156.100 & 0.397 & 2.546 & 1.05 & 65.2 \\
\hline 159.500 & 0.178 & 1.115 & 1.07 & 65.2 \\
\hline 161.800 & 0.175 & 1.082 & 1.09 & 65.1 \\
\hline 165.300 & 0.391 & 2.365 & 1.11 & 65.1 \\
\hline 168.100 & 0.393 & 2.338 & 1.13 & 65.2 \\
\hline 171.600 & 0.300 & 1.746 & 1.15 & 65.2 \\
\hline 174.000 & 0.363 & 2.085 & 1.17 & 65.1 \\
\hline 177.300 & 0.300 & 1.690 & 1.19 & 65.1 \\
\hline 180.700 & 0.418 & 2.314 & 1.21 & 65.1 \\
\hline 183.600 & 0.383 & 2.085 & 1.23 & 65.1 \\
\hline 187.000 & 0.128 & 0.685 & 1.25 & 65.1 \\
\hline 189.100 & 0.359 & 1.899 & 1.27 & 65.1 \\
\hline 192.700 & 0.309 & 1.601 & 1.29 & 65.1 \\
\hline 195.600 & 0.303 & 1.549 & 1.31 & 65.1 \\
\hline
\end{tabular}


RPP-5798, Rev. 0

\begin{tabular}{|c|c|c|c|c|}
\hline 199.000 & 0.427 & 2.147 & 1.33 & 65.1 \\
\hline 201.900 & 0.348 & .1 .725 & 1.35 & 65.1 \\
\hline 204.700 & 0.298 & 1.454 & 1.37 & 65.1 \\
\hline 207.600 & 0.274 & 1.318 & 1.39 & 65.2 \\
\hline 211.000 & 0.299 & 1.418 & 1.41 & 65.1 \\
\hline 213.900 & 0.337 & 1.578 & 1.43 & 65.2 \\
\hline 216.700 & 0.423 & 1.951 & 1.45 & 65.1 \\
\hline 219.600 & 0.376 & 1.713 & 1.47 & 65.1 \\
\hline 223.000 & 0.366 & 1.642 & 1.49 & 65.1 \\
\hline 225.900 & 0.460 & 2.038 & 1.51 & 65.0 \\
\hline 229.100 & 0.442 & 1.930 & 1.53 & 65.1 \\
\hline 231.600 & 0.512 & 2.212 & 1.55 & 65.0 \\
\hline 235.000 & 0.346 & 1.472 & 1.58 & 65.1 \\
\hline 237.900 & 0.381 & 1.603 & 1.59 & 65.1 \\
\hline 241.300 & 0.310 & 1.285 & 1.62 & 65.1 \\
\hline 243.600 & 0.488 & 2.004 & 1.63 & 65.1 \\
\hline 247.000 & 0.439 & 1.775 & 1.66 & 65.1 \\
\hline 249.900 & 0.414 & 1.658 & 1.67 & 65.1 \\
\hline 253.300 & 0.491 & 1.936 & 1.70 & 65.1 \\
\hline 256.200 & 0.369 & 1.439 & 1.71 & 65.1 \\
\hline 259.000 & 0.563 & 2.172 & 1.74 & 65.2 \\
\hline 261.900 & 0.460 & 1.757 & 1.75 & 65.2 \\
\hline 265.300 & 0.403 & 1.517 & 1.78 & 65.2 \\
\hline 268.800 & 0.578 & 2.152 & 1.80 & 65.2 \\
\hline 271.400 & 0.479 & 1.765 & 1.82 & 65.2 \\
\hline 274.500 & 0.477 & 1.739 & 1.84 & 65.2 \\
\hline 277.300 & 0.462 & 1.665 & 1.86 & 65.2 \\
\hline 280.800 & 0.539 & 1.920 & 1.88 & 65.2 \\
\hline 283.600 & 0.474 & 1.669 & 1.90 & 65.1 \\
\hline 286.500 & 0.585 & 2.040 & 1.92 & 65.1 \\
\hline 289.400 & 0.416 & 1.439 & 1.94 & 65.1 \\
\hline 292.800 & 0.577 & 1.972 & 1.96 & 65.2 \\
\hline 295.600 & 0.476 & 1.611 & 1.98 & 65.1 \\
\hline 298.900 & 0.576 & 1.927 & 2.00 & 65.1 \\
\hline 300.200 & 0.445 & 1.483 & 2.00 & 65.1 \\
\hline 298.700 & 0.450 & 1.508 & 2.02 & 65.1 \\
\hline 295.500 & 0.476 & 1.612 & 2.04 & 65.1 \\
\hline 292.600 & 0.368 & 1.256 & 2.06 & 65.1 \\
\hline 289.200 & 0.454 & 1.569 & 2.08 & 65.1 \\
\hline 286.900 & 0.463 & 1.614 & 2.10 & 65.1 \\
\hline 283.400 & 0.276 & 0.975 & 2.12 & 65.1 \\
\hline 280.600 & 0.412 & 1.469 & 2.14 & 65.1 \\
\hline 277.300 & 0.313 & 1.128 & 2.16 & 65.1 \\
\hline 274.500 & 0.379 & 1.379 & 2.18 & 65.1 \\
\hline 271.400 & 0.379 & 1.394 & 2.20 & 65.1 \\
\hline 268.600 & 0.268 & 0.998 & 2.22 & 65.1 \\
\hline 265.100 & 0.366 & 1.381 & 2.24 & 65.1 \\
\hline 262.300 & 0.309 & 1.176 & 2.26 & 65.1 \\
\hline 259.000 & 0.396 & 1.529 & 2.28 & 65.1 \\
\hline 256.400 & 0.339 & 1.322 & 2.30 & 65.1 \\
\hline 253.100 & 0.366 & 1.447 & 2.32 & 65.2 \\
\hline 250.300 & 0.288 & 1.150 & 2.34 & 65.1 \\
\hline 247.000 & 0.366 & 1.482 & 2.36 & 65.1 \\
\hline 244.400 & 0.280 & 1.147 & 2.38 & 65.1 \\
\hline 241.100 & 0.361 & 1.497 & 2.40 & 65.1 \\
\hline 238.100 & 0.311 & 1.306 & 2.42 & 65.1 \\
\hline 233.900 & 0.271 & 1.160 & 2.44 & 65.1 \\
\hline 231.600 & 0.302 & 1.302 & 2.46 & 65.1 \\
\hline 229.100 & 0.272 & 1.188 & 2.48 & 65.1 \\
\hline 225.700 & 0.177 & 0.784 & 2.50 & 65.1 \\
\hline 223.000 & 0.241 & 1.079 & 2.52 & 65.1 \\
\hline 220.000 & 0.311 & 1.414 & 2.54 & 65.0 \\
\hline 216.900 & 0.243 & 1.120 & 2.56 & 65.0 \\
\hline 214.400 & 0.278 & 1.298 & 2.58 & 65.0 \\
\hline 210.600 & 0.229 & 1.088 & 2.60 & 65.0 \\
\hline 207.200 & 0.281 & 1.355 & 2.63 & 65.0 \\
\hline 204.700 & 0.230 & 1.122 & 2.64 & 65.0 \\
\hline 201.900 & 0.267 & 1.323 & 2.67 & 65.0 \\
\hline 198.400 & 0.326 & 1.643 & 2.69 & 65.0 \\
\hline 195.600 & 0.245 & 1.254 & 2.71 & 65.0 \\
\hline
\end{tabular}


RPP-5798, Rev. 0

\begin{tabular}{|c|c|c|c|c|}
\hline $\begin{array}{l}192.300 \\
189.300\end{array}$ & $\begin{array}{l}0.218 \\
0.266\end{array}$ & $\begin{array}{l}1.133 \\
1.403\end{array}$ & $\begin{array}{l}2.73 \\
2.75\end{array}$ & $\begin{array}{l}65.0 \\
65.0\end{array}$ \\
\hline $\begin{array}{l}189.300 \\
186.600\end{array}$ & $\begin{array}{l}0.200 \\
0.279\end{array}$ & $\begin{array}{l}1.403 \\
1.497\end{array}$ & $\begin{array}{l}2.75 \\
2.77\end{array}$ & $\begin{array}{l}65.0 \\
65.0\end{array}$ \\
\hline $\begin{array}{l}186.600 \\
183.200\end{array}$ & 0.222 & 1.213 & 2.79 & 65.0 \\
\hline 180.500 & 0.128 & 0.710 & 2.81 & 65.0 \\
\hline 177.300 & 0.193 & 1.088 & 2.83 & 65.0 \\
\hline 174.400 & 0.235 & 1.349 & 2.84 & 65.0 \\
\hline 171.600 & 0.182 & 1.061 & 2.87 & 65.0 \\
\hline 168.500 & 0.228 & 1.352 & 2.89 & 65.0 \\
\hline 165.100 & 0.183 & 1.107 & 2.91 & 65.0 \\
\hline 162.400 & 0.172 & $1.06 !$ & 2.93 & 65.0 \\
\hline 159.200 & 0.193 & 1.213 & 2.95 & 65.0 \\
\hline 156.300 & 0.181 & 1.155 & 2.97 & 65.0 \\
\hline 152.900 & 0.196 & 1.282 & 2.99 & 65.0 \\
\hline 149.800 & 0.138 & 0.924 & 3.01 & 65.0 \\
\hline 147.000 & 0.146 & 0.992 & 3.03 & 65.0 \\
\hline 144.700 & 0.173 & 1.195 & 3.05 & 65.0 \\
\hline 140.900 & 0.191 & 1.354 & 3.07 & 65.0 \\
\hline 138.000 & 0.172 & 1.247 & 3.09 & 65.0 \\
\hline 135.000 & 0.125 & 0.923 & 3.11 & 65.0 \\
\hline 132.100 & 0.035 & 0.269 & 3.13 & 65.0 \\
\hline 128.700 & 0.113 & 0.876 & 3.15 & 65.0 \\
\hline 126.000 & 0.129 & 1.026 & 3.17 & 65.0 \\
\hline 122.400 & 0.121 & 0.990 & 3.19 & 65.0 \\
\hline 119.500 & 0.103 & 0.864 & 3.21 & 65.0 \\
\hline 116.700 & 0.090 & 0.768 & 3.23 & 65.0 \\
\hline 113.200 & 0.108 & 0.949 & 3.25 & 65.0 \\
\hline 110.400 & 0.187 & 1.693 & 3.27 & 65.0 \\
\hline 108.000 & 0.169 & 1.563 & 3.29 & 65.0 \\
\hline 105.300 & 0.098 & 0.933 & 3.31 & 65.0 \\
\hline 101.800 & 0.117 & 1.144 & 3.33 & 65.0 \\
\hline 98.990 & 0.137 & 1.388 & 3.35 & 65.0 \\
\hline 95.520 & 0.163 & 1.706 & 3.37 & 65.0 \\
\hline 93.040 & 0.060 & 0.645 & 3.39 & 65.0 \\
\hline 89.700 & 0.100 & 1.117 & 3.41 & 64.9 \\
\hline 86.840 & 0.091 & 1.043 & 3.43 & 64.9 \\
\hline 83.410 & 0.174 & 2.091 & 3.45 & 65.0 \\
\hline 80.500 & 0.151 & 1.870 & 3.47 & 65.0 \\
\hline 77.540 & 0.154 & 1.991 & 3.49 & 65.0 \\
\hline 74.740 & 0.109 & 1.455 & 3.51 & 65.0 \\
\hline 71.250 & 0.127 & 1.788 & 3.53 & 65.0 \\
\hline 68.410 & 0.173 & 2.534 & 3.55 & 65.0 \\
\hline 65.360 & 0.108 & 1.658 & 3.58 & 65.0 \\
\hline 62.600 & 0.113 & 1.802 & 3.59 & 65.0 \\
\hline 59.190 & 0.091 & 1.541 & 3.62 & 65.0 \\
\hline 56.270 & 0.108 & 1.925 & 3.63 & 65.0 \\
\hline 52,840 & 0.089 & 1.676 & 3.66 & 65.0 \\
\hline 50.460 & 0.109 & 2.151 & 3.67 & 65.0 \\
\hline 47.060 & 0.082 & 1.739 & 3.70 & 65.0 \\
\hline 44.200 & 0.075 & 1.688 & 3.71 & 65.0 \\
\hline 40.720 & 0.071 & 1.749 & 3.74 & 65.0 \\
\hline 37.950 & 0.102 & 2.683 & 3.75 & 65.0 \\
\hline 35.000 & 0.084 & 2.398 & 3.78 & 65.0 \\
\hline 31.530 & 0.076 & 2.397 & 3.80 & 65.0 \\
\hline 28.650 & 0.063 & 2.189 & 3.82 & 65.0 \\
\hline 25.180 & 0.072 & 2.841 & 3.84 & 65.0 \\
\hline 22.780 & 0.066 & 2.904 & 3.86 & 65.0 \\
\hline 19.420 & 0.089 & 4.578 & 3.88 & 65.0 \\
\hline 16.600 & 0.077 & 4.622 & 3.90 & 65.0 \\
\hline 13.150 & 0.069 & 5.238 & 3.92 & 65.0 \\
\hline 10.350 & 0.074 & 7.167 & 3.94 & 65.0 \\
\hline 7.396 & 0.045 & 6.108 & 3.96 & 65.0 \\
\hline 4.556 & 0.017 & 3.808 & 3.98 & 65.0 \\
\hline 1.048 & 0.033 & 31.100 & 4.00 & 65.0 \\
\hline
\end{tabular}


RPP-5798, Rev. 0

VISCOȘITY ANALYSIS OF

DILUTION LEVEL "-" (60 g solids/L)

AT AMBIENT TEMPERATURE 
RPP-5798, Rev. 0

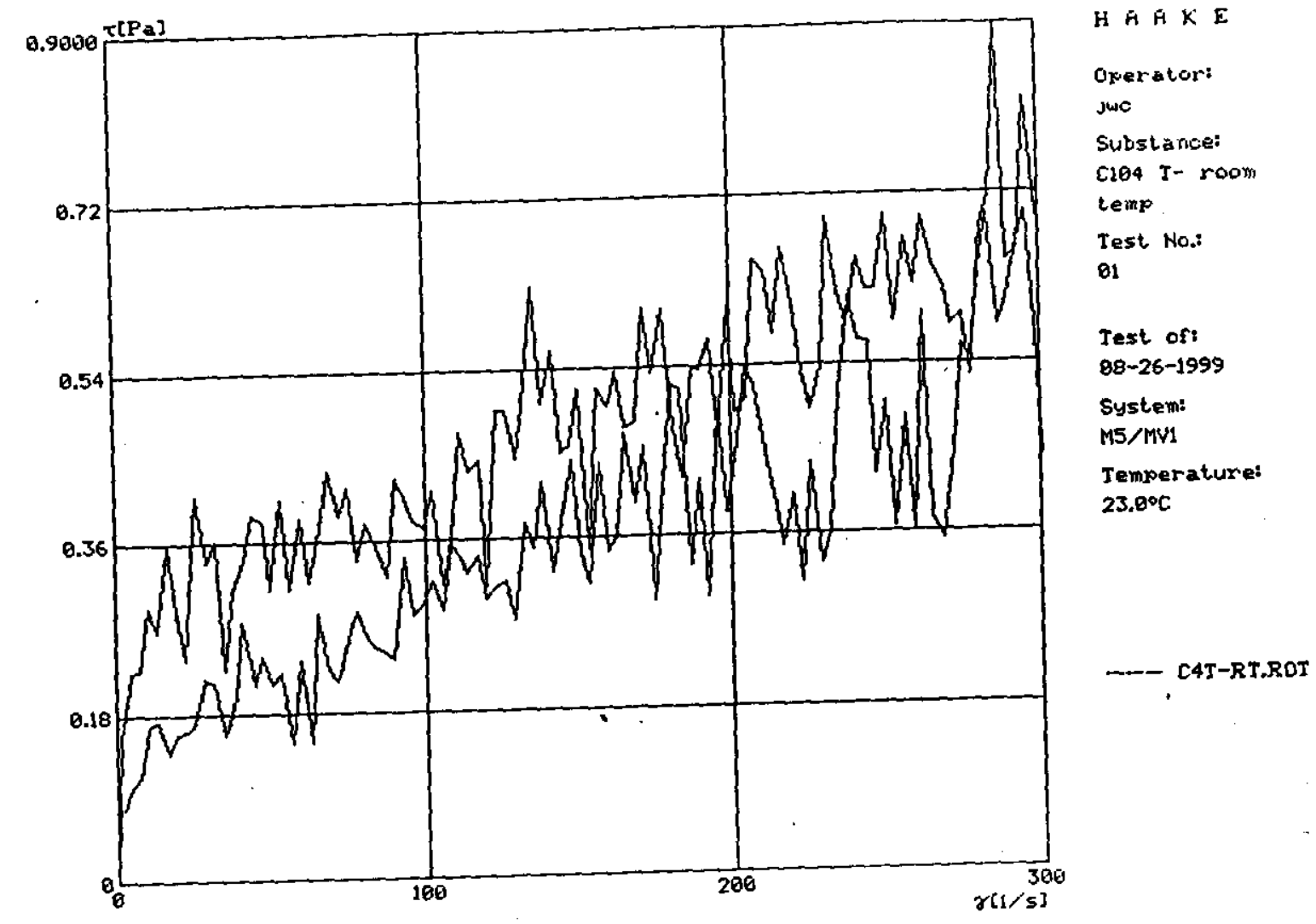

HAGKE ROE 3.0 .3

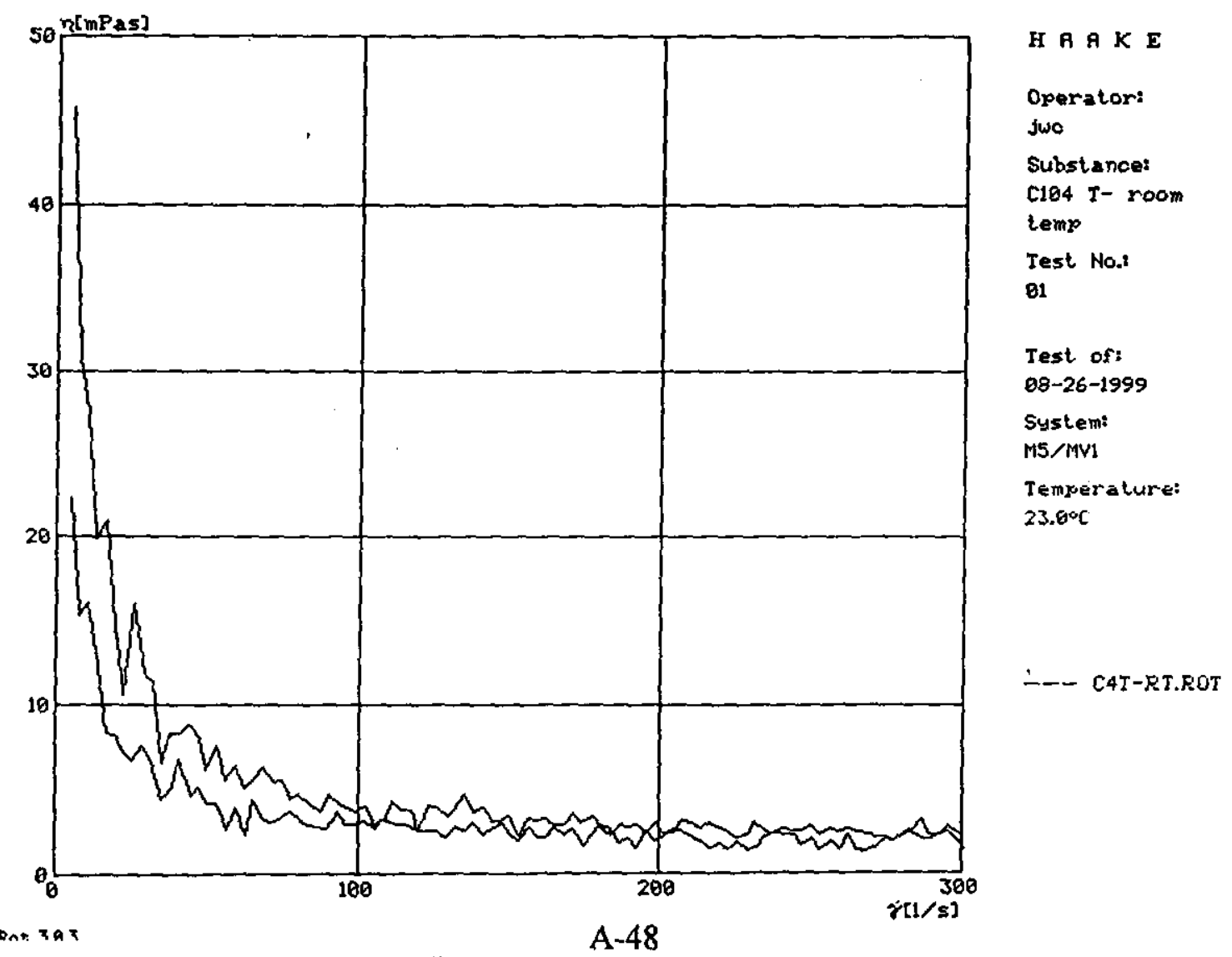


RPP-5798, Rev. 0

\begin{tabular}{|c|c|c|c|c|}
\hline$\tau$ [Pa] & $\gamma\left[\mathbf{s}^{-1}\right]$ & $\eta[\mathrm{mPas}]$ & t[min] & Temp $\left[{ }^{\circ} \mathrm{C}\right]$ \\
\hline 0.000 & 0.000 & 0.000 & 0.00 & 23.8 \\
\hline 1.468 & 0.158 & 107.500 & 0.02 & 23.8 \\
\hline 4.899 & 0.224 & 45.810 & 0.04 & 23.8 \\
\hline 7.320 & 0.226 & 30.910 & 0.06 & 23.8 \\
\hline 10.620 & 0.293 & 27.550 & 0.08 & 23.8 \\
\hline 13.500 & 0.268 & 19.890 & 0.10 & 23.8 \\
\hline 16.980 & 0.358 & 21.090 & 0.12 & 23.8 \\
\hline 19.750 & 0.289 & 14.640 & 0.14 & 23.8 \\
\hline 22.300 & 0.238 & 10.660 & 0.15 & 23.8 \\
\hline 25.540 & 0.411 & 16.090 & 0.18 & 23.8 \\
\hline 29.010 & 0.342 & 11.770 & 0.20 & 23.8 \\
\hline 31.870 & 0.363 & 11.390 & 0.22 & 23.8 \\
\hline 34.560 & 0.228 & 6.604 & 0.23 & 23.8 \\
\hline 37.460 & 0.311 & 8.313 & 0.26 & 23.8 \\
\hline 41.080 & 0.344 & 8.362 & 0.28 & 23.8 \\
\hline 43.980 & 0.390 & 8.875 & 0.30 & 23.8 \\
\hline 47.460 & 0.382 & 8.053 & 0.32 & 23.8 \\
\hline 49.850 & 0.311 & 6.237 & 0.34 & 23.8 \\
\hline 53.220 & 0.406 & 7.635 & 0.36 & 23.8 \\
\hline 56.120 & 0.310 & 5.532 & 0.38 & 23.8 \\
\hline 59.590 & 0.387 & 6.486 & 0.40 & 23.8 \\
\hline 62.140 & 0.318 & 5.110 & 0.42 & 23.9 \\
\hline 65.340 & 0.374 & 5.727 & 0.44 & 23.8 \\
\hline 68.200 & 0.435 & 6.380 & 0.46 & 23.9 \\
\hline 71.650 & 0.389 & 5.434 & 0.48 & 23.8 \\
\hline 74.510 & 0.418 & 5.612 & 0.50 & 23.8 \\
\hline 77.500 & 0.340 & 4.383 & 0.52 & 23.9 \\
\hline 80.310 & 0.380 & 4.736 & 0.54 & 23.9 \\
\hline 83.760 & 0.353 & 4.220 & 0.56 & 23.8 \\
\hline 87.210 & 0.324 & 3.711 & 0.59 & 23.8 \\
\hline 90.010 & 0.428 & 4.751 & 0.60 & 23.8 \\
\hline 92.980 & 0.406 & 4.370 & 0.63 & 23.9 \\
\hline 95.860 & 0.382 & 3.983 & 0.64 & 23.8 \\
\hline 99.390 & 0.371 & 3.737 & 0.67 & 23.9 \\
\hline 102.200 & 0.413 & 4.043 & 0.68 & 23.9 \\
\hline 105.200 & 0.313 & 2.977 & 0.71 & 23.9 \\
\hline 108.000 & 0.361 & 3.341 & 0.73 & 23.9 \\
\hline 111.300 & 0.474 & 4.257 & 0.75 & 23.9 \\
\hline 113.800 & 0.433 & 3.807 & 0.77 & 23.9 \\
\hline 117.200 & 0.444 & 3.789 & 0.79 & 23.9 \\
\hline 119.500 & 0.311 & 2.601 & 0.81 & 23.9 \\
\hline 122.900 & 0.496 & 4.035 & 0.83 & 23.9 \\
\hline 125.800 & 0.498 & 3.955 & 0.85 & 23.9 \\
\hline 129.200 & 0.447 & 3.462 & 0.87 & 23.9 \\
\hline 131.500 & 0.503 & 3.826 & 0.89 & 23.9 \\
\hline 135.000 & 0.626 & 4.635 & 0.91 & 23.9 \\
\hline 137.800 & 0.503 & 3.651 & 0.93 & 23.9 \\
\hline 141.200 & 0.559 & 3.954 & 0.95 & 23.9 \\
\hline 144.100 & 0.452 & 3.134 & 0.97 & 23.9 \\
\hline 147.000 & 0.459 & 3.122 & 0.99 & 23.9 \\
\hline 149.800 & 0.517 & 3.453 & 1.01 & 24.0 \\
\hline 153.300 & 0.347 & 2.263 & 1.03 & 23.9 \\
\hline 156.100 & 0.517 & 3.314 & 1.05 & 23.9 \\
\hline 159.400 & 0.500 & 3.140 & 1.07 & 23.9 \\
\hline 161.800 & 0.537 & 3.317 & 1.09 & 23.9 \\
\hline 165.300 & 0.478 & 2,890 & 1.11 & 23.9 \\
\hline 168.100 & 0.483 & 2.872 & 1.13 & 23.9 \\
\hline 171.600 & 0.602 & 3.506 & 1.15 & 23.9 \\
\hline 173.800 & 0.534 & 3.071 & 1.17 & 23.9 \\
\hline 177.300 & 0.599 & 3.380 & 1.19 & 23.9 \\
\hline 180.700 & 0.475 & 2.628 & 1.21 & 24.0 \\
\hline 183.600 & 0.422 & 2.296 & 1.23 & 23.9 \\
\hline 187.000 & 0.540 & 2.888 & 1.25 & 23.9 \\
\hline 189.300 & 0.537 & 2.836 & 1.27 & 23.9 \\
\hline 192.700 & 0.567 & 2.945 & 1.29 & 23.9 \\
\hline 195.600 & 0.471 & 2.409 & 1.31 & 23.9 \\
\hline
\end{tabular}


RPP-5798, Rev. 0

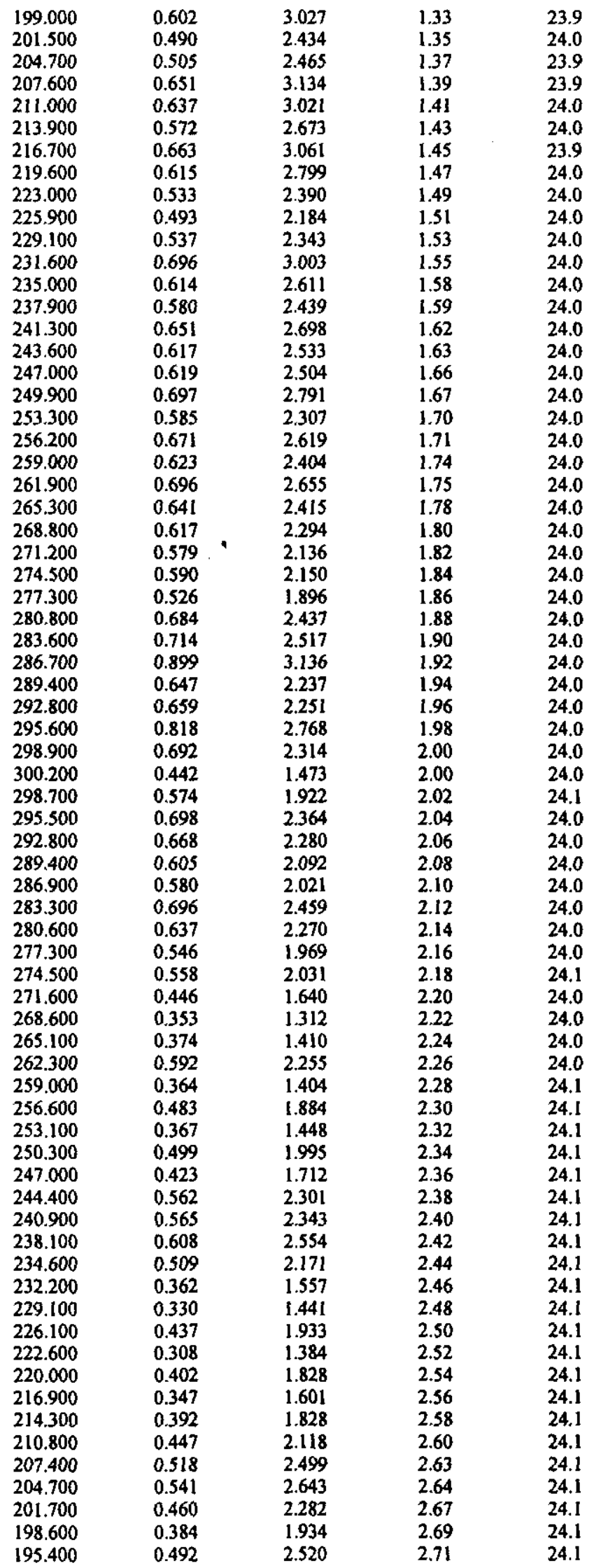


RPP-5798, Rev. 0

\begin{tabular}{|c|c|c|c|c|}
\hline 192.500 & 0.295 & 1.534 & 2.73 & 24.1 \\
\hline 189.300 & 0.420 & 2.217 & 2.75 & 24.1 \\
\hline 186.600 & 0.328 & 1.760 & 2.77 & 24.1 \\
\hline 183.200 & 0.516 & 2.814 & 2.79 & 24.1 \\
\hline 180.500 & 0.523 & 2.895 & 2.81 & 24.1 \\
\hline 177.300 & 0.417 & 2.353 & 2.83 & 24.1 \\
\hline 174.600 & 0.294 & 1.683 & 2.85 & 24.1 \\
\hline 171.200 & 0.457 & 2.672 & 2.87 & 24.1 \\
\hline 168.500 & 0.397 & 2.355 & 2.89 & 24.1 \\
\hline 165.100 & 0.471 & 2.851 & 2.91 & 24.1 \\
\hline 162.400 & 0.358 & 2.205 & 2.93 & 24.1 \\
\hline 159.400 & 0.345 & 2.164 & 2.95 & 24.1 \\
\hline 156.300 & 0.440 & 2.817 & 2.97 & 24.1 \\
\hline 152.900 & 0.312 & 2.043 & 2.99 & 24.1 \\
\hline 150.000 & 0.342 & 2.280 & 3.01 & 24.1 \\
\hline 147.200 & 0.442 & 3.002 & 3.03 & 24.1 \\
\hline 144.300 & 0.380 & 2.633 & 3.05 & 24.1 \\
\hline 140.900 & 0.325 & 2.304 & 3.07 & 24.1 \\
\hline 138.000 & 0.420 & 3.040 & 3.09 & 24.1 \\
\hline 135.000 & 0.349 & 2.587 & 3.11 & 24.1 \\
\hline 132.100 & 0.377 & 2.854 & 3.13 & 24.1 \\
\hline 128.900 & 0.274 & 2.127 & 3.15 & 24.1 \\
\hline 125.800 & 0.317 & 2.516 & 3.17 & 24.1 \\
\hline 122.400 & 0.309 & 2.521 & 3.19 & 24.1 \\
\hline 119.500 & 0.299 & 2.499 & 3.21 & 24.1 \\
\hline 116.700 & 0.344 & 2.949 & 3.23 & 24.1 \\
\hline 113.200 & 0.325 & 2.867 & 3.25 & 24.1 \\
\hline 110.800 & 0.344 & 3.104 & 3.27 & 24.1 \\
\hline 108.000 & 0.353 & 3.272 & 3.29 & 24.1 \\
\hline 105.400 & 0.287 & 2.722 & 3.31 & 24.1 \\
\hline 101.900 & 0.319 & 3.125 & 3.33 & 24.1 \\
\hline 99.020 & 0.294 & 2.968 & 3.35 & 24.1 \\
\hline 95.560 & 0.281 & 2.937 & 3.37 & 24.1 \\
\hline 93.250 & 0.342 & 3.668 & 3.39 & 24.1 \\
\hline 89.760 & 0.235 & 2.621 & 3.41 & 24.1 \\
\hline 86.840 & 0.243 & 2.802 & 3.43 & 24.1 \\
\hline 83.430 & 0.250 & 2.990 & 3.45 & 24.1 \\
\hline 81.110 & 0.257 & 3.163 & 3.47 & 24.1 \\
\hline 77.640 & 0.286 & 3.682 & 3.49 & 24.1 \\
\hline 75.060 & 0.259 & 3.443 & 3.51 & 24.1 \\
\hline 71.370 & 0.214 & 2.999 & 3.53 & 24.1 \\
\hline 68.680 & 0.224 & 3.261 & 3.55 & 24.1 \\
\hline 64.900 & 0.285 & 4.390 & 3.58 & 24.1 \\
\hline 62.960 & 0.148 & 2.357 & 3.59 & 24.1 \\
\hline 59.360 & 0.237 & 3.988 & 3.61 & 24.1 \\
\hline 56.400 & 0.149 & 2.639 & 3.63 & 24.2 \\
\hline 52.900 & 0.223 & 4.210 & 3.66 & 24.1 \\
\hline 50.150 & 0.212 & 4.225 & 3.67 & 24.1 \\
\hline 47.040 & 0.241 & 5.132 & 3.69 & 24.1 \\
\hline 44.470 & 0.211 & 4.740 & 3.71 & 24.2 \\
\hline 40.320 & 0.277 & 6.880 & 3.74 & 24.2 \\
\hline 38.410 & 0.198 & 5.162 & 3.75 & 24.2 \\
\hline 35.020 & 0.157 & 4.475 & 3.78 & 24.2 \\
\hline 31.590 & 0.213 & 6.736 & 3.80 & 24.2 \\
\hline 28.940 & 0.218 & 7.543 & 3.81 & 24.1 \\
\hline 25.290 & 0.170 & 6.736 & 3.84 & 24.2 \\
\hline 22.820 & 0.162 & 7.091 & 3.86 & 24.2 \\
\hline 19.500 & 0.160 & 8.188 & 3.88 & 24.2 \\
\hline 16.660 & 0.140 & 8.375 & 3.90 & 24.2 \\
\hline 13.190 & 0.173 & 13.090 & 3.92 & 24.2 \\
\hline 10.430 & 0.167 & 16.040 & 3.94 & 24.1 \\
\hline 7.396 & 0.113 & 15.310 & 3.96 & 24.1 \\
\hline 4.537 & 0.102 & 22.450 & 3.98 & 24.1 \\
\hline 1.087 & 0.074 & 67.680 & 4.00 & 24.2 \\
\hline
\end{tabular}


RPP-5798, Rev. 0

\section{VISCOSITY ANALYSIS OF}

DILUTION LEVEL "-" (60 g solids/L) AT $45^{\circ} \mathrm{C}$ 
RPP-5798, Rev. 0

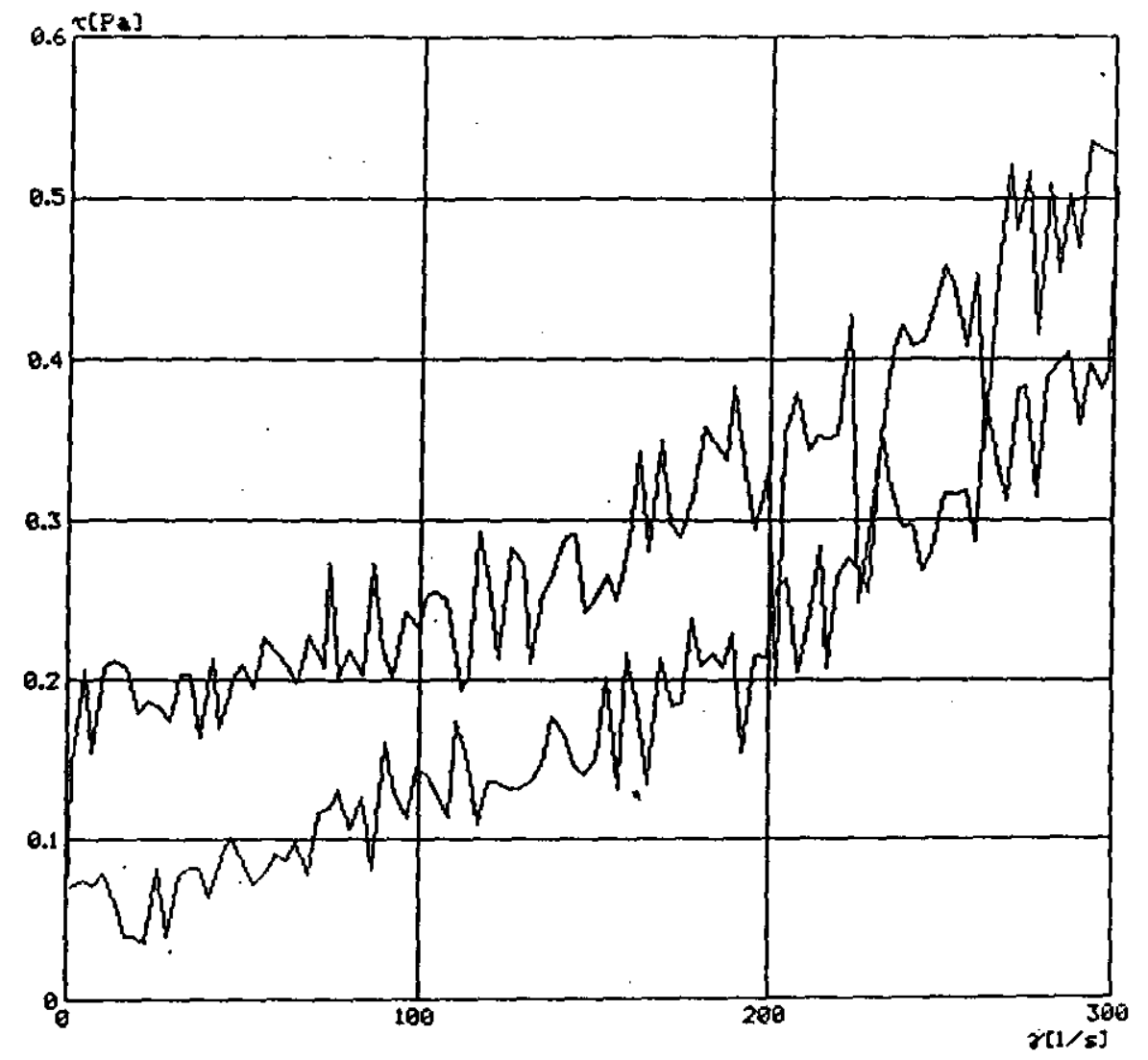
HAFKE
Operator:
jwe
Substance:
C104 I- room
tome- $45 \mathrm{C}$
Test No: $45 C$ JFO
at
$1 / 27 / \infty$

Test of:

08-26-1999

System:

MS/MYI

Temperature:

$23.0^{\circ} \mathrm{C}$

- C4T-45.ROT

WGEKF Dor: 3 ด

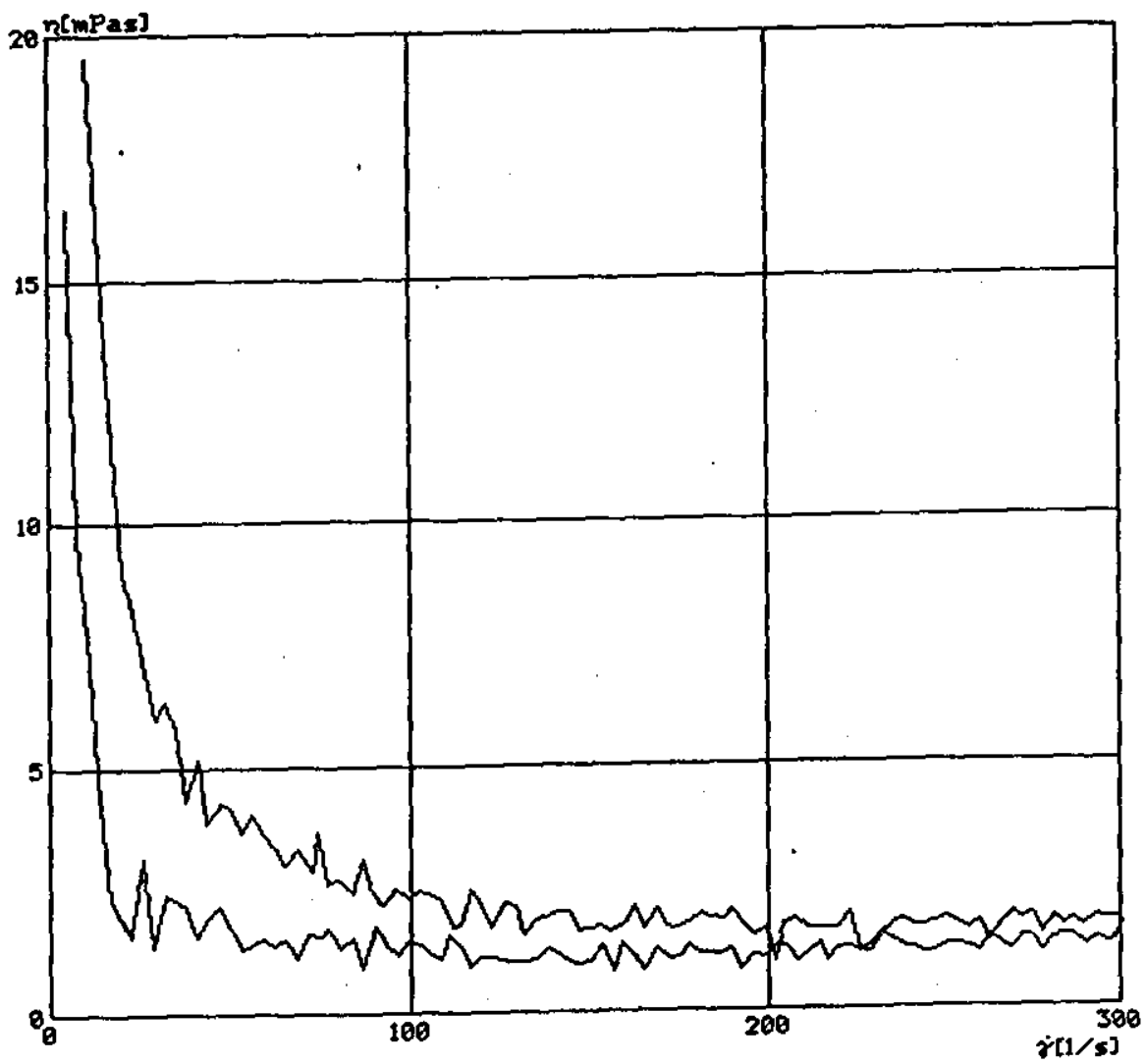

HคA $\mathrm{K} E$

operator:

twe

Substance:

C104 I- now

tommen 45C JFe

Test No.: i $B \geqslant 0$

ol

Test of:

88-26-1999

System:

MS/MYI

Temperature:

$23.0^{\circ} \mathrm{C}$

C- C4T-45.ROT 
RPP-5798, Rev. 0

\begin{tabular}{|c|c|c|c|c|}
\hline$\tau[\mathbf{P a}]$ & $\gamma\left[\mathbf{s}^{-1}\right]$ & $\eta[m P a s]$ & $t\{m i n\}$ & Temp $\left.\left.\right|^{\circ} \mathrm{C}\right]$ \\
\hline 0.000 & 0.053 & 0.000 & 0.00 & 44.9 \\
\hline 1.506 & 0.147 & 97.790 & 0.02 & 44.9 \\
\hline 4.918 & 0.205 & 41.750 & 0.04 & 44.9 \\
\hline 7.262 & 0.154 & 21.250 & 0.06 & 44.9 \\
\hline 10.640 & 0.208 & 19.580 & 0.08 & 44.9 \\
\hline 13.060 & 0.212 & 16.210 & 0.10 & 44.9 \\
\hline 16.950 & 0.207 & 12.210 & 0.12 & 44.9 \\
\hline 19.960 & 0.178 & 8.925 & 0.14 & 44.9 \\
\hline 22.680 & 0.187 & 8.242 & 0.16 & 44.9 \\
\hline 25.560 & 0.184 & 7.199 & 0.18 & 44.9 \\
\hline 28.990 & 0.174 & 6.016 & 0.20 & 44.9 \\
\hline 31.910 & 0.203 & 6.370 & 0.22 & 44.9 \\
\hline 34.940 & 0.203 & 5.812 & 0.24 & 44.9 \\
\hline 37.820 & 0.164 & 4.339 & 0,26 & 44.9 \\
\hline 41.000 & 0.214 & 5.212 & 0.28 & 44.9 \\
\hline 43.570 & 0.170 & 3.893 & 0.29 & 44.9 \\
\hline 47.200 & 0.203 & 4.308 & 0.32 & 44.9 \\
\hline 49.790 & 0.209 & 4.205 & 0.34 & 44.9 \\
\hline 53.220 & 0.196 & 3.685 & 0.36 & 44.9 \\
\hline 56.040 & 0.226 & 4.026 & 0.38 & 44.9 \\
\hline 59.240 & 0.216 & 3.652 & 0.40 & 44.9 \\
\hline 62.080 & 0.209 & 3.371 & 0.42 & 44.9 \\
\hline 65.270 & 0.199 & 3.042 & 0.44 & 44.9 \\
\hline 68.200 & 0.227 & 3.324 & 0.46 & 44.9 \\
\hline 72.360 & 0.207 & 2.864 & 0.48 & 44.9 \\
\hline 74.170 & 0.273 & 3.680 & 0.50 & 44.9 \\
\hline 77.010 & 0.201 & 2.607 & 0.52 & 44.9 \\
\hline 79.940 & 0.218 & 2.723 & 0.54 & 44.9 \\
\hline 84.140 & 0.203 & 2.408 & 0.56 & 44.9 \\
\hline 86.790 & 0.273 & 3.145 & 0.58 & 44.9 \\
\hline 89.740 & 0.218 & 2.428 & 0.60 & 44.9 \\
\hline 92.370 & 0.201 & 2.174 & 0.62 & 44.9 \\
\hline 95.670 & 0.242 & 2.527 & 0.64 & 44.9 \\
\hline 99.270 & 0.233 & 2.345 & 0.67 & 44.9 \\
\hline 102.000 & 0.252 & 2.473 & 0.68 & 44.9 \\
\hline 105.000 & 0.255 & 2.423 & 0.70 & 44.9 \\
\hline 107.700 & 0.250 & 2.323 & 0.72 & 44.9 \\
\hline 112.100 & 0.194 & 1.730 & 0.75 & 44.9 \\
\hline 113.800 & 0.203 & 1,782 & 0.76 & 44.9 \\
\hline 116.800 & 0.292 & 2.502 & 0.79 & 44.9 \\
\hline 119.300 & 0.260 & 2.176 & 0.81 & 44.9 \\
\hline 122.400 & 0.213 & 1.739 & 0.83 & 44.9 \\
\hline 125.600 & 0.281 & 2.241 & 0.85 & 44.9 \\
\hline 129.200 & 0.272 & 2.105 & 0.87 & 44.9 \\
\hline 131.500 & 0.210 & 1.597 & 0.88 & 44.9 \\
\hline 135.000 & 0.255 & 1.886 & 0.91 & 45.0 \\
\hline 137.800 & 0.266 & 1.932 & 0.93 & 44.9 \\
\hline 141.200 & 0.289 & 2.043 & 0.95 & 44.9 \\
\hline 144.100 & 0.291 & 2.022 & 0.97 & 44.9 \\
\hline 147.000 & 0.242 & 1.648 & 0.99 & 44.9 \\
\hline 149.800 & 0.249 & 1.661 & 1.01 & 44.9 \\
\hline 153.300 & 0.264 & 1.725 & 1.03 & 44.9 \\
\hline 156.100 & 0.250 & 1.600 & 1.05 & 44.9 \\
\hline 159.500 & 0.286 & 1.791 & 1.07 & 44.9 \\
\hline 161.800 & 0.341 & 2.110 & 1.09 & 44.9 \\
\hline 165.300 & 0.280 & 1.692 & 1.11 & 44.9 \\
\hline 168.100 & 0.350 & 2.079 & 1.13 & 44.9 \\
\hline 171.600 & 0.297 & 1.729 & 1.15 & 44.9 \\
\hline 173.800 & 0.289 & 1.660 & 1.17 & 44.9 \\
\hline 177.300 & 0.314 & 1.769 & 1.19 & 44.9 \\
\hline 180.700 & 0.359 & 1.989 & 1.21 & 44.9 \\
\hline 183.600 & 0.349 & 1.899 & 1.23 & 44.9 \\
\hline 187.000 & 0.338 & 1.806 & 1.25 & 44.9 \\
\hline 189.300 & 0.384 & 2.026 & 1.27 & 44.9 \\
\hline 192.700 & 0.329 & 1.706 & 1.29 & 45.0 \\
\hline 195.600 & 0.294 & 1.502 & 1.31 & 44.9 \\
\hline
\end{tabular}


RPP-5798, Rev. 0

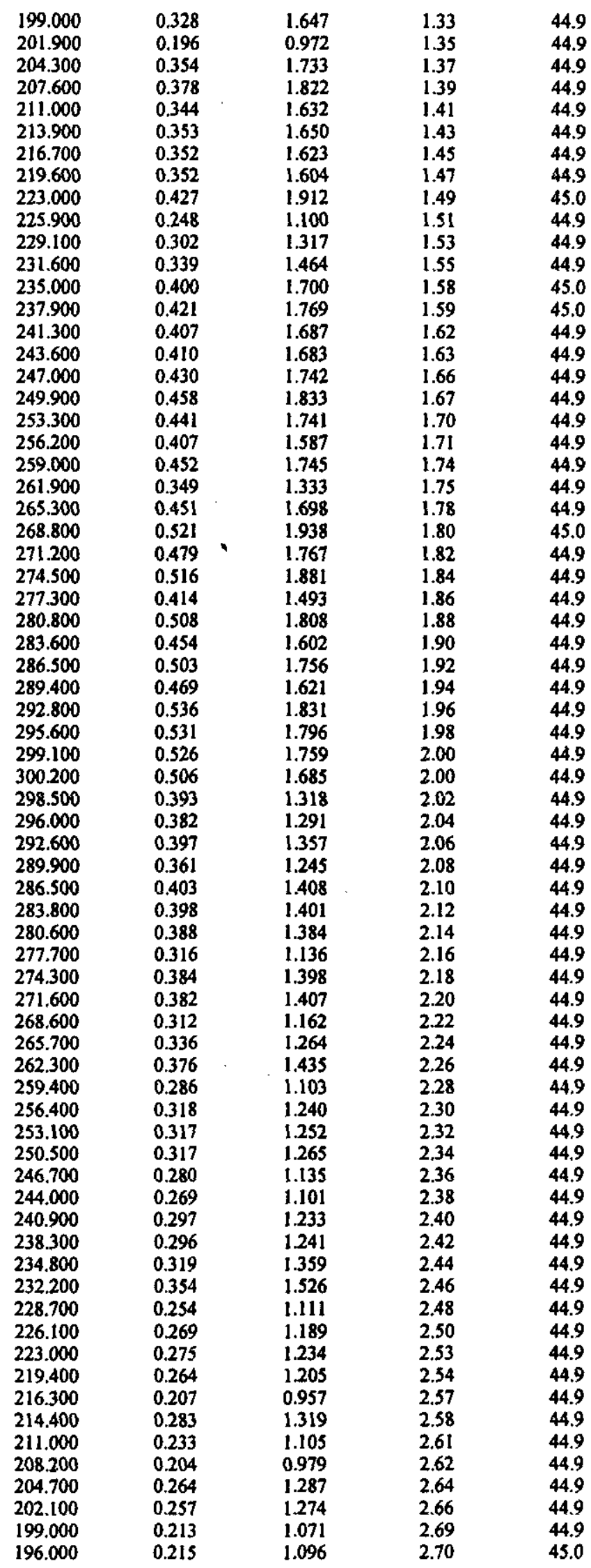


RPP-5798, Rev. 0

\begin{tabular}{|c|c|c|c|c|}
\hline 192.300 & 0.154 & 0.803 & 2.73 & 44.9 \\
\hline 189.700 & 0.229 & 1.206 & 2.74 & 44.9 \\
\hline 186.800 & 0.207 & 1.109 & 2.77 & 45.0 \\
\hline 183.800 & 0.217 & 1.182 & 2.78 & 45.0 \\
\hline 180.300 & 0.209 & 1.159 & 2.81 & 44.9 \\
\hline 177.800 & 0.238 & 1.341 & 2.82 & 44.9 \\
\hline 174.400 & 0.184 & 1.057 & 2.85 & 45.0 \\
\hline 171.900 & 0.183 & 1.062 & 2.87 & 44.9 \\
\hline 168.500 & 0.214 & 1.269 & 2.89 & 45.0 \\
\hline 165.100 & 0.135 & 0.815 & 2.91 & 44.9 \\
\hline 162.200 & 0.180 & 1.111 & 2.93 & 44.9 \\
\hline 159.000 & 0.217 & 1.364 & 2.95 & 44.9 \\
\hline 156.500 & 0.131 & 0.840 & 2.97 & 44.9 \\
\hline 152.900 & 0.202 & 1.320 & 2.99 & 44.9 \\
\hline 150.000 & 0.150 & 1.003 & 3.01 & 44.9 \\
\hline 146.600 & 0.142 & 0.966 & 3.03 & 45.0 \\
\hline 144.300 & 0.147 & 1.021 & 3.05 & 45.0 \\
\hline$[40.900$ & 0.165 & 1.171 & 3.07 & 45.0 \\
\hline 138.000 & 0.177 & 1.284 & 3.09 & 44.9 \\
\hline 134.600 & 0.147 & 1.094 & 3.11 & 45.0 \\
\hline 132.100 & 0.137 & 1.039 & 3.13 & 44.9 \\
\hline 128.900 & 0.132 & 1.023 & 3.15 & 45.0 \\
\hline 126.000 & 0.133 & 1.053 & 3.17 & 45.0 \\
\hline 122.600 & 0.136 & 1.107 & 3.19 & 44.9 \\
\hline 119.700 & 0.137 & 1.141 & 3.21 & 44.9 \\
\hline 116.700 & 0.109 & 0.937 & 3.23 & 44.9 \\
\hline 134.000 & 0.148 & 1.299 & 3.25 & 44.9 \\
\hline 110.400 & 0.175 & 1.585 & 3.27 & 44.9 \\
\hline 108.400 & 0.114 & 1.056 & 3.29 & 44.9 \\
\hline 104.900 & 0.129 & 1.230 & 3.31 & 44.9 \\
\hline 102.400 & 0.139 & 1.355 & 3.33 & 44.9 \\
\hline 98.990 & 0.145 & 1.465 & 3.35 & 44.9 \\
\hline 96.070 & 0.114 & 1.183 & 3.37 & 44.9 \\
\hline 92.620 & 0.129 & 1.396 & 3.39 & 44.9 \\
\hline 90.160 & 0.161 & 1.784 & 3.41 & 44.9 \\
\hline 86.830 & 0.081 & 0.927 & 3.43 & 44.9 \\
\hline 83.990 & 0.128 & 1.518 & 3.45 & 44.9 \\
\hline 80.540 & 0.106 & 1.319 & 3.47 & 44.9 \\
\hline 77.160 & 0.133 & 1.717 & 3.50 & 44.9 \\
\hline 74.660 & 0.119 & 1.595 & 3.51 & 44.9 \\
\hline 71.310 & 0.118 & 1.647 & 3.54 & 44.9 \\
\hline 68.430 & 0.078 & 1.136 & 3.55 & 44.9 \\
\hline 64.980 & 0.098 & 1.515 & 3.58 & 44.9 \\
\hline 62.390 & 0.087 & 1.393 & 3.59 & 44.9 \\
\hline 59.170 & 0.092 & 1.547 & 3.62 & 44.9 \\
\hline 56.270 & 0.080 & 1.416 & 3.63 & 44.9 \\
\hline 52.860 & 0.071 & 1.339 & 3.66 & 44.9 \\
\hline 50.000 & 0.085 & 1.705 & 3.67 & 44.9 \\
\hline 47.040 & 0.102 & 2.161 & 3.70 & 44.9 \\
\hline 44.180 & 0.088 & 1.993 & 3.72 & 44.9 \\
\hline 40.750 & 0.064 & 1.581 & 3.74 & 44.9 \\
\hline 37.890 & 0.082 & 2.173 & 3.76 & 44.9 \\
\hline 34.730 & 0.082 & 2.347 & 3.78 & 44.9 \\
\hline 32.100 & 0.078 & 2.421 & 3.80 & 44.9 \\
\hline 28.690 & 0.039 & 1.368 & 3.82 & 44.9 \\
\hline 25.830 & 0.082 & 3.168 & 3.84 & 44.9 \\
\hline 22.300 & 0.035 & 1.565 & 3.86 & 44.9 \\
\hline 19.820 & 0.040 & 1.995 & 3,88 & 44.9 \\
\hline 16.580 & 0.039 & 2.350 & 3.90 & 44.9 \\
\hline 13.720 & 0.059 & 4.272 & 3.92 & 44.9 \\
\hline 10.310 & 0.080 & 7.724 & 3.94 & 44.9 \\
\hline 7.415 & 0.072 & 9.678 & 3.96 & 44.9 \\
\hline 4.499 & 0.074 & 16.490 & 3.98 & 44.9 \\
\hline 1.658 & 0.070 & 42.050 & 4.00 & 44.9 \\
\hline
\end{tabular}


RPP-5798, Rev. 0

\section{VISCOṠITY ANALYSIS OF}

DILUTION LEVEL "-" (60 g solids/L) AT $65^{\circ} \mathrm{C}$ 
RPP-5798, Rev. 0

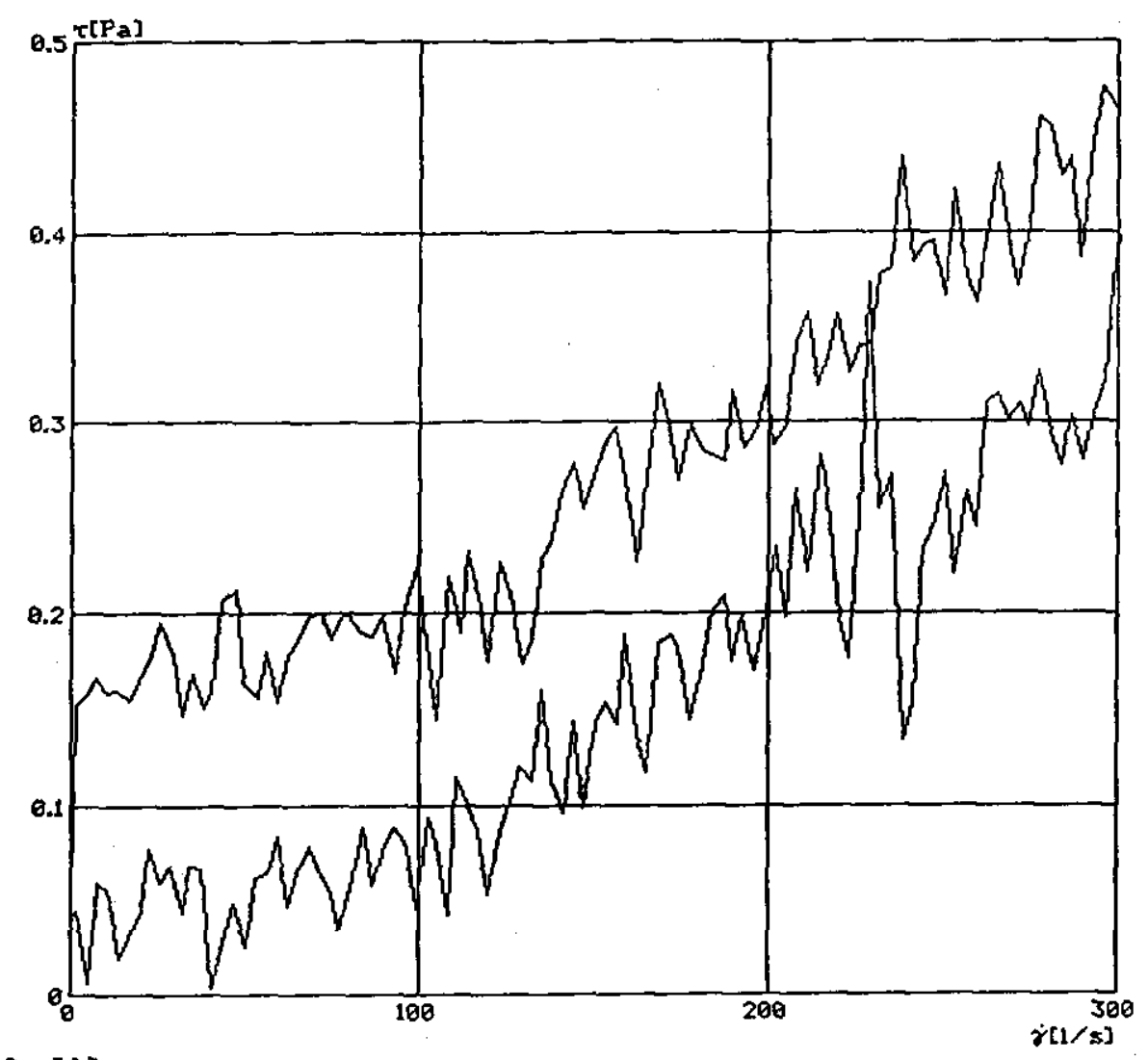

H ค ค $K E$

Operator:

Jwe

Substance:

C104 T- $65 \mathrm{C}$

Test No.:

घ1

Test of:

88-26-1999

System:

MS/MV1

Temperature:

$23.0^{\circ} \mathrm{C}$

D-D C4T-65.ROT

HFAKE RET 3.0 .3

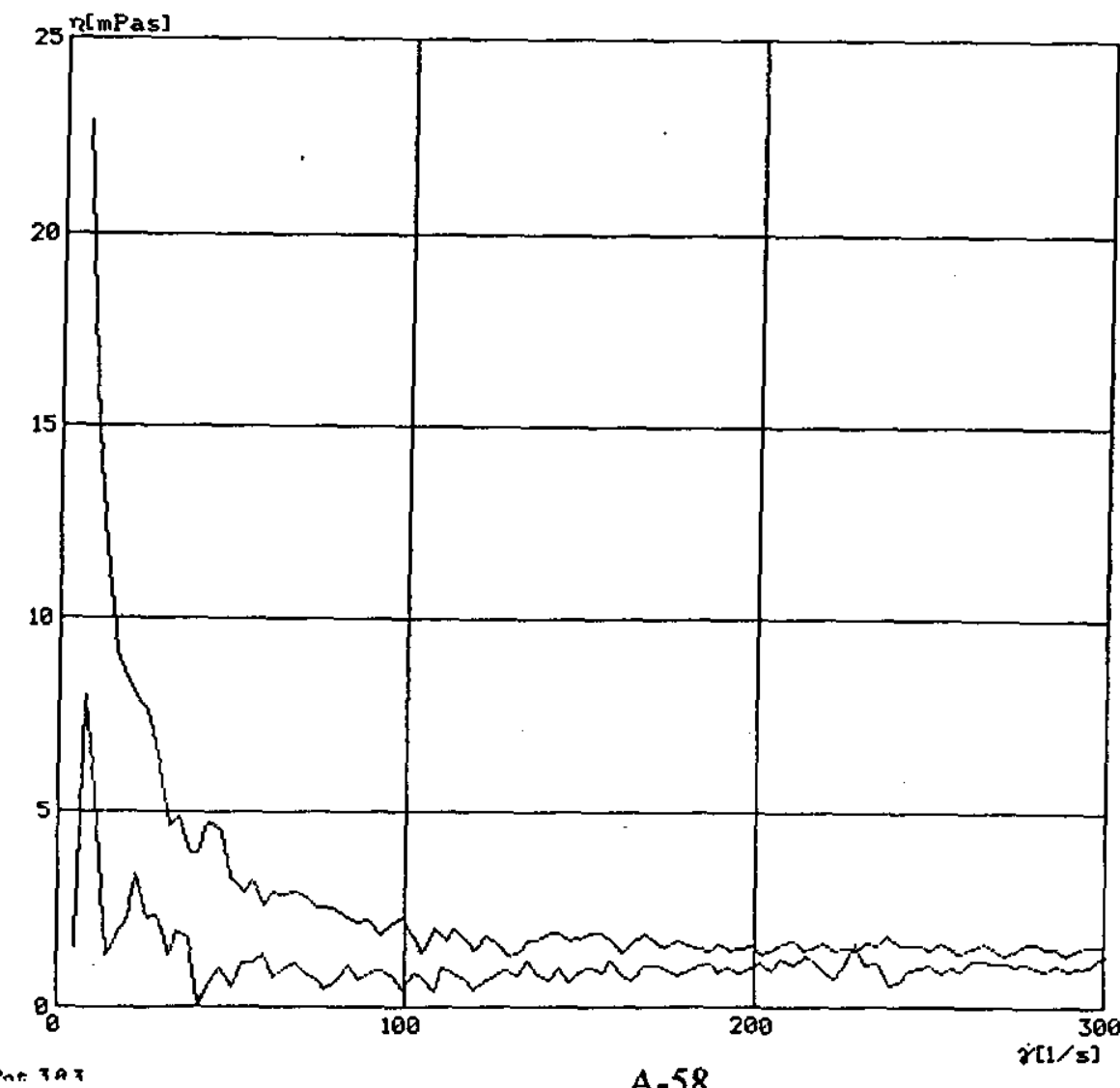

H F F K E

Operator:

jwo

Substanoe:

C104 I- $65 \mathrm{C}$

Test No:

01

Test of:

88-26-1999

System:

M5/MY1

Temperature:

$23.0^{\circ} \mathrm{C}$ 
RPP-5798, Rev. 0

\begin{tabular}{|c|c|c|c|c|}
\hline$\tau[\mathbf{P a}]$ & $\gamma\left[\mathbf{s}^{-1}\right]$ & $\eta[m P a s]$ & t[min] & Temp| ${ }^{\circ} \mathrm{C}$ \\
\hline 0.000 & 0.051 & 0.000 & 0.00 & 64.7 \\
\hline 1.525 & 0.152 & 99.830 & 0.02 & 64.7 \\
\hline 4.899 & 0.158 & 32.340 & 0.04 & 64.7 \\
\hline 7.282 & 0.167 & 22.890 & 0.06 & 64.7 \\
\hline 10.640 & 0.159 & 14.900 & 0.08 & 64.7 \\
\hline 13.460 & 0.161 & 11.950 & 0.10 & 64.7 \\
\hline 16.930 & 0.155 & 9.156 & 0.12 & 64.7 \\
\hline 19.690 & 0.166 & 8.427 & 0.14 & 64.7 \\
\hline 22.700 & 0.179 & 7.877 & 0.16 & 64.7 \\
\hline 25.540 & 0.196 & 7.656 & 0.18 & 64.7 \\
\hline 28.990 & 0.179 & 6.181 & 0.20 & 64.7 \\
\hline 31.890 & 0.148 & 4.643 & 0.22 & 64.7 \\
\hline 34.940 & 0.169 & 4.834 & 0.24 & 64.7 \\
\hline 38.390 & 0.151 & 3.945 & 0.26 & 64.7 \\
\hline 40.560 & 0.160 & 3.941 & 0.27 & 64.7 \\
\hline 43.560 & 0.207 & 4.752 & 0.30 & 64.7 \\
\hline 47.390 & 0.213 & 4.496 & 0.32 & 64.7 \\
\hline 49.770 & 0.164 & 3.288 & 0.34 & 64.7 \\
\hline 53.930 & 0.157 & 2.905 & 0.36 & 64.7 \\
\hline 55.890 & 0.180 & 3.222 & 0.38 & 64.7 \\
\hline 59.090 & 0.154 & 2.598 & 0.40 & 64.7 \\
\hline 62.060 & 0.180 & 2.894 & 0.42 & 64.7 \\
\hline 65.290 & 0.186 & 2.849 & 0.44 & 64.7 \\
\hline 68.150 & 0.200 & 2.931 & 0.46 & 64.7 \\
\hline 71.650 & 0.200 & 2.792 & 0.48 & 64.7 \\
\hline 74.490 & 0.187 & 2.510 & 0.50 & 64.7 \\
\hline 78.210 & 0.200 & 2.557 & 0.52 & 64.7 \\
\hline 79.700 & 0.200 & 2.507 & 0.53 & 64.7 \\
\hline 83.130 & 0.191 & 2.291 & 0.56 & 64.7 \\
\hline 86.560 & 0.188 & 2.171 & 0.58 & 64.7 \\
\hline 89.460 & 0.200 & 2.232 & 0.60 & 64.7 \\
\hline 92.960 & 0.169 & 1.821 & 0.62 & 64.7 \\
\hline 95.690 & 0.203 & 2.118 & 0.64 & 64.7 \\
\hline 99.480 & 0.224 & 2.251 & 0.67 & 64.7 \\
\hline 102.300 & 0.186 & 1.819 & 0.68 & 64.7 \\
\hline 105.100 & 0.145 & 1.379 & 0.71 & 64.7 \\
\hline 108.000 & 0.220 & 2.038 & 0.73 & 64.7 \\
\hline 112.100 & 0.191 & 1.704 & 0.75 & 64.7 \\
\hline 113.800 & 0.232 & 2.039 & 0.77 & 64.7 \\
\hline 117.200 & 0.203 & 1.728 & 0.79 & 64.7 \\
\hline 119.500 & 0.176 & 1.469 & 0.81 & 64.7 \\
\hline 122.900 & 0.227 & 1.848 & 0.82 & 64.7 \\
\hline 126.000 & 0.211 & 1.672 & 0.85 & 64.7 \\
\hline 129.200 & 0.174 & 1.349 & 0.86 & 64.7 \\
\hline 132.100 & 0.187 & 1.415 & 0.89 & 64.7 \\
\hline 135.000 & 0.229 & 1.698 & 0.91 & 64.7 \\
\hline 137.800 & 0.239 & 1.735 & 0.93 & 64.7 \\
\hline 141.200 & 0.269 & 1.903 & 0.95 & 64.7 \\
\hline 144.100 & 0.279 & 1.934 & 0.97 & 64.7 \\
\hline 147.000 & 0.254 & 1.730 & 0.99 & 64.7 \\
\hline 149.800 & 0.271 & 1.807 & 1.01 & 64.7 \\
\hline 153.300 & 0.289 & 1.887 & 1.03 & 64.7 \\
\hline 156.100 & 0.298 & 1.907 & 1.05 & 64.7 \\
\hline 159.200 & 0.262 & 1.648 & 1.07 & 64.7 \\
\hline 161.800 & 0.227 & 1.404 & 1.09 & 64.7 \\
\hline 165.300 & 0.271 & 1.641 & 1.11 & 64.7 \\
\hline 168.100 & 0.321 & 1.908 & 1.13 & 64.7 \\
\hline 171.600 & 0.299 & 1.743 & 1.15 & 64.7 \\
\hline 173.800 & 0.270 & 1.555 & 1.17 & 64.7 \\
\hline 177.300 & 0.299 & 1.687 & 1.19 & 64.7 \\
\hline 180.700 & 0.285 & 1.577 . & 1.21 & 64.7 \\
\hline 183.600 & 0.283 & $1.542^{\circ}$ & 1.23 & 64.7 \\
\hline 187.000 & 0.278 & 1.488 & 1.25 & 64.7 \\
\hline 189.300 & 0.317 & 1.675 & 1.27 & 64.7 \\
\hline 192.700 & 0.286 & 1.483 & 1.29 & 64.7 \\
\hline 195.600 & 0.293 & 1.500 & 1.31 & 64.7 \\
\hline
\end{tabular}


RPP-5798, Rev. 0

\begin{tabular}{|c|c|c|c|c|}
\hline 199.000 & 0.319 & 1.603 & 1.33 & 64.7 \\
\hline 201.500 & 0.287 & 1.426 & 1.35 & 64.7 \\
\hline 204.700 & 0.298 & 1.454 & 1.37 & 64.7 \\
\hline 207.600 & 0.342 & 1.646 & 1.39 & 64.7 \\
\hline 211.000 & 0.359 & 1.699 & 1.41 & 64.7 \\
\hline 213.900 & 0.318 & 1.489 & 1.43 & 64.7 \\
\hline 216.700 & 0.335 & 1.543 & 1.45 & 64.7 \\
\hline 219.600 & 0.357 & 1.624 & 1.47 & 64.7 \\
\hline 223.000 & 0.326 & 1.460 & 1.49 & 64.7 \\
\hline 225.900 & 0.340 & 1.506 & 1.51 & 64.7 \\
\hline 229.100 & 0.342 & 1.491 & 1.53 & 64.7 \\
\hline 231.600 & 0.377 & 1.626 & 1.55 & 64.7 \\
\hline 235.000 & 0.380 & 1.618 & 1.58 & 64.7 \\
\hline 237.900 & 0.440 & 1.851 & 1.59 & 64.7 \\
\hline 241.300 & 0.383 & 1.588 & 1.62 & 64.7 \\
\hline 243.600 & 0.392 & 1.608 & 1.63 & 64.7 \\
\hline 247.000 & 0.396 & 1.605 & 1.66 & 64.7 \\
\hline 249.900 & 0.366 & 1.465 & 1.67 & 64.7 \\
\hline 253.300 & 0.422 & 1.667 & 1.70 & 64.7 \\
\hline 256.200 & 0.376 & 1.466 & 1.71 & 64.7 \\
\hline 259.000 & 0.362 & 1.399 & 1.74 & 64.7 \\
\hline 261.900 & 0.394 & 1.505 & 1.75 & 64.7 \\
\hline 265.300 & 0.436 & 1.642 & 1.78 & 64.7 \\
\hline 268.800 & 0.390 & 1.450 & 1.80 & 64.7 \\
\hline 271.200 & 0.370 & 1.366 & 1.82 & 64.7 \\
\hline 274.500 & 0.403 & 1.467 & 1.84 & 64.7 \\
\hline 277.300 & 0.461 & 1.663 & 1.86 & 64.7 \\
\hline 280.800 & 0.457 & 1.627 & 1.88 & 64.7 \\
\hline 283.600 & 0.430 & 1.514 & 1.90 & 64.7 \\
\hline 286.500 & 0.439 & 1.534 & 1.92 & 64.7 \\
\hline 289.400 & 0.386 & 1.332 & 1.94 & 64.7 \\
\hline 292.800 & 0.447 & 1.527 & 1.96 & 64.7 \\
\hline 295.600 & 0.477 & 1.612 & 1.98 & 64.7 \\
\hline 298.900 & 0.467 & 1.562 & 2.00 & 64.7 \\
\hline 300.200 & 0.397 & 1.324 & 2.00 & 64.7 \\
\hline 298.500 & 0.373 & 1.249 & 2.02 & 64.7 \\
\hline 296.000 & 0.322 & 1.087 & 2.04 & 64.7 \\
\hline 292.600 & 0.304 & 1.040 & 2.06 & 64.7 \\
\hline 289.900 & 0.280 & 0.965 & 2.08 & 64.7 \\
\hline 286.300 & 0.304 & 1.061 & 2.10 & 64.7 \\
\hline 283.800 & 0.277 & 0.976 & 2.12 & 64.7 \\
\hline 280.800 & 0.293 & 1.045 & 2.14 & 64.7 \\
\hline 277.700 & 0.326 & 1.174 & 2.16 & 64.7 \\
\hline 274.300 & 0.298 & 1.087 & 2.18 & 64.7 \\
\hline 271.600 & 0.310 & 1.141 & 2.20 & 64.7 \\
\hline 268.600 & 0.301 & 1.122 & 2.22 & 64.7 \\
\hline 265.700 & 0.316 & 1.188 & 2.24 & 64.7 \\
\hline 262.300 & 0.310 & 1.183 & 2.26 & 64.7 \\
\hline 259.400 & 0.245 & 0.945 & 2.28 & 64.7 \\
\hline 256.400 & 0.264 & 1.028 & 2.30 & 64.7 \\
\hline 253.300 & 0.221 & 0.872 & 2.32 & 64.7 \\
\hline 250.500 & 0.274 & 1.092 & 2.34 & 64.7 \\
\hline 247.200 & 0.249 & 1.007 & 2.36 & 64.7 \\
\hline 244.000 & 0.234 & 0.959 & 2.38 & 64.7 \\
\hline 241.300 & 0.154 & 0.638 & 2.40 & 64.7 \\
\hline 238.500 & 0.133 & 0.559 & 2.42 & 64.7 \\
\hline 235.000 & 0.272 & 1.158 & 2.44 & 64.7 \\
\hline 231.600 & 0.255 & 1.102 & 2.46 & 64.7 \\
\hline 228.700 & 0.373 & 1.630 & 2.48 & 64.7 \\
\hline 226.300 & 0.272 & 1.201 & 2.50 & 64.7 \\
\hline 223.000 & 0.177 & 0.794 & 2.53 & 64.7 \\
\hline 220.200 & 0.198 & 0.901 & 2.54 & 64.7 \\
\hline 216.200 & 0.271 & 1.255 & 2.57 & 64.7 \\
\hline 214.400 & 0.283 & 1.318 & 2.58 & 64.7 \\
\hline 210.800 & 0.223 & 1.056 & 2.61 & 64.7 \\
\hline 207.600 & 0.265 & 1.277 & 2.62 & 64.7 \\
\hline 204.900 & 0.198 & 0.964 & 2.64 & 64.7 \\
\hline 201.900 & 0.235 & 1.163 & 2.66 & 64.7 \\
\hline 199.000 & 0.200 & 1.007 & 2.69 & 64.7 \\
\hline 196.000 & 0.171 & 0.870 & 2.70 & 64.7 \\
\hline
\end{tabular}


RPP-5798, Rev. 0

\begin{tabular}{|c|c|c|c|c|}
\hline $\begin{array}{l}192.300 \\
189.700\end{array}$ & $\begin{array}{l}0.200 \\
0.176\end{array}$ & $\begin{array}{l}1.039 \\
0.925\end{array}$ & $\begin{array}{l}2.73 \\
2.74\end{array}$ & $\begin{array}{l}64.7 \\
64.7\end{array}$ \\
\hline 187.000 & 0.210 & 1.123 & 2.76 & 64.7 \\
\hline 183.800 & 0.202 & 1.098 & 2.79 & 64.7 \\
\hline 180.500 & 0.166 & 0.921 & 2.81 & 64.7 \\
\hline 177.500 & 0.146 & 0.820 & 2.83 & 64.7 \\
\hline 174.000 & 0.181 & 1.041 & 2.85 & 64.7 \\
\hline 171.700 & 0.190 & 1.105 & 2.87 & 64.7 \\
\hline 168.300 & 0.185 & 1.097 & 2.89 & 64.7 \\
\hline 165.100 & 0.118 & 0.714 & 2.91 & 64.7 \\
\hline 162.200 & 0.136 & 0.840 & 2.93 & 64.7 \\
\hline 159.000 & 0.189 & 1.191 & 2.95 & 64.7 \\
\hline 156.300 & 0.143 & 0.915 & 2.97 & 64.7 \\
\hline 152.900 & 0.155 & 1.010 & 2.99 & 64.7 \\
\hline 150.000 & 0.143 & 0.954 & 3.01 & 64.7 \\
\hline 146.600 & 0.099 & 0.674 & 3.03 & 64.7 \\
\hline 144.100 & 0.143 & 0.994 & 3.05 & 64.7 \\
\hline 140.900 & 0.096 & 0.682 & 3.07 & 64.7 \\
\hline 138.000 & 0.113 & 0.816 & 3.09 & 64.7 \\
\hline 134.600 & 0.160 & 1.191 & 3.11 & 64.7 \\
\hline 132.100 & 0.113 & 0.854 & 3.13 & 64.7 \\
\hline 128.900 & 0.120 & 0.931 & 3.15 & 64.7 \\
\hline 126.000 & 0.102 & 0.809 & 3.17 & 64.7 \\
\hline 122.600 & 0.081 & 0.660 & 3.19 & 64.7 \\
\hline 119.700 & 0.053 & 0.442 & 3.21 & 64.7 \\
\hline 116.700 & 0.087 & 0.743 & 3.23 & 64.7 \\
\hline 113.800 & 0.099 & 0.874 & 3.25 & 64.7 \\
\hline 110.400 & 0.115 & 1.043 & 3.27 & 64.7 \\
\hline 108.400 & 0.042 & 0.388 & 3.29 & 64.7 \\
\hline 104.900 & 0.081 & 0.777 & 3.31 & 64.7 \\
\hline 102.400 & 0.093 & 0.912 & 3.33 & 64.7 \\
\hline 98.950 & 0.045 & 0.458 & 3.35 & 64.7 \\
\hline 96.090 & 0.079 & 0.821 & 3.37 & 64.7 \\
\hline 92.620 & 0.088 & 0.952 & 3.39 & 64.7 \\
\hline 90.160 & 0.080 & 0.884 & 3.41 & 64.7 \\
\hline 86.840 & 0.059 & 0.676 & 3.43 & 64.7 \\
\hline 83.970 & 0.089 & 1.056 & 3.45 & 64.7 \\
\hline 80.500 & 0.057 & 0.706 & 3.47 & 64.7 \\
\hline 77.010 & 0.035 & 0.449 & 3.50 & 64.7 \\
\hline 74.660 & 0.053 & 0.713 & 3.51 & 64.6 \\
\hline 71.270 & 0.065 & 0.916 & 3.54 & 64.7 \\
\hline 68.370 & 0.078 & 1.140 & 3.55 & 64.7 \\
\hline 64.940 & 0.064 & 0.990 & 3.58 & 64.7 \\
\hline 62.350 & 0.047 & 0.756 & 3.59 & 64.7 \\
\hline 59.170 & 0.084 & 1.413 & 3.62 & 64.7 \\
\hline 56.310 & 0.065 & 1.148 & 3.63 & 64.6 \\
\hline 52.880 & 0.062 & 1.179 & 3.66 & 64.7 \\
\hline 49.940 & 0.025 & 0.510 & 3.67 & 64.6 \\
\hline 46.990 & 0.049 & 1.033 & 3.70 & 64.7 \\
\hline 44.200 & 0.030 & 0.669 & 3.72 & 64.7 \\
\hline 40.720 & 0.004 & 0.106 & 3.74 & 64.7 \\
\hline 37.880 & 0.067 & 1.780 & 3.76 & 64.7 \\
\hline 34.540 & 0.068 & 1.977 & 3.78 & 64.7 \\
\hline 32.080 & 0.044 & 1.359 & 3.80 & 64.7 \\
\hline 28.670 & 0.068 & 2.362 & 3.82 & 64.7 \\
\hline 25.810 & 0.059 & 2.288 & 3.84 & 64.7 \\
\hline 22.360 & 0.077 & 3.442 & 3.86 & 64.7 \\
\hline 19.880 & 0.045 & 2.255 & 3.88 & 64.7 \\
\hline 16.620 & 0.030 & 1.814 & 3.90 & 64.7 \\
\hline 13.720 & 0.019 & 1.357 & 3.92 & 64.7 \\
\hline 10.310 & 0.056 & 5.457 . & 3.94 & 64.7 \\
\hline 7.434 & 0.060 & 8.040 & 3.96 & 64.7 \\
\hline 4.499 & 0.007 & 1.503 & 3.98 & 64.7 \\
\hline 1.677 & 0.044 & 26.280 & 4.00 & 64.7 \\
\hline
\end{tabular}


RPP-5798, Rev. 0

VISCOȘITY ANALYSIS OF

DILUTION LEVEL "+" (140 g solids/L)

AT AMBIENT TEMPERATURE

A-62 


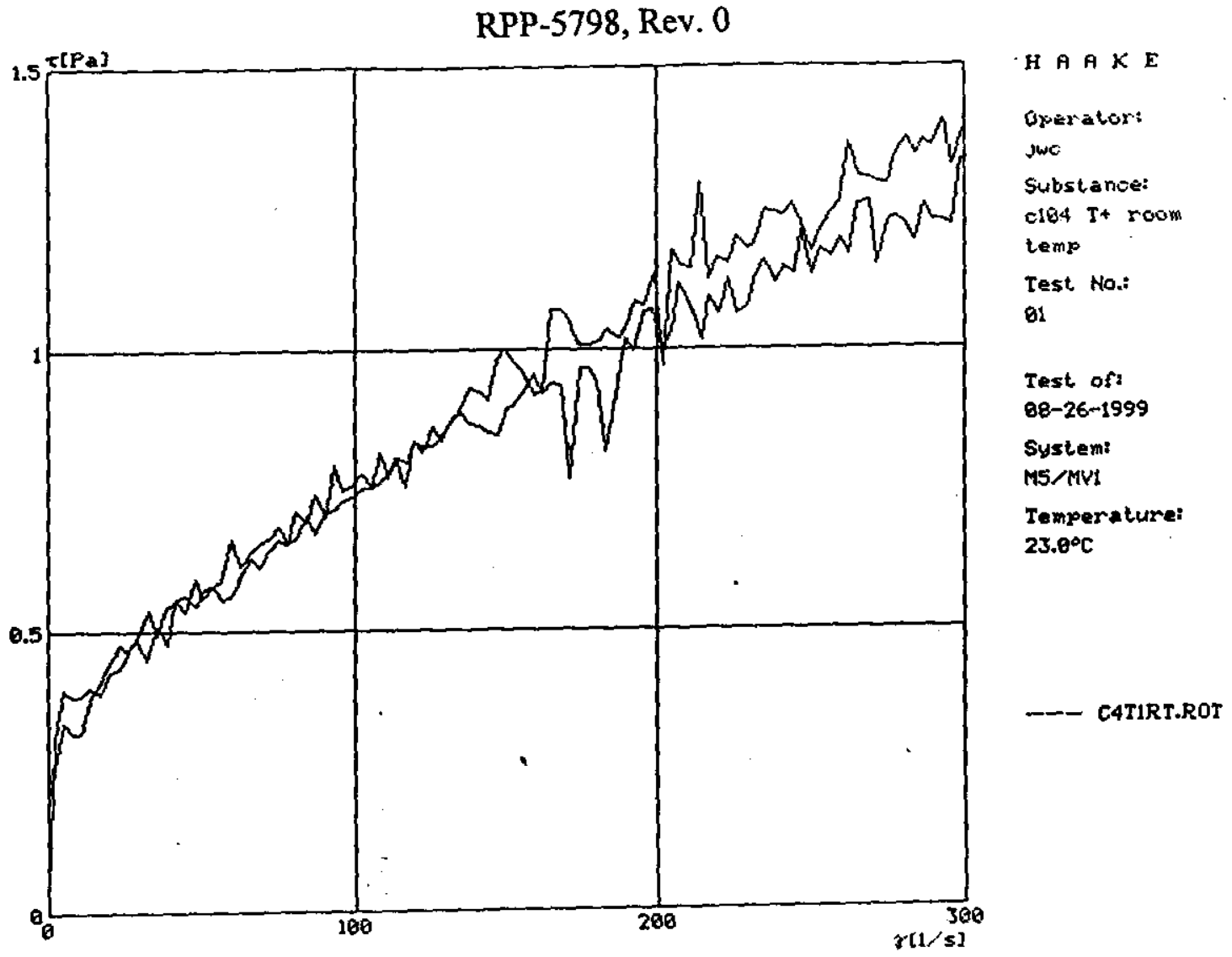

WAAKF PAK 3 ด 3

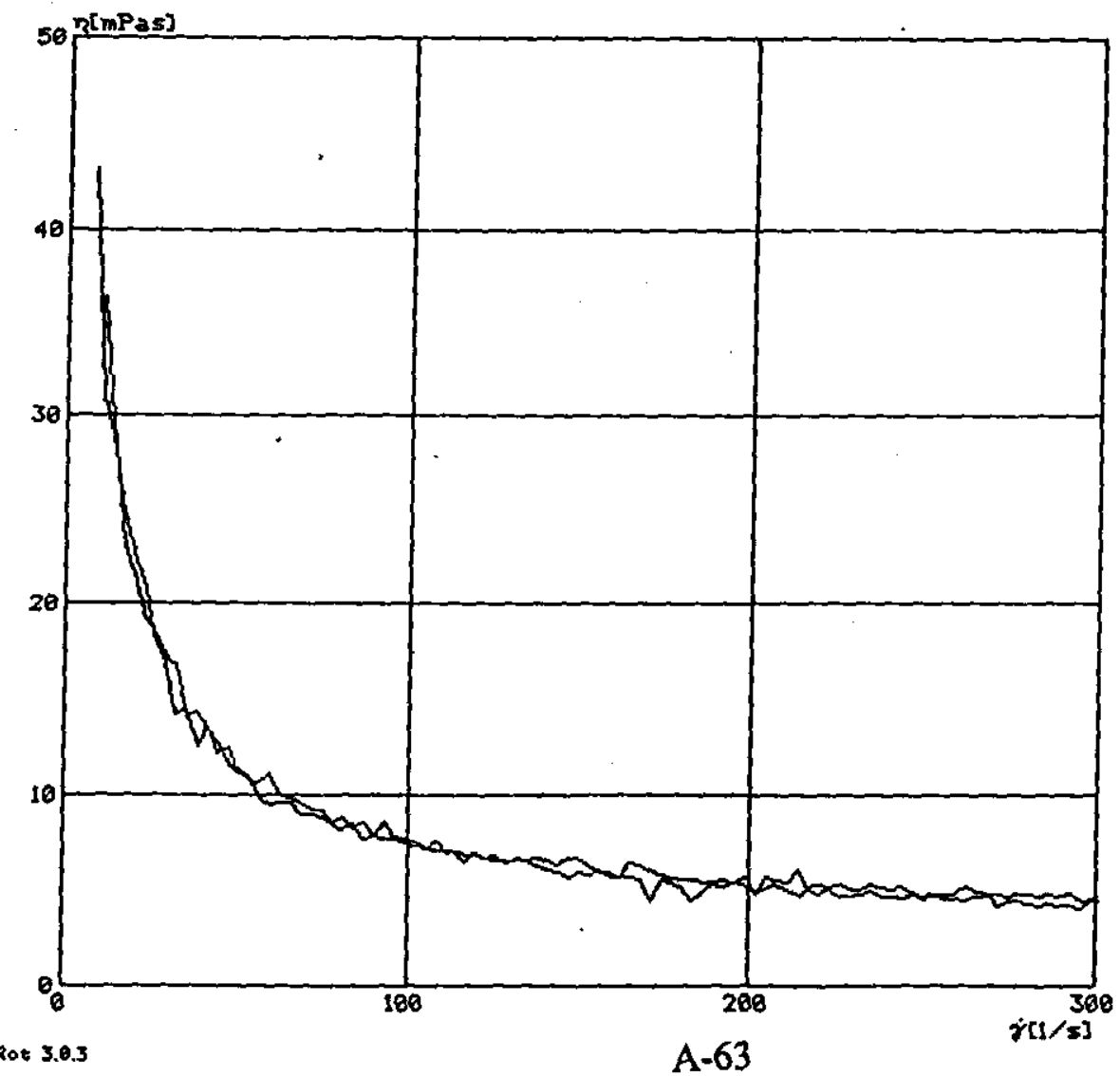

I $A$ ค $\mathrm{K}$

Operator:

two

Substance: c104 I+ room

teme

Test Ho:

a)

Test of: 88-26-1999

System:

MS/MV1

Temperature:

$23.0^{\circ} \mathrm{C}$ 
RPP-5798, Rev. 0

\begin{tabular}{|c|c|c|c|c|}
\hline$\tau[\mathbf{P a}]$ & $\gamma\left|s^{-1}\right|$ & $\eta[\mathbf{m P s s}]$ & t]min] & Temp $\mid{ }^{\circ} \mathrm{C}$ \\
\hline 0.000 & 0.034 & 0.000 & 0.00 & 26.2 \\
\hline 1.944 & 0.315 & 161.900 & 0.02 & 26.2 \\
\hline 4.880 & 0.397 & 81.340 & 0.04 & 26.3 \\
\hline 7.339 & 0.388 & 52.800 & 0.06 & 26.3 \\
\hline 10.660 & 0.388 & 36.370 & 0.08 & 26.2 \\
\hline 13.550 & 0.401 & 29.600 & 0.10 & 26.3 \\
\hline 16.960 & 0.391 & 23.060 & 0.12 & 26.3 \\
\hline 19.840 & 0.428 & 21.550 & 0.14 & 26.3 \\
\hline 22.760 & 0.437 & 19.210 & 0.16 & 26.3 \\
\hline 25.600 & 0.470 & 18.370 & 0.18 & 26.3 \\
\hline 28.990 & 0.500 & 17.230 & 0.20 & 26.3 \\
\hline 31.910 & 0.536 & 16.790 & 0.22 & 26.3 \\
\hline 34.960 & 0.491 & 14.040 & 0.24 & 26.3 \\
\hline 37.670 & 0.542 & 14.390 & 0.26 & 26.3 \\
\hline 41.120 & 0.553 & 13.460 & 0.28 & 26.3 \\
\hline 43.990 & 0.532 & 12.090 & 0.30 & 26.3 \\
\hline 47.460 & 0.595 & 12.530 & 0.32 & 26.3 \\
\hline 49.870 & 0.558 & 11.190 & 0.34 & 26.3 \\
\hline 53.240 & 0.580 & 10.900 & 0.36 & 26.3 \\
\hline 56.060 & 0.592 & 10.560 & 0.38 & 26.3 \\
\hline 59.530 & 0.663 & 11.140 & 0.40 & 26.3 \\
\hline 62.160 & 0.619 & 9.951 & 0.42 & 26.3 \\
\hline 65.320 & 0.640 & 9.801 & 0.44 & 26.3 \\
\hline 68.180 & 0.654 & 9.584 & 0.46 & 26.3 \\
\hline 71.690 & 0.666 & 9.287 & 0.48 & 26.3 \\
\hline 74.530 & 0.684 & 9.180 & 0.50 & 26.3 \\
\hline 77.540 & 0.656 & 8,458 & 0.52 & 26.3 \\
\hline 80.290 & 0.713 & 8.881 & 0.54 & 26.3 \\
\hline 83.720 & 0.691 & 8.252 & 0.56 & 26.3 \\
\hline 86.650 & 0.740 & 8.539 & 0.58 & 26.3 \\
\hline 89.990 & 0.705 & 7.829 & 0.60 & 26.3 \\
\hline 93.040 & 0.795 & 8.542 & 0.63 & 26.3 \\
\hline 95.880 & 0.748 & 7.796 & 0.64 & 26.3 \\
\hline 99.330 & 0.762 & 7.673 & 0.67 & 26.3 \\
\hline 102.200 & 0.779 & 7.625 & 0.68 & 26.3 \\
\hline 105.300 & 0.755 & 7.174 & 0.71 & 26.3 \\
\hline 108.000 & 0.816 & 7.551 & 0.73 & 26.3 \\
\hline 111.300 & 0.771 & 6.927 & 0.75 & 26.3 \\
\hline 113.600 & 0.805 & 7.087 & 0.77 & 26.3 \\
\hline 117.200 & 0.798 & 6.808 & 0.79 & 26.3 \\
\hline 119.500 & 0.836 & 6.994 & 0.81 & 26.3 \\
\hline 122.900 & 0.826 & 6.722 & 0.83 & 26.3 \\
\hline 125.800 & 0.828 & 6.580 & 0.85 & 26.3 \\
\hline 129.200 & 0.851 & 6.585 & 0.87 & 26.3 \\
\hline 131.700 & 0.865 & 6.565 & 0.89 & 26.3 \\
\hline 135.000 & 0.894 & 6.624 & 0.91 & 26.3 \\
\hline 137.800 & 0.930 & 6.751 & 0.93 & 26.3 \\
\hline 141.200 & 0.925 & 6.547 & 0.95 & 26.3 \\
\hline 144.100 & 0.910 & 6.312 & 0.97 & 26.3 \\
\hline 147.000 & 0.979 & 6.662 & 0.99 & 26.3 \\
\hline 149.800 & 0.999 & 6.667 & 1.01 & 26.3 \\
\hline 153.300 & 0.972 & 6.345 & 1.03 & 26.4 \\
\hline 156.100 & 0.958 & 6.138 & 1.05 & 26.4 \\
\hline 159.500 & 0.916 & 5.742 & 1.07 & 26.4 \\
\hline 161.800 & 0.923 & 5.705 & 1.09 & 26.3 \\
\hline 165.300 & 1.067 & 6.456 & 1.11 & 26.4 \\
\hline 168.100 & 1.065 & 6.335 & 1.13 & 26.3 \\
\hline 171.600 & 1.049 & 6.114 & 1.15 & 26.4 \\
\hline 173.800 & 1.006 & 5.787 & 1.17 & 26.4 \\
\hline 177.300 & 1.007 & 5.683 & 1.19 & 26.4 \\
\hline 180.700 & 1.013 & 5.606 & 1.21 & 26.4 \\
\hline 183.600 & 1.033 & 5.629 & 1.23 & 26.4 \\
\hline 187.000 & 1.018 & 5.443 & 1.25 & 26.3 \\
\hline 189.300 & 1.034 & 5.464 & 1.27 & 26.4 \\
\hline 192.700 & 1.082 & 5.615 & 1.29 & 26.3 \\
\hline 195.600 & 1.073 & 5.487 & 1.31 & 26.3 \\
\hline
\end{tabular}


RPP-5798, Rev. 0

\begin{tabular}{|c|c|c|c|c|}
\hline 199.000 & 1.130 & 5.679 & 1.33 & 26.4 \\
\hline 201.700 & 0.970 & 4.810 & 1.35 & 26.3 \\
\hline 204.700 & 1.172 & 5.724 & 1.37 & 26.4 \\
\hline 207.600 & 1.144 & 5.511 & 1.39 & 26.3 \\
\hline 211.000 & 1.137 & 5.390 & 1.41 & 26.3 \\
\hline 213.900 & 1.290 & 6.033 & 1.43 & 26.4 \\
\hline 216.700 & 1.120 & 5.169 & 1.45 & 26.4 \\
\hline 219.600 & 1.159 & 5.278 & 1.47 & 26.4 \\
\hline 223.000 & 1.148 & 5.146 & 1.49 & 26.4 \\
\hline 225.900 & 1.194 & 5.284 & 1.51 & 26.4 \\
\hline 229.100 & 1.173 & 5.118 & 1.53 & 26.4 \\
\hline 231.600 & 1.180 & 5.094 & 1.55 & 26.4 \\
\hline 235.000 & 1.240 & 5.277 & 1.58 & 26.4 \\
\hline 237.900 & 1.237 & 5.198 & 1.59 & 26.4 \\
\hline 241.300 & 1.232 & 5.104 & 1.62 & 26.4 \\
\hline 243.600 & 1.251 & 5.134 & 1.63 & 26.4 \\
\hline 247.000 & 1.216 & 4.923 & 1.66 & 26.4 \\
\hline 249.900 & 1.168 & 4.674 & 1.67 & 26.4 \\
\hline 253.300 & 1.207 & 4.765 & 1.70 & 26.4 \\
\hline 256.200 & 1.238 & 4.832 & 1.71 & 26.4 \\
\hline 259.000 & 1.257 & 4.852 & 1.74 & 26.4 \\
\hline 261.900 & 1.362 & 5.199 & 1.75 & 26.4 \\
\hline 265.300 & 1.302 & 4.908 & 1.78 & 26.4 \\
\hline 268.800 & 1.299 & 4.833 & 1.80 & 26.4 \\
\hline 271.200 & 1.290 & 4.755 & 1.82 & 26.4 \\
\hline 274.500 & 1.287 & 4.689 & 1.84 & 26.4 \\
\hline 277.300 & 1.342 & 4.840 & 1.86 & 26.4 \\
\hline 280.800 & 1.370 & 4.880 & 1.88 & 26.4 \\
\hline 283.600 & 1.339 & 4.719 & 1.90 & 26.4 \\
\hline 286.500 & 1.367 & 4.771 & 1.92 & 26.4 \\
\hline 289.400 & 1.354 & 4.678 & 1.94 & 26.4 \\
\hline 292.800 & 1.402 & 4.790 & 1.96 & 26.4 \\
\hline 295.600 & 1.321 & 4.468 & 1.98 & 26.4 \\
\hline 298.900 & 1.380 & 4.616 & 2.00 & 26.4 \\
\hline 300.200 & 1.330 & 4.429 & 2.00 & 26.5 \\
\hline 298.500 & 1.327 & 4.446 & 2.02 & 26.5 \\
\hline 295.500 & 1.214 & 4.108 & 2.04 & 26.4 \\
\hline 292.600 & 1.222 & 4.178 & 2.06 & 26.4 \\
\hline 289.200 & 1.221 & 4.222 & 2.08 & 26.4 \\
\hline 286.900 & 1.252 & 4.365 & 2.10 & 26.5 \\
\hline 283.400 & 1.185 & 4.182 & 2.12 & 26.5 \\
\hline 280.600 & 1.218 & 4.340 & 2.14 & 26.4 \\
\hline 277.300 & 1.230 & 4.435 & 2.16 & 26.4 \\
\hline 274.500 & 1.218 & 4.436 & 2.18 & 26.5 \\
\hline 271.400 & 1.143 & 4.213 & 2.20 & 26.4 \\
\hline 268.800 & 1.254 & 4.666 & 2.22 & 26.5 \\
\hline 265.100 & 1.253 & 4.724 & 2.24 & 26.5 \\
\hline 262.300 & 1.162 & 4.432 & 2.26 & 26.4 \\
\hline 259.200 & 1.193 & 4.600 & 2.28 & 26.4 \\
\hline 256.600 & 1.158 & 4.514 & 2.30 & 26.5 \\
\hline 253.100 & 1.172 & 4.629 & 2.32 & 26.4 \\
\hline 250.300 & 1.129 & 4.510 & 2.34 & 26.4 \\
\hline 247.000 & 1.202 & 4.864 & 2.36 & 26.5 \\
\hline 244.400 & 1.128 & 4.617 & 2.38 & 26.5 \\
\hline 241.100 & 1.142 & 4.734 & 2.40 & 26.5 \\
\hline 238.100 & 1.113 & 4.674 & 2.42 & 26.4 \\
\hline 234.800 & 1.154 & 4.915 & 2.44 & 26.4 \\
\hline 232.200 & 1.127 & 4.856 & 2.46 & 26.4 \\
\hline 228.900 & 1.069 & 4.669 & 2.48 & 26.4 \\
\hline 226.100 & 1.058 & 4.682 & 2.50 & 26.4 \\
\hline 222.600 & 1.119 & 5.025 & 2.52 & 26.5 \\
\hline 220.000 & 1.058 & 4.809 & 2.54 & 26.5 \\
\hline 216.900 & 1.091 & 5.029 & 2.56 & 26.5 \\
\hline 214.300 & 1.014 & 4.730 & 2.58 & 26.5 \\
\hline 210.800 & 1.071 & 5.081 & 2.60 & 26.5 \\
\hline 207.200 & 1.114 & 5.375 & 2.63 & 26.5 \\
\hline 204.700 & 1.029 & 5.025 & 2.64 & 26.5 \\
\hline 201.500 & 0.997 & 4.948 & 2.67 & 26.5 \\
\hline 198.800 & 1.065 & 5.357 & 2.69 & 26.5 \\
\hline 195.400 & 1.063 & 5.439 & 2.71 & 26.5 \\
\hline
\end{tabular}


RPP-5798, Rev. 0

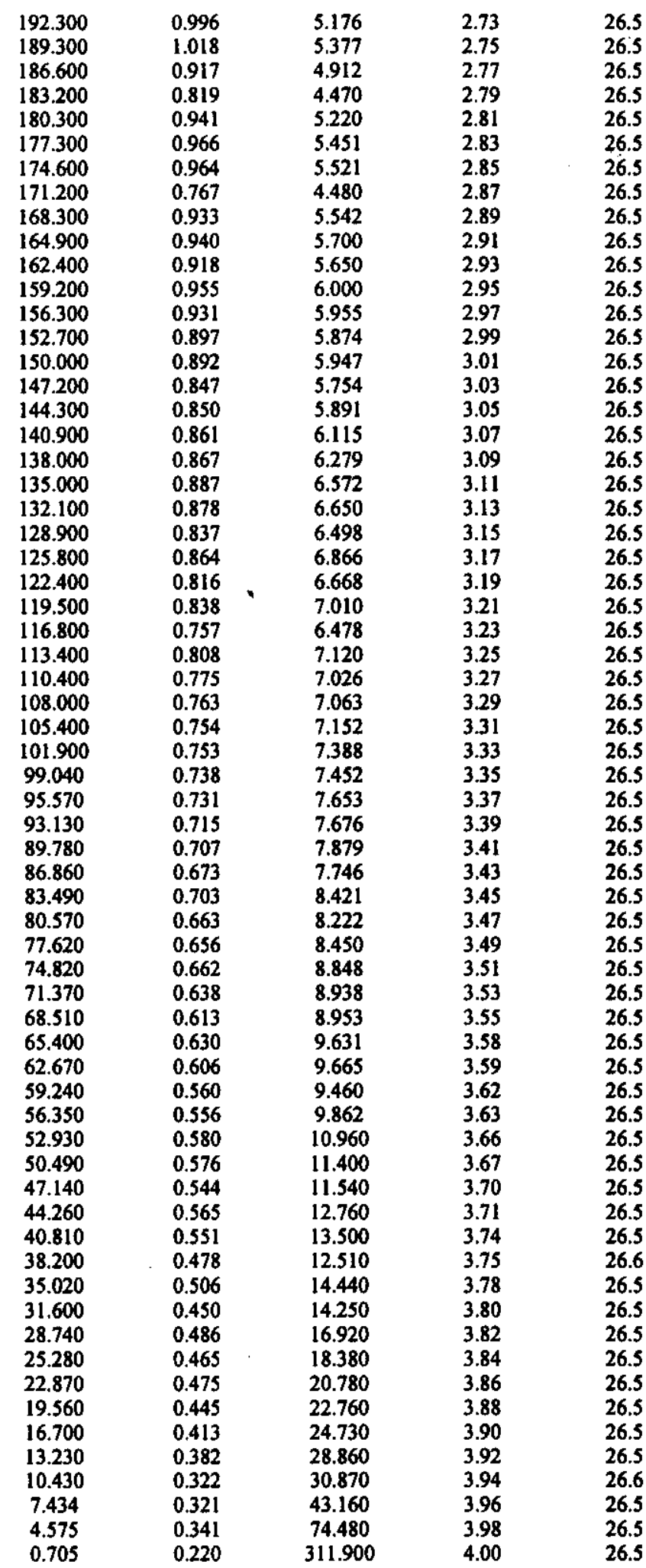


RPP-5798, Rev. 0

\section{VISCOSITY ANALYSIS OF}

DILUTION LEVEL "+" (140 g solids/L) AT $45^{\circ} \mathrm{C}$ 


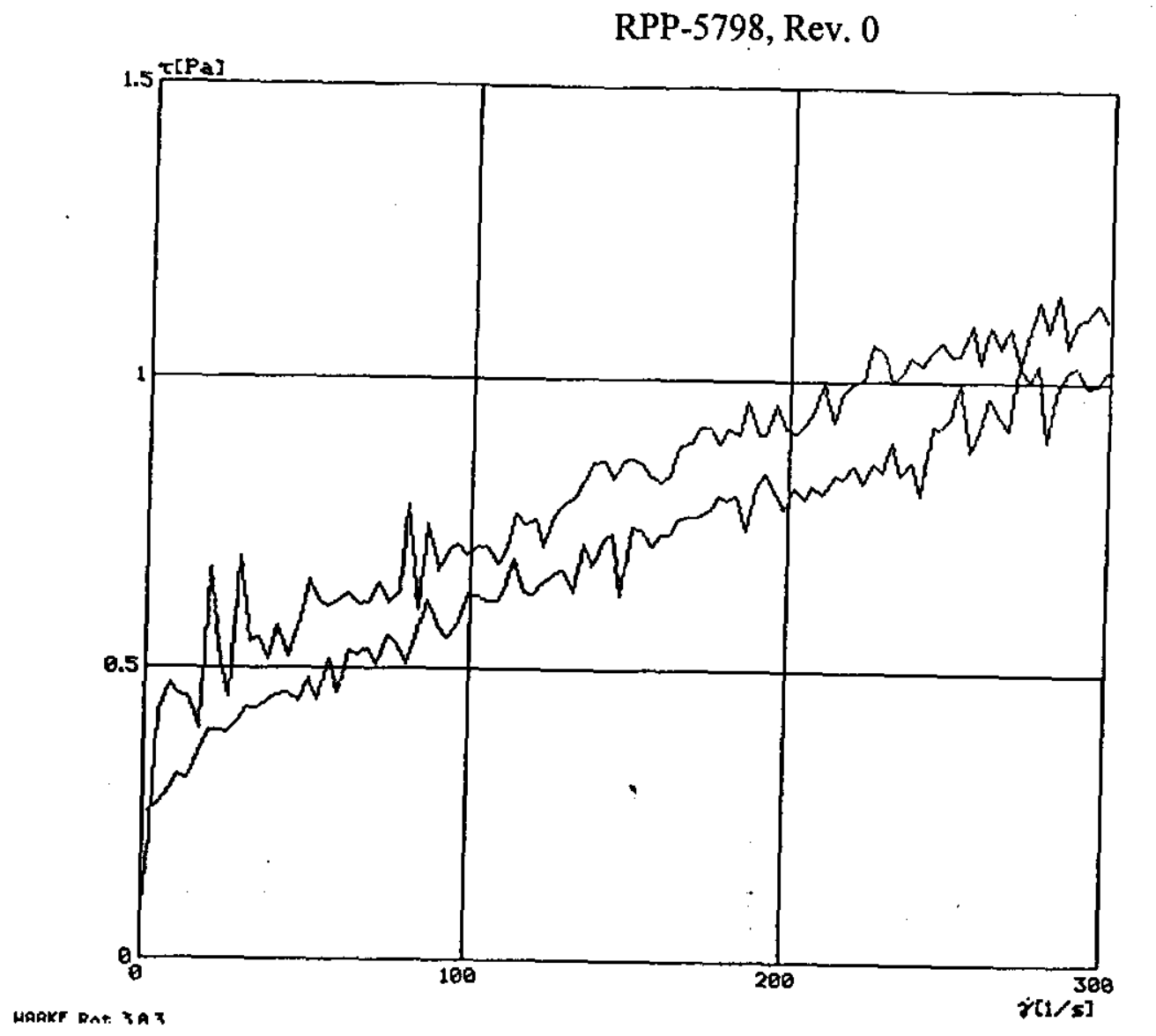

HคA $\mathrm{K} \mathrm{E}$

Operator:

Jwe

Substance:

c104 T+ $45 \mathrm{C}$

Test No.:

o1

Test of:

08-26-1999

System:

MS/MV1

Temperature:

$23.0^{\circ} \mathrm{C}$

C4T145.ROT

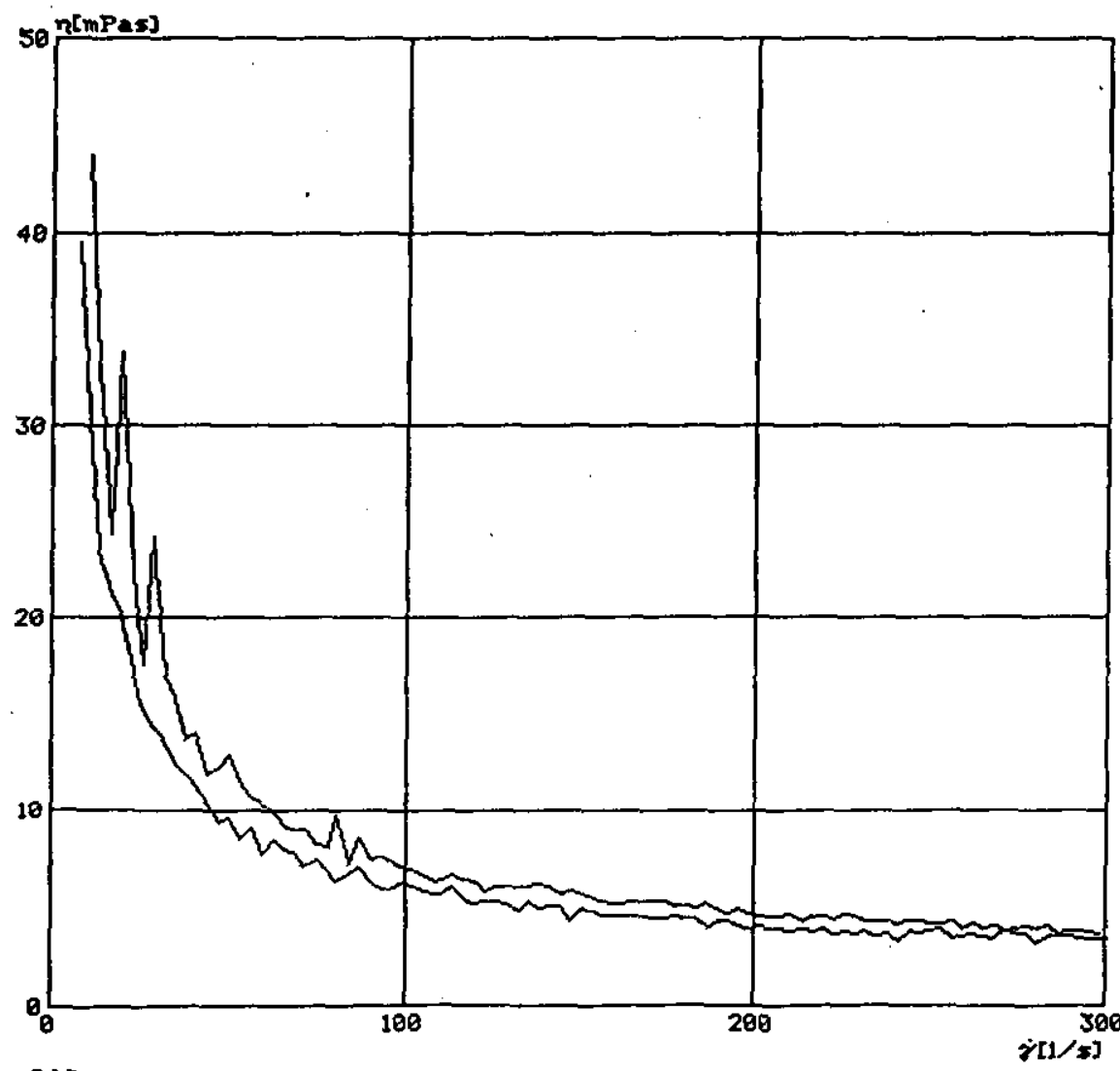

Hค ค $\boldsymbol{K} \mathbf{E}$

Operators

Jwo

Substanos: c104 $\mathrm{T}+45 \mathrm{C}$

Test No.2

01

Test of: 68-26-1999

Systems MS/MV1

Temperature: $23.0^{\circ} \mathrm{C}$

- C4T145.ROT 
RPP-5798, Rev. 0

\begin{tabular}{|c|c|c|c|c|}
\hline$\tau[\mathbf{P a}]$ & $\gamma\left[s^{-1}\right]$ & $\eta[\mathrm{mPas}]$ & $t[m i n]$ & $\operatorname{Temp}^{\circ} \mathrm{Cl}$ \\
\hline 0.000 & 0.082 & 0.000 & 0.00 & 44.9 \\
\hline 1.506 & 0.164 & 109.200 & 0.02 & 44.9 \\
\hline 4.213 & 0.424 & 100.700 & 0.04 & 44.9 \\
\hline 7.796 & 0.473 & 60.700 & 0.06 & 44.9 \\
\hline 10.290 & 0.453 & 44.050 & 0.08 & 44.9 \\
\hline 13.570 & 0.449 & 33.090 & 0.10 & 45.0 \\
\hline 16.390 & 0.400 & 24.370 & 0.12 & 44.9 \\
\hline 19.840 & 0.670 & 33.780 & 0.14 & 44.9 \\
\hline 22.740 & 0.507 & 22.310 & 0.16 & 44.9 \\
\hline 25.680 & 0.449 & 17.490 & 0.18 & 44.9 \\
\hline 28.420 & 0.688 & 24.200 & 0.20 & 44.9 \\
\hline 31.890 & 0.545 & 17.090 & 0.22 & 45.0 \\
\hline 34.810 & 0.551 & 15.840 & 0.24 & 45.0 \\
\hline 37.970 & 0.516 & 13.600 & 0.26 & 45.0 \\
\hline 40.600 & 0.571 & 14.050 & 0.28 & 44.9 \\
\hline 43.980 & 0.520 & 11.830 & 0.30 & 44.9 \\
\hline 47.460 & 0.584 & 12.300 & 0.32 & 44.9 \\
\hline 50.320 & 0.650 & 12.920 & 0.34 & 45.0 \\
\hline 53.300 & 0.616 & 11.560 & 0.36 & 45.0 \\
\hline 56.120 & 0.605 & 10.770 & 0.38 & 45.0 \\
\hline 59.570 & 0.617 & 10.360 & 0.40 & 45.0 \\
\hline 62.410 & 0.630 & 10.090 & 0.42 & 45.0 \\
\hline 65.800 & 0.611 & 9.289 & 0.44 & 45.0 \\
\hline 68.280 & 0.614 & 8.986 & 0.46 & 45.0 \\
\hline 71.710 & 0.648 & 9.037 & 0.48 & 45.0 \\
\hline 74.510 & 0.615 & 8.253 & 0.50 & 45.0 \\
\hline 78.000 & 0.631 & 8.090 & 0.52 & 45.0 \\
\hline 80.520 & 0.782 & 9.715 & 0.54 & 45.0 \\
\hline 83.740 & 0.601 & 7.180 & 0.56 & 45.0 \\
\hline 86.690 & 0.750 & 8.647 & 0.58 & 45.0 \\
\hline 90.090 & 0.670 & 7.435 & 0.61 & 44.9 \\
\hline 93.000 & 0.704 & 7.573 & 0.62 & 44.9 \\
\hline 95.940 & 0.716 & 7.459 & 0.65 & 45.0 \\
\hline 98.740 & 0.697 & 7.056 & 0.66 & 45.0 \\
\hline 102.200 & 0.711 & 6.957 & 0.69 & 44.9 \\
\hline 105.100 & 0.711 & 6.762 & 0.70 & 45.0 \\
\hline 108.200 & 0.682 & 6.304 & 0.73 & 44.9 \\
\hline 110.900 & 0.712 & 6.420 & 0.74 & 44.9 \\
\hline 113.800 & 0.770 & 6.762 & 0.77 & 44.9 \\
\hline 116.700 & 0.750 & 6.430 & 0.78 & 44.9 \\
\hline 120.100 & 0.758 & 6.309 & 0.81 & 44.9 \\
\hline 122.400 & 0.712 & 5.817 & 0.82 & 45.0 \\
\hline 125.800 & 0.766 & 6.086 & 0.85 & 45.0 \\
\hline 128.700 & 0.785 & 6.098 & 0.87 & 44.9 \\
\hline 132.100 & 0.799 & 6.047 & 0.89 & 44.9 \\
\hline 135.000 & 0.825 & 6.115 & 0.91 & 44.9 \\
\hline 137.800 & 0.856 & 6.211 & 0.93 & 44.9 \\
\hline 141.200 & 0.860 & 6.090 & 0.95 & 45.0 \\
\hline 144.100 & 0.830 & 5.756 & 0.97 & 45.0 \\
\hline 147.500 & 0.860 & 5.830 & 0.99 & 44.9 \\
\hline 149.800 & 0.863 & 5.757 & 1.01 & 44.9 \\
\hline 153.300 & 0.855 & 5.576 & 1.03 & 44.9 \\
\hline 156.100 & 0.834 & 5.343 & 1.05 & 44.9 \\
\hline 139.500 & 0.826 & 5.178 & 1.07 & 45.0 \\
\hline 162.400 & 0.835 & 5.139 & 1.09 & 45.0 \\
\hline 165.300 & 0.888 & 5.371 & 1.11 & 45.0 \\
\hline 168.100 & 0.894 & 5.319 & 1.13 & 45.0 \\
\hline 171.600 & 0.920 & 5.361 & 1.15 & 45.0 \\
\hline 174.400 & 0.920 & 5.273 & 1.17 & 44.9 \\
\hline 177.500 & 0.892 & 5.025 & 1.19 & 45.0 \\
\hline 180.100 & 0.920 & 5.108 & 1.21 & 45.0 \\
\hline 183.600 & 0.908 & 4.949 & 1.23 & 44.9 \\
\hline 186.400 & 0.965 & 5.177 & 1.25 & 45.0 \\
\hline 189.900 & 0.907 & 4.780 & 1.27 & 45.0 \\
\hline 192.100 & 0.909 & 4.730 & 1.29 & 45.0 \\
\hline 195.600 & 0.961 & 4.913 & 1.31 & 45.0 \\
\hline
\end{tabular}


RPP-5798, Rev. 0

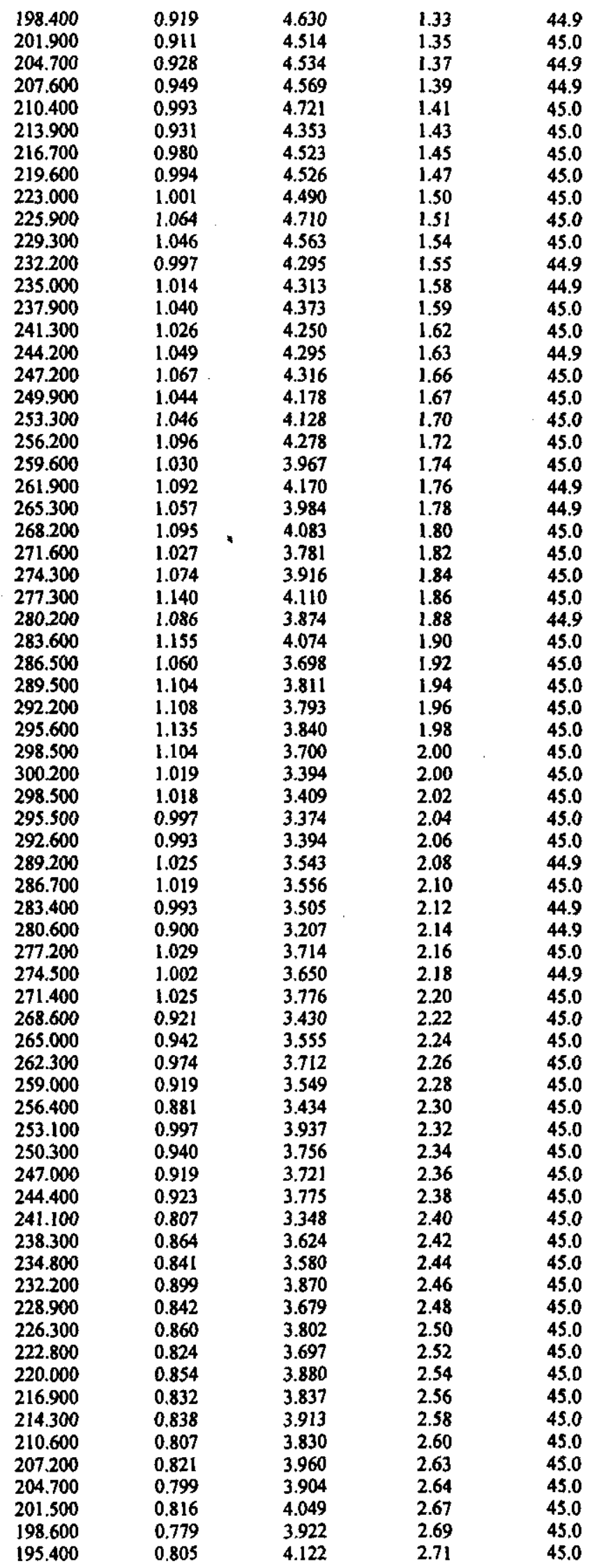


RPP-5798, Rev. 0

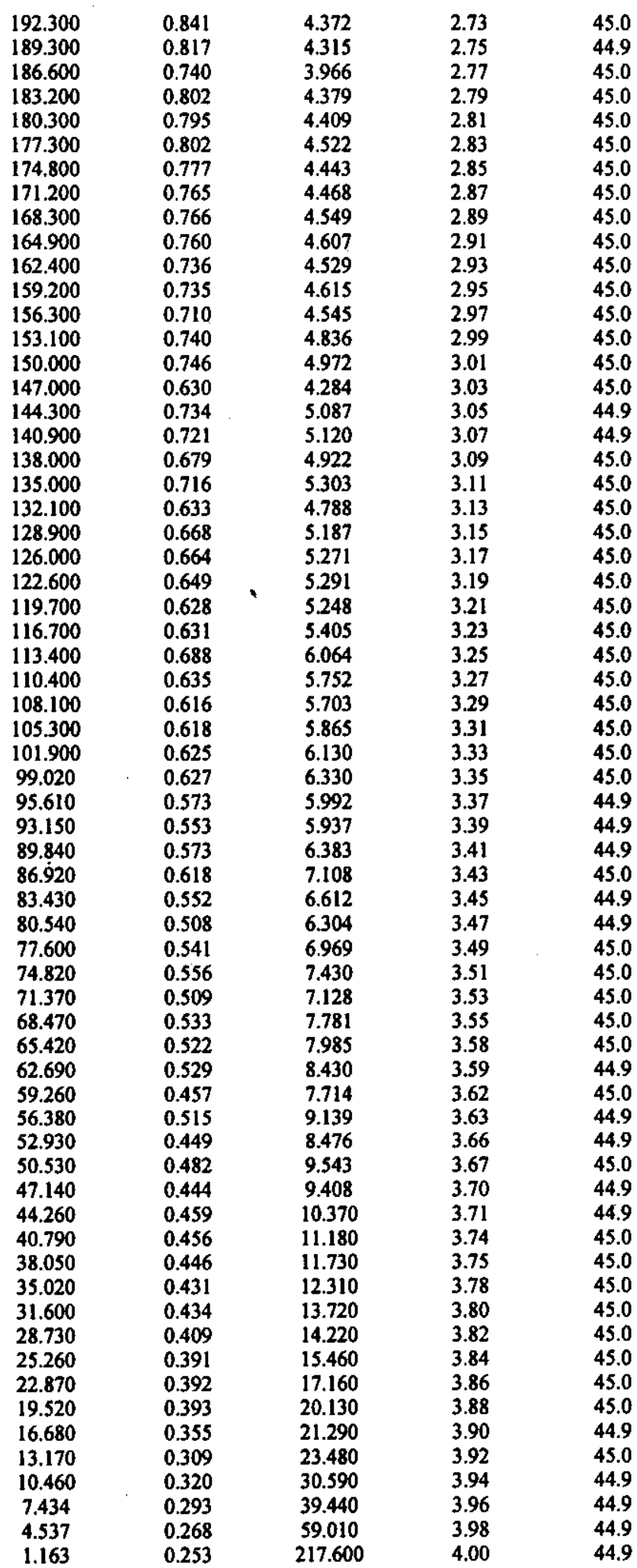


RPP-5798, Rev. 0

\section{VISCOSITY ANALYSIS OF}

\section{DILUTION LEVEL "+" (140 g solids/L) AT $65^{\circ} \mathrm{C}$}


RPP-5798, Rev. 0
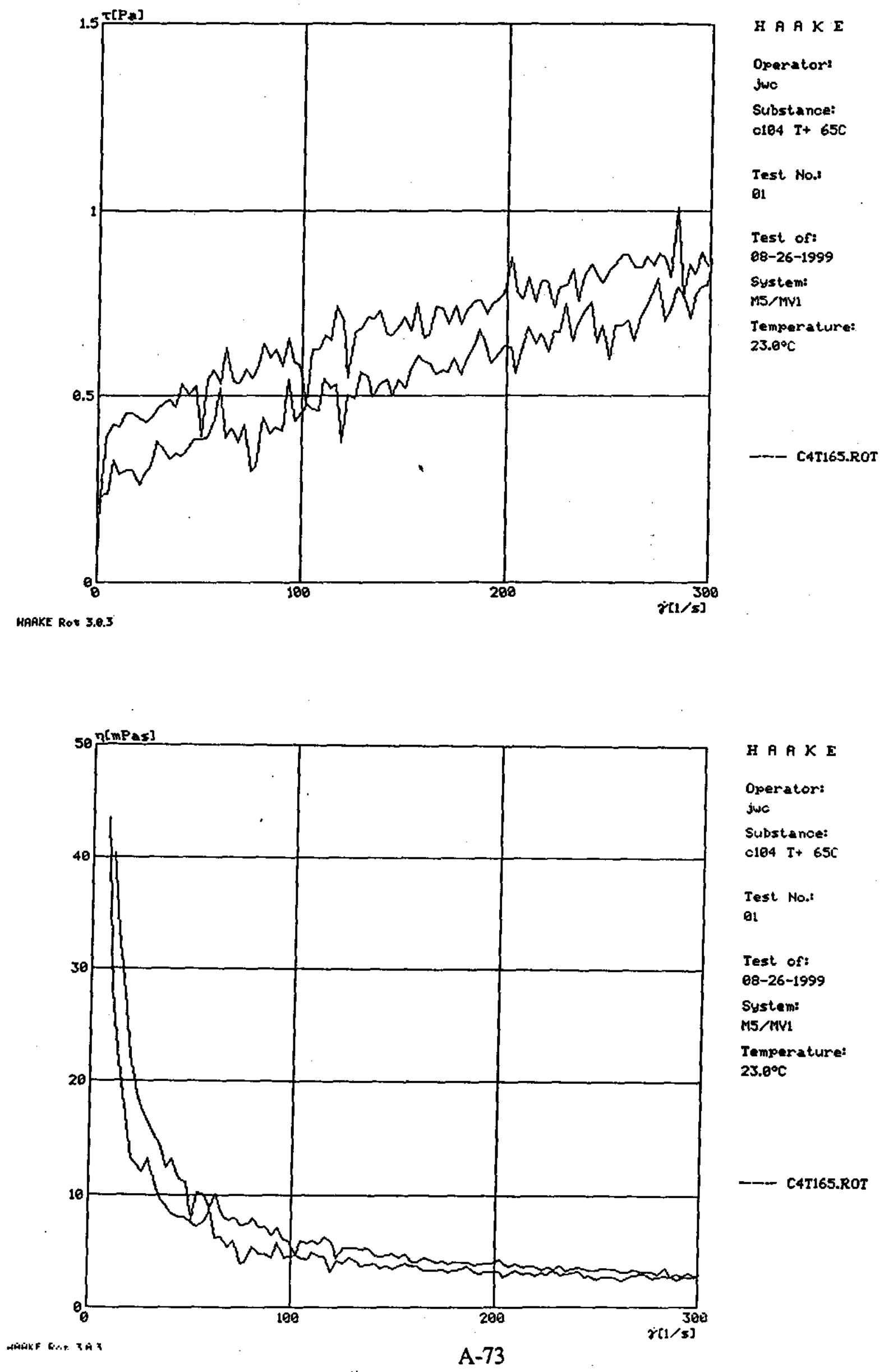
RPP-5798, Rev, 0

\begin{tabular}{|c|c|c|c|c|}
\hline$\tau[\mathbf{P a}]$ & $\gamma\left[\mathbf{s}^{-1}\right]$ & $\eta[m P a s]$ & t[min] & $\left.\operatorname{Temp}\right|^{\circ} \mathrm{Cl}$ \\
\hline 0.000 & 0.055 & 0.000 & 0.00 & 64.3 \\
\hline 1.411 & 0.227 & 160.600 & 0.02 & 64.3 \\
\hline 4.289 & 0.389 & 90.730 & 0.04 & 64.4 \\
\hline 7.777 & 0.424 & 54.470 & 0.06 & 64.4 \\
\hline 10.290 & 0.415 & 40.330 & 0.08 & 64.4 \\
\hline 13.570 & 0.453 & 33.410 & 0.10 & 64.4 \\
\hline 16.390 & 0.456 & 27.830 & 0.12 & 64.5 \\
\hline 19.840 & 0.442 & 22.280 & 0.14 & 64.5 \\
\hline 22.700 & 0.428 & 18.870 & 0.16 & 64.5 \\
\hline 25.620 & 0.442 & 17.240 & 0.18 & 64.5 \\
\hline 28.480 & 0.466 & 16.370 & 0.20 & 64.5 \\
\hline 31.910 & 0.481 & 15.080 & 0.22 & 64.5 \\
\hline 34.770 & 0.488 & 14.030 & 0.24 & 64.5 \\
\hline 37.950 & 0.471 & 12.410 & 0.26 & 64.5 \\
\hline 40.540 & 0.531 & 13.110 & 0.28 & 64.5 \\
\hline 44.010 & 0.502 & 11.410 & 0.30 & 64.6 \\
\hline 47.430 & 0.525 & 11.060 & 0.32 & 64.6 \\
\hline 50.320 & 0.392 & 7.789 & 0.34 & 64.6 \\
\hline 53.260 & 0.544 & 10.210 & 0.36 & 64.6 \\
\hline 56.100 & 0.568 & 10.120 & 0.38 & 64.6 \\
\hline 59.530 & 0.530 & 8.902 & 0.40 & 64.6 \\
\hline 62.410 & 0.629 & 10.070 & 0.42 & 64.6 \\
\hline 65.570 & 0.536 & 8.175 & 0.44 & 64.6 \\
\hline 68.240 & 0.533 & 7.807 & 0.46 & 64.6 \\
\hline 71.650 & 0.572 & 7.989 & 0.48 & 64.6 \\
\hline 74.530 & 0.547 & 7.332 & 0.50 & 64.6 \\
\hline 78.000 & 0.581 & 7.454 & 0.52 & 64.6 \\
\hline 80.480 & 0.640 & 7.953 & 0.54 & 64.6 \\
\hline 83.720 & 0.603 & 7.199 & 0.56 & 64.6 \\
\hline 86.650 & 0.626 & 7.222 & 0.58 & 64.6 \\
\hline 90.070 & 0.581 & 6.445 & 0.61 & 64.6 \\
\hline 92.960 & 0.656 & 7.057 & 0.62 & 64.6 \\
\hline 95.920 & 0.591 & 6.165 & 0.65 & 64.6 \\
\hline 98.760 & 0.584 & 5.916 & 0.66 & 64.6 \\
\hline 102.200 & 0.478 & 4.673 & 0.69 & 64.6 \\
\hline 105.100 & 0.623 & 5.925 & 0.70 & 64.7 \\
\hline 108.200 & 0.624 & 5.762 & 0.73 & 64.7 \\
\hline 110.900 & 0.664 & 5.984 & 0.74 & 64.7 \\
\hline 113.800 & 0.647 & 5.682 & 0.77 & 64.7 \\
\hline 116.700 & 0.742 & 6.357 & 0.78 & 64.7 \\
\hline 120.100 & 0.701 & 5.841 & 0.81 & 64.7 \\
\hline 122.400 & 0.547 & 4.473 & 0.82 & 64.6 \\
\hline 125.800 & 0.670 & 5.324 & 0.85 & 64.7 \\
\hline 128.700 & 0.677 & 5.264 & 0.87 & 64.7 \\
\hline 132.100 & 0.711 & 5.381 & 0.89 & 64.7 \\
\hline 135.000 & 0.706 & 5.232 & 0.91 & 64.7 \\
\hline 137.800 & 0.731 & 5.302 & 0.93 & 64.7 \\
\hline 141.200 & 0.665 & 4.705 & 0.95 & 64.7 \\
\hline 144.100 & 0.661 & 4.586 & 0.97 & 64.7 \\
\hline 147.500 & 0.688 & 4.665 & 0.99 & 64.7 \\
\hline 149.800 & 0.713 & 4.757 & 1.01 & 64.7 \\
\hline 153.300 & 0.675 & 4.404 & 1.03 & 64.7 \\
\hline 156.100 & 0.749 & 4.796 & 1.05 & 64.7 \\
\hline 159.500 & 0.656 & 4.109 & 1.07 & 64.7 \\
\hline 162.400 & 0.668 & 4.112 & 1.09 & 64.7 \\
\hline 165.300 & 0.737 & 4.462 & 1.11 & 64.6 \\
\hline 168.100 & 0.733 & 4.358 & 1.13 & 64.7 \\
\hline 171.600 & 0.693 & 4.039 & 1.15 & 64.7 \\
\hline 174.400 & 0.741 & 4.246 & 1.17 & 64.7 \\
\hline 177.300 & 0.691 & 3.898 & 1.19 & 64.7 \\
\hline 180.100 & 0.731 & 4.057 & 1.21 & 64.7 \\
\hline 183.600 & 0.753 & 4.099 & 1.23 & 64.7 \\
\hline 186.400 & 0.759 & 4.069 & 1.25 & 64.7 \\
\hline 189.900 & 0.719 & 3.789 & 1.27 & 64.7 \\
\hline 192.100 & 0.745 & 3.879 & 1.29 & 64.7 \\
\hline 195.600 & 0.760 & 3.886 & 1.31 & 64.7 \\
\hline
\end{tabular}


RPP-5798, Rev. 0

\begin{tabular}{|c|c|c|c|c|}
\hline $\begin{array}{l}198.400 \\
201.900\end{array}$ & $\begin{array}{l}0.777 \\
0.874\end{array}$ & $\begin{array}{l}3.913 \\
4.331\end{array}$ & $\begin{array}{l}1.33 \\
1.35\end{array}$ & $\begin{array}{l}64.7 \\
64.7\end{array}$ \\
\hline 204.700 & 0.780 & 3.809 & 1.37 & 64.7 \\
\hline 207.600 & 0.761 & 3.668 & 1.39 & 64.7 \\
\hline 210.400 & 0.820 & 3.894 & 1.41 & 64.7 \\
\hline 213.900 & 0.754 & 3.525 & 1.43 & 64.7 \\
\hline 216.700 & 0.811 & 3.740 & 1.45 & 64.7 \\
\hline 219.600 & 0.808 & 3.681 & 1.47 & 64.7 \\
\hline 223.000 & 0.739 & 3.315 & 1.50 & 64.7 \\
\hline 225.900 & 0.794 & 3.515 & 1.51 & 64.7 \\
\hline 229.300 & 0.800 & 3.489 & 1.54 & 64.7 \\
\hline 232.200 & 0.844 & 3.634 & 1.55 & 64.7 \\
\hline 235.000 & 0.752 & 3.198 & 1.58 & 64.7 \\
\hline 237.900 & 0.823 & 3.461 & 1.59 & 64.7 \\
\hline 241.300 & 0.856 & 3.547 & 1.62 & 64.7 \\
\hline 244.200 & 0.826 & 3.381 & 1.63 & 64.7 \\
\hline 247.000 & 0.808 & 3.270 & 1.66 & 64.7 \\
\hline 249.900 & 0.841 & 3.365 & 1.67 & 64.6 \\
\hline 253.300 & 0.855 & 3.373 & 1.70 & 64.7 \\
\hline 256.200 & 0.881 & 3.439 & 1.72 & 64.7 \\
\hline 259.600 & 0.883 & 3.402 & 1.74 & 64.7 \\
\hline 261.900 & 0.847 & 3.234 & 1.76 & 64.7 \\
\hline 265.300 & 0.848 & 3.197 & 1.78 & 64.7 \\
\hline 268.200 & 0.879 & 3.278 & 1.80 & 64.7 \\
\hline 271.600 & 0.853 & 3.139 & 1.82 & 64.7 \\
\hline 274.500 & 0.886 & 3.228 & 1.84 & 64.7 \\
\hline 277.300 & 0.874 & 3.153 & 1.86 & 64.7 \\
\hline 280.200 & 0.822 & 2.933 & 1.88 & 64.7 \\
\hline 283.600 & 1.010 & 3.562 & 1.90 & 64.7 \\
\hline 286.500 & 0.763 & 2.664 & 1.92 & 64.7 \\
\hline 289.400 & 0.855 & 2.953 & 1.94 & 64.7 \\
\hline 292.200 & 0.828 & 2.832 & 1.96 & 64.7 \\
\hline 295.600 & 0.890 & 3.012 & 1.98 & 64.7 \\
\hline 298.500 & 0.852 & 2.855 & 2.00 & 64.7 \\
\hline 300.200 & 0.869 & 2.895 & 2.00 & 64.7 \\
\hline 298.500 & 0.799 & 2.677 & 2.02 & 64.7 \\
\hline 295.800 & 0.796 & 2.692 & 2.04 & 64.7 \\
\hline 292.600 & 0.776 & 2.652 & 2.06 & 64.7 \\
\hline 289.700 & 0.707 & 2.440 & 2.08 & 64.7 \\
\hline 286.300 & 0.771 & 2.694 & 2.10 & 64.7 \\
\hline 283.600 & 0.796 & 2.808 & 2.12 & 64.7 \\
\hline 280.600 & 0.733 & 2.613 & 2.14 & 64.7 \\
\hline 277.700 & 0.701 & 2.522 & 2.16 & 64.7 \\
\hline 274.300 & 0.817 & 2.979 & 2.18 & 64.7 \\
\hline 271.600 & 0.782 & 2.880 & 2.20 & 64.7 \\
\hline 268.600 & 0.740 & 2.756 & 2.22 & 64.7 \\
\hline 265.500 & 0.716 & 2.695 & 2.24 & 64.7 \\
\hline 262.100 & 0.646 & 2.465 & 2.26 & 64.7 \\
\hline 259.200 & 0.706 & 2.722 & 2.28 & 64.7 \\
\hline 256.200 & 0.691 & 2.696 & 2.30 & 64.7 \\
\hline 253.100 & 0.689 & 2.722 & 2.32 & 64.7 \\
\hline 250.300 & 0.599 & 2.393 & 2.34 & 64.7 \\
\hline 246.700 & 0.691 & 2.800 & 2.36 & 64.7 \\
\hline 244.000 & 0.644 & 2.639 & 2.38 & 64.7 \\
\hline 240.900 & 0.754 & 3.129 & 2.40 & 64.7 \\
\hline 238.100 & 0.732 & 3.073 & 2.42 & 64.7 \\
\hline 234.600 & 0.696 & 2.967 & 2.44 & 64.7 \\
\hline 231.800 & 0.648 & 2.796 & 2.46 & 64.7 \\
\hline 228.700 & 0.748 & 3.271 & 2.48 & 64.7 \\
\hline 226.100 & 0.668 & 2.956 & 2.50 & 64.7 \\
\hline 222.800 & 0.675 & 3.027 & 2.53 & 64.7 \\
\hline 220.000 & 0.619 & 2.813 & 2.54 & 64.7 \\
\hline 216.300 & 0.666 & 3.078 & 2.57 & 64.7 \\
\hline 214.100 & 0.640 & 2.988 & 2.58 & 64.7 \\
\hline 210.600 & 0.684 & 3.245 & 2.61 & 64.7 \\
\hline 207.800 & 0.637 & 3.067 & 2.62 & 64.7 \\
\hline 204.300 & 0.559 & 2.737 & 2.65 & 64.7 \\
\hline 201.900 & 0.630 & 3.119 & 2.66 & 64.7 \\
\hline 198.600 & 0.632 & 3.184 & 2.69 & 64.7 \\
\hline 195.800 & 0.614 & 3.134 & 2.70 & 64.7 \\
\hline
\end{tabular}


RPP-5798, Rev. 0

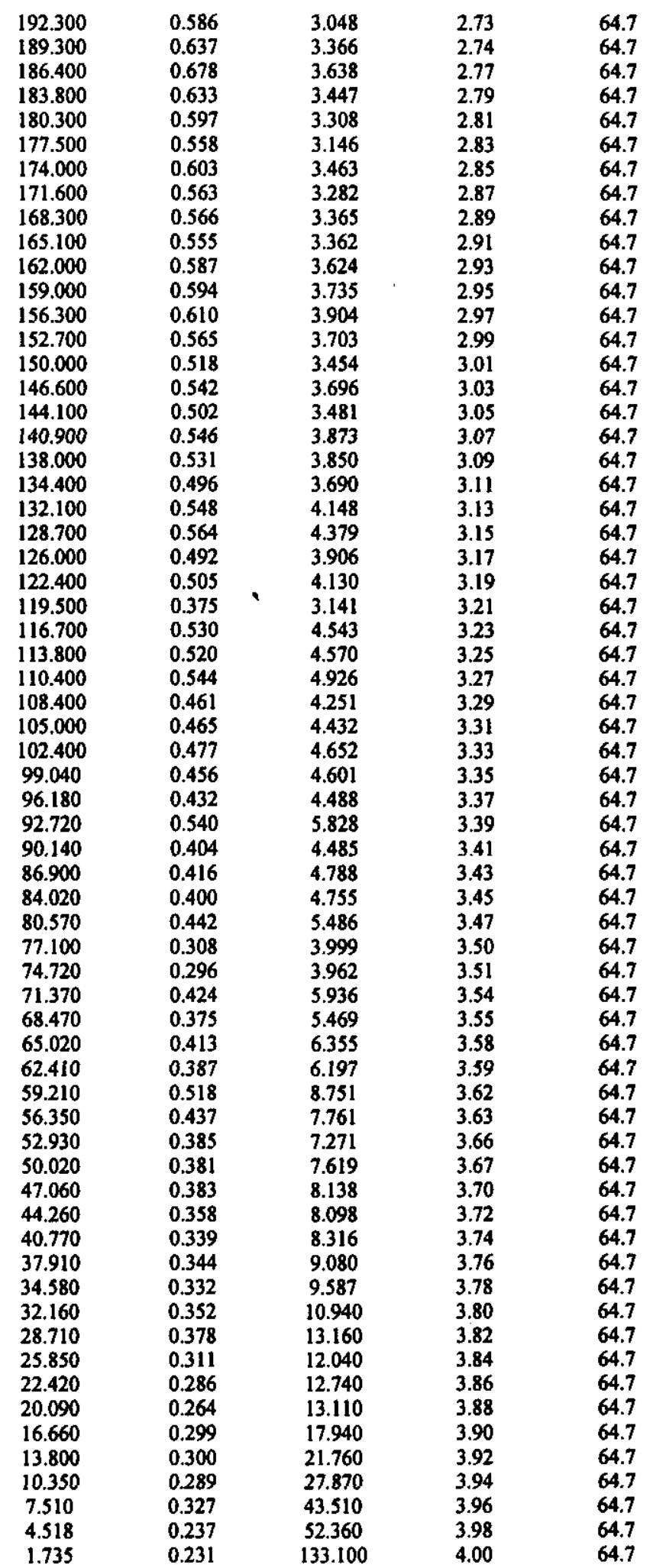


RPP-5798, Rev. 0

\section{VISCOSITY ANALYSIS OF}

\section{HIGH SOLIDS DILUTION (200 g solids/L)}

\section{AT AMBIENT TEMPERATURE}


RPP-5798, Rev. 0

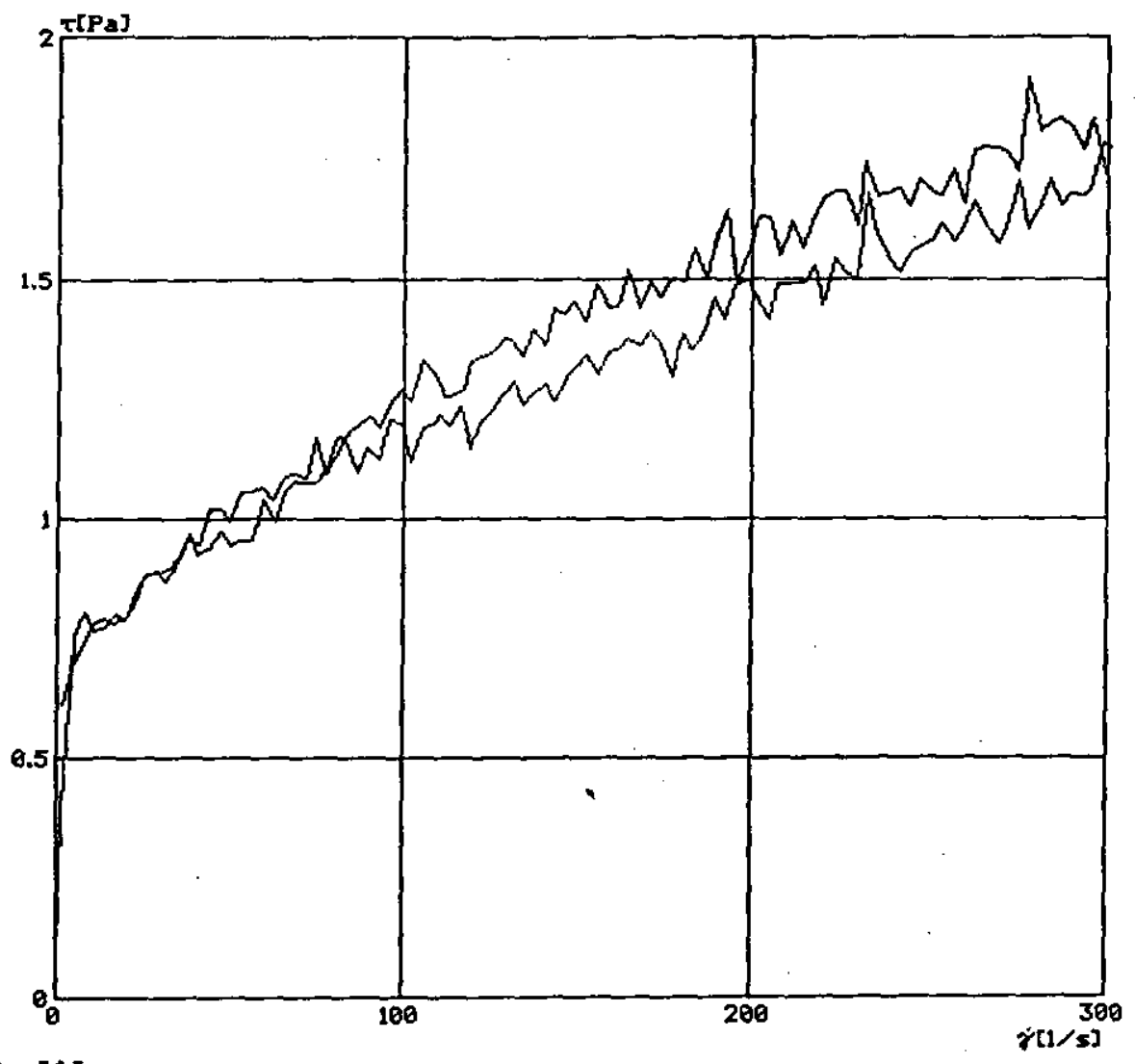

Hคค $ค$ ค $\mathbf{2}$

Operator:

jwc/sy

Substance: c184 200g/1 room tempo

Test No.:

a1

Test of: 68-29-1999

System: M5/MV1

Temper ture: $23.0^{\circ} \mathrm{C}$

C426e1.ROT

WAAKF RAT 3 A 3

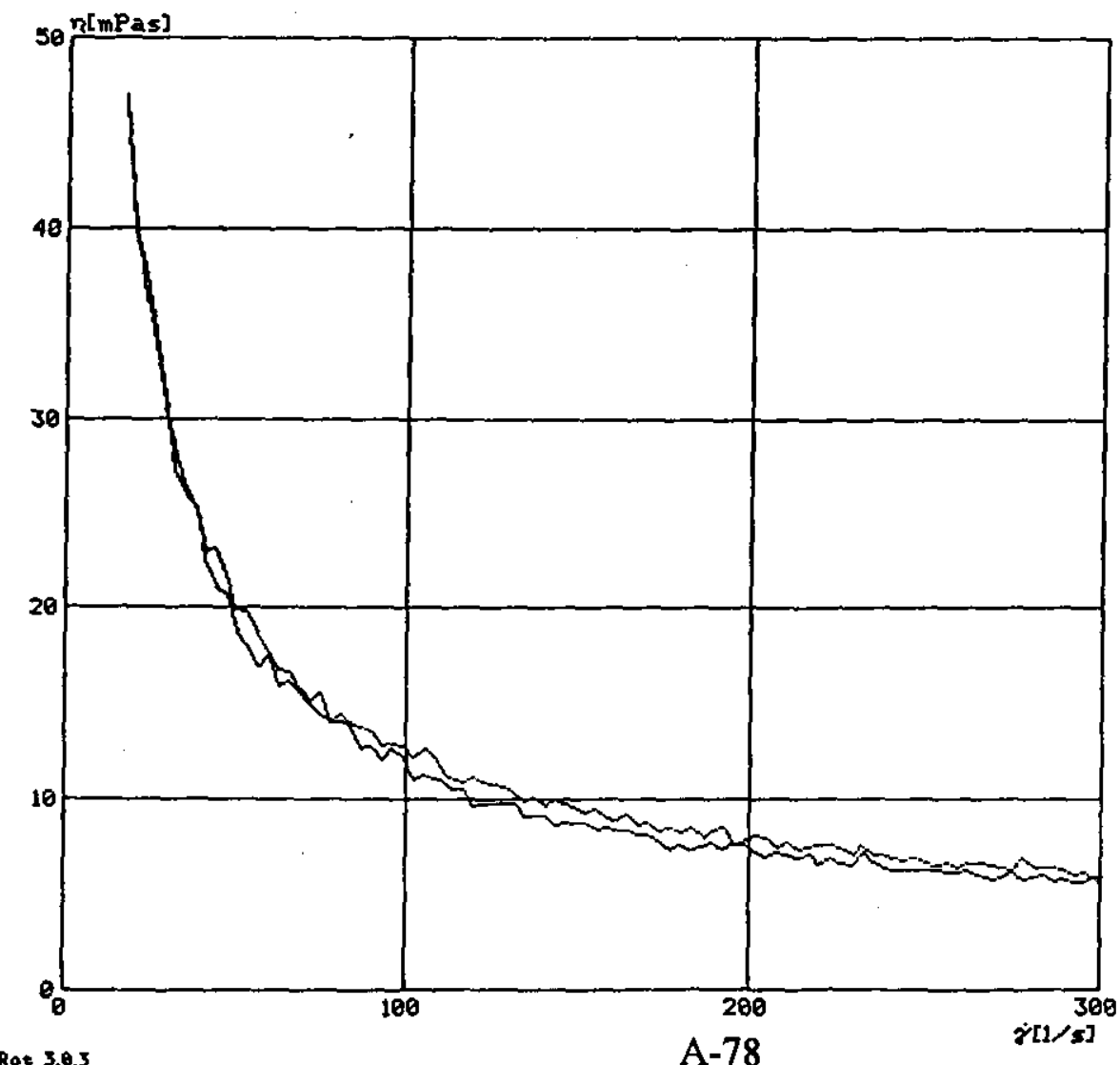

H A A $\mathrm{X}$

Operator: Jwe/gy

Substance: 0104 200g/ room temp Test No.t e1

Text of 08-29-1999

System: M5/MVI

Temperature: $23.8^{\circ} \mathrm{C}$ 
RPP-5798, Rev. 0

\begin{tabular}{|c|c|c|c|c|}
\hline$\tau[\mathbf{P a}]$ & $\gamma\left[s^{-1}\right]$ & $\mathfrak{\eta}[\mathrm{mPas}]$ & $t \mid m i n]$ & $\left.\operatorname{Temp} \mid{ }^{\circ} \mathrm{C}\right]$ \\
\hline 0.000 & 0.077 & 0.000 & 0.00 & 27.9 \\
\hline 1.411 & 0.397 & 281.400 & 0.02 & 27.9 \\
\hline 4.880 & 0.751 & 154.000 & 0.04 & 27.9 \\
\hline 7.339 & 0.800 & 109.100 & 0.06 & 27.9 \\
\hline 10.670 & 0.764 & 71.530 & 0.08 & 27.9 \\
\hline 13.510 & 0.769 & 56.920 & 0.10 & 27.9 \\
\hline 16.980 & 0.800 & 47.070 & 0.12 & 27.9 \\
\hline 19.750 & 0.786 & 39.820 & 0.14 & 27.9 \\
\hline 22.720 & 0.854 & 37.580 & 0.16 & 27.9 \\
\hline 25.600 & 0.886 & 34.590 & 0.18 & 27.9 \\
\hline 29.050 & 0.885 & 30.450 & 0.20 & 27.9 \\
\hline 31.890 & 0.894 & 28.030 & 0.22 & 27.9 \\
\hline 34.960 & 0.920 & 26.300 & 0.24 & 27.9 \\
\hline 37.650 & 0.959 & 25.470 & 0.26 & 27.9 \\
\hline 41.080 & 0.945 & 23.010 & 0.28 & 27.9 \\
\hline 43.960 & 1.020 & 23.200 & 0.30 & 27.9 \\
\hline 47.460 & 1.017 & 21.430 & 0.32 & 27.9 \\
\hline 49.830 & 0.996 & 19.990 & 0.34 & 28.0 \\
\hline 53.220 & 1.055 & 19.820 & 0.36 & 27.9 \\
\hline 56.100 & 1.056 & 18.820 & 0.38 & 27.9 \\
\hline 59.530 & 1.063 & 17.850 & 0.40 & 27.9 \\
\hline 62.140 & 1.041 & 16.750 & 0.42 & 28.0 \\
\hline 65.340 & 1.087 & 16.630 & 0.44 & 27.9 \\
\hline 68.160 & 1.087 & 15.950 & 0.46 & 27.9 \\
\hline 71.630 & 1.086 & 15.160 & 0.48 & 28.0 \\
\hline 74.510 & 1.163 & 15.610 & 0.50 & 28.0 \\
\hline 77.540 & 1.091 & 14.080 & 0.52 & 28.0 \\
\hline 80.340 & 1.127 & 14.030 & 0.54 & 28.0 \\
\hline 83.760 & 1.177 & 14.060 & 0.56 & 28.0 \\
\hline 86.620 & 1.190 & 13.740 & 0.58 & 27.9 \\
\hline 89.990 & 1.212 & 13.470 & 0.60 & 28.0 \\
\hline 93.000 & 1.188 & 12.770 & 0.63 & 28.0 \\
\hline 95.860 & 1.229 & 12.830 & 0.64 & 27.9 \\
\hline 99.330 & 1.267 & 12.760 & 0.67 & 28.0 \\
\hline 102.200 & 1.242 & 12.140 & 0.68 & 28.0 \\
\hline 105.300 & 1.327 & 12.600 & 0.71 & 28.0 \\
\hline 108.000 & 1.304 & 12.070 & 0.73 & 28.0 \\
\hline 111.500 & 1.251 & 11.220 & 0.75 & 27.9 \\
\hline 113.800 & 1.251 & 10.990 & 0.77 & 28.0 \\
\hline 117.200 & 1.268 & 10.820 & 0.79 & 28.0 \\
\hline 119.500 & 1.322 & 11.060 & 0.81 & 27.9 \\
\hline 122.900 & 1.332 & 10.840 & 0.83 & 28.0 \\
\hline 125.800 & 1.344 & 10.690 & 0.85 & 28.0 \\
\hline 129.200 & 1.374 & 10.630 & 0.87 & 28.0 \\
\hline 131.500 & 1.367 & 10.400 & 0.89 & 28.0 \\
\hline 135.000 & 1.330 & 9.852 & 0.91 & 28.0 \\
\hline 137.800 & 1.394 & 10.120 & 0.93 & 28.0 \\
\hline 141.200 & 1.355 & 9.591 & 0.95 & 28.0 \\
\hline 144.100 & 1.435 & 9.961 & 0.97 & 28.0 \\
\hline 147.000 & 1.424 & 9.687 & 0.99 & 28.0 \\
\hline 149.800 & 1.446 & 9.653 & 1.01 & 28.0 \\
\hline 153.300 & 1.409 & 9.191 & 1.03 & 28.0 \\
\hline 156.100 & 1.487 & 9.525 & 1.05 & 28.0 \\
\hline 159.500 & 1.437 & 9.009 & 1.07 & 28.0 \\
\hline 161.800 & 1.435 & 8.870 & 1.09 & 28.0 \\
\hline 165.300 & 1.520 & 9.195 & 1.11 & 28.0 \\
\hline 168.100 & 1.436 & 8.541 & 1.13 & 28.0 \\
\hline 171.600 & 1.493 & 8.703 & 1.15 & 28.0 \\
\hline 173.800 & 1.455 & 8.371 & 1.17 & 28.0 \\
\hline 177.300 & 1.502 & 8.473 & 1.19 & 28.0 \\
\hline 180.700 & 1.494 & 8.270 & 1.21 & 28.0 \\
\hline 183.600 & 1.563 & 8.512 & 1.23 & 28.0 \\
\hline 187.000 & 1.500 & 8.020 & 1.25 & 28.0 \\
\hline 189.300 & 1.575 & 8.322 & 1.27 & 28.0 \\
\hline 192.700 & 1.644 & 8.532 & 1.29 & 28.0 \\
\hline 195.600 & 1.489 & 7.615 & 1.31 & 28.0 \\
\hline
\end{tabular}


RPP-5798, Rev. 0

\begin{tabular}{|c|c|c|c|c|}
\hline 199.000 & 1.561 & 7.845 & 1.33 & 28.0 \\
\hline 201.700 & 1.629 & 8.076 & 1.35 & 28.0 \\
\hline 204.700 & 1.627 & 7.949 & 1.37 & 28.0 \\
\hline 207.600 & 1.549 & 7.464 & 1.39 & 28.0 \\
\hline 211.000 & 1.620 & 7.676 & 1.41 & 28.0 \\
\hline 213.900 & 1.565 & 7.317 & 1.43 & 28.0 \\
\hline 216.700 & 1.619 & 7.469 & 1.45 & 28.0 \\
\hline 219.600 & 1.667 & 7.591 & 1.47 & 28.0 \\
\hline 223.000 & 1.684 & 7.553 & 1.49 & 28.1 \\
\hline 225.900 & 1.686 & 7.463 & 1.51 & 28.1 \\
\hline 228.900 & 1.613 & 7.044 & 1.53 & 28.0 \\
\hline 231.600 & 1.744 & 7.532 & 1.55 & 28.0 \\
\hline 235.000 & 1.676 & 7.131 & 1.58 & 28.0 \\
\hline 237.900 & 1.679 & 7.057 & 1.59 & 28.0 \\
\hline 241.300 & 1.692 & 7.009 & 1.62 & 28.0 \\
\hline 243.600 & 1.657 & 6.800 & 1.63 & 28.1 \\
\hline 247.000 & 1.708 & 6.912 & 1.66 & 28.0 \\
\hline 249.900 & 1.682 & 6.731 & 1.67 & 28.0 \\
\hline 253.300 & 1.675 & 6.614 & 1.70 & 28.0 \\
\hline 256.200 & 1.728 & 6.746 & 1.71 & 28.1 \\
\hline 259.000 & 1.660 & 6.408 & 1.74 & 28.0 \\
\hline 261.900 & 1.769 & 6.753 & 1.75 & 28.0 \\
\hline 265.300 & 1.773 & 6.683 & 1.78 & 28.1 \\
\hline 268.800 & 1.776 & 6.608 & 1.80 & 28.0 \\
\hline 271.200 & 1.764 & 6.504 & 1.82 & 28.0 \\
\hline 274.500 & 1.726 & 6.290 & 1.84 & 28.1 \\
\hline 277.300 & 1.916 & 6.908 & 1.86 & 28.0 \\
\hline 280.800 & 1.811 & 6.450 & 1.88 & 28.0 \\
\hline 283.600 & 1.826 & 6.438 & 1.90 & 28.1 \\
\hline 286.500 & 1.838 & 6.415 & 1.92 & 28.0 \\
\hline 289.400 & 1.820 & 6.292 & 1.94 & 28.1 \\
\hline 292.800 & 1.770 & 6.047 & 1.96 & 28.1 \\
\hline 295.600 & 1.838 & 6.217 & 1.98 & 28.0 \\
\hline 298.900 & 1.721 & 5.759 & 2.00 & 28.0 \\
\hline 300.200 & 1.777 & 5.918 & 2.00 & 28.0 \\
\hline 298.500 & 1.787 & 5.988 & 2.02 & 28.1 \\
\hline 295.300 & 1.688 & 5.718 & 2.04 & 28.0 \\
\hline 292.600 & 1.676 & 5.729 & 2.06 & 28.1 \\
\hline 289.400 & 1.681 & 5.808 & 2.08 & 28.1 \\
\hline 286.900 & 1.652 & 5.760 & 2.10 & 28.0 \\
\hline 283.400 & 1.711 & 6.038 & 2.12 & 28.1 \\
\hline 280.800 & 1.658 & 5.907 & 2.14 & 28.1 \\
\hline 277.200 & 1.605 & 5.791 & 2.16 & 28.1 \\
\hline 274.500 & 1.706 & 6.216 & 2.18 & 28.1 \\
\hline 271.400 & 1.609 & 5.929 & 2.20 & 28.1 \\
\hline 268.800 & 1.575 & 5.859 & 2.22 & 28.1 \\
\hline 265.100 & 1.619 & 6.107 & 2.24 & 28.1 \\
\hline 262.300 & 1.666 & 6.350 & 2.26 & 28.1 \\
\hline 259.200 & 1.607 & 6.201 & 2.28 & 28.1 \\
\hline 256.600 & 1.577 & 6.145 & 2.30 & 28.1 \\
\hline 253.100 & 1.616 & 6.385 & 2.32 & 28.1 \\
\hline 250.300 & 1.581 & 6.317 & 2.34 & 28.1 \\
\hline 247.000 & 1.567 & 6.344 & 2.36 & 28.1 \\
\hline 244.400 & 1.561 & 6.386 & 2.38 & 28.1 \\
\hline 241.100 & 1.515 & 6.284 & 2.40 & 28.1 \\
\hline 238.100 & 1.538 & 6.462 & 2.42 & 28.1 \\
\hline 234.800 & I.587 & 6.756 & 2.44 & 28.1 \\
\hline 232.200 & 1.681 & 7.239 & 2.46 & 28.1 \\
\hline 228.900 & 1.498 & 6.545 & 2.48 & 28.1 \\
\hline 226.100 & 1.511 & 6.684 & 2.50 & 28.1 \\
\hline 222.600 & 1.546 & 6.942 & 2.52 & 28.1 \\
\hline 219.800 & 1.443 & 6.568 & 2.54 & 28.1 \\
\hline 217.100 & 1.528 & 7.036 & 2.56 & 28.1 \\
\hline 213.700 & 1.489 & 6.970 & 2.59 & 28.1 \\
\hline 210.600 & 1.489 & 7.071 & 2.60 & 28.1 \\
\hline 207.200 & 1.486 & 7.174 & 2.63 & 28.1 \\
\hline 204.700 & 1.413 & 6.901 & 2.64 & 28.1 \\
\hline 201.500 & 1.455 & 7.221 & 2.67 & 28.1 \\
\hline 198.600 & 1.499 & 7.548 & 2.69 & 28.1 \\
\hline 195.200 & 1.484 & 7.603 & 2.71 & 28.1 \\
\hline
\end{tabular}


RPP-5798, Rev. 0

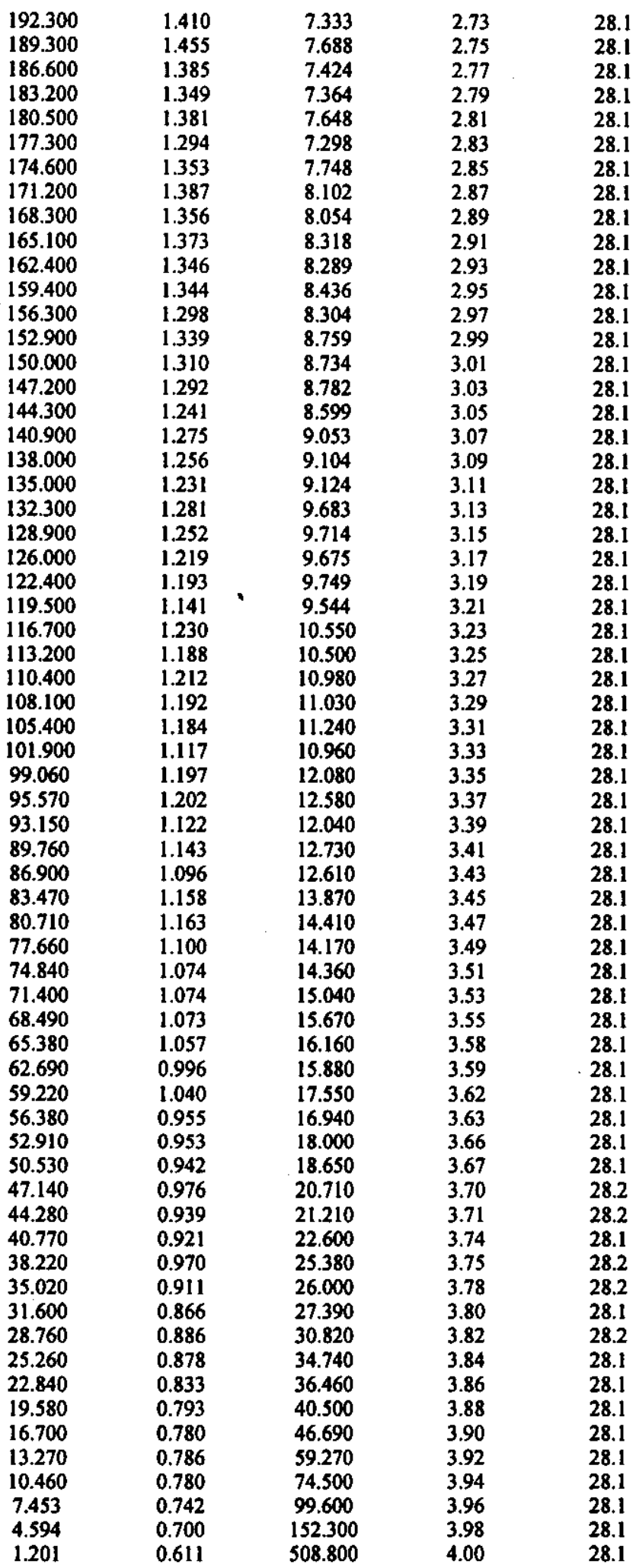


RPP-5798, Rev. 0

\section{VISCOSITY ANALYSIS OF}

HIGH SOLIDS DILUTION (200 g solids/L) AT $45^{\circ} \mathrm{C}$ 
RPP-5798, Rev. 0

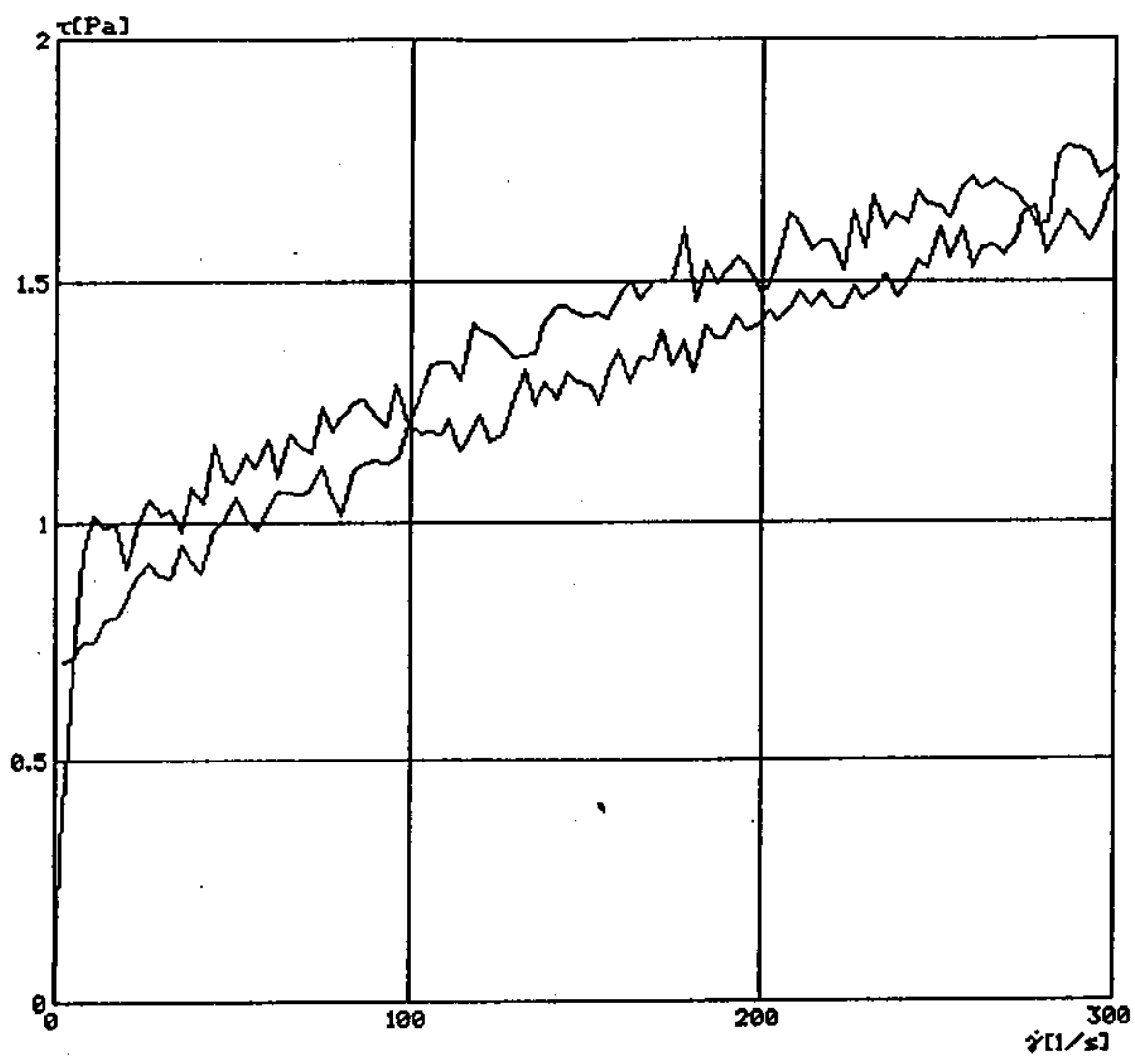

Hค $ค$ ×

Operator:

jwe/gy

Substance:

0104 200g/1

$45 c$

Test No:

o1

Test of:

08-29-1999

System:

MS/MVI

Temperature:

$23.0^{\circ} \mathrm{C}$

HAAKE Rot 3.0.3

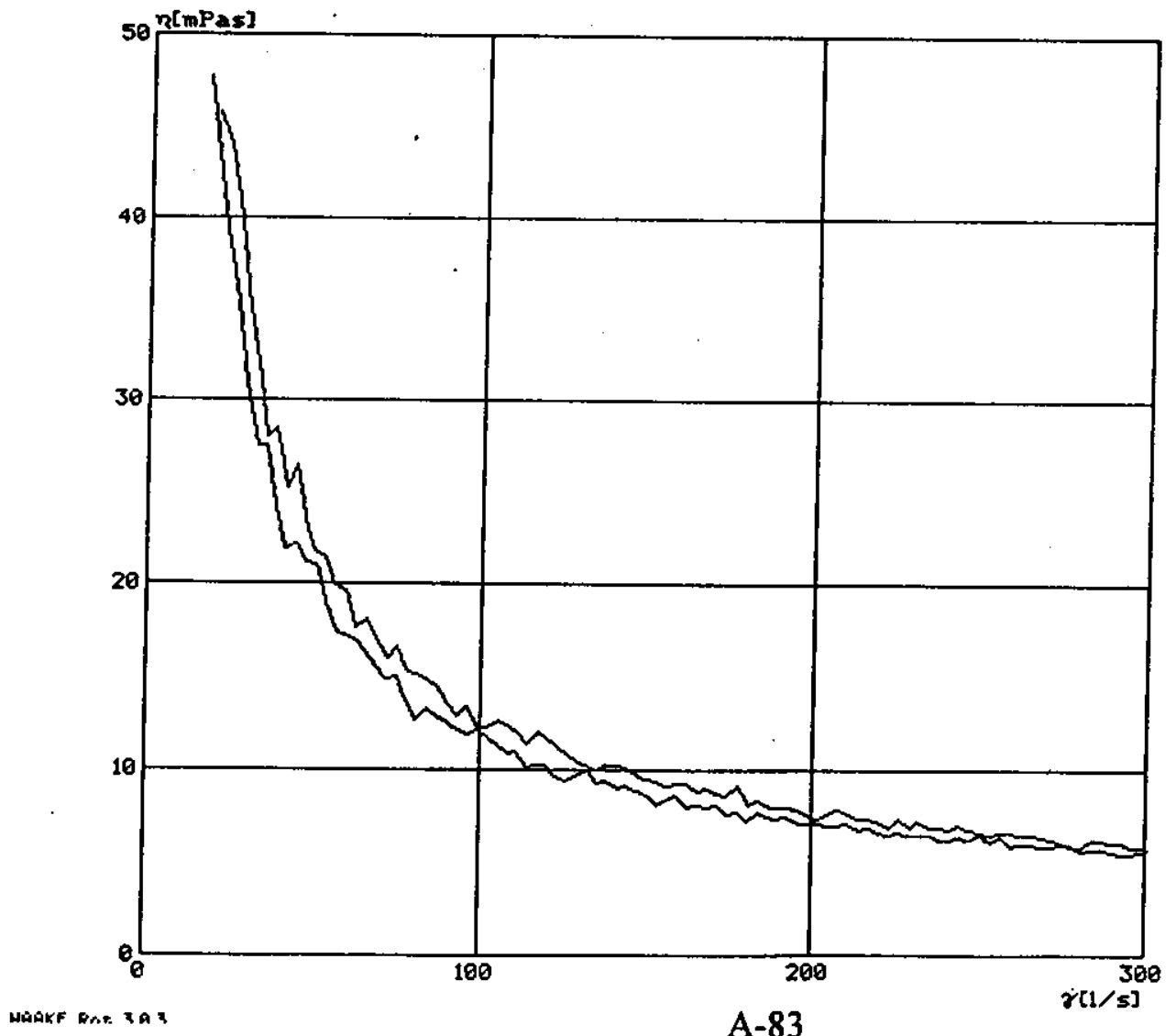

H $\boldsymbol{A} \boldsymbol{A} \boldsymbol{I}$

Operator:

jwe/gy

Substance:

c104 2009/1

450

Test No.:

e1

Test of:

08-29-1999

System:

MS/MV1

Temperature:

$23.0^{\circ} \mathrm{C}$

C42002.ROT 
RPP-5798, Rev. 0

\begin{tabular}{|c|c|c|c|c|}
\hline T [Pa] & $\left.\gamma \mid s^{-1}\right]$ & $\eta[m$ Pas] & $t[\mathrm{~min}]$ & $\operatorname{Temp}\left[{ }^{\circ} \mathrm{C}\right]$ \\
\hline 0.000 & 0.000 & 0.000 & 0.00 & 45.0 \\
\hline 1.449 & 0.317 & 218.800 & 0.02 & 45.0 \\
\hline 4.842 & 0.691 & 142.800 & 0.04 & 45.0 \\
\hline 7.301 & 0.941 & 128.800 & 0.06 & 44.9 \\
\hline 10.670 & 1.012 & 94.810 & 0.08 & 44.9 \\
\hline 13.500 & 0.987 & 73.100 & 0.10 & 44.9 \\
\hline 16.980 & 0.996 & 58.670 & 0.12 & 44.9 \\
\hline 19.710 & 0.903 & 45.810 & 0.14 & 44.9 \\
\hline 22.760 & 1.000 & 43.950 & 0.16 & 45.0 \\
\hline 25.600 & 1.050 & 41.030 & 0.18 & 44.9 \\
\hline 29.030 & 1.013 & 34.910 & 0.20 & 44.9 \\
\hline 31.890 & 1.022 & 32.050 & 0.22 & 45.0 \\
\hline 34.960 & 0.979 & 28.000 & 0.24 & 45.0 \\
\hline 37.670 & 1.076 & 28.560 & 0.26 & 45.0 \\
\hline 41.120 & 1.038 & 25,260 & 0.28 & 45.0 \\
\hline 43.980 & 1.163 & 26.450 & 0.30 & 44.9 \\
\hline 47.410 & 1.089 & 22.960 & 0.32 & 44.9 \\
\hline 49.830 & 1.087 & 21.810 & 0.34 & 45.0 \\
\hline 53.220 & 1.144 & 21.490 & 0.36 & 44.9 \\
\hline 56.060 & 1.115 & 19.900 & 0.38 & 45.0 \\
\hline 59.570 & 1.175 & 19.730 & 0.40 & 45.0 \\
\hline 62.140 & 1.094 & 17.610 & 0.42 & 45.0 \\
\hline 65.360 & 1.184 & 18.110 & 0.44 & 45.0 \\
\hline 68.200 & 1.160 . & 17.010 & 0.46 & 45.0 \\
\hline 71.670 & 1.147 & 16.010 & 0.48 & 45.0 \\
\hline 74.490 & 1.242 & 16.670 & 0.50 & 45.0 \\
\hline 77.500 & 1.189 & 15.340 & 0.52 & 45.0 \\
\hline 80.310 & 1.221 & 15.200 & 0.54 & 45.0 \\
\hline 83.780 & 1.252 & 14.950 & 0.56 & 45.0 \\
\hline 86.630 & 1.257 & 14.510 & 0.58 & 45.0 \\
\hline 89.990 & 1.219 & 13.550 & 0.60 & 45.0 \\
\hline 93.000 & 1.201 & 12.920 & 0.63 & 45.0 \\
\hline 95.880 & 1.288 & 13.430 & 0.64 & 45.0 \\
\hline 99.310 & 1.204 & 12.130 & 0.67 & 45.0 \\
\hline 102.200 & 1.255 & 12.280 & 0.68 & 45.0 \\
\hline 105.200 & 1.328 & 12.620 & 0.71 & 45.0 \\
\hline 108.000 & 1.333 & 12.340 & 0.73 & 45.0 \\
\hline 111.300 & 1.333 & 11.980 & 0.75 & 45.0 \\
\hline 113.800 & 1.296 & 11.390 & 0.77 & 45,0 \\
\hline 117.200 & 1.413 & 12.050 & 0.79 & 45,0 \\
\hline 119.500 & 1.398 & 11.700 & 0.81 & 45.0 \\
\hline 122.900 & 1.390 & 11.300 & 0.83 & 45.0 \\
\hline 125.800 & 1.365 & 10.850 & 0.85 & 45.0 \\
\hline 129.200 & 1.343 & 10.390 & 0.87 & 45.0 \\
\hline 131.700 & 1.346 & 10.220 & 0.89 & 45.0 \\
\hline 135.000 & 1.352 & 10.020 & 0.91 & 45.0 \\
\hline 137.800 & 1.418 & 10.290 & 0.93 & 45.0 \\
\hline 141.200 & 1.447 & 10.240 & 0.95 & 45.0 \\
\hline 144.100 & 1.449 & 10.060 & 0.97 & 45.0 \\
\hline 147.000 & 1.432 & 9.744 & 0.99 & 45.0 \\
\hline 149.800 & 1.427 & 9.523 & 1.01 & 45.0 \\
\hline 153.300 & 1.433 & 9.353 & 1.03 & 45.0 \\
\hline 156.100 & 1.423 & 9.113 & 1.05 & 45.0 \\
\hline 159.500 & 1.478 & 9.266 & 1.07 & 45.0 \\
\hline 161.800 & 1.498 & 9.255 & 1.09 & 45.0 \\
\hline 165.300 & 1.462 & 8.845 & 1.11 & 45.0 \\
\hline 168.100 & 1.500 & 8.920 & 1.13 & 45.0 \\
\hline 171.600 & 1.498 & 8.730 & 1.15 & 45.0 \\
\hline 173.800 & 1.500 & 8.629 & 1.17 & 45.0 \\
\hline 177.300 & 1.608 & 9.070 & 1.19 & 45.0 \\
\hline 180.700 & 1.460 & 8.079 & 1.21 & 45.0 \\
\hline 183.600 & 1.539 & 8.386 & 1.23 & 45.0 \\
\hline 187.000 & 1.493 & 7.987 & 1.25 & 45.0 \\
\hline 189.300 & 1.516 & 8.010 & 1.27 & 45.0 \\
\hline 192.700 & 1.547 & 8.027 & 1.29 & 45.0 \\
\hline 195.600 & 1.535 & 7.847 & 1.31 & 45.0 \\
\hline
\end{tabular}


RPP-5798, Rev. 0

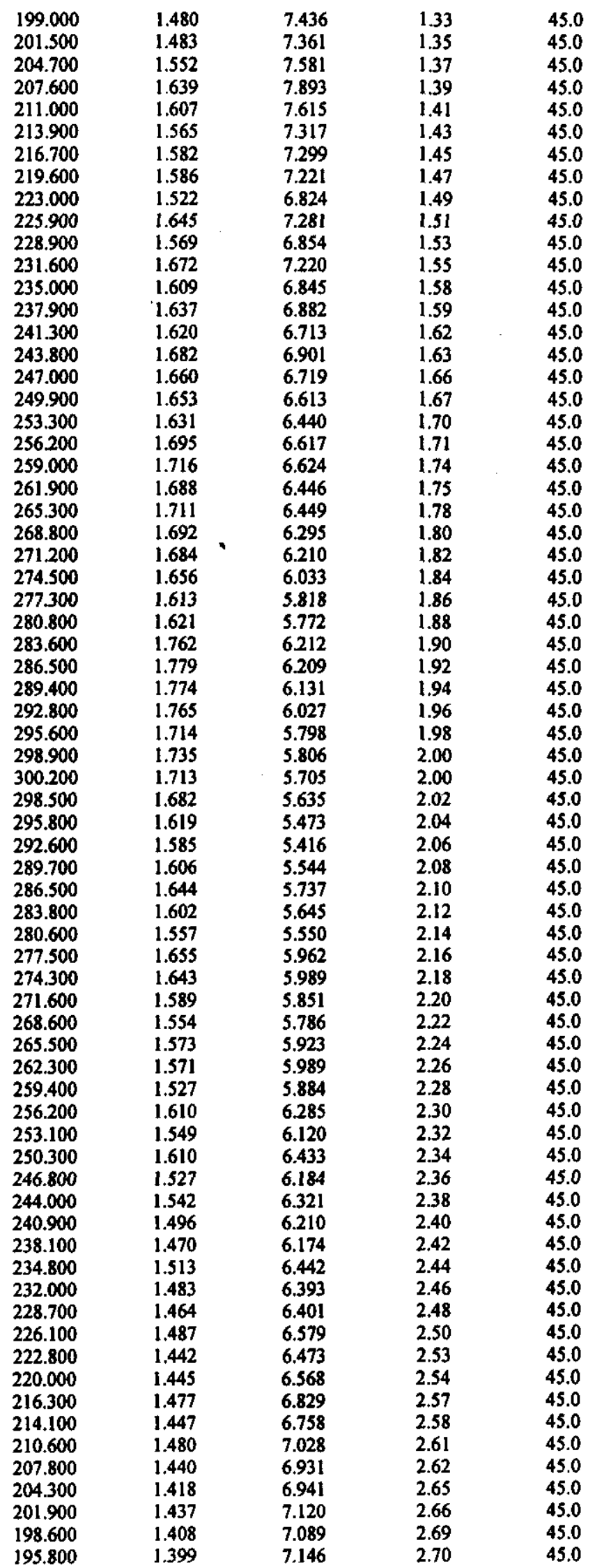


RPP-5798, Rev. 0

\begin{tabular}{|c|c|c|c|c|}
\hline 192.300 & 1.426 & 7.416 & 2.73 & 45.0 \\
\hline 189.500 & 1.381 & 7.291 & 2.74 & 45.0 \\
\hline 186.400 & 1.383 & 7.421 & 2.77 & 45.0 \\
\hline 183.800 & 1.409 & 7.667 & 2.79 & 45.0 \\
\hline 180.300 & 1.310 & 7.266 & 2.81 & 45.0 \\
\hline 177.500 & 1.377 & 7.758 & 2.83 & 45.0 \\
\hline 174.200 & 1.326 & 7.609 & 2.85 & 45.0 \\
\hline 171.600 & 1.397 & 8.141 & 2.87 & 45.0 \\
\hline 168.300 & 1.336 & 7.936 & 2.89 & 45.0 \\
\hline 164.900 & 1.342 & 8.141 & 2.91 & 45.0 \\
\hline 162.200 & 1.291 & 7.957 & 2.93 & 45.0 \\
\hline 159.000 & 1.359 & 8.550 & 2.95 & 45.0 \\
\hline 156.500 & 1.328 & 8.484 & 2.97 & 45.0 \\
\hline 153.100 & 1.246 & 8.140 & 2.99 & 45.0 \\
\hline 150.000 & 1.286 & 8.573 & 3.01 & 45.0 \\
\hline 146.600 & 1.293 & 8.822 & 3.03 & 45.0 \\
\hline 144.100 & 1.311 & 9.095 & 3.05 & 45.0 \\
\hline 140.900 & 1.256 & 8.915 & 3.07 & 45.0 \\
\hline 138.000 & 1.294 & 9.374 & 3.09 & 45.0 \\
\hline 134.600 & 1.246 & 9.262 & 3.11 & 45.0 \\
\hline 132.100 & 1.319 & 9.982 & 3.13 & 45.0 \\
\hline 128.900 & 1.245 & 9.662 & 3.15 & 45.0 \\
\hline 126.000 & 1.184 & 9.401 & 3.17 & 45.0 \\
\hline 122.600 & 1.170 & 9.548 & 3.19 & 45.0 \\
\hline 119.700 & 1.224 & 10.220 & 3.21 & 45.0 \\
\hline 116.700 & 1.193 & 10.220 & 3.23 & 45.0 \\
\hline 113.800 & 1.148 & 10.090 & 3.25 & 45.0 \\
\hline 110.400 & 1.214 & 11.000 & 3.27 & 45.0 \\
\hline 108.400 & 1.184 & 10.910 & 3.29 & 45.0 \\
\hline 104.900 & 1.191 & 11.350 & 3.31 & 45.0 \\
\hline 102.500 & 1.188 & 11.590 & 3.33 & 45.0 \\
\hline 99.020 & 1.204 & 12.160 & 3.35 & 45.0 \\
\hline 96.130 & 1.137 & 11.830 & 3.37 & 45.0 \\
\hline 92.700 & 1.126 & 12.150 & 3.39 & 45.0 \\
\hline 90.200 & 1.129 & 12.520 & 3.41 & 45.0 \\
\hline 86.920 & 1.124 & 12.930 & 3.43 & 45.0 \\
\hline 84.060 & 1.111 & 13.210 & 3.45 & 45.0 \\
\hline 80.540 & 1.014 & 12.590 & 3.47 & 45.0 \\
\hline 77.090 & 1.070 & 13.880 & 3.50 & 45.0 \\
\hline 74.720 & 1.119 & 14.980 & 3.51 & 45.0 \\
\hline 71.330 & 1.064 & 14.920 & 3.54 & 45.0 \\
\hline 68.470 & 1.060 & 15.480 & 3.55 & 45.0 \\
\hline 65.000 & 1.064 & 16.380 & 3.58 & 45.0 \\
\hline 62.430 & 1.062 & 17.010 & 3.59 & 45.0 \\
\hline 59.220 & 1.027 & 17.340 & 3.62 & 45.0 \\
\hline 56.370 & 0.983 & 17.440 & 3.63 & 45.0 \\
\hline 52.910 & 1.011 & 19.110 & 3.66 & 45.0 \\
\hline 50.040 & 1.056 & 21.110 & 3.67 & 45.0 \\
\hline 47.100 & 0.998 & 21.190 & 3.70 & 45.0 \\
\hline 44.260 & 0.983 & 22.200 & 3.72 & 45.0 \\
\hline 40.790 & 0.894 & 21.910 & 3.74 & 45.0 \\
\hline 37.930 & 0.918 & 24.190 & 3.76 & 45.0 \\
\hline 34.600 & 0.953 & 27.540 & 3.78 & 45.0 \\
\hline 32.140 & 0.885 & 27.540 & 3.80 & 45.0 \\
\hline 28.730 & 0.888 & 30.920 & 3.82 & 45.0 \\
\hline 25.850 & 0.912 & 35.280 & 3.84 & 45.0 \\
\hline 22.420 & 0.882 & 39.330 & 3.86 & 45.0 \\
\hline 19.920 & 0.847 & 42.500 & 3.88 & 45.0 \\
\hline 16.660 & 0.796 & 47.790 & 3.90 & 45.0 \\
\hline 13.800 & 0.794 & 57.550 & 3.92 & 45.0 \\
\hline 10.290 & 0.746 & 72.430 & 3.94 & 45.0 \\
\hline 7.472 & 0.745 & 99.710 & 3.96 & 45.0 \\
\hline 4.575 & 0.713 & 155.800 & 3.98 & 45.0 \\
\hline 1.773 & 0.708 & 399.500 & 4.00 & 45.0 \\
\hline
\end{tabular}


RPP-5798, Rev. 0

\section{VISCOṠITY ANALYSIS OF}

HIGH SOLIDS DILUTION (200 g solids/L) AT $65^{\circ} \mathrm{C}$ 
RPP-5798, Rev. 0

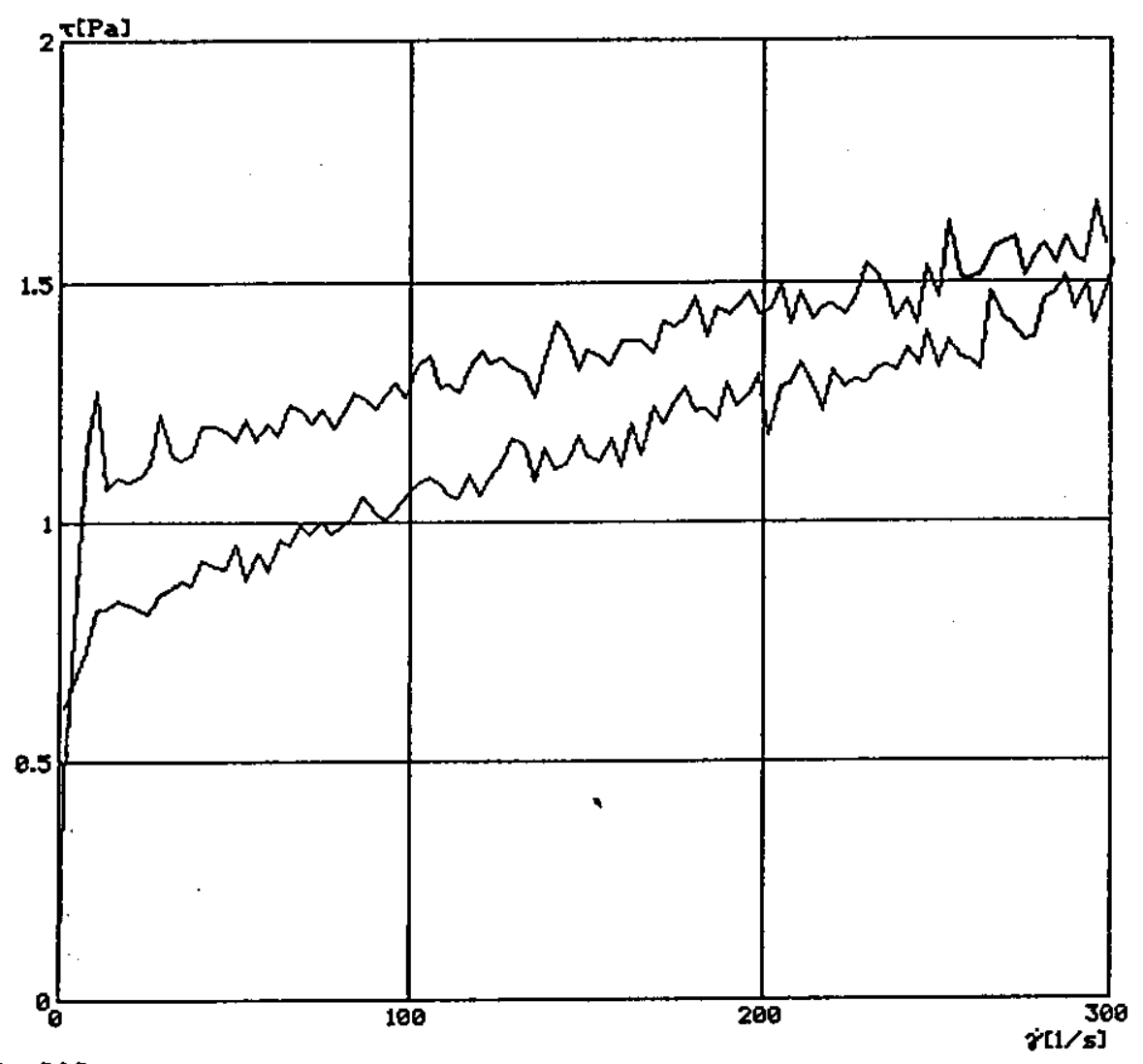

H ค ค $\mathrm{K} \mathrm{E}$

Operator:

jwo/sy

Substance:

c104 200g/1

650

Test No.?

Di

Test of:

08-29-1999

System:

MS/MVI

Temperature:

$23.0^{\circ} \mathrm{C}$

HAAKE Rot 3.0.5

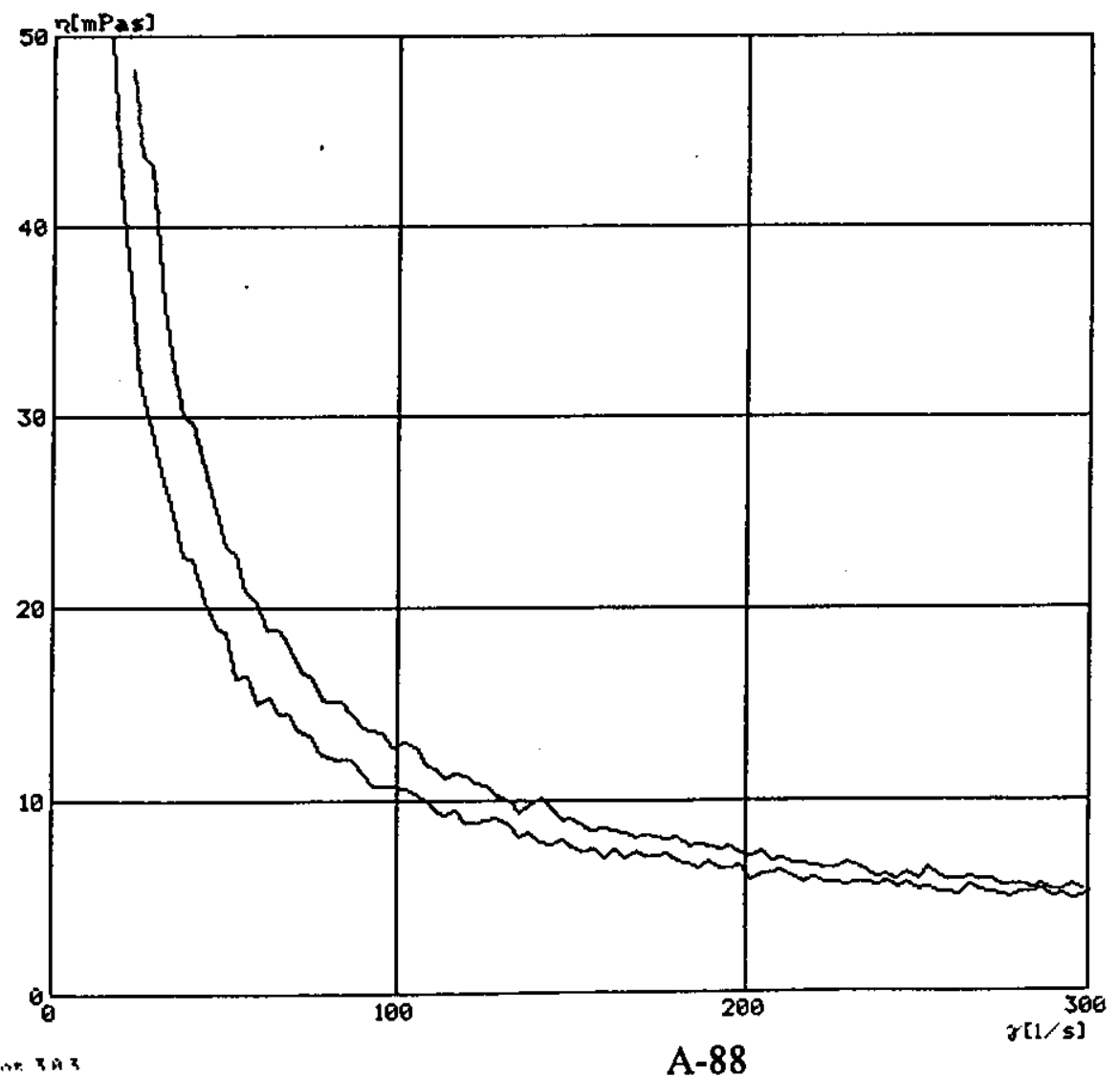

H ค ค $K E$

Oyerator:

jwe/gy

Substance:

clü4 200g/l

650

Test No.:

01

Test of:

08-29-1999

System:

M5/MV1

Temperature:

$23.8^{\circ} \mathrm{C}$ 
RPP-5798, Rev. 0

\begin{tabular}{|c|c|c|c|c|}
\hline$\tau$ [Pa] & $\gamma\left[s^{-1}\right]$ & $\eta[\mathrm{mPas}]$ & t|min] & Temp $\left|{ }^{\circ} \mathrm{C}\right|$ \\
\hline 0.000 & 0.069 & 0.000 & 0.00 & 64.6 \\
\hline 1.391 & 0.457 & 328.600 & 0.02 & 64.6 \\
\hline 4.289 & 0.766 & 178.700 & 0.04 & 64.6 \\
\hline 7.777 & 1.155 & 148.500 & 0.06 & 64.6 \\
\hline 10.260 & 1.275 & 124.300 & 0.08 & 64.6 \\
\hline 13.530 & 1.071 & 79.100 & 0.10 & 64.6 \\
\hline 16.390 & 1.092 & 66.630 & 0.12 & 64.6 \\
\hline 19.800 & 1.085 & 54.800 & 0.14 & 64.6 \\
\hline 22.700 & 1.096 & 48.280 & 0.16 & 64.6 \\
\hline 25.680 & 1.119 & 43.570 & 0.18 & 64.6 \\
\hline 28.460 & 1.227 & 43.130 & 0.20 & 64.6 \\
\hline 31.890 & 1.141 & 35.790 & 0.22 & 64.6 \\
\hline 34.750 & 1.130 & 32.510 & 0.24 & 64.6 \\
\hline 37.950 & 1.139 & 30.010 & 0.26 & 64.6 \\
\hline 40.560 & 1.202 & 29.640 & 0.28 & 64.6 \\
\hline 43.980 & 1.203 & 27.350 & 0.30 & 64.6 \\
\hline 47.430 & 1.190 & 25.080 & 0.32 & 64.6 \\
\hline 50.320 & 1.170 & 23.250 & 0.34 & 64.6 \\
\hline 53.300 & 1.218 & 22.850 & 0.36 & 64.6 \\
\hline 56.060 & 1.171 & 20.890 & 0.38 & 64.6 \\
\hline 59.530 & 1.207 & 20.270 & 0.40 & 64.6 \\
\hline 62.410 & 1.179 & 18.900 & 0.42 & 64.6 \\
\hline 65.590 & 1.243 & 18.960 & 0.44 & 64.7 \\
\hline 68.240 & 1.237 & 18.130 & 0.46 & 64.6 \\
\hline 71.690 & 1.207 & 16.830 & 0.48 & 64.6 \\
\hline 74.530 & 1.234 & 16.560 & 0.50 & 64.7 \\
\hline 78.000 & 1.194 & 15.310 & 0.52 & 64.6 \\
\hline 80.460 & 1.218 & 15.140 & 0.54 & 64.6 \\
\hline 83.780 & 1.272 & 15.180 & 0.56 & 64.7 \\
\hline 86.620 & 1.261 & 14.560 & 0.58 & 64.7 \\
\hline 90.120 & 1.237 & 13.730 & 0.61 & 64.6 \\
\hline 93.000 & 1.264 & 13.590 & 0.62 & 64.6 \\
\hline 95.900 & 1.291 & 13.460 & 0.65 & 64.6 \\
\hline 98.780 & 1.260 & 12.750 & 0.66 & 64.6 \\
\hline 102.200 & 1.324 & 12.960 & 0.69 & 64.6 \\
\hline 105.200 & 1.348 & 12.820 & 0.70 & 64.6 \\
\hline 108.200 & 1.279 & 11.820 & 0.73 & 64.6 \\
\hline 110.900 & 1.287 & 11.610 & 0.74 & 64.6 \\
\hline 113.800 & 1.270 & 11.160 & 0.77 & 64.7 \\
\hline 116.700 & 1.322 & 11.330 & 0.78 & 64.7 \\
\hline 120.100 & 1.356 & 11.290 & 0.81 & 64.6 \\
\hline 122.400 & 1.330 & 10.870 & 0.82 & 64.7 \\
\hline 125.800 & 1.344 & 10.680 & 0.85 & 64.7 \\
\hline 128.700 & 1.322 & 10.280 & 0.87 & 64.6 \\
\hline 132.100 & 1.313 & 9.939 & 0.89 & 64.6 \\
\hline 135.100 & 1.259 & 9.316 & 0.91 & 64.7 \\
\hline 137.800 & 1.338 & 9.712 & 0.93 & 64.7 \\
\hline 141.200 & 1.419 & 10.050 & 0.95 & 64.7 \\
\hline 144.100 & 1.388 & 9.629 & 0.97 & 64.7 \\
\hline 147.500 & 1.318 & 8.931 & 0.99 & 64.7 \\
\hline 149.800 & 1.355 & 9.044 & 1.01 & 64.7 \\
\hline 153.300 & 1.346 & 8.780 & 1.03 & 64.7 \\
\hline 156.100 & 1.328 & 8.507 & 1.05 & 64.7 \\
\hline 159.500 & 1.379 & 8.641 & 1.07 & 64.7 \\
\hline 162.400 & 1.379 & 8.492 & 1.09 & 64.7 \\
\hline 165.300 & 1.375 & 8.319 & 1.11 & 64.7 \\
\hline 168.100 & 1.351 & 8.037 & 1.13 & 64.7 \\
\hline 171.600 & 1.415 & 8.248 & 1.15 & 64.7 \\
\hline 174.400 & 1.406 & 8.061 & 1.17 & 64.7 \\
\hline 177.300 & 1.423 & 8.025 & 1.19 & 64.7 \\
\hline 180.100 & 1.466 & 8.136 & 1.21 & 64.7 \\
\hline 183.600 & 1.387 & 7.557 & 1.23 & 64.7 \\
\hline 186.400 & 1.448 & 7.768 & 1.25 & 64.7 \\
\hline 189.900 & 1.431 & 7.538 & 1.27 & 64.7 \\
\hline 192.100 & 1.446 & 7.524 & 1.29 & 64.7 \\
\hline 195.600 & 1.476 & 7.549 & 1.31 & 64.7 \\
\hline
\end{tabular}


RPP-5798, Rev. 0

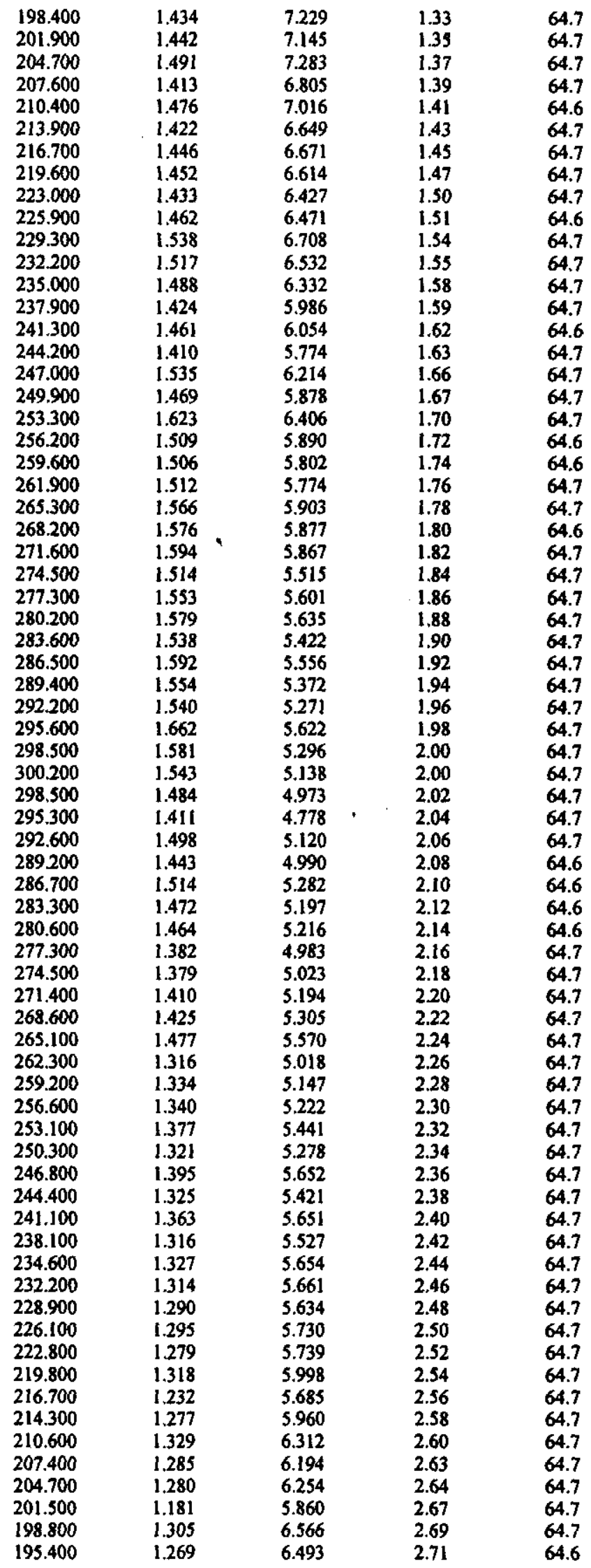


RPP-5798, Rev. 0

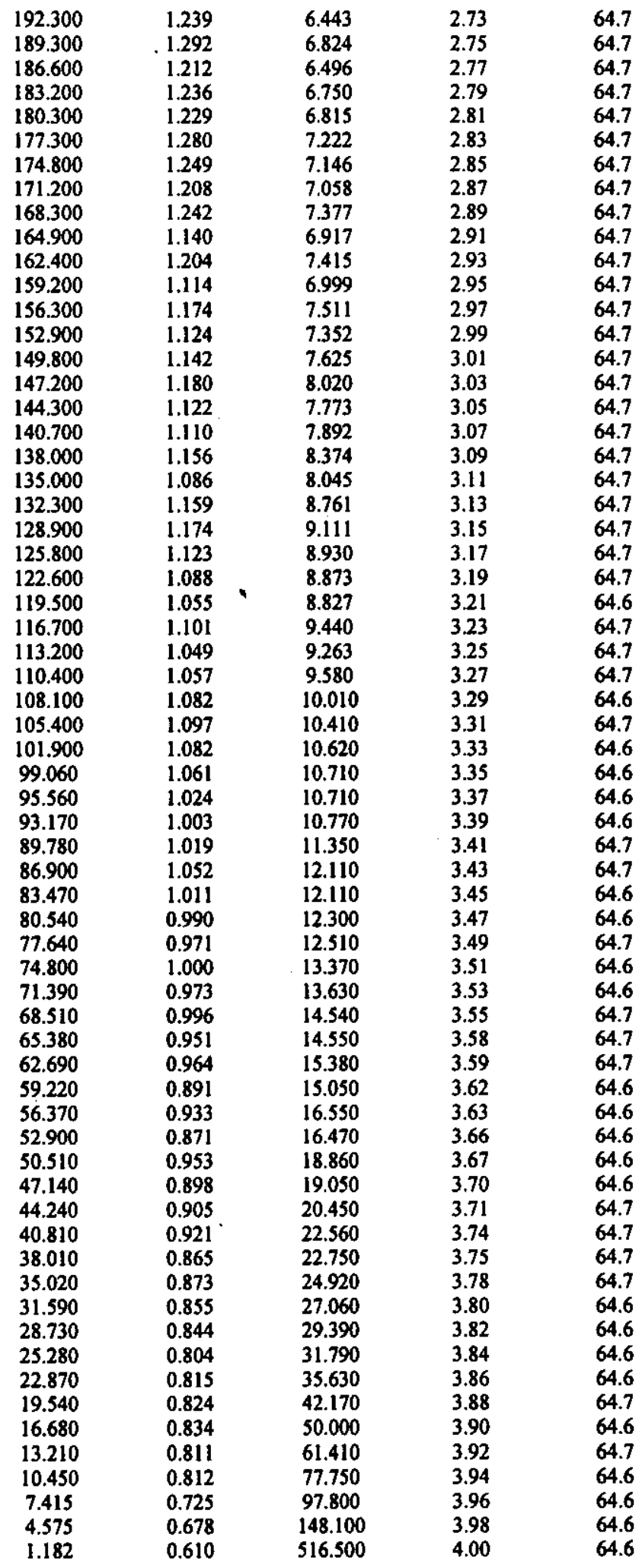


RPP-5798, Rev. 0

\section{VISCOȘITY ANALYSIS OF}

\section{HIGH SOLIDS DILUTION (300 g solids/L)}

\section{AT AMBIENT TEMPERATURE}


RPP-5798, Rev. 0

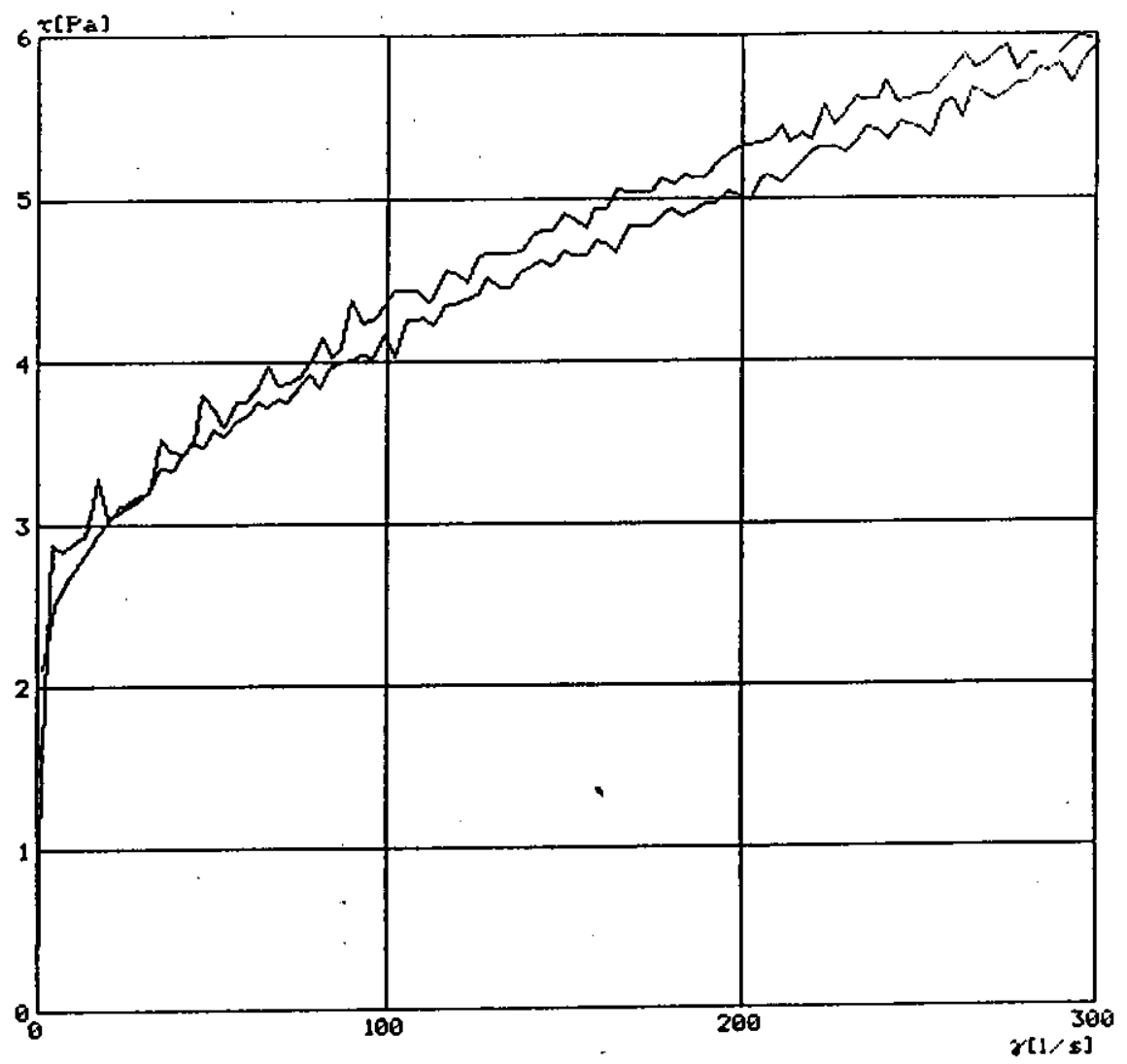

HA H K E

Operator:

cwh-gay

Substance:

clo4 306g/l

room temp

Test No.:

1

Test of:

08-30-1999

System:

M5/MV1

Temperature:

$23.0^{\circ} \mathrm{C}$

HAQKF DOAT 3 A 3

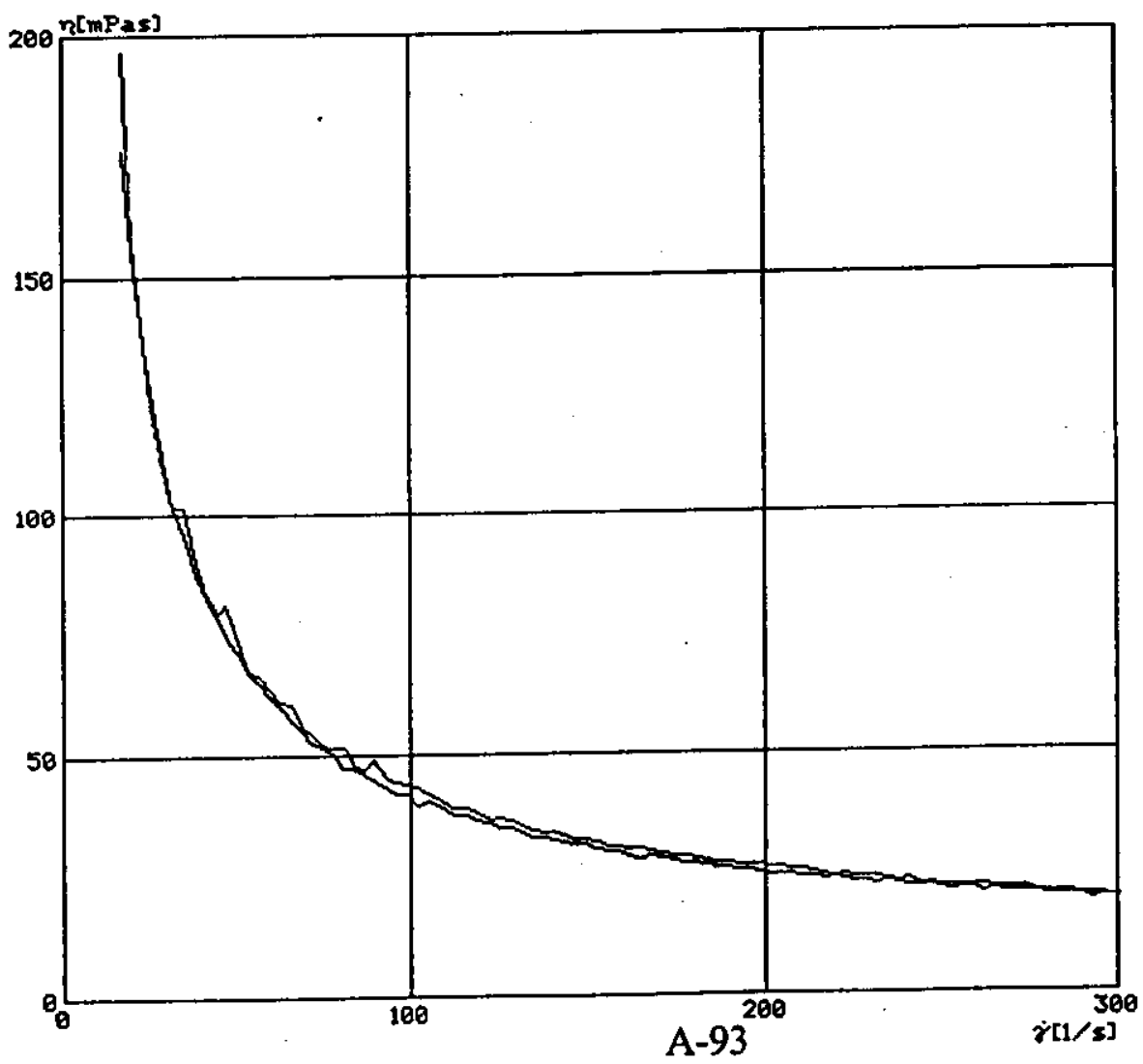

Hค $\boldsymbol{A} \mathbf{I}$

Operator:

cwh-gay

Substances

c104 300g/1

room temp

Test No.s

1

Test of:

08-30-1999

System:

M5/MV1

Temperature:

$23.0^{\circ} \mathrm{C}$

C43001.ROT 
RPP-5798, Rev. 0

\begin{tabular}{|c|c|c|c|c|}
\hline$\tau[\mathbf{P a}]$ & $\gamma\left[s^{-1}\right]$ & $\eta[\mathrm{mPas}]$ & $t \mid m i n]$ & Temp $\left.\right|^{\circ} \mathrm{C}$ \\
\hline 0.000 & 0.020 & 0.00 & 0.00 & 25.7 \\
\hline 1.449 & 1.606 & 1108.00 & 0.01 & 25.7 \\
\hline 4.060 & 2.881 & 709.70 & 0.04 & 25.7 \\
\hline 7.148 & 2.829 & 395.80 & 0.06 & 25.7 \\
\hline 10.660 & 2.886 & 270.90 & 0.08 & 25.7 \\
\hline 13.500 & 2.919 & 216.30 & 0.10 & 25.7 \\
\hline 16.680 & 3.278 & 196.50 & 0.12 & 25.7 \\
\hline 19.250 & 3.028 & 157.30 & 0.14 & 25.7 \\
\hline 22.680 & 3.066 & 135.10 & 0.16 & 25.7 \\
\hline 25.560 & 3.151 & 123.30 & 0.18 & 25.7 \\
\hline 29.050 & 3.174 & 109.30 & 0.20 & 25.7 \\
\hline 31.530 & 3.193 & 101.30 & 0.22 & 25.7 \\
\hline 34.810 & 3.529 & 101.40 & 0.24 & 25.7 \\
\hline 37.650 & 3.458 & 91.85 & 0.26 & 25.7 \\
\hline 41.060 & 3.429 & 83.52 & 0.28 & 25.7 \\
\hline 44.430 & 3.529 & 79.42 & 0.30 & 25.7 \\
\hline 46.930 & 3.803 & 81.03 & 0.32 & 25.7 \\
\hline 50.360 & 3.708 & 73.63 & 0.34 & 25.7 \\
\hline 53.160 & 3.609 & 67.88 & 0.36 & 25.8 \\
\hline 56.630 & 3.746 & 66.15 & 0.38 & 25.8 \\
\hline 59.110 & 3.755 & 63.53 & 0.40 & 25.7 \\
\hline 62.410 & 3.822 & 61.24 & 0.42 & 25.8 \\
\hline 65.320 & 3.968 & 60.74 & 0.44 & 25.8 \\
\hline 68.760 & 3.864 & 56.20 & 0.46 & 25.7 \\
\hline 71.630 & 3.878 & 54.14 & 0.48 & 25.7 \\
\hline 74.590 & 3.897 & 52.25 & 0.50 & 25.7 \\
\hline 77.410 & 3.992 & 51.57 & 0.52 & 25.7 \\
\hline 80.840 & 4.148 & 51.31 & 0.54 & 25.8 \\
\hline 83.720 & 4.025 & 48.07 & 0.56 & 25.8 \\
\hline 86.960 & 4.081 & 46.93 & 0.59 & 25.7 \\
\hline 89.550 & 4.370 & 48.79 & 0.60 & 25.7 \\
\hline 92.980 & 4.237 & 45.57 & 0.63 & 25.8 \\
\hline 95.840 & 4.256 & 44.41 & 0.64 & 25.8 \\
\hline 99.330 & 4.346 & 43.75 & 0.67 & 25.8 \\
\hline 101.800 & 4.426 & 43.49 & 0.68 & 25.8 \\
\hline 105.100 & 4.431 & 42.17 & 0.71 & 25.8 \\
\hline 108.000 & 4.431 & 41.03 & 0.72 & 25.8 \\
\hline 111.500 & 4.351 & 39.02 & 0.75 & 25.8 \\
\hline 113.800 & 4.412 & 38.77 & 0.76 & 25.8 \\
\hline 116.700 & 4.554 & 39.04 & 0.79 & 25.8 \\
\hline 119.500 & 4.535 & 37.94 & 0.80 & 25.8 \\
\hline 122.900 & 4.478 & 36.42 & 0.83 & 25.8 \\
\hline 125.800 & 4.625 & 36.76 & 0.84 & 25.8 \\
\hline 128.700 & 4.667 & 36.27 & 0.87 & 25.8 \\
\hline 132.100 & 4.653 & 35.22 & 0.89 & 25.8 \\
\hline 135.000 & 4.653 & 34.48 & 0.91 & 25.8 \\
\hline 138.400 & 4.672 & 33.76 & 0.93 & 25.8 \\
\hline 141.200 & 4.771 & 33.78 & 0.95 & 25.8 \\
\hline 144.100 & 4.790 & 33.24 & 0.97 & 25.8 \\
\hline 147.000 & 4.790 & 32.59 & 0.99 & 25.8 \\
\hline 150.400 & 4.899 & 32.57 & 1.01 & 25.8 \\
\hline 153.300 & 4.875 & 31.81 & 1.03 & 25.8 \\
\hline 156.500 & 4.818 & 30.79 & 1.05 & 25.8 \\
\hline 159.000 & 4.927 & 30.99 & 1.07 & 25.8 \\
\hline 162.400 & 4.932 & 30.37 & 1.09 & 25.8 \\
\hline 165.300 & 5.050 & 30.56 & 1.11 & 25.8 \\
\hline 168.700 & 5.036 & 29.85 & 1.13 & 25.8 \\
\hline 171.000 & 5.041 & 29.48 & 1.15 & 25.8 \\
\hline 174.400 & 5.045 & 28.93 & 1.17 & 25.8 \\
\hline 177.300 & 5.126 & 28.91 & 1.19 & 25.8 \\
\hline 180.700 & 5.088 & 28.16 & 1.21 & 25.8 \\
\hline 183.600 & 5.144 & 28.03 & 1.23 & 25.8 \\
\hline 186.400 & 5.130 & 27.52 & 1.25 & 25.8 \\
\hline 189.300 & 5.130 & 27.10 & 1.27 & 25.8 \\
\hline 192.700 & 5.215 & 27.06 & 1.29 & 25.8 \\
\hline 195.600 & 5.267 & 26.93 & 1.31 & 25.8 \\
\hline
\end{tabular}


RPP-5798, Rev. 0

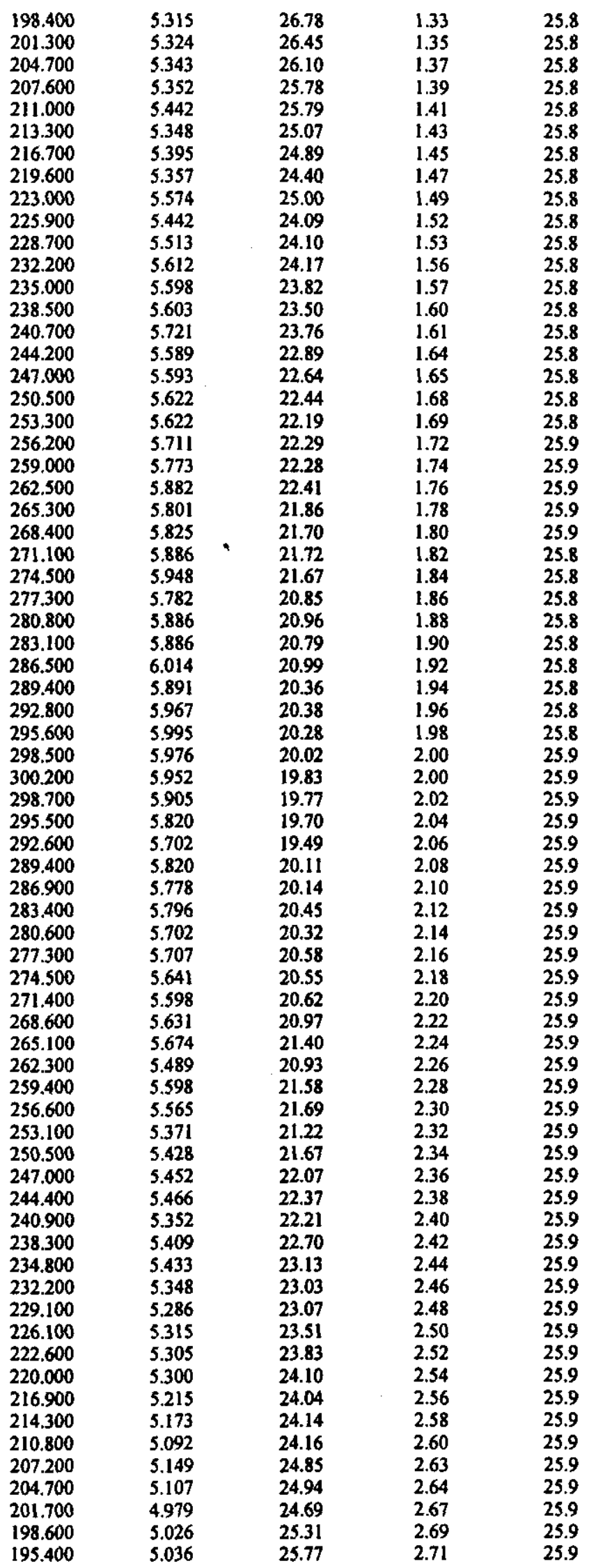


RPP-5798, Rev. 0

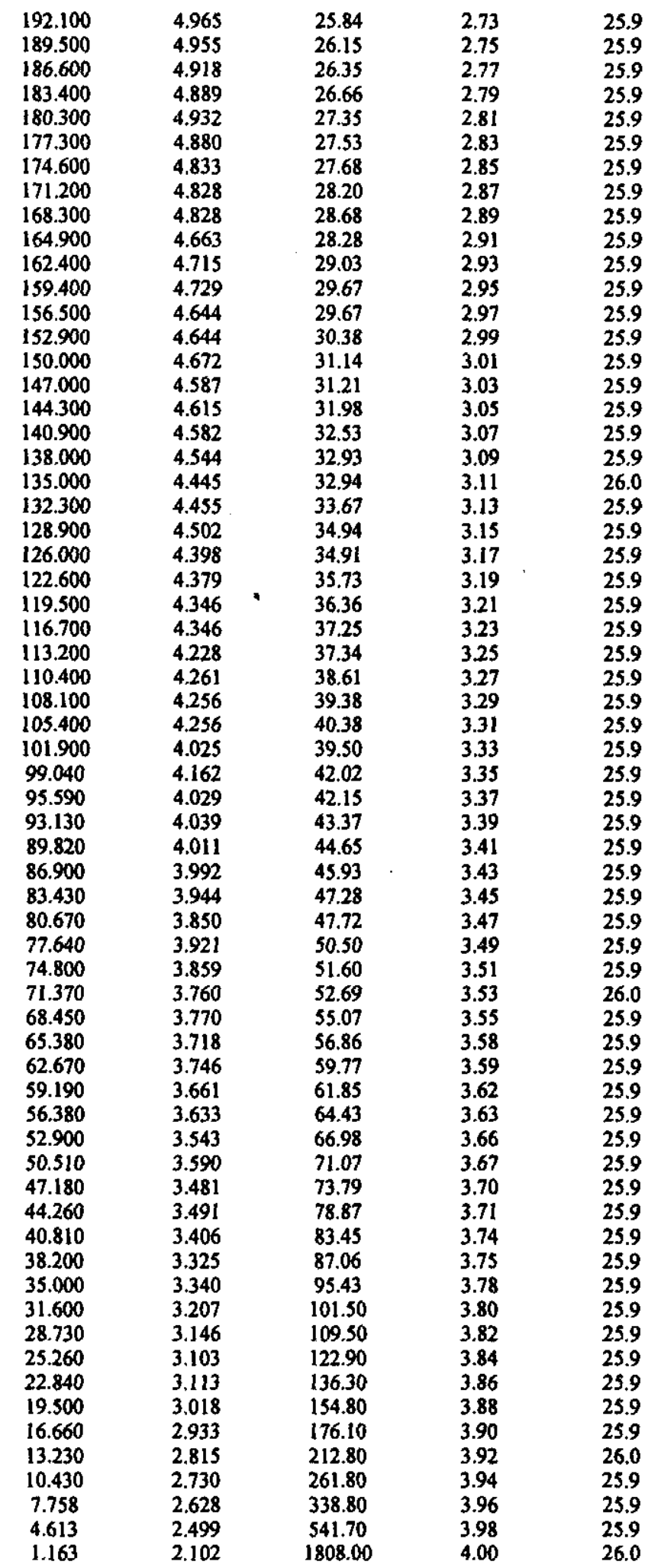


RPP-5798, Rev. 0

\section{VISCOSITY ANALYSIS OF}

\section{HIGH SOLIDS DILUTION (300 g solids/L) AT $45^{\circ} \mathrm{C}$}


RPP-5798, Rev. 0

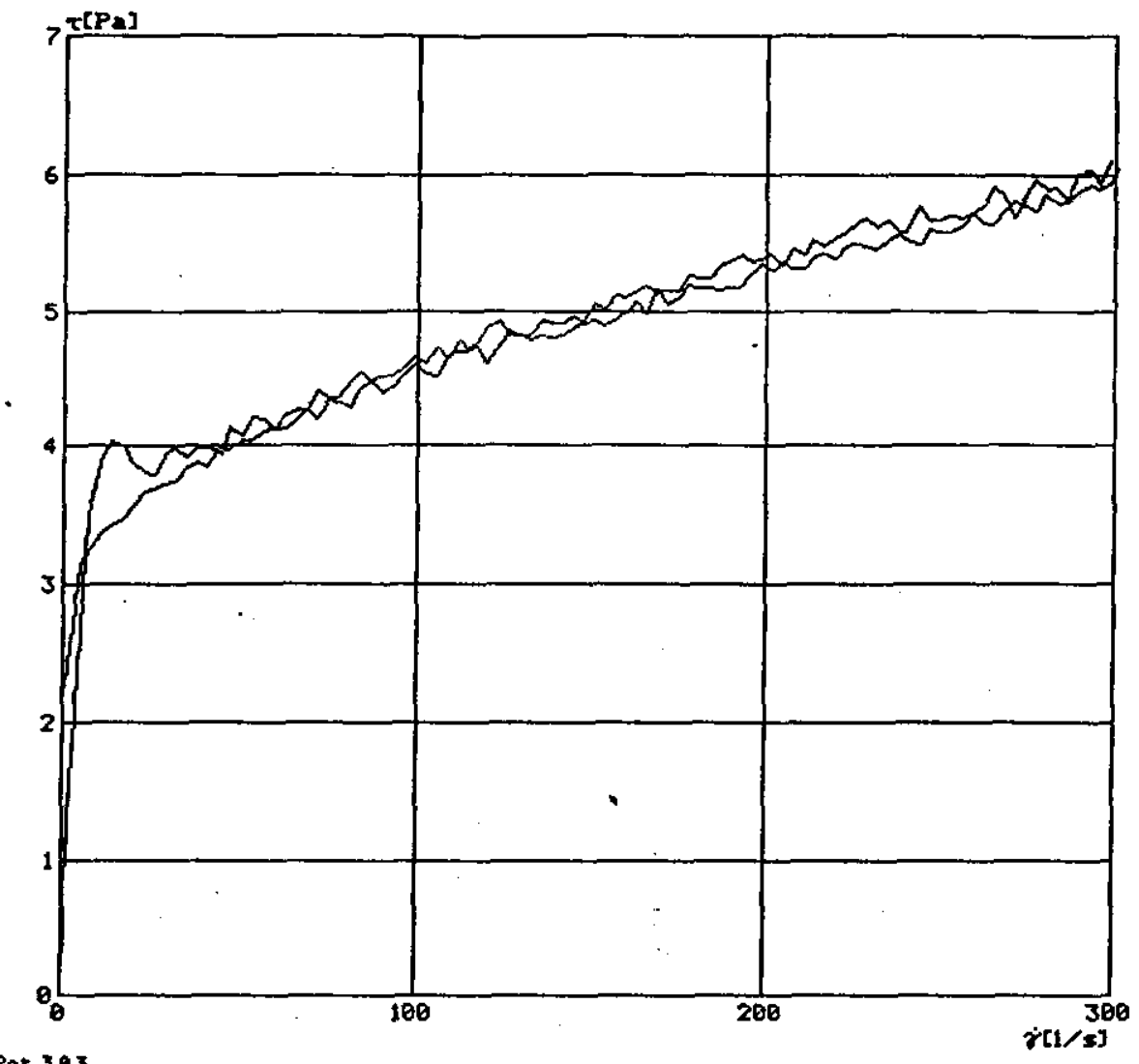

म ค ค $\mathrm{K} \mathbf{E}$

Operator:

owh-gay

Substance:

$01043009 / 1$

45c

Test No:

1

Test of:

08-30-1999

System:

M5/AV1

Temperature:

$23.0^{\circ} \mathrm{C}$

-DC C43002.ROT

HAAKE Ro: 3.0 .3

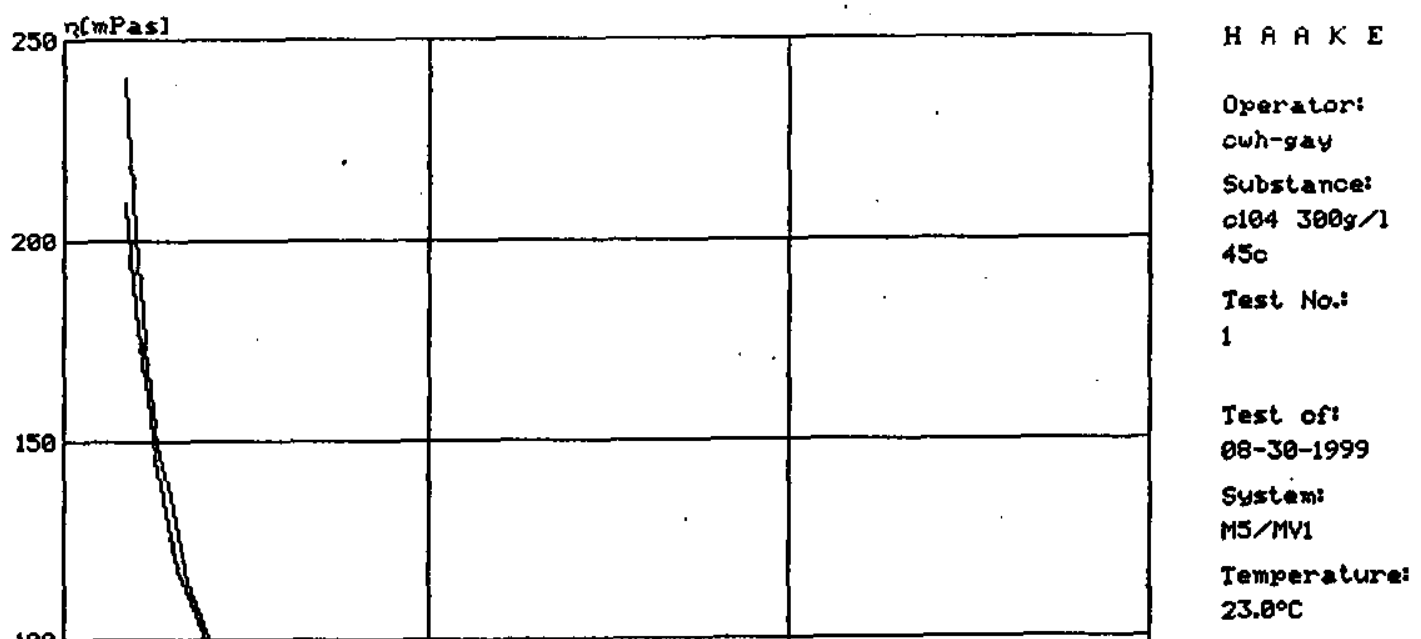

LAgKF Rnt 3 a

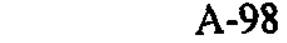

C43082.ROT 
RPP-5798, Rev. 0

\begin{tabular}{|c|c|c|c|c|}
\hline$\tau[\mathbf{P a}]$ & $\gamma\left[s^{-1}\right]$ & $\eta[m$ Pas] & t[min] & $\operatorname{Templ}^{\circ} \mathrm{Cl}$ \\
\hline 0.000 & 0.000 & 0.00 & 0.00 & 44.8 \\
\hline 1.391 & 1.253 & 900.70 & 0.02 & 44.8 \\
\hline 5.204 & 2.943 & 565.60 & 0.04 & 44.9 \\
\hline 7.091 & 3.501 & 493.70 & 0.06 & 44.8 \\
\hline 10.660 & 3.888 & 364.90 & 0.08 & 44.8 \\
\hline 13.530 & 4.039 & 298.50 & 0.10 & 44.8 \\
\hline 16.700 & 4.016 & 240.50 & 0.12 & 44.8 \\
\hline 19.290 & 3.893 & 201.80 & 0.14 & 44.8 \\
\hline 22.740 & 3.813 & 167.70 & 0.16 & 44.9 \\
\hline 25.580 & 3.803 & 148.70 & 0.18 & 44.8 \\
\hline 28.990 & 3.950 & 136.20 & 0.20 & 44.8 \\
\hline 31.550 & 3.992 & 126.50 & 0.22 & 44.9 \\
\hline 34.830 & 3.912 & 112.30 & 0.24 & 44.9 \\
\hline 37.630 & 3.992 & 106.10 & 0.26 & 44.9 \\
\hline 41.120 & 3.987 & 96.98 & 0.28 & 44.9 \\
\hline 44.430 & 3.945 & 88.79 & 0.30 & 44.9 \\
\hline 46.930 & 4.153 & 88.49 & 0.32 & 44.9 \\
\hline 50.340 & 4.082 & 81.09 & 0.34 & 44.9 \\
\hline 53.200 & 4.214 & 79.21 & 0.36 & 44.8 \\
\hline 56.670 & 4.200 & 74.11 & 0.38 & 44.9 \\
\hline 59.150 & 4.134 & 69.89 & 0.40 & 44.9 \\
\hline 62.430 & 4.219 & 67.58 & 0.42 & 44.9 \\
\hline 65.320 & 4.280 & 65.53 & 0.44 & 44.9 \\
\hline 68.790 & 4.271 & 62.08 & 0.46 & 44.9 \\
\hline 71.670 & 4.422 & 61.70 & 0.48 & 44.9 \\
\hline 74.650 & 4.365 & 58.48 & 0.50 & 44.9 \\
\hline 77.410 & 4.361 & 56.33 & 0.52 & 44.8 \\
\hline 80.880 & 4.484 & 55.44 & 0.54 & 44.9 \\
\hline 83.780 & 4.554 & 54.36 & 0.56 & 44.9 \\
\hline 86.960 & 4.488 & 51.61 & 0.59 & 44.9 \\
\hline 89.590 & 4.521 & 50.47 & 0.60 & 44.9 \\
\hline 92.980 & 4.512 & 48.52 & 0.63 & 44.9 \\
\hline 95.880 & 4.573 & 47.70 & 0.64 & 44.8 \\
\hline 99.310 & 4.658 & 46.91 & 0.67 & 44.9 \\
\hline 101.800 & 4.611 & 45.32 & 0.68 & 44.9 \\
\hline 105.200 & 4.729 & 44.97 & 0.71 & 44.9 \\
\hline 108.000 & 4.644 & 43.00 & 0.72 & 44.9 \\
\hline 111.500 & 4.776 & 42.83 & 0.75 & 44.9 \\
\hline 113.800 & 4.720 & 41.48 & 0.76 & 44.9 \\
\hline 116.700 & 4.762 & 40.82 & 0.79 & 44.9 \\
\hline 119.500 & 4.876 & 40.80 & 0.80 & 44.9 \\
\hline 122.900 & 4.928 & 40.08 & 0.83 & 44.9 \\
\hline 125.800 & 4.824 & 38.34 & 0.85 & 44.9 \\
\hline 128.700 & 4.824 & 37.49 & 0.87 & 44.9 \\
\hline 131.500 & 4.824 & 36.68 & 0.89 & 44.9 \\
\hline 135.000 & 4.928 & 36.51 & 0.91 & 44.9 \\
\hline 138.400 & 4.909 & 35.47 & 0.93 & 44.9 \\
\hline 141.200 & 4.899 & 34.69 & 0.95 & 44.9 \\
\hline 144.100 & 4.965 & 34.46 & 0.97 & 44.9 \\
\hline 147.000 & 4.932 & 33.56 & 0.99 & 44.9 \\
\hline 150.400 & 5.051 & 33.58 & 1.01 & 44.9 \\
\hline 153.300 & 5.017 & 32.74 & 1.03 & 44.9 \\
\hline 156.300 & 5.117 & 32.74 & 1.05 & 44.9 \\
\hline 159.000 & 5.102 & 32.10 & 1.07 & 44.9 \\
\hline 162.400 & 5.140 & 31.65 & 1.09 & 44.9 \\
\hline 165.300 & 5.183 & 31.36 & 1.11 & 44.9 \\
\hline 168.700 & 5.131 & 30.41 & 1.13 & 44.9 \\
\hline 171.000 & 5.150 & 30.12 & 1.15 & 44.9 \\
\hline 174.400 & 5.154 & 29.55 & 1.17 & 44.9 \\
\hline 177.300 & 5.268 & 29.72 & 1.19 & 44.9 \\
\hline 180.700 & 5.235 & 28.97 & 1.21 & 44.9 \\
\hline 183.600 & 5.235 & 28.52 & 1.23 & 44.9 \\
\hline 186.400 & 5.325 & 28.56 & 1.25 & 44.9 \\
\hline 189.300 & 5.372 & 28.38 & 1.27 & 44.9 \\
\hline 192.700 & 5.414 & 28.10 & 1.29 & 44.9 \\
\hline 195.600 & 5.362 & 27.42 & 1.31 & 44.9 \\
\hline
\end{tabular}


RPP-5798, Rev. 0

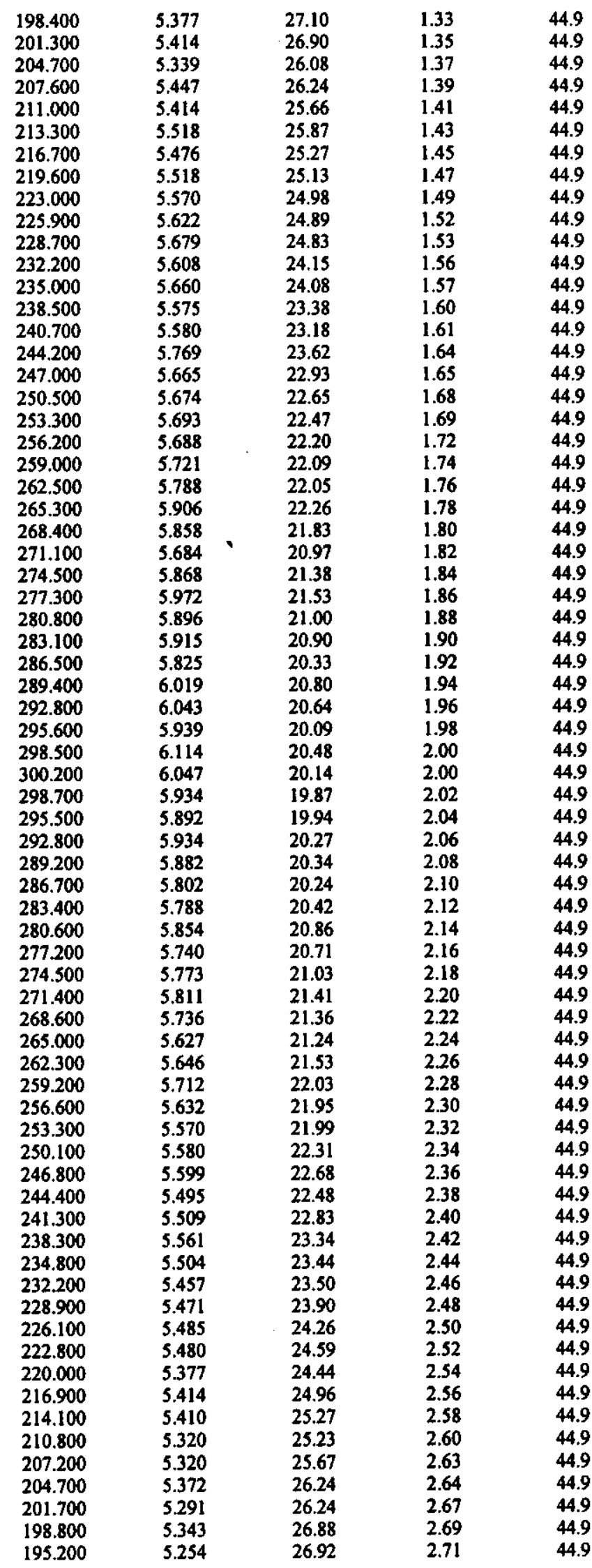


RPP-5798, Rev. 0

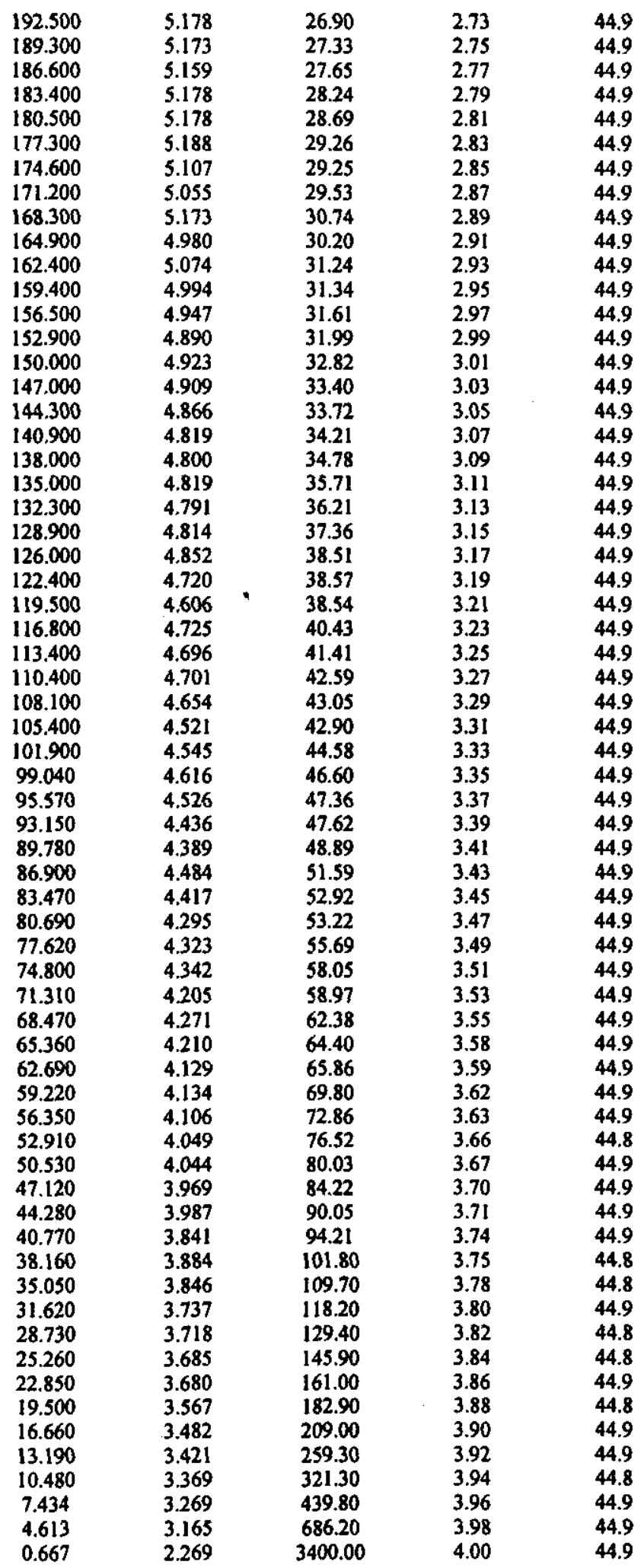


RPP-5798, Rev. 0

\section{VISCOSITY ANALYSIS OF}

HIGH SOLIDS DILUTION (300 g solids $/ \mathrm{L}$ ) AT $65^{\circ} \mathrm{C}$ 
RPP-5798, Rev. 0

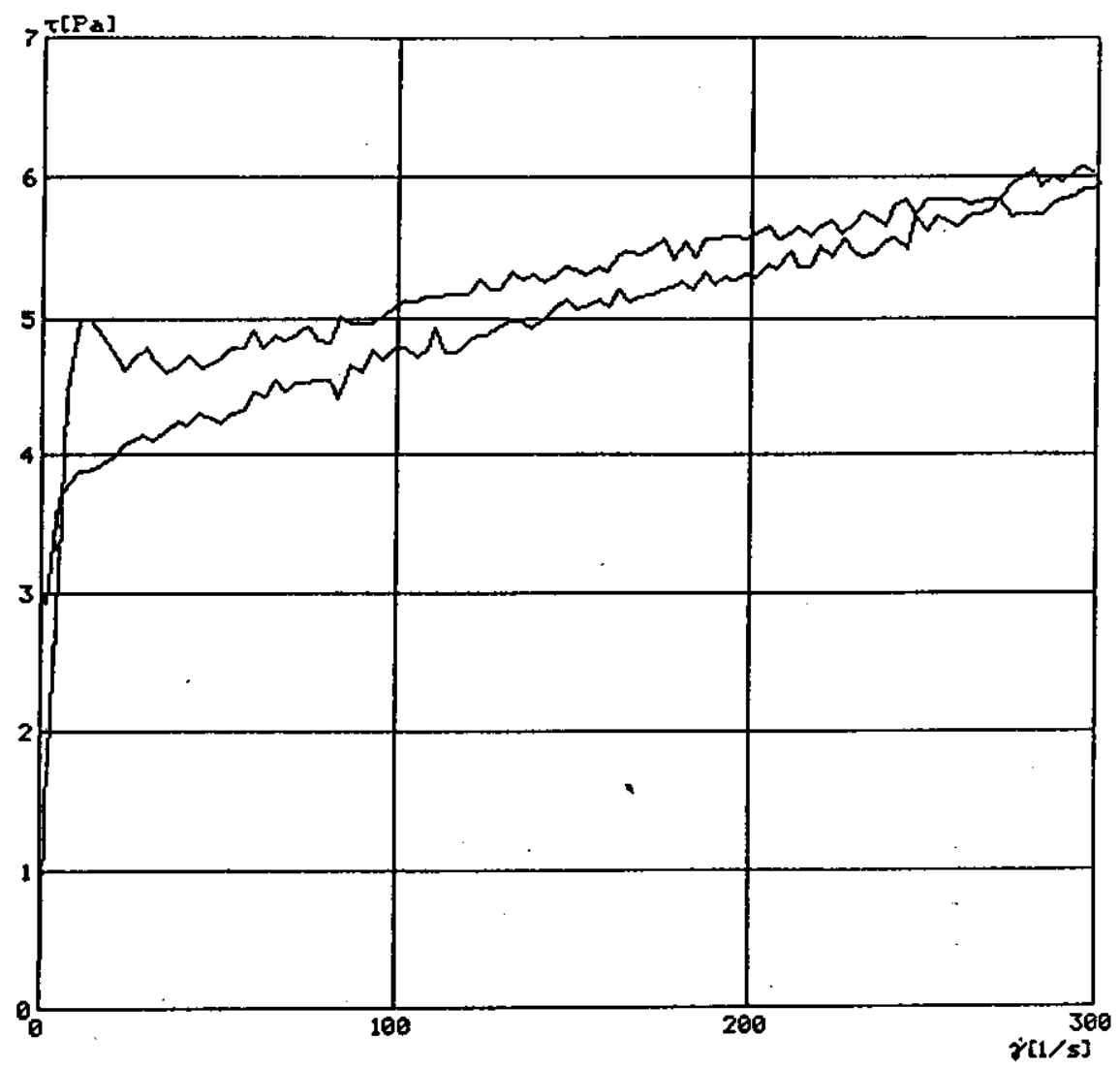

H A $A K E$

Operator:

cwh-gay

Substance:

c104 300g/1

$65 \mathrm{c}$

Test No.:

1

Test of:

88-30-1999

System:

M5/MV1

Temperature:

$23.0^{\circ} \mathrm{C}$

- C43003.ROT

MaAKF PAn YAS

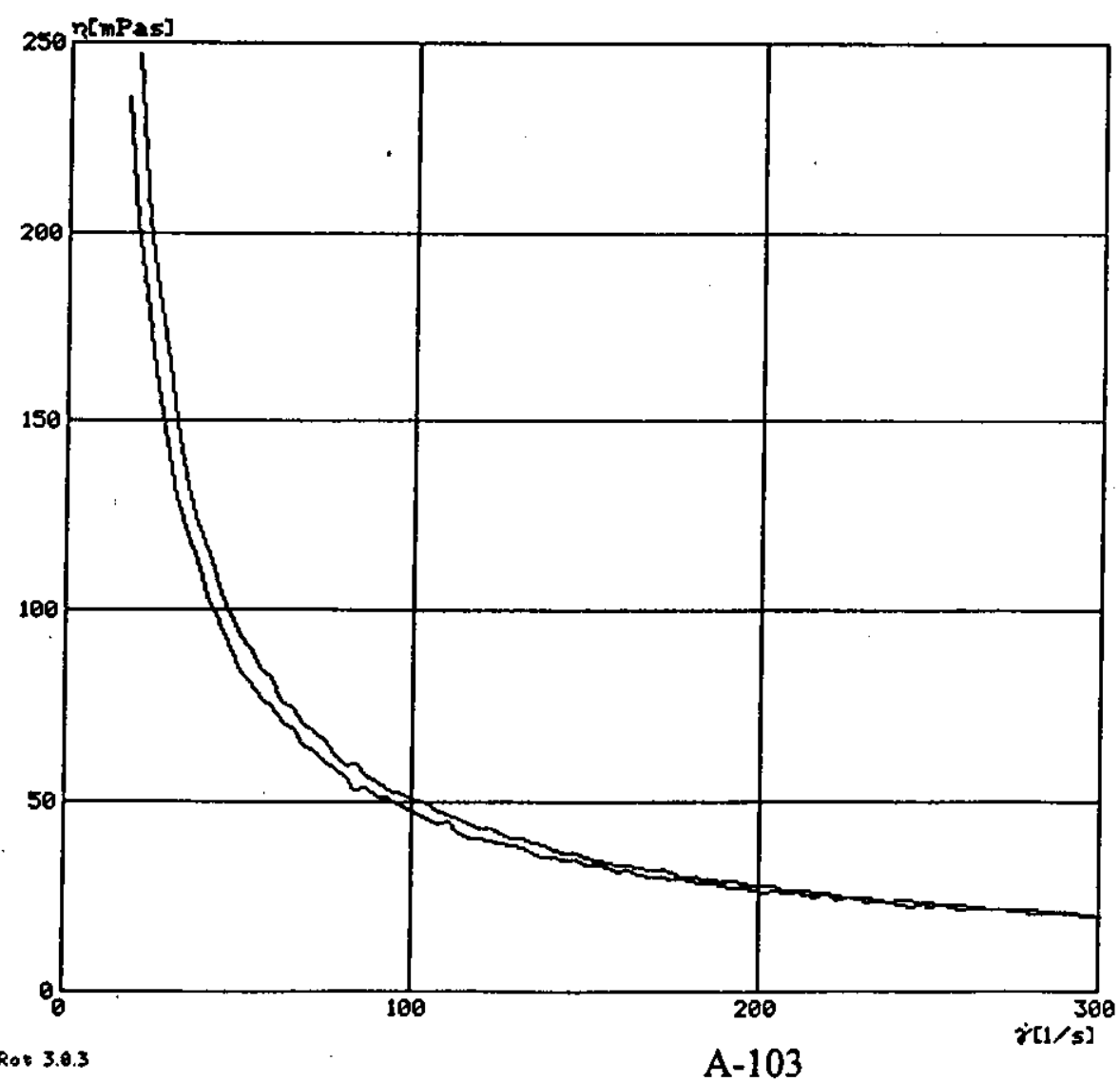

Hคค $\boldsymbol{~} \mathrm{E}$

Operator:

owh-gay

Substance:

$01043009 / 1$

$65 \mathrm{c}$

Test No.:

1

Test of:

อ8-30-1999

System:

M5/MV1

Temperature:

$23.0^{\circ} \mathrm{C}$

- C43003.Ror 
RPP-5798, Rev. 0

\begin{tabular}{|c|c|c|c|c|}
\hline$\tau[\mathbf{P}$ a $]$ & $\gamma\left[s^{-1}\right]$ & $\eta$ [mPas] & $t[m i n]$ & Temp $\left.\mid{ }^{\circ} \mathrm{C}\right]$ \\
\hline 0.000 & 0.006 & 0.00 & 0.00 & 64.4 \\
\hline 1.372 & 1.437 & 1047.00 & 0.02 & 64.4 \\
\hline 4.632 & 3.156 & 681.30 & 0.03 & 64.5 \\
\hline 7.148 & 4.450 & 622.60 & 0.06 & 64.5 \\
\hline 10.670 & 4.998 & 468.30 & 0.08 & 64.5 \\
\hline 13.530 & 4.998 & 369.30 & 0.10 & 64.5 \\
\hline 16.680 & 4.866 & 291.80 & 0.12 & 64.5 \\
\hline 19.290 & 4.767 & 247.10 & 0.14 & 64.5 \\
\hline 22.680 & 4.620 & 203.70 & 0.16 & 64.5 \\
\hline 25.580 & 4.715 & 184.30 & 0.18 & 64.5 \\
\hline 29.050 & 4.786 & 164.70 & 0.20 & 64.5 \\
\hline 31.510 & 4.701 & 149.20 & 0.22 & 64.5 \\
\hline 34.790 & 4.611 & 132.50 & 0.24 & 64.5 \\
\hline 37.650 & 4.635 & 123.10 & 0.26 & 64.4 \\
\hline 41.120 & 4.724 & $1 \cdot 14.90$ & 0.28 & 64.5 \\
\hline 44.450 & 4.644 & 104.50 & 0.30 & 64.4 \\
\hline 46.890 & 4.668 & 99.54 & 0.32 & 64.4 \\
\hline 50.360 & 4.706 & 93.44 & 0.34 & 64.4 \\
\hline 53.180 & 4.786 & 89.99 & 0.36 & 64.4 \\
\hline 56.650 & 4.786 & 84.48 & 0.38 & 64.4 \\
\hline 59.110 & 4.899 & 82.88 & 0.40 & 64.5 \\
\hline 62.450 & 4.776 & 76.49 & 0.42 & 64.5 \\
\hline 65.320 & 4.880 & 74.71 & 0.44 & 64.5 \\
\hline 68.740 & 4.843 & 70.45 & 0.46 & 64.5 \\
\hline 71.710 & 4.894 & 68.25 & 0.48 & 64.5 \\
\hline 74.650 & 4.937 & 66.14 & 0.50 & 64.5 \\
\hline 77.450 & 4.838 & 62.47 & 0.52 & 64.5 \\
\hline 80.900 & 4.824 & 59.63 & 0.54 & 64.5 \\
\hline 83.740 & 5.022 & 59.97 & 0.56 & 64.5 \\
\hline 86.960 & 4.956 & 56.99 & 0.59 & 64.4 \\
\hline 89.530 & 4.961 & 55.41 & 0.60 & 64.5 \\
\hline 93.020 & 4.961 & 53.33 & 0.63 & 64.5 \\
\hline 95.900 & 5.022 & 52.37 & 0.64 & 64.5 \\
\hline 99.370 & 5.093 & 51.25 & 0.67 & 64.5 \\
\hline 101.700 & 5.112 & 50.25 & 0.68 & 64.5 \\
\hline 105.200 & 5.121 & 48.70 & 0.71 & 64.5 \\
\hline 108.000 & 5.150 & 47.66 & 0.72 & 64.5 \\
\hline 111.500 & 5.154 & 46.22 & 0.75 & 64.5 \\
\hline 113,800 & 5.173 & 45.46 & 0.76 & 64.5 \\
\hline 116.700 & 5.164 & 44.26 & 0.79 & 64.5 \\
\hline 119.500 & 5.169 & 43.25 & 0.80 & 64.5 \\
\hline 122.900 & 5.272 & 42.88 & 0.83 & 64.5 \\
\hline 125.800 & 5.211 & 41.42 & 0.84 & 64.5 \\
\hline 128.700 & 5.211 & 40.50 & 0.87 & 64.5 \\
\hline 132.100 & 5.339 & 40.41 & 0.89 & 64.5 \\
\hline 135.000 & 5.287 & 39.17 & 0.91 & 64.5 \\
\hline 138.400 & 5.310 & 38.37 & 0.93 & 64.5 \\
\hline 141.200 & 5.258 & 37.23 & 0.95 & 64.5 \\
\hline 144.100 & 5.291 & 36.72 & 0.97 & 64.5 \\
\hline 147.000 & 5.367 & 36.52 & 0.99 & 64.5 \\
\hline 150.400 & 5.353 & 35.59 & 1.01 & 64.5 \\
\hline 153.300 & 5.315 & 34.68 & 1.03 & 64.5 \\
\hline 156.500 & 5.362 & 34.26 & 1.05 & 64.5 \\
\hline 159.000 & 5.329 & 33.52 & 1.07 & 64.5 \\
\hline 162.400 & 5.447 & 33.54 & 1.09 & 64.5 \\
\hline 165.300 & 5.476 & 33.13 & 1.11 & 64.5 \\
\hline 168.700 & 5.461 & 32.37 & 1.13 & 64.5 \\
\hline 171.000 & 5.490 & 32.11 & 1.15 & 64.5 \\
\hline 174.400 & 5.561 & 31.88 & 1.17 & 64.5 \\
\hline 177.300 & 5.424 & 30.59 & 1.19 & 64.5 \\
\hline 180.700 & 5.542 & 30.67 & 1.21 & 64.5 \\
\hline 183.600 & 5.443 & 29.65 & 1.23 & 64.5 \\
\hline 186.400 & 5.556 & 29.80 & 1.25 & 64.5 \\
\hline 189.300 & 5.565 & 29.40 & 1.27 & 64.5 \\
\hline 192.700 & 5.580 & 28.95 & 1.29 & 64.5 \\
\hline 195.600 & 5.584 & 28.55 & 1.31 & 64.5 \\
\hline
\end{tabular}


RPP-5798, Rev. 0

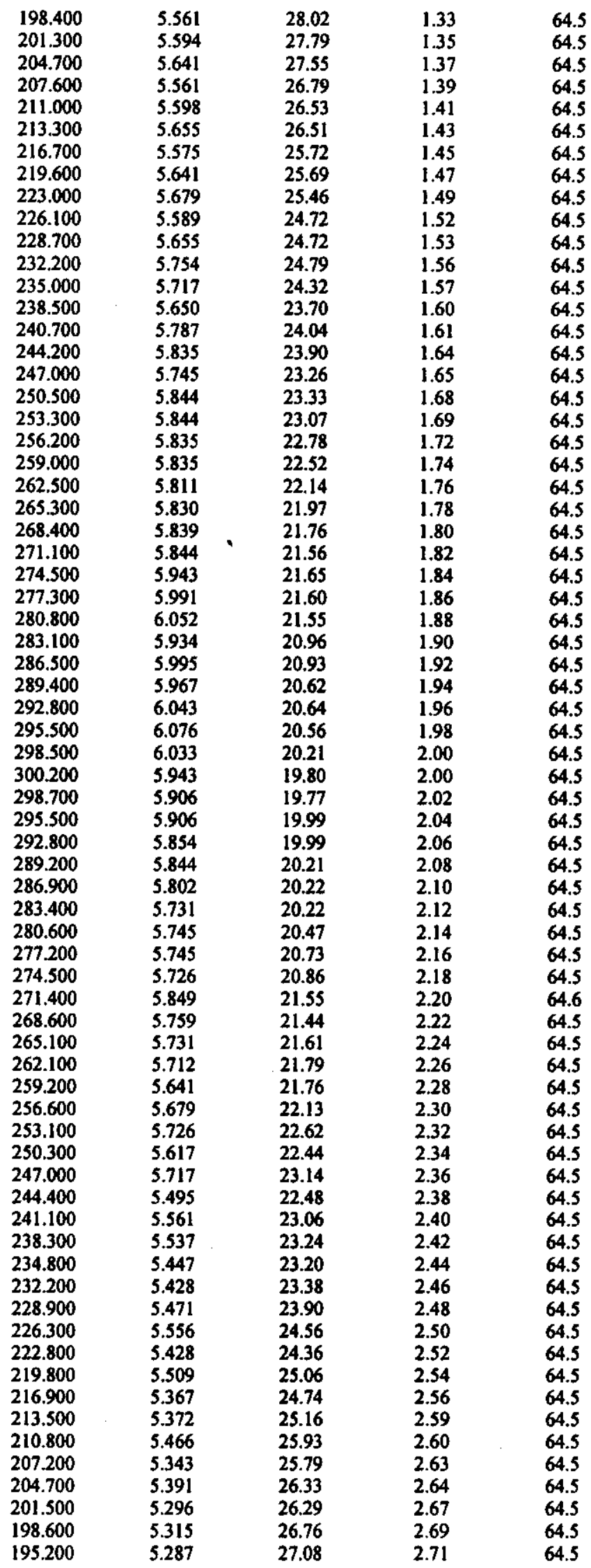


RPP-5798, Rev. 0

\begin{tabular}{|c|c|c|c|c|}
\hline 192.300 & 5.291 & 27.51 & 2.73 & 64.5 \\
\hline 189.300 & 5.244 & 27.71 & 2.75 & 64.5 \\
\hline 186.600 & 5.324 & 28.53 & 2.77 & 64.5 \\
\hline 183.400 & 5.211 & 28.42 & 2.79 & 64.5 \\
\hline 180.300 & 5.254 & 29.13 & 2.81 & 64.5 \\
\hline 177.300 & 5.230 & 29.50 & 2.83 & 64.5 \\
\hline 174.600 & 5.216 & 29.87 & 2.85 & 64.5 \\
\hline 171.200 & 5.173 & 30.22 & 2.87 & 64.5 \\
\hline 168.500 & 5.154 & 30.59 & 2.89 & 64.5 \\
\hline 165.100 & 5.126 & 31.05 & 2.91 & 64.5 \\
\hline 162.400 & 5.216 & 32.12 & 2.93 & 64.5 \\
\hline 159.200 & 5.083 & 31.94 & 2.95 & 64.5 \\
\hline 156.500 & 5.145 & 32.88 & 2.97 & 64.5 \\
\hline 152.900 & 5.083 & 33.25 & 2.99 & 64.5 \\
\hline 150.000 & 5.069 & 33.79 & 3.01 & 64.5 \\
\hline 147.200 & 5.145 & 34.96 & 3.03 & 64.5 \\
\hline 144.300 & 5.065 & 35.10 & 3.05 & 64.5 \\
\hline 140.900 & 4.989 & 35.42 & 3.07 & 64.5 \\
\hline 137.800 & 4.923 & 35.72 & 3.09 & 64.5 \\
\hline 135.000 & 4.984 & 36.93 & 3.11 & 64.5 \\
\hline 132.100 & 4.980 & 37.70 & 3.13 & 64.5 \\
\hline 128.900 & 4.942 & 38.35 & 3.15 & 64.5 \\
\hline 125.200 & 4.871 & 38.89 & 3.17 & 64.5 \\
\hline 122.400 & 4.871 & 39.80 & 3.19 & 64.5 \\
\hline 119.500 & 4.819 & 40.32 & 3.21 & 64.5 \\
\hline 116.800 & 4.748 & 40.63 & 3.23 & 64.5 \\
\hline 113.400 & 4.743 & 41.82 & 3.25 & 64.5 \\
\hline 110.400 & 4.918 & 44.56 & 3.27 & 64.5 \\
\hline 108.100 & 4.762 & 44.06 & 3.29 & 64.5 \\
\hline 105.400 & 4.715 & 44.75 & 3,31 & 64.5 \\
\hline 101.900 & 4.776 & 46.86 & 3.33 & 64.5 \\
\hline 98.990 & 4.776 & 48.25 & 3.35 & 64.5 \\
\hline 95.590 & 4.691 & 49.08 & 3.37 & 64.5 \\
\hline 93.130 & 4.772 & 51.23 & 3.39 & 64.5 \\
\hline 89.800 & 4.611 & 51.35 & 3.41 & 64.5 \\
\hline 86.940 & 4.658 & 53.58 & 3.43 & 64.5 \\
\hline 83.450 & 4.422 & 52.99 & 3.45 & 64.5 \\
\hline 80.690 & 4.559 & 56.50 & 3,47 & 64.5 \\
\hline 77.660 & 4.554 & 58.65 & 3.49 & 64.5 \\
\hline 74.840 & 4.531 & 60.54 & 3.51 & 64. \\
\hline 71.370 & 4.531 & 63.48 & 3.53 & 64.5 \\
\hline 68.450 & 4.460 & 65.15 & 3.55 & 64.5 \\
\hline 65.380 & 4.559 & 69.73 & 3.58 & 64.5 \\
\hline 62.670 & 4.431 & 70.71 & 3.59 & 64.5 \\
\hline 59.220 & 4.465 & 75.38 & 3.62 & 64.5 \\
\hline 56.350 & 4.332 & 76.89 & 3,63 & 64. \\
\hline 52.910 & 4.313 & 81.51 & 3.66 & 64. \\
\hline 50.510 & 4.238 & 83.89 & 3.67 & 64.5 \\
\hline 47.120 & 4.299 & 91.24 & 3.70 & 64. \\
\hline 44.260 & 4.309 & 97.35 & 3.71 & 64.5 \\
\hline 40.790 & 4.224 & 103.50 & 3.74 & 64.5 \\
\hline 38.220 & 4.252 & 111.30 & 3.75 & 64.5 \\
\hline 35.020 & 4.191 & 119.70 & 3.78 & 64. \\
\hline 31.600 & 4.115 & 130.20 & 3.80 & 64. \\
\hline 28.690 & 4.148 & 144.60 & 3.82 & 64. \\
\hline 25.280 & 4.110 & 162.60 & 3.84 & 64. \\
\hline 22.890 & 4.077 & 178.10 & 3.86 & 64. \\
\hline 19.540 & 3.968 & 203.10 & 3.88 & 64. \\
\hline 16.700 & 3.935 & 235.70 & 3.90 & 64. \\
\hline 13.210 & 3.893 & 294.70 & 3.92 & 64. \\
\hline 10.500 & 3.879 & 369.30 & 3.94 & 64. \\
\hline 7.434 & 3.794 & 510.30 & 3.96 & 64. \\
\hline 4.594 & 3.699 & 805.20 & 3.98 & 64. \\
\hline 1.182 & 2.929 & 2478.00 & 4.00 & 64.5 \\
\hline
\end{tabular}


RPP-5798, Rev. 0

APPENDIX B

PARTICLE SIZE DISTRIBUTION DATA

B-1 
HORIBA LA-910

Laser ecatterino particlo size distribution analyzer

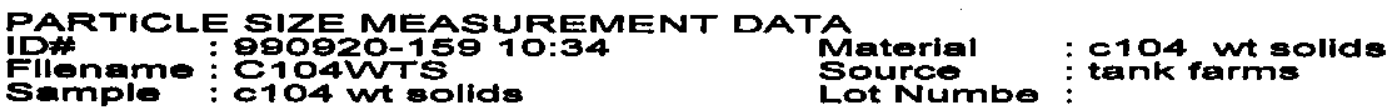

Conaltion

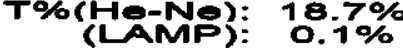

Agitation : 0 Circultn :

Rist.Form: Sharp S. Samplina times : 100

Format

Dist.base : Volume Scallng : Auto Axis : LogX-LinoarY

Date

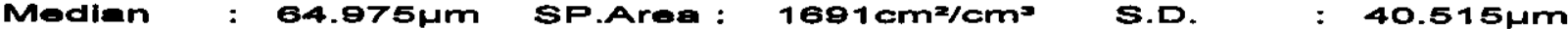

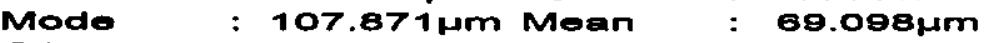

C.V. $\quad$ : $58.63 \%$

Span : (D 10.0-D 90.0)/D50 = 1.662

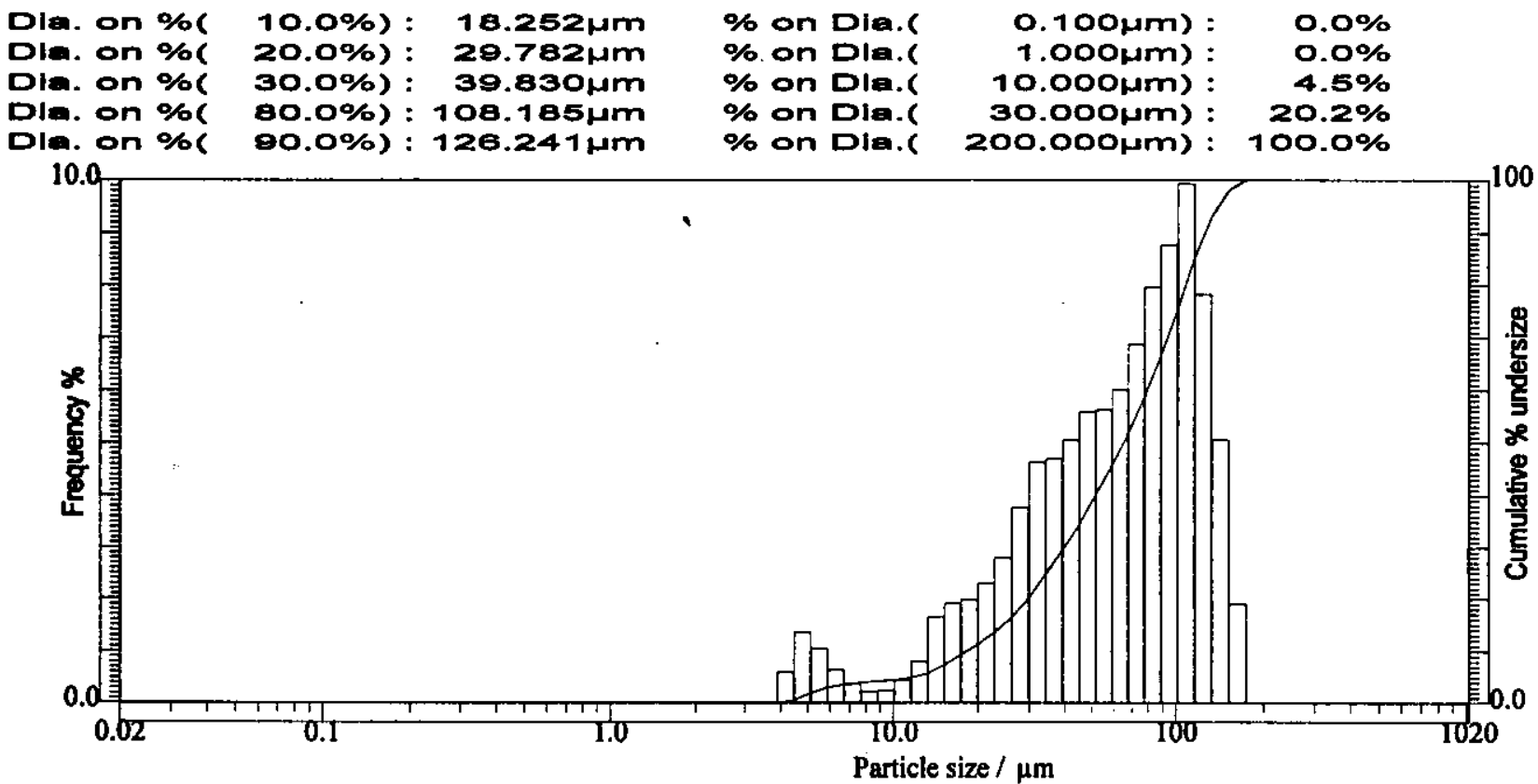

Size(رm) Froq(\%)Und(\%)

\begin{tabular}{|c|c|c|}
\hline 1019.5 & 0.00 & 100.00 \\
\hline 890.1 & 0.00 & 100.00 \\
\hline 777.1 & 0.00 & 100.00 \\
\hline 678.5 & 0.00 & 100.00 \\
\hline 892.4 & 0.00 & 100.00 \\
\hline 517.2 & 0.00 & 100.00 \\
\hline 451.6 & 0.00 & 100.00 \\
\hline 394.2 & 0.00 & 100.00 \\
\hline 344.2 & 0.00 & 100.00 \\
\hline 300.8 & 0.00 & 100.00 \\
\hline 282.4 & 0.00 & 100.00 \\
\hline 229.1 & 0.00 & 100.00 \\
\hline 200.0 & 0.00 & 100.00 \\
\hline 174.6 & 1.89 & 100.00 \\
\hline 162.5 & 5.06 & 98.11 \\
\hline 133.1 & 7.82 & 93.05 \\
\hline 110.2 & $\mathbf{9 . 9 3}$ & 85.23 \\
\hline 101.5 & 8.78 & 76.31 \\
\hline 98.58 & 7.96 & $6 \mathbf{6 3}$ \\
\hline 77.34 & 6.86 & 58.57 \\
\hline 97.62 & 0.01 & 51.70 \\
\hline 68.95 & 5.62 & 45.70 \\
\hline 51.47 & 5.59 & 40.08 \\
\hline 44.94 & 5.05 & 34.49 \\
\hline 38.23 & 4.70 & 28.44 \\
\hline 34.25 & 4.63 & 24.74 \\
\hline 28.91 & 3.76 & 20.12 \\
\hline
\end{tabular}

Sizo(Hm) Freq(\%)Und(\%)

\begin{tabular}{lll}
\hline 28.11 & 2.80 & 16.35 \\
22.80 & 2.30 & 13.56 \\
19.90 & 1.98 & 11.26 \\
17.38 & 1.90 & 9.28 \\
15.17 & 1.65 & 7.38 \\
13.25 & 0.80 & 5.73 \\
11.58 & 0.44 & 4.92 \\
10.10 & 0.25 & 4.49 \\
8.816 & 0.22 & 4.24 \\
7.697 & 0.37 & 4.01 \\
6.720 & 0.64 & 3.64 \\
5.867 & 1.04 & 3.00 \\
5.122 & 1.36 & 1.96 \\
4.472 & 0.60 & 0.60 \\
3.905 & 0.00 & 0.00 \\
3.409 & 0.00 & 0.00 \\
2.976 & 0.00 & 0.00 \\
2.599 & 0.00 & 0.00 \\
2.269 & 0.00 & 0.00 \\
1.981 & 0.00 & 0.00 \\
1.729 & 0.00 & 0.00 \\
1.510 & 0.00 & 0.00 \\
1.318 & 0.00 & 0.00 \\
1.151 & 0.00 & 0.00 \\
1.005 & 0.00 & 0.00 \\
0.877 & $\mathrm{~B}-9.00$ & 0.00 \\
0.769 & 0.00 & 0.00
\end{tabular}

Size(Hm) Freq(\%)Und(\%)

$\begin{array}{lll}0.669 & 0.00 & 0.00 \\ 0.584 & 0.00 & 0.00 \\ 0.510 & 0.00 & 0.00 \\ 0.445 & 0.00 & 0.00 \\ 0.389 & 0.00 & 0.00 \\ 0.339 & 0.00 & 0.00 \\ 0.296 & 0.00 & 0.00 \\ 0.259 & 0.00 & 0.00 \\ 0.226 & 0.00 & 0.00 \\ 0.197 & 0.00 & 0.00 \\ 0.172 & 0.00 & 0.00 \\ 0.150 & 0.00 & 0.00 \\ 0.131 & 0.00 & 0.00 \\ 0.115 & 0.00 & 0.00 \\ 0.100 & 0.00 & 0.00 \\ 0.087 & 0.00 & 0.00 \\ 0.076 & 0.00 & 0.00 \\ 0.067 & 0.00 & 0.00 \\ 0.058 & 0.00 & 0.00 \\ 0.051 & 0.00 & 0.00 \\ 0.044 & 0.00 & 0.00 \\ 0.039 & 0.00 & 0.00 \\ 0.034 & 0.00 & 0.00 \\ 0.029 & 0.00 & 0.00 \\ 0.026 & 0.00 & 0.00 \\ 0.022 & 0.00 & 0.00 \\ & & \end{array}$


HORIBA LA-910

Laser scattering particle slze distribution analyzer

PARTICLE SIZE MEASUREMENT DATA

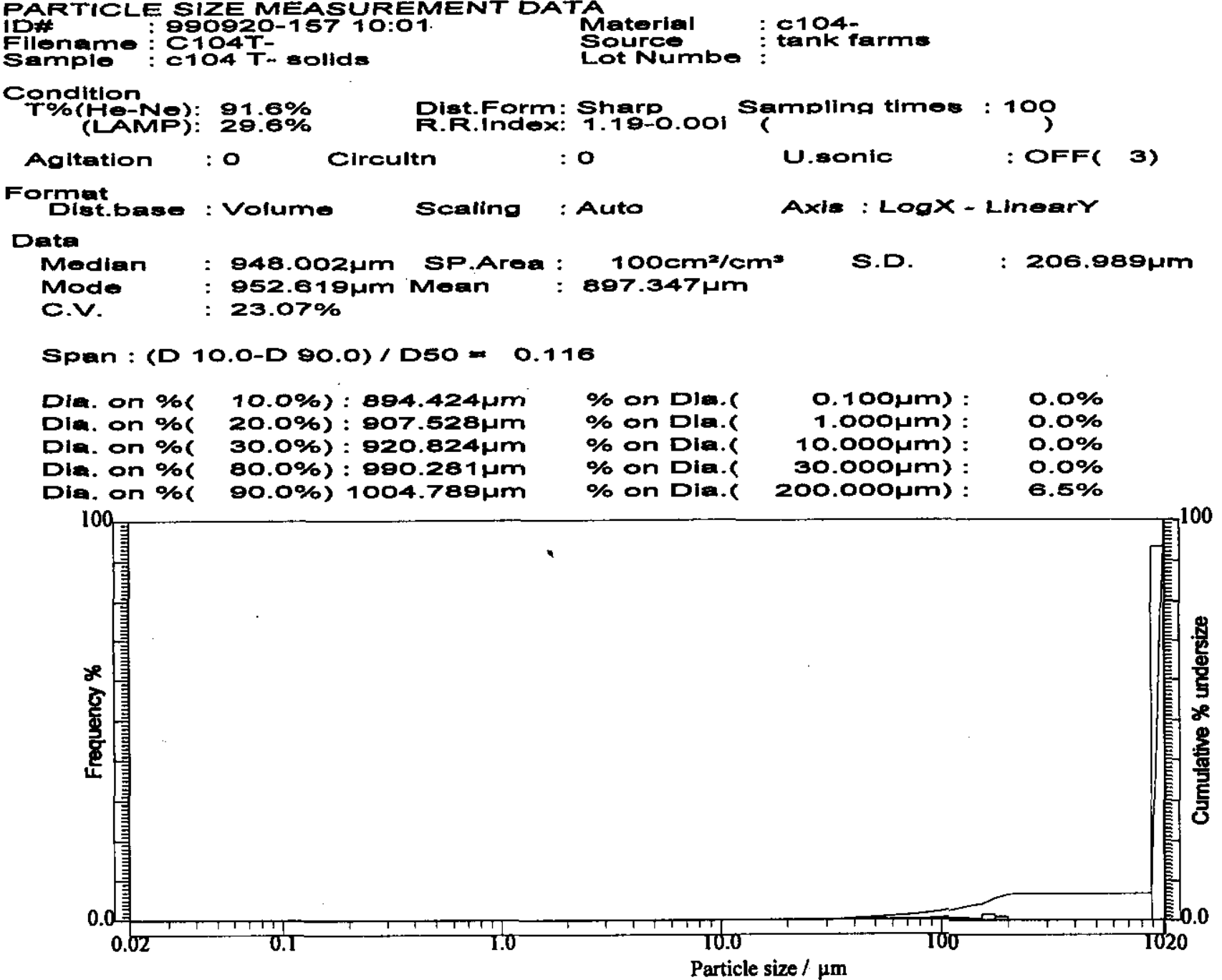

Size(pm) Freq(\%)Und(\%)

\begin{tabular}{rrr}
\hline 1019.5 & 93.32 & 100.00 \\
890.1 & 0.00 & 6.68 \\
777.1 & 0.00 & 6.68 \\
678.5 & 0.00 & 6.68 \\
582.4 & 0.00 & 6.68 \\
517.2 & 0.00 & 6.68 \\
451.6 & 0.00 & 6.68 \\
394.2 & 0.00 & 6.68 \\
344.2 & 0.00 & 6.68 \\
300.5 & 0.00 & 6.68 \\
262.4 & 0.00 & 6.68 \\
228.1 & 0.22 & 6.68 \\
200.0 & 0.95 & 6.46 \\
174.6 & 1.49 & 5.51 \\
182.5 & 0.48 & 4.02 \\
133.1 & 0.73 & 3.54 \\
116.2 & 0.68 & 2.81 \\
101.5 & 0.41 & 2.15 \\
88.58 & 0.33 & 1.74 \\
77.34 & 0.26 & 1.41 \\
97.52 & 0.22 & 1.15 \\
68.95 & 0.19 & 0.93 \\
81.47 & 0.18 & 0.75 \\
44.94 & 0.18 & 0.57 \\
38.23 & 0.20 & 0.40 \\
34.25 & 0.20 & 0.20 \\
29.91 & 0.00 & 0.00
\end{tabular}

Size( $\mu m)$ Freq(\%)Und(\%)

\begin{tabular}{lll}
\hline 28.11 & 0.00 & 0.00 \\
22.80 & 0.00 & 0.00 \\
19.90 & 0.00 & 0.00 \\
17.38 & 0.00 & 0.00 \\
15.17 & 0.00 & 0.00 \\
13.25 & 0.00 & 0.00 \\
11.56 & 0.00 & 0.00 \\
10.10 & 0.00 & 0.00 \\
8.816 & 0.00 & 0.00 \\
7.697 & 0.00 & 0.00 \\
6.720 & 0.00 & 0.00 \\
5.867 & 0.00 & 0.00 \\
5.122 & 0.00 & 0.00 \\
4.472 & 0.00 & 0.00 \\
3.905 & 0.00 & 0.00 \\
3.409 & 0.00 & 0.00 \\
2.976 & 0.00 & 0.00 \\
2.599 & 0.00 & 0.00 \\
2.269 & 0.00 & 0.00 \\
1.981 & 0.00 & 0.00 \\
1.729 & 0.00 & 0.00 \\
1.510 & 0.00 & 0.00 \\
1.318 & 0.00 & 0.00 \\
1.151 & 0.00 & 0.00 \\
1.005 & 0.00 & 0.00 \\
0.877 & $\mathrm{~B}-9.00$ & 0.00 \\
0.766 & 0.00 & 0.00
\end{tabular}

Size(Hm) Freq(\%)Und(\%)

\begin{tabular}{lll}
\hline 0.698 & 0.00 & 0.00 \\
0.594 & 0.00 & 0.00 \\
0.510 & 0.00 & 0.00 \\
0.446 & 0.00 & 0.00 \\
0.389 & 0.00 & 0.00 \\
0.359 & 0.00 & 0.00 \\
0.296 & 0.00 & 0.00 \\
0.259 & 0.00 & 0.00 \\
0.226 & 0.00 & 0.00 \\
0.197 & 0.00 & 0.00 \\
0.172 & 0.00 & 0.00 \\
0.150 & 0.00 & 0.00 \\
0.131 & 0.00 & 0.00 \\
0.115 & 0.00 & 0.00 \\
0.100 & 0.00 & 0.00 \\
0.087 & 0.00 & 0.00 \\
0.076 & 0.00 & 0.00 \\
0.067 & 0.00 & 0.00 \\
0.058 & 0.00 & 0.00 \\
0.0851 & 0.00 & 0.00 \\
0.044 & 0.00 & 0.00 \\
0.039 & 0.00 & 0.00 \\
0.034 & 0.00 & 0.00 \\
0.029 & 0.00 & 0.00 \\
0.028 & 0.00 & 0.00 \\
0.022 & 0.00 & 0.00 \\
& &
\end{tabular}


Laser scattering particle size distribution analyzor

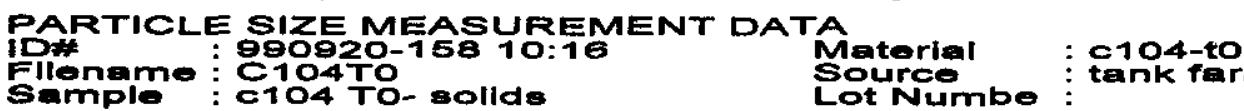

Span : (D 10.0-D 90.0)/D50 = 1.168

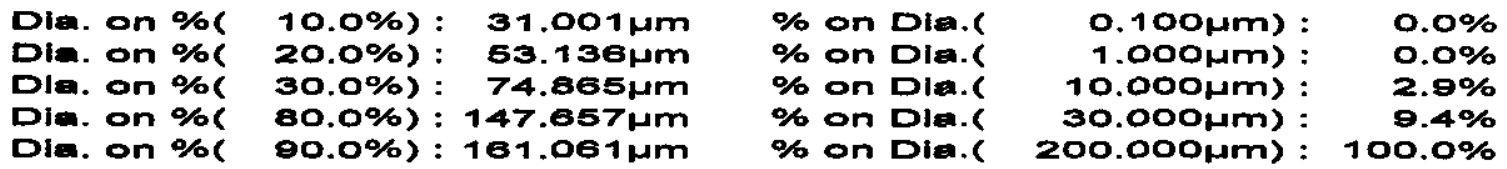

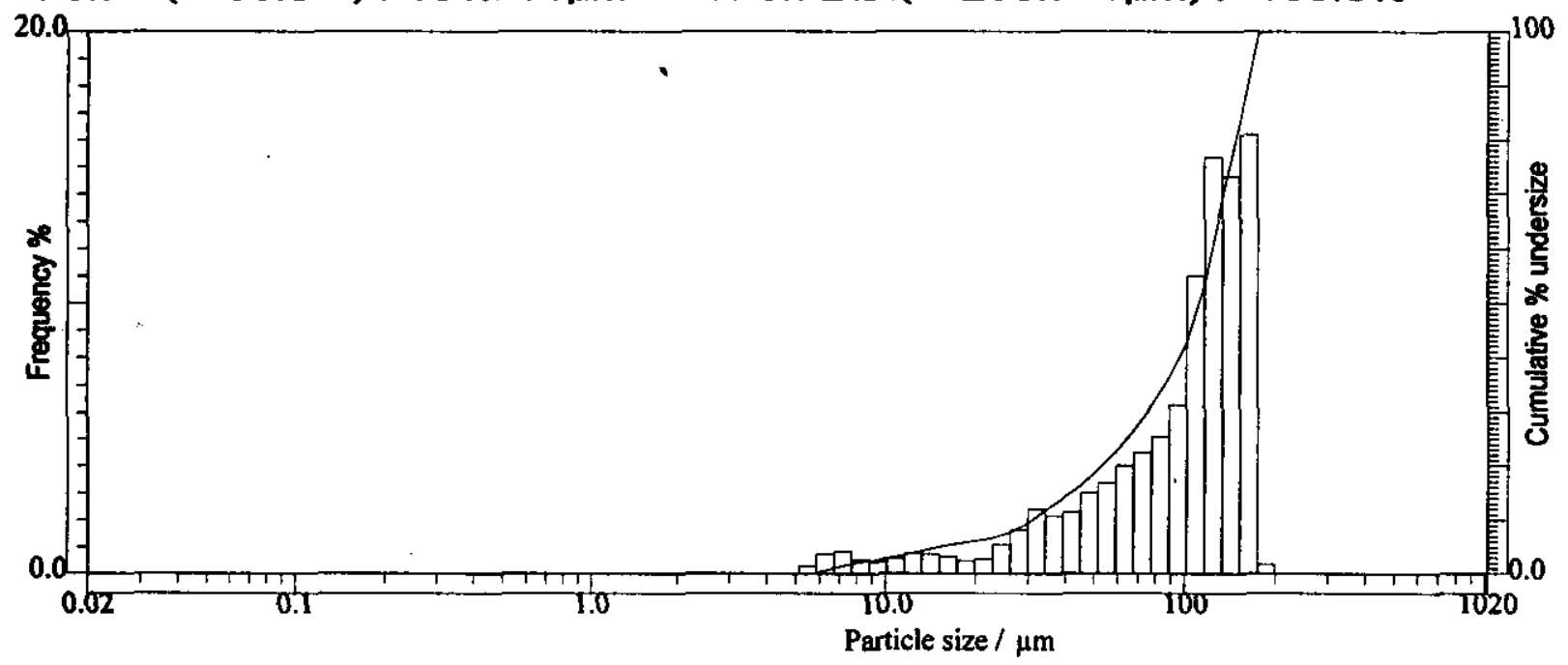

Size(Hm) Freq(\%)Und(\%)

\begin{tabular}{rrr}
\hline 1019.5 & 0.00 & 100.00 \\
890.1 & 0.00 & 100.00 \\
777.1 & 0.00 & 100.00 \\
678.5 & 0.00 & 100.00 \\
682.4 & 0.00 & 100.00 \\
617.2 & 0.00 & 100.00 \\
481.8 & 0.00 & 100.00 \\
384.2 & 0.00 & 100.00 \\
344.2 & 0.00 & 100.00 \\
300.5 & 0.00 & 100.00 \\
282.4 & 0.00 & 100.00 \\
229.1 & 0.00 & 100.00 \\
200.0 & 0.36 & 100.00 \\
174.6 & 16.20 & 99.64 \\
162.5 & 14.63 & 83.45 \\
133.1 & 15.37 & 68.81 \\
118.2 & 10.99 & 63.44 \\
101.5 & 6.27 & 42.45 \\
88.68 & 5.10 & 38.18 \\
77.34 & 4.49 & 31.08 \\
67.82 & 4.01 & 26.58 \\
88.85 & 3.37 & 22.58 \\
61.47 & 2.99 & 19.21 \\
44.94 & 2.29 & 16.22 \\
39.23 & 2.17 & 13.93 \\
34.25 & 2.40 & 11.77 \\
29.91 & 1.63 & 9.36
\end{tabular}

Size( $(\mu m)$ Freq(\%)Und(\%)

\begin{tabular}{lll}
\hline 26.11 & 1.09 & 7.73 \\
22.80 & 0.56 & 6.64 \\
19.90 & 0.48 & 6.08 \\
17.38 & 0.65 & 5.60 \\
15.17 & 0.74 & 4.95 \\
13.25 & 0.77 & 4.22 \\
11.56 & 0.56 & 3.45 \\
10.10 & 0.45 & 2.89 \\
8.816 & 0.54 & 2.44 \\
7.697 & 0.85 & 1.90 \\
6.720 & 0.76 & 1.06 \\
5.867 & 0.30 & 0.30 \\
5.122 & 0.00 & 0.00 \\
4.472 & 0.00 & 0.00 \\
3.805 & 0.00 & 0.00 \\
3.409 & 0.00 & 0.00 \\
2.976 & 0.00 & 0.00 \\
2.699 & 0.00 & 0.00 \\
2.269 & 0.00 & 0.00 \\
1.981 & 0.00 & 0.00 \\
1.729 & 0.00 & 0.00 \\
1.510 & 0.00 & 0.00 \\
1.318 & 0.00 & 0.00 \\
1.151 & 0.00 & 0.00 \\
1.095 & 0.00 & 0.00 \\
0.877 & $B-9.00$ & 0.00 \\
0.766 & 0.00 & 0.00
\end{tabular}

Sizo(1m) Froq(\%)Und(\%)

\begin{tabular}{lll}
\hline 0.669 & 0.00 & 0.00 \\
0.584 & 0.00 & 0.00 \\
0.510 & 0.00 & 0.00 \\
0.445 & 0.00 & 0.00 \\
0.389 & 0.00 & 0.00 \\
0.339 & 0.00 & 0.00 \\
0.296 & 0.00 & 0.00 \\
0.259 & 0.00 & 0.00 \\
0.226 & 0.00 & 0.00 \\
0.197 & 0.00 & 0.00 \\
0.172 & 0.00 & 0.00 \\
0.150 & 0.00 & 0.00 \\
0.131 & 0.00 & 0.00 \\
0.115 & 0.00 & 0.00 \\
0.100 & 0.00 & 0.00 \\
0.087 & 0.00 & 0.00 \\
0.078 & 0.00 & 0.00 \\
0.087 & 0.00 & 0.00 \\
0.058 & 0.00 & 0.00 \\
0.051 & 0.00 & 0.00 \\
0.044 & 0.00 & 0.00 \\
0.038 & 0.00 & 0.00 \\
0.034 & 0.00 & 0.00 \\
0.029 & 0.00 & 0.00 \\
0.028 & 0.00 & 0.00 \\
0.022 & 0.00 & 0.00 \\
& &
\end{tabular}


HORIBA LA-910

Lasor acatterino particlo size distribution analyzer

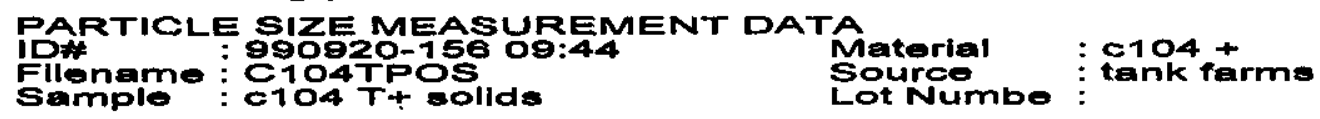

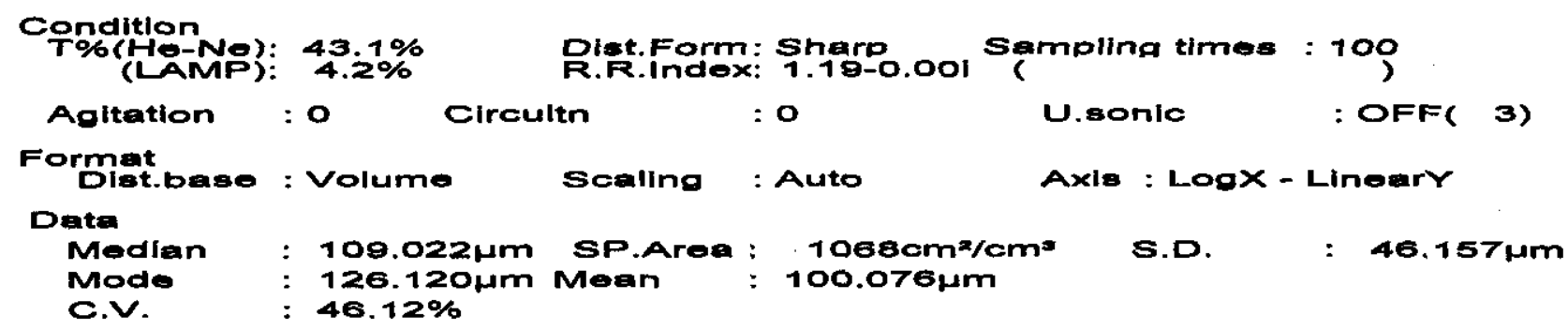

Span : (D 10.0-D 90.0)/D50 =1.170

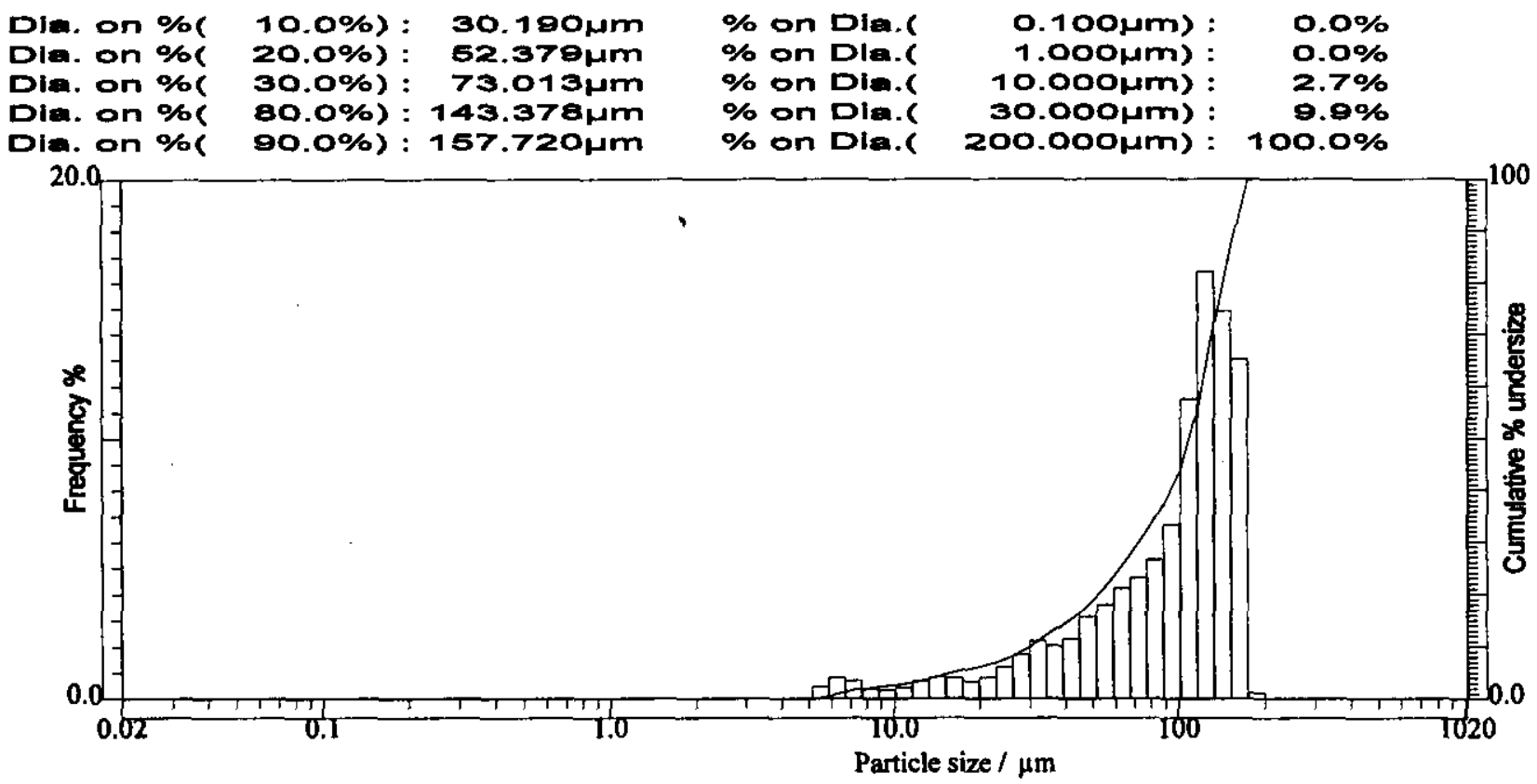

Sizo(1m) Froq(\%)Und(\%)

\begin{tabular}{lrl}
\hline 1019.5 & 0.00 & 100.00 \\
890.1 & 0.00 & 100.00 \\
777.1 & 0.00 & 100.00 \\
678.5 & 0.00 & 100.00 \\
592.4 & 0.00 & 100.00 \\
617.2 & 0.00 & 100.00 \\
451.6 & 0.00 & 100.00 \\
394.2 & 0.00 & 100.00 \\
344.2 & 0.00 & 100.00 \\
300.5 & 0.00 & 100.00 \\
282.4 & 0.00 & 100.00 \\
229.1 & 0.00 & 100.00 \\
200.0 & 0.23 & 100.00 \\
174.6 & 13.03 & 99.77 \\
152.5 & 14.91 & 86.74 \\
133.1 & 16.41 & 71.83 \\
116.2 & 11.53 & 55.42 \\
101.5 & 6.65 & 43.90 \\
88.58 & 5.29 & 37.24 \\
77.34 & 4.61 & 31.95 \\
87.52 & 4.21 & 27.35 \\
58.95 & 3.60 & 23.14 \\
51.47 & 3.15 & 18.54 \\
44.94 & 2.31 & 16.39 \\
39.23 & 2.02 & 14.07 \\
34.25 & 2.21 & 12.05 \\
29.91 & 1.69 & 9.85
\end{tabular}

Sizo(um) Freq(\%)Und(\%)

\begin{tabular}{lll}
\hline 26.11 & 1.23 & 8.16 \\
22.80 & 0.81 & 8.93 \\
19.90 & 0.86 & 6.11 \\
17.38 & 0.81 & 5.45 \\
15.17 & 0.83 & 4.85 \\
13.25 & 0.68 & 3.82 \\
11.56 & 0.44 & 3.14 \\
10.10 & 0.33 & 2.69 \\
8.816 & 0.39 & 2.36 \\
7.697 & 0.70 & 1.97 \\
6.720 & 0.81 & 1.28 \\
5.887 & 0.47 & 0.47 \\
5.122 & 0.00 & 0.00 \\
4.472 & 0.00 & 0.00 \\
3.905 & 0.00 & 0.00 \\
3.409 & 0.00 & 0.00 \\
2.978 & 0.00 & 0.00 \\
2.599 & 0.00 & 0.00 \\
2.269 & 0.00 & 0.00 \\
1.981 & 0.00 & 0.00 \\
1.729 & 0.00 & 0.00 \\
1.510 & 0.00 & 0.00 \\
1.318 & 0.00 & 0.00 \\
1.151 & 0.00 & 0.00 \\
1.005 & 0.00 & 0.00 \\
0.877 & $B .9 .00$ & 0.00 \\
0.786 & 0.00 & 0.00 \\
& &
\end{tabular}

Size(1,m) Freq(\%)Und(\%)

\begin{tabular}{lll}
\hline 0.889 & 0.00 & 0.00 \\
0.584 & 0.00 & 0.00 \\
0.510 & 0.00 & 0.00 \\
0.445 & 0.00 & 0.00 \\
0.389 & 0.00 & 0.00 \\
0.339 & 0.00 & 0.00 \\
0.298 & 0.00 & 0.00 \\
0.259 & 0.00 & 0.00 \\
0.228 & 0.00 & 0.00 \\
0.197 & 0.00 & 0.00 \\
0.172 & 0.00 & 0.00 \\
0.150 & 0.00 & 0.00 \\
0.131 & 0.00 & 0.00 \\
0.115 & 0.00 & 0.00 \\
0.100 & 0.00 & 0.00 \\
0.087 & 0.00 & 0.00 \\
0.076 & 0.00 & 0.00 \\
0.067 & 0.00 & 0.00 \\
0.058 & 0.00 & 0.00 \\
0.051 & 0.00 & 0.00 \\
0.044 & 0.00 & 0.00 \\
0.039 & 0.00 & 0.00 \\
0.034 & 0.00 & 0.00 \\
0.029 & 0.00 & 0.00 \\
0.026 & 0.00 & 0.00 \\
0.022 & 0.00 & 0.00 \\
& &
\end{tabular}




\section{DISTRIBUTION SHEET}

\begin{tabular}{|l|l|}
\hline To & From \\
\hline
\end{tabular}

Distribution

Project Title/Work Order

Results of Retrieval Studies with waste from Tank 241-C-104

Name

Fluor Hanford

Herting, D. L.

O'Rourke, J. F. (3)

Numatec Hanford Corporation

J. S. Garfield

J. R. Jewett

R. A. Kirkbride

L. L. Lockrem

W. I. Winters

CH2M HILL Hanford Group, Inc.

J. H. Baldwin

T. W. Crawford

S. G. Mckinney

R. W. Powell

Los Alamos Technical Associates, Inc.

E. J. Farris
From
TOPS

Page 1 of 1

Date February 3, 2000

EDT No. 620367

ECN No.

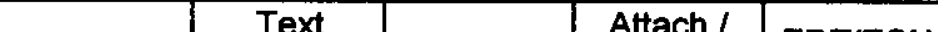

Attach./. EDT/ECN Attach.

T6-07

T6-07

$\mathrm{X}$

X

R3-73

R3-73

R3-73

T6-07

T6-07

$\mathrm{X}$

R2-12

$\mathrm{X}$
Only 